by

\begin{abstract}
Ali Nouhi
MSc, Mechanical Engineering, Shiraz University, Iran, 2011

BSc, Mechanical Engineering, Shiraz University, Iran, 2008
\end{abstract}

\author{
A dissertation \\ presented to Ryerson University \\ in partial fulfillment of the \\ requirement for the degree of \\ Doctor of Philosophy \\ in the program of \\ Mechanical and Industrial Engineering
}

Toronto, Ontario, Canada, 2017

(C) Ali Nouhi 2017 


\section{AUTHOR'S DECLARATION FOR ELECTRONIC SUBMISSION OF DISSERTATION}

I hereby declare that I am the sole author of this dissertation. This is a true copy of the dissertation, including any required final revisions, as accepted by my examiners.

I authorize Ryerson University to lend this dissertation to other institutions or individuals for the purpose of scholarly research.

I further authorize Ryerson University to reproduce this dissertation by photocopying or by other means, in total or in part, at the request of other institutions or individuals for the purpose of scholarly research.

I understand that my dissertation may be made electronically available to the public. 


\section{This is a manuscript-based dissertation on the basis of following publications:}

1) A. Nouhi, M.R. Sookhak Lari, J.K. Spelt, M. Papini, "Implementation of a shadow mask for direct writing in abrasive jet micro-machining", Journal of materials processing technology, vol. 223, pp. 232-239, 2015. (The first two authors contributed equally to this paper).

2) A. Nouhi, K. Kowsari, J.K. Spelt, M. Papini, "Abrasive jet machining of channels on highly-curved glass and PMMA surfaces”, Wear, vol. 356-357, pp. 30-39, 2016.

3) K. Kowsari, A. Nouhi, V. Hadavi, J.K. Spelt, M. Papini, "Prediction of the erosive footprint in the abrasive jet micro-machining of flat and curved glass", Tribology international, vol. 106, pp. 101-108, 2017. (Nouhi conducted the AJM and shadowgraphy experiments and carried out the experimental measurements and mathematical calculations. Kowsari led the development of the CFD simulations, and led the writing of the paper. Hadavi helped analyze the shadowgraphy results).

4) A. Nouhi, J.K. Spelt, M. Papini, "Abrasive jet turning of glass and PMMA rods and the micro-machining of helical channels", submitted to the Journal of Micromechanics and Microengineering 


\title{
ABRASIVE AIR JET MICRO-MACHINING OF HIGHLY CURVED SURFACES
}

\author{
Doctor of Philosophy, 2017
}

Ali Nouhi

Mechanical Engineering, Ryerson University

\begin{abstract}
\end{abstract}
In the abrasive jet micro-machining (AJM) process, a jet of high-speed particles is directed through a micro-nozzle which is used to erode a wide variety of materials. The micro-machining of small curved devices made of brittle and ductile materials is required in optical and biomedical equipment. This dissertation aims at fabricating axial grooves and helical micro-channels in stationary and rotating curved targets, respectively, using AJM. In addition, a model is proposed to predict the shape of machined channel profiles in glass and PMMA rods.

Since the air driven jet is divergent, the edges of the desired features are usually defined using a mask which is attached to the surface of the target material. This thesis presents an alternate technique using shadow masks that can be moved over the surface. It is demonstrated that this apparatus can be used to direct write features on the surface.

This dissertation proposes a modification to the existing surface evolution models for predicting the channel profiles machined on highly curved and tilted surfaces. It is shown that considering the change in local nozzle standoff and the divergence angle of each particle trajectory in the jet plume results in more accurate predictions.

Computational fluid dynamics (CFD) modeling showed that the jet footprint difference on the flat and curved surfaces was not due solely to the expected conical divergence in the jet, but also due to differences in the erosion caused by secondary impacts of rebounding particles. This observation has important implications for the surface profile modeling of curved surfaces.

Finally, a model for the prediction of the volumetric removal of material during the machining of rotating and translating PMMA and glass rods is presented. In addition, a new experimental procedure is proposed for machining helical micro-channels in glass and PMMA rods using a cylindrical steel spring as a mask. This method of machining provides a convenient means of fabricating helical micro-channels with different aspect ratios and radii of curvature. 
means of fabricating helical micro-channels with different aspect ratios and radii of curvature. Such helical micro-channels may have applications in inertial microfluidic devices where they can be used to aid liquid mixing and the separation of particles from a flow. 


\section{Acknowledgements}

I would like to thank:

$>$ Dr. Marcello Papini and Dr. Jan K. Spelt for providing me with the opportunity of joining their research team and their persistent and friendly support, encouragement, patience and advices in all steps of my PhD.

$>$ The financial support of the Natural Sciences and Engineering Research Council of Canada (NSERC) and the Canada Research Chairs Program.

$>$ Ryerson University for the fantastic facilities and equipment provided for this research.

$>$ Devin Ostrom, Chao Ma, Grace He, Joseph Amankrah, Alan Machin and Qiang Li, for their technical support. 


\section{Dedication}

This dissertation is dedicated to my beloved parents, Mahboobeh and Kazem, and brother, Mehdi, for their kindness and devotion, and for their endless support,

And

Niloofar without whom this thesis would have been completed one semester earlier. 


\section{Table of contents}

Author's declaration

Abstract $\quad$ iv

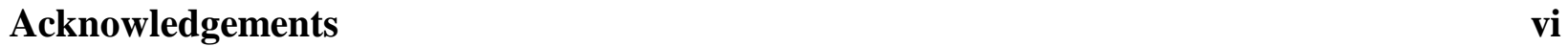

Dedication $\quad$ vii

List of tables

List of figures

$\begin{array}{ll}\text { Nomenclature } & \text { xviii }\end{array}$

$\begin{array}{ll}\text { Chapter } 1 \text { Introduction } & 1\end{array}$

1.1 Background and motivation 1

1.2 Literature review 2

1.2.1 Solid particle erosion mechanisms $\quad 2$

1.2.2 Modeling of feature shapes resulting from AJM $\quad 6$

1.2.3 Micro-machining of highly curved targets 12

$\begin{array}{lll}1.3 & \text { Objectives } & 13\end{array}$

Chapter 2 Implementation of a shadow mask for direct writing in abrasive jet micromachining

2.1 Introduction 14

2.2 Experiments 15

2.2.1 Shadow mask apparatus $\quad 15$

2.2.2 Measurement of channel cross-section and frosted region 18

2.3 Results and discussion $\quad 24$

2.3.1 Clamped vs. shadow mask 24

2.3.2 Effect of nozzle standoff distance $\quad 25$

2.3.3 Effect of shadow mask thickness 27

$\begin{array}{ll}2.3 .4 \text { Mask standoff } & 29\end{array}$

2.3.5 Effect of particle size $\quad 31$

2.3.6 Crossed shadow masks

$\begin{array}{ll}2.4 \text { Summary } & 35\end{array}$

Chapter 3 Abrasive jet machining of channels on highly-curved glass and PMMA surfaces

3.1 Introduction $\quad 37$

3.2 Surface profile modelling 42 
3.2.1 Background

3.2.2 Generalized surface evolution model - varying standoff distance and jet divergence angle

3.2.3 Development of generalized model- brittle materials 46

3.2.4 Ductile materials $\quad 49$

3.2.5 Determination of erosive efficacy 49

3.2.6 Initial conditions $\quad 52$

3.3 Experiments $\quad 53$

3.3.1 Apparatus $\quad 53$

3.3.2 Channels on flat surfaces

3.3.3 Channels in rods 54

3.4 Results and discussion $\quad 55$

3.4.1 Erosive efficacy function 55

3.4.2 Prediction of channel profiles using new model $\quad 56$

$\begin{array}{ll}\text { 3.4.2.1 Channels in tilted flat glass target } & 56\end{array}$

$\begin{array}{lll}\text { 3.4.2.2 Channels in tilted flat PMMA target } & 58\end{array}$

$\begin{array}{lll}\text { 3.4.2.3 Channels in glass rods } & 59\end{array}$

3.4.2.4 Channels in PMMA rod 64

3.4.3 Predicted channel profiles using previous models 65

$\begin{array}{ll}3.5 \text { Summary } & 67\end{array}$

Chapter 4 Prediction of the erosive footprint in the abrasive jet micro-machining of flat and curved glass

$\begin{array}{ll}4.1 \text { Introduction } & 69\end{array}$

$\begin{array}{ll}4.2 \text { Experiments } & 70\end{array}$

4.2.1 Jet and footprint measurements $\quad 71$

4.2.2 CFD modeling $\quad 72$

$\begin{array}{lll}4.3 & \text { Results and discussion } & 75\end{array}$

$\begin{array}{ll}\text { 4.3.1 AJM jet structure } & 75\end{array}$

4.3.1 Erosive footprint prediction for flat surfaces $\quad 80$

$\begin{array}{lll}\text { 4.3.1.1 Experimental validation } & 86\end{array}$

4.3.2 Erosive footprint prediction for curved surfaces $\quad 88$

4.4 Implications for AJM 93

$\begin{array}{ll}4.5 \text { Summary } & 94\end{array}$

Chapter 5 Abrasive jet turning of glass and PMMA rods and the micro-machining of $\begin{array}{ll}\text { helical channels } & 96\end{array}$

5.1 Introduction: $\quad 96$

5.2 Experiments 99

5.2.1 AJM apparatus $\quad 99$

$\begin{array}{lr}\text { 5.2.2 AJM lathe } & 99\end{array}$

5.2.3 Unmasked lathe machining 101 
5.2.4 Spring-masked helical channels on rods 102

5.3 Modelling 104

5.3.1 Prediction of surface evolution for masked helical channels 104

5.3.2 Prediction of volume of removed material in unmasked AJM lathe operations 106

$\begin{array}{ll}5.4 \text { Results and discussion } & 112\end{array}$

5. 4.1 Unmasked AJM lathe operations 112

5.4.1.1 Volume of removed material 112

5.4.1.2 Effect of nozzle offset 114

5.4.2 Masked helical channels 116

5.4.2.1 Surface evolution of helical channel profiles 116

5.4.2.2 Effect of mask spring wire diameter (mask thickness) on dimensions of helical channels 127

5.4.2.3 Effect of spring pitch on the blast lag 129

$\begin{array}{ll}5.5 \text { Summary } & 130\end{array}$

$\begin{array}{ll}\text { 6.1 Summary } & 131\end{array}$

$\begin{array}{llr}\text { 6.2 Conclusions } & 133\end{array}$

$\begin{array}{ll}\text { 6.3 Contributions } & 135\end{array}$

$\begin{array}{ll}\text { 6.4 Recommendations for Future work } & 137\end{array}$

$\begin{array}{lr}\text { References } & 139\end{array}$ 


\section{List of tables}

Table 2.1 Summary of conditions used in each experiment. ............................................. 23

Table 5.1 Axial feed speeds used to obtain the specified helix angles for a constant rotational speed of $57 \mathrm{rpm}$ in the AJM lathe experiments.

102 


\section{List of figures}

Fig. 2.1 Shadow mask apparatus. Nozzle standoff $(N S)$ and mask standoff $(M S)$ were defined relative to the top of the mask. Only two of three springs shown. ......................................... 17

Fig. 2.2 Relative orientation of nozzle and shadow mask. 17

Fig. 2.3 Jet aperture for direct writing of curved paths made using two sets of crossed shadow masks 17

Fig. 2.4 Location of edge of frosted area (dashed line) and a comparison of channel width based on (i) the slope method (dashed dotted line) and (ii) the channel crater size method (dotted line).

Fig. 2.5 Top view of glass channel blasted at $200 \mathrm{kPa}, 20 \mathrm{~mm}$ nozzle standoff, with a contacting crossed shadow mask having a square opening $260 \mu \mathrm{m}$ on a side. The $30 \mu \mathrm{m}$ wide frosted regions

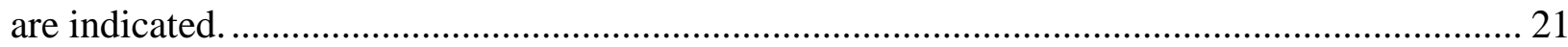

Fig. 2.6 Segmented image of Fig. 2.6 showing the frosted regions in white............................. 22 Fig. 2.7 Cross-sectional profiles of channels machined using various number of passes and the shadow mask of Fig. 2.3 in contact with the surface (solid and dashed lines) or a clamped mask (symbols). Dimensions normalized using the mask opening, $W=293 \mu \mathrm{m}$. Only half of each symmetric profile is shown. Mask thickness of $800 \mu \mathrm{m}, N S=20 \mathrm{~mm}$. 25

Fig. 2.8 Comparison of typical channel profiles obtained after 4 passes of the shadow mask of Fig. 2.3 at three nozzle standoff distances (Fig. 2.2). Depth and width normalized by mask opening $W=270 \mu \mathrm{m}$ 26

Fig. 2.9 Average channel depth (squares) and width (circles) at three nozzle standoffs. Scatter bars representing \pm 1 standard deviation for 9 measurements ( 3 measurements on each of 3 specimens) were small enough to fit within the symbols. 26

Fig. 2.10 Average frosted width at three nozzle standoffs (Fig. 1). The scatter bars represent \pm 1 standard deviation for 9 measurements ( 3 measurements on each of 3 specimens). 27

Fig. 2.11 Effect of mask thickness on typical channel profiles obtained after 4 passes of the shadow mask of Fig. 2.3. Channel depth and width normalized by mask opening $W=270 \mu \mathrm{m} . \ldots \ldots \ldots \ldots \ldots . . . .28$ Fig. 2.12 Average channel depth, width and frosted width for mask thicknesses of 0.8 and $1.6 \mathrm{~mm}$. The scatter bars represent \pm 1 standard deviation for 9 measurements ( 3 measurements on each of 3 specimens) 28 
Fig. 2.13 A particle striking the edge of a thin mask (a) is more likely to strike the surface, while that striking a thick mask (b) collides with the opposing mask ............................................. 29 Fig. 2.14 Typical channel profiles obtained after 4 passes of the shadow mask of Fig. 2.3 using mask-to-surface standoff distances of 0 and $2 \mathrm{~mm}$ (Fig. 1). Channel depth and width normalized by mask opening $W=270 \mu \mathrm{m}$. . . . 30

Fig. 2.15 Average depth, width and frosted width for mask standoffs of 0 and $2 \mathrm{~mm}$. The scatter bars represent \pm 1 standard deviation for 9 measurements ( 3 measurements on each of 3 specimens).

Fig. 2.16 Channel profiles obtained after 4 passes of the shadow mask of Fig. 2.3 using 10 and 25 $\mu \mathrm{m}$ particles. Channel depth and width normalized by mask opening $W=290 \mu \mathrm{m}$. 32 Fig. 2.17 Average channel depth, width and frosted width for particle sizes of 10 and $25 \mu \mathrm{m}$. The scatter bars represent \pm 1 standard deviation for 9 measurements ( 3 measurements on each of 3 specimens)...

Fig. 2.18 Profiles of channels made after 4 passes of the crossed shadow mask (Fig. 2.4) and the regular shadow mask (Fig. 2.3). Channel depth and width normalized by mask opening $W=290$ $\mu \mathrm{m}$.

Fig. 2.19 Footprints of the erodent jets produced using the crossed shadow mask (left) and the regular mask (right). For the crossed mask, the slot of the top set of masks was in the vertical direction. 35

Fig. 2.20 Ryerson University logo machined in glass using direct writing technique with crossed shadow masks. 35

Fig. 3.1 Schematic of channel machining processes. (a) Channel made at normal impact angle on flat target, (b) Channel made at oblique impact angle on flat target, (c) Channel made axially on rod. Conical divergence of particle jet causes local standoff and impact angle to vary across footprint in parts (b) and (c).

Fig. 3.2 centerline (e.g. $V_{l}$ and $V_{2}$ ) and coordinate system is located on the target, (b) new model where incident angle varies across jet and coordinate system is located at nozzle tip. $n_{1}$ and $n_{2}$ are unit vectors normal to channel profile at point of impact, $\psi_{1}$ and $\psi_{2}$ define local angle between impact velocity vector and outward normal to surface. $h_{0}$ is the nominal standoff distance..... 45 
Fig. 3.3 Particle impact on rod at jet centerline $(f)$ and at edge of nozzle footprint $(g)$ illustrating variation in local impact angle, $\alpha$, and standoff due to target curvature and jet divergence. ....... 46 Fig. 3.4 Schematic representation of intersection of abrasive jet plume with successive planes at standoffs of $10 \mathrm{~mm}, 20 \mathrm{~mm}$ and $30 \mathrm{~mm}$, where shallow channels were machined to determine the erosive efficacy function........................................................................................................ 51

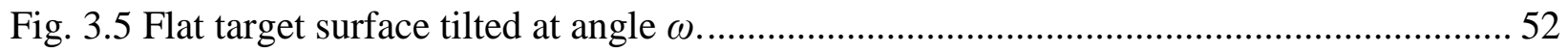

Fig. 3.6 Measured profiles (symbols) and curve fits (dashed lines) of three shallow channels machined at $10 \mathrm{~mm}, 15 \mathrm{~mm}$ and $20 \mathrm{~mm}$ nozzle standoffs on a flat glass surface...................... 56 Fig. 3.7 Measured (symbols) and predicted (dashed lines) profiles of channels machined on glass at a nozzle angle of $30^{\circ}$. Predictions used the new model, Eq. (13), with the three-dimensional erosive efficacy, Eq. (16) (Section 2.3). Arrows show direction of nozzle inclination. Note difference in depth and width scales. 57

Fig. 3.8 Measured (symbols) and predicted (dashed lines) profiles of channels machined in PMMA at a nozzle angle of $30^{\circ}$. Predictions used the new model, Eq. (15), with the three-dimensional erosive efficacy, Eq. (16) (Section 2.3). Arrows show direction of nozzle inclination. Note difference in width and depth scales. 58

Fig. 3.9 CFD results illustrating the effect of particle second strike on (a) flat target (b) $5 \mathrm{~mm}$ and (c) $3 \mathrm{~mm}$ glass rods. The distance from inlet to target was $10 \mathrm{~mm}$ in all cases. 61 Fig. 3.10 Measured cross-sectional profiles (symbols) of channels machined axially along glass rods of diameter: (a) $3 \mathrm{~mm}$, and (b) $5 \mathrm{~mm}$ compared with predictions (solid lines) of Eq. (13) using the three-dimensional erosive efficacy where $\beta=20$ and 22 for $3 \mathrm{~mm}$ and $5 \mathrm{~mm}$ rod, respectively, Eq. (16). 63

Fig. 3.11 Measured cross-sectional profiles (symbols) of channels machined axially in $4.72 \mathrm{~mm}$ diameter PMMA rod compared with predictions (solid lines) of Eq. (15) using the threedimensional erosive efficacy where $\beta=22$, Eq. (16).

Fig. 3.12 Comparison of measured asymmetric channel cross-sections in a flat glass plate with predictions of present model (Eq. (13)) and that of ref. [28]. Arrows show direction of incident particles at a jet angle of $30^{\circ}$. 66

Fig. 3.13 Comparison of measured channel cross-sections in a $5 \mathrm{~mm}$ diameter glass rod with predictions of present model (Eq. (13)) and model of ref. [11]. 67 
Fig. 4.1 Double-pulsed shadowgraphy apparatus. 72

Fig. 4.2 Domains and boundary conditions of: (a) 2D axisymmetric CFD model of the impingement of an air-particle jet on a flat target and (b) 3D CFD model of the impingement of an air-particle jet on a curved target. 75 Fig. 4.3 AJM jet. (a) Microscope images of the AJM jet. (b) Air and particle velocity magnitude contours obtained using CFD. 78

Fig. 4.4 Radial distribution of particles within the jet: (a) at nozzle exit (b) at $20 \mathrm{~mm}$ standoff, obtained from shadowgraphy. The error bars indicate the standard deviations obtained from three measurements of approximately 15000 particles each. 79

Fig. 4.5 Impingement of AJM jets on flat targets. Air velocity magnitude contours and $10 \mu \mathrm{m}$ diameter particle trajectories for standoff distances of: (a) $5 \mathrm{~mm}$, (b) $10 \mathrm{~mm}$, (c) $20 \mathrm{~mm}$, and (d) $30 \mathrm{~mm}$. (e) Drag energy loss as a function of standoff distance for particles released at the nozzle centerline obtained from either $h_{\text {apex }}$ and $V_{r}$ or direct integration of $F_{d}$ (Eq. (2)). (f) CFD prediction of particle drag force versus particle rebound displacement for different standoffs. (g) Axial velocities of particles released from a given mesh element at the inlet boundary approximately 100 $\mu \mathrm{m}$ from the nozzle centerline (13\% of the nozzle diameter) at various distances from the target using the models of Fig. 4.5. 86 Fig. 4.6 (a) Predicted (dashed lines) and measured (solid lines) erosive footprint diameter versus standoff with and without secondary particle impacts. The lines are to guide the eye only. Error bars represent \pm 1 standard deviation for 3 measurements. (b) Schematic representation of intersections of primary and secondary plumes with successive target planes at standoffs of $10 \mathrm{~mm}$ and $30 \mathrm{~mm}$. $\psi$ defines the second-strike cone angle, and $h^{\prime}$ is the apex height of a corresponding

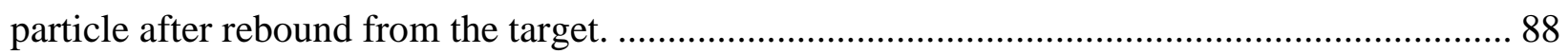
Fig. 4.7 Impingement of AJM jets on curved targets at a standoff of $20 \mathrm{~mm}$. Air velocity magnitude contours and particle trajectories for rod diameters of: (a) $5 \mathrm{~mm}$, and (b) $3 \mathrm{~mm}$. 90 Fig. 4.8 CFD-obtained normalized erosion maps on a flat target and a $5 \mathrm{~mm}$ diameter rod. Each map was normalized by its maximum specific erosion rate (mass eroded per unit mass of erodent).

Fig. 4.9 A schematic of Weibull-type function describing the shallow eroded profile. The coordinates $(\mathrm{y}, \mathrm{x})$ of a typical point on the profile are shown. 92 
Fig. 4.10 Normalized erosive efficacies and the best fits (Weibull distribution) for flat and curved ( $5 \mathrm{~mm}$ diameter) glass targets. The abscissa was normalized by standoff distance and the ordinate was normalized by the depth of the channel centerline.

Fig. 5.1 a) AJM lathe, b) a schematic of a helical channel machined in one nozzle pass.

Fig.5.2 Definition of helix pitch, $c$, helix angle, $\psi$, radius at the rod surface, $r$, and angular position, $\theta$. The coordinate system is shown with an offset from its actual origin, point $O$, for clarity.... 108 Fig.5.3 Calculated erosive efficacy over one footprint 110 Fig.5.4 Schematic of the process of superposition of erosive efficacy to obtain the resultant profile for unmasked lathe machining of rods. Cross-sectional view of half the rod 111 Fig. 5.5 Measured and predicted AJM lathe machining rate for: a) $5 \mathrm{~mm}$ glass rod, b) $4.72 \mathrm{~mm}$ PMMA rod. Predictions used the model of Section 3.2, c) measured cross sectional profile and model prediction for $5 \mathrm{~mm}$ diameter glass rod. The experiments were repeated twice and measurements were performed three times (i.e., the data points represent the average of 6 measurements). 114 Fig. 5.6 Schematic of nozzle offset $\Delta$, with $\Upsilon_{1}$ and $\Upsilon_{2}$ the particle impact angles to the surface of the rod at the boundaries of the jet plume 115 Fig. 5.7 Measured (symbols) and predicted (dashed lines) cross-sectional profiles of masked helical channels machined in a) rotating $5 \mathrm{~mm}$ glass rod, spring pitch $900 \mu \mathrm{m}$ (low aspect ratio), b) rotating $5 \mathrm{~mm}$ glass rod, spring pitch $700 \mu \mathrm{m}$ (high aspect ratio) c) rotating $4.72 \mathrm{~mm}$ PMMA rod, spring pitch $1200 \mu \mathrm{m}$ (low aspect ratio), d) rotating $4.72 \mathrm{~mm}$ PMMA rod, spring pitch 600 $\mu \mathrm{m}$ (high aspect ratio). Predictions used the model of Eq. (5). A dashed outline of spring (mask) location is shown in all the images. 122 Fig. 5.8 Optical microscope images of helical microchannels machined in: (a) $2 \mathrm{~mm}$ glass rod (aspect ratio 0.7, $500 \mu \mathrm{m}$ wire diameter, spring pitch $900 \mu \mathrm{m}$ ), (b) $3 \mathrm{~mm}$ glass rod (aspect ratio 1.2, $400 \mu \mathrm{m}$ wire diameter, spring pitch $800 \mu \mathrm{m}$ ), (c) $5 \mathrm{~mm}$ glass rod (aspect ratio 1, $700 \mu \mathrm{m}$ wire diameter, spring pitch $750 \mu \mathrm{m}$ ), and (d) $4.72 \mathrm{~mm}$ (aspect ratio 0.6, $400 \mu \mathrm{m}$ wire diameter, spring pitch $800 \mu \mathrm{m}$ ) PMMA rod. A dashed outline of spring (mask) location is shown in all the images. 
Fig. 5.9 Particles striking the wire of a thick spring (a) were more likely to ricochet and strike the opposite coil, while those striking a thinner spring (b) had a higher probability of rebounding

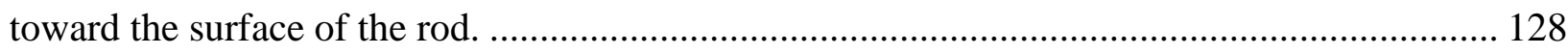

Fig. 5.10 Channel profiles machined on $5 \mathrm{~mm}$ diameter glass rod using a thin, $400 \mu \mathrm{m}$, (symbols) and a thick, $750 \mu \mathrm{m}$, (dashed lines) spring mask (The experimental conditions were as in Section 2.4)

Fig. 5.11 The blast lag in a $5 \mathrm{~mm}$ glass rod illustrated for two channel widths. Wider channels made with a larger pitch $(650 \mu \mathrm{m}$ in (b)), became deeper for a given particle dose than narrower ones made using a smaller pitch, $(300 \mu \mathrm{m}$ in (a)) 129 


\section{Nomenclature}

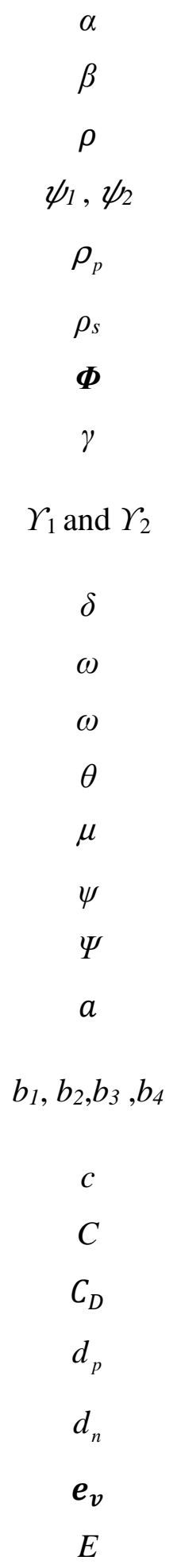

Impact angle

Focus coefficient

Density of the particle

Local angle between impact velocity vector and outward normal to surface

Particle density in Eq. 4.1

Density of the target

Particle mass flux

Particle trajectory angle

Particle impact angles to the surface of the rod at the boundaries of the jet plume

Related to shallow channel profile depth

Arbitrary angle of inclination of the target

Angular speed in Eq. 5.12

Inclination angle of nozzle

Dynamic viscosity of air

Second-strike cone angle

Helix angle in Eq. 5.12

Depth of a hemispherical crater

Functions of the ratio of the surface area of an equivalent sphere to the actual particle surface area

Pitch of the spring divided by $2 \pi$ in Eq. 5.11

Erosion constant

Drag coefficient

Particle diameter in Eq. 4.1

Nozzle diameter

Particle velocity unit vector

Erosion 


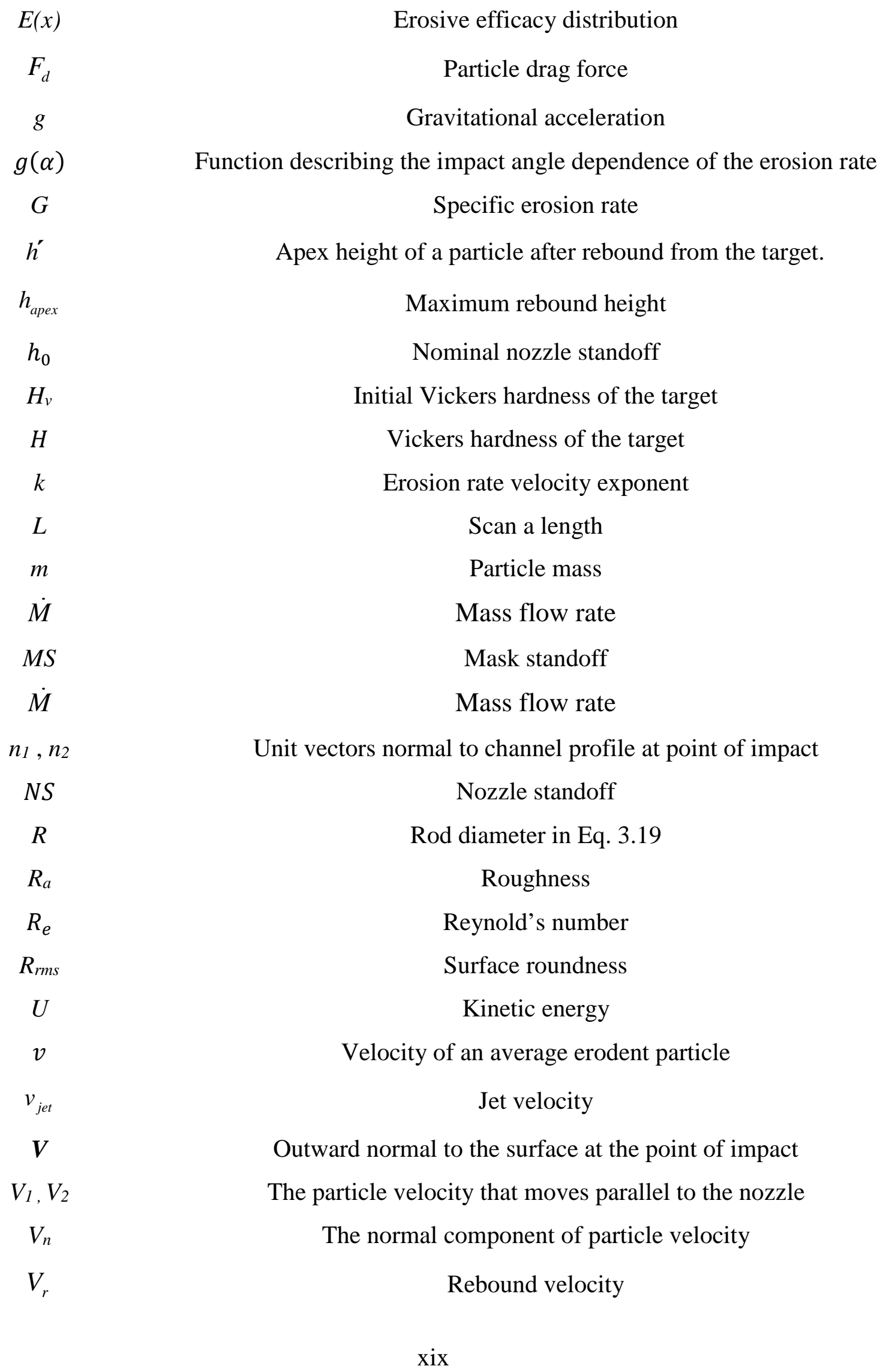




$\begin{array}{cc}V_{f}, & \text { Axial feed speed } \\ y & \text { Transverse coordinate along the channel width in Eq. } 4.4 \\ y^{+} & \text {Dimensionless wall coordinate } \\ Z & \text { Profile depth } \\ Z, t & \text { Partial derivatives of the profile depth with respect to time } \\ Z, x & \text { Partial derivatives of the profile depth with respect to } x \\ \theta & \text { Angular coordinate in Eq. } 5.11\end{array}$




\section{Chapter 1 Introduction}

\subsection{Background and motivation}

Abrasive jet micro-machining (AJM) uses a jet of abrasive particles to etch micro-features and has the ability to cut and mill many materials without excessive forces or thermal damage, which is a significant advantage over many other machining technologies [1]. The micromachining of small curved devices made of brittle materials, such as glass and ceramics, and ductile materials like polymers and metals has wide applications in optical, biomedical and microfluidic devices [1]. A typical AJM setup consists of following: a Micro-Abrasive Blaster, operating at $200-500 \mathrm{kPa}$ air pressure that passes through a 0.46-1.5 mm diameter nozzle; a dehumidifier, a refrigeration air dryer, and a desiccant filter on the inlet air that minimizes the moisture in the powder reservoir; a variable-speed rotary electric mixer to avoid powder bed compaction and particle agglomeration inside the reservoir (Fig. 1.1). 10-50 $\mu \mathrm{m}$ granular particles (usually $\mathrm{Al}_{2} \mathrm{O}_{3}$ ) are used as the abrasive for machining. The effective process parameters that define the etch rate are air pressure, nozzle shape, size, standoff distance and inclination.

AJM has a largely unexplored potential for machining tilted flat and stationary and rotating curved surfaces, such as found in the turning of small cylindrical parts, the machining of microfeatures on rods, and making threads on glass and ceramics which are otherwise difficult to machine [1]. Consequently, the AJM of highly-curved surfaces has not received notable attention. A functional theoretical model that could be implemented to predict the shape of channel profiles machined on curved targets has not been developed, although mathematical models have been provided for modelling grooves and holes profiles machined in flat substrates at normal incidence. The lack of such modelling and the challenges that it may have are the prime motivations for this work. Moreover, an inexpensive and convenient means of fabricating helical micro-channels on small sized highly curved glass and PMMA rods has potential application in microfluidic devices, e.g. flow mixing and particle separation from flow. 


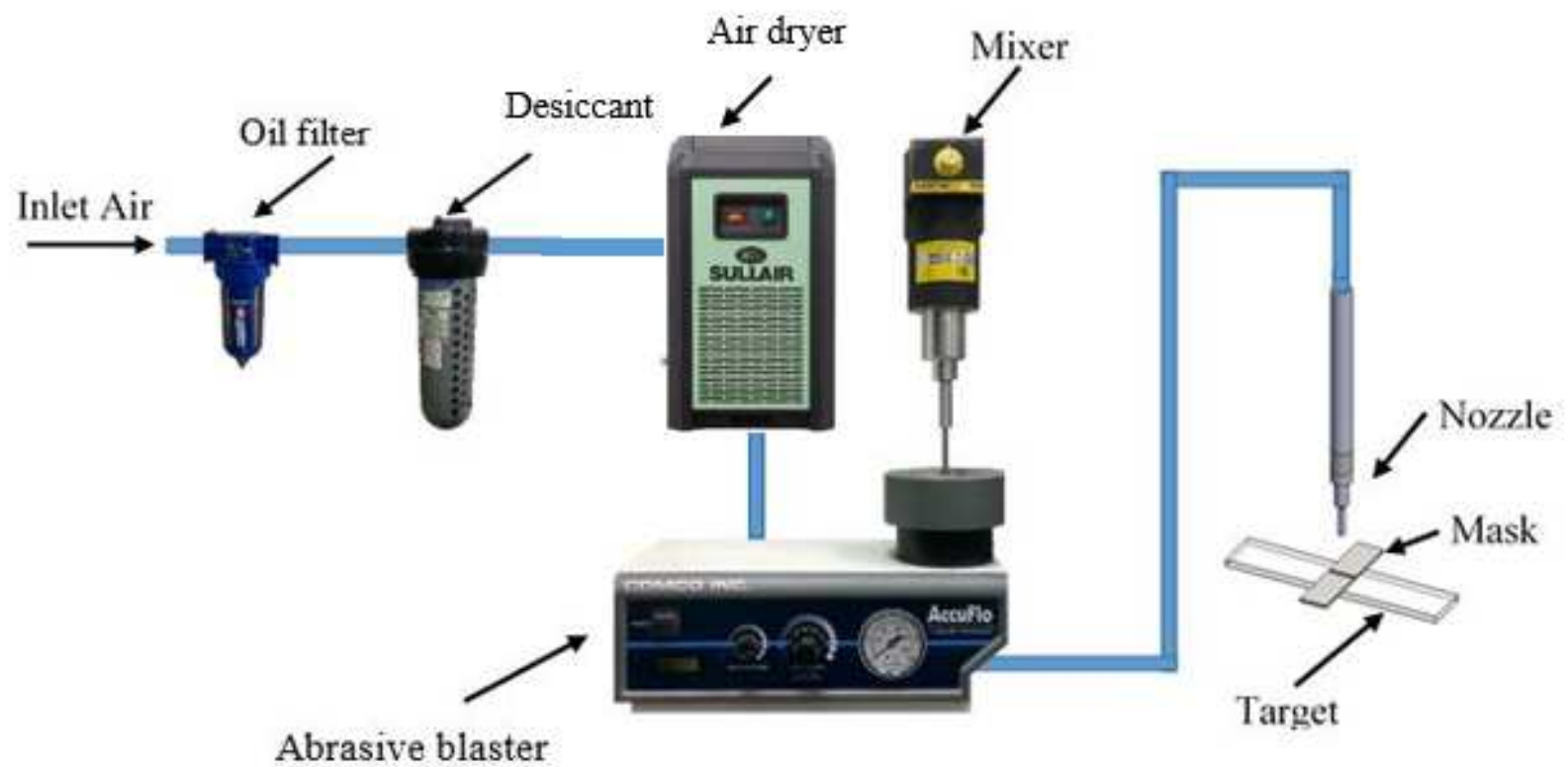

Fig. 1.1 Schematic of a typical of AJM apparatus.

\subsection{Literature review}

In this section, a brief literature review is presented to facilitate a concise overview of the previous investigations about solid particle erosion mechanisms and landmark work on AJM and its modelling. It is intended to illustrate the areas where further research is required. More extensive literature reviews on specific topics are provided at the beginning of Chapters 2-5.

\subsubsection{Solid particle erosion mechanisms}

Various opinions exist on the mechanisms of material removal when small high-speed particles strike a target, i.e., in the solid particle erosion process. The mechanism depends on several factors such as impact angle, particle shape and the type of material being impacted. The dependence of material removal mechanism on the angle of impact was known as early as 1946 [2]. Several mechanisms have been proposed and wear models for the purpose of estimating the amount of damage have been presented. Finnie [2] considered that a tip of a rigid abrasive cuts and removes material in ideal manner from a ductile metal. Sheldon and Finnie [3] proposed that for ideal brittle materials, material removal occurs by propagation and intersection of cracks. The 
above mentioned works clearly show that the material removal mechanism depends on the type of the material eroded. Nevertheless, some researchers have attempted to cover both brittle and ductile materials with the same wear model. For example, Bitter [4] and [5] proposed that erosion consists of two simultaneous processes, cutting wear and deformation wear, a theory viewed skeptically by Finnie [2]. According to Bitter, at high angles the dominant mechanism is deformation wear while at low angles cutting wear dominates.

The erosion rate and material removal rate vary for different impact angles, which fall in the practical range of $10-90^{\circ}$. Erosion mechanisms in AJM can be classified as either brittle or ductile. Brittle erosion exhibits deformation wear and fracture while ductile erosion exhibits cutting wear [5]. At oblique impact angles, ductile erosion is promoted, while at impact angles approaching normal incidence, brittle erosion is favored [5]. Brittle erosion occurs in hard substrates such as glass [6]. For example, for unmasked glass, the erosion rate is maximum at normal incidence $\left(\alpha=90^{\circ}\right)$, when the nozzle is perpendicular to the surface, and decreases for decreasing impact angles [7]. Ductile erosion occurs in soft substrates, such as poly methyl methacrylate, PMMA, (a polymer) [6], where the erosion rate increases with the impact angle up until a maximum value of about $15-40^{\circ}$, after which it begins to exponentially drop off [6],[7]. However, some polymers can exhibit intermediate/semi-brittle behavior [8]. For example, the maximum erosion rate in an E-glass/epoxy composite occurs at 45-60 [8]. Other polymers such as quartz-polyimide, glass-epoxy and quartz-polybutadiene composites exhibit brittle erosion [8].

The erosion rate for brittle materials can be expressed in terms of the incident particle velocity raised to the exponent $k$, which is a function of the hardness and toughness of the particles and target, as well as the particle size, type and velocity [9]. It is assumed that kinetic energy loss due to particle breakage and deformation results in $k<2$, [10]. For glass substrates with aluminum oxide particles, $k$ is in the range of 2-4 [9]. Hence, the erosion rate for a brittle erosive system can be defined as [9],

$$
E_{r}=C(V \sin \alpha)^{k}
$$

where $E_{r}$ is the volume or mass of target material removed per mass of erodent particles impacting, $V$ is the particle velocity, $\alpha$ is the angle the jet makes with the surface, $k$ is a velocity exponent and $C$ is an empirical constant which generally depends on particle and target hardness, as well as 
particle size, type. For the same substrate, the velocity exponent usually increases with larger particle size, lower toughness and hardness of particles, and with higher particle velocity, [9].

A standard method of obtaining $k$ for brittle materials requires performing erosion rate measurements at varying velocities, which have to be measured a priori for different process conditions [9]. A more reliable method of obtaining $k$ is by performing shallow erosion rate measurements for different impact angles and curve fitting a normalized erosion rate relationship (Eq. 1.1) (normalized with respect to erosion rate at normal impact), when plotted against the impact angle [9]. This eliminates the need for measuring the velocity. The normalized erosion rate $E_{r}^{*}$ can be expressed as [11]:

$$
E_{r}^{*}=(\sin \alpha)^{k}
$$

As it can be seen, the velocity vanishes from the normalized relationship.

The erosion rate for ductile materials is a more complex function of the impact angle obtained experimentally, and can be expressed as:

$$
E_{r}(\alpha)=g(\alpha) E_{r, 90}
$$

where $E_{r}(\alpha)$ and $E_{r, 90}$ are the erosion rate at an arbitrary impact angle, $\alpha$, and at normal incidence $\left(\alpha=90^{\circ}\right)$, respectively, and $g(\alpha)$ is a function expressing the impact angle dependency of the erosion rate [12]. $g(\alpha)$ is a semi-empirical quantity and is a product of two parameters, one characterizing the cyclic plastic deformation, and the other the cutting action [12],

$$
\mathrm{g}(\alpha)=(\sin \alpha)^{n_{1}}\left(1+H_{v}(1-\sin \alpha)\right)^{n_{2}}
$$

Equation (1.4) describes the dependence of the erosion rate on the impact angle, $\alpha$, defined between the incident velocity vector and the local tangent to the surface as: 


$$
\alpha=\frac{\pi}{2}-\arccos \left(\frac{1}{\sqrt{1+z, x^{2}}}\right)
$$

$H_{v}(\mathrm{GPa})$ is the initial target hardness and the constants $n_{1}$ and $n_{2}$ are determined experimentally and depend on the particle hardness and other impact conditions and $z_{x, x}$ is the partial derivative of the profile depth $z$ as a function of the coordinate $x$ [12].

Erosion as it applies to abrasive jet machining operations can be performed at either normal $\left(\alpha=90^{\circ}\right)$ or oblique $\left(\alpha<90^{\circ}\right)$ incidence of the jet, and either with or without a mask to define the eroded feature dimensions. For erosion at normal incidence, the mechanism is similar in masked and unmasked features except that in masked features, mask wear contributes to variations in erosion, in addition to erosion by primary particles and particles rebounding from the feature side walls as it becomes deeper (which gives it the parabolic shape) [7]. This side wall inclination can be reduced if smaller particles are used which can also lead to flatter bottoms [13]. For oblique erosion, similar conclusions are drawn with the exception that additional undercutting near one of the sides/mask edges is observed, due to over-etching caused by rebounding particles, in addition to over-etching caused by mask wear [7]. The total over-etching eventually subsides with increasing impact angle but increases with scanning times and feature width [7]. Moreover, in the case of tilted blasting, particle hindering occurs, i.e. one of the masks shadows a portion of the jet plume and thus decreases the particle dose delivered to a region of the target in its shadow.

Resulting shapes of masked and unmasked features machined in AJM are different for brittle and ductile erosive systems. For brittle erosion such as that which normally occurs in glass, unmasked channel profiles resemble a parabolic shape (with shallow sidewalls) and for masked channels and profiles the resulting features have higher aspect ratio (AR, i.e. ,depth to width ratio) with steeper sidewalls [9]. For ductile erosion, such as in PMMA, the resulting channel profiles have much steeper sidewalls and flatter central regions when a mask is used, when compared to AJM features machined in glass [6].

Most AJM applications are realized via the use of relatively thin, polymeric contact masks in the thickness range of 50-150 $\mu \mathrm{m}$. One example is the combination of two polymers: a brittle epoxy resin SU8, known for its photosensitivity, and the elastic and thermocurable polydimethylsiloxane (PDMS), known for its high erosion resistance [14]. Polymeric masks are usually spin-coated and post-baked at around $100^{\circ} \mathrm{C}$ [40]. Polymeric masks are more wear resistant 
than metallic masks and can achieve smaller feature sizes (as small as $30 \mu \mathrm{m}$ ) [15] but attain smaller aspect ratio features (up to about 1), and are more difficult to apply and remove. However, the use of a gold nanoparticle dispersed polyurethane (AuPU4) polymer mask can increase achievable ARs to about 2.1 [16]. Other masking materials include photosensitive elastomer foils such as Rayzist in the thickness range of 50-70 $\mu \mathrm{m}$. However, these masks exhibit poor adhesion to the substrate above particle velocities of $200 \mathrm{~m} / \mathrm{s}$ [17]. The other method is using self-adhesive ultraviolet (UV) light curing masks (e.g., RapidMask (RM), IKONICS Corp) which is utilized for machining channels and making patterns. Ahmadzadeh et al. [18], investigated RM for making microfluidic channels on flat targets. Ghobeity at al. [19] fabricated low-aspect-ratio microfluidic chips using these RMs. Thick steel masks are also used as an alternative masking technique since they can be easily patterned via laser cutting, and copper and zinc masks because of their wide use in electroplating and forming operations [9],[17]. Metal masks can be implemented to achieve high aspect ratios (up to about 3) [9]. Their use also minimizes mask undercutting that can result from curvature caused by the plastic deformation of the mask by the particle jet [9]. Copper masks have a higher erosion resistance than steel masks and can achieve feature sizes as small as $50 \mu \mathrm{m}$ [20]. These masks have a thickness range of $0.5-3 \mathrm{~mm}$.

\subsubsection{Modeling of feature shapes resulting from AJM}

Models for profile evolution in masked glass substrates were developed by ten Thije Boonkkamp-Jansen [21] and Slikkerveer et al. [22]. The models (described in more detail in Section 3.2.3) assumed that the resulting profile shape originated from the dependence of erosion rate on the impact angle and the velocity exponent, Fig. 1.2. In Ref. [22], the erosion rate was modeled in the form of erosion efficiency (similar to Eq. (1.1)), which is based on the stiffness, hardness and fracture toughness of the substrate [11], [23], where the influence of impacting particles was described by their kinetic energy raised to the power of the velocity exponent. The local erosion velocity of the surface was obtained by multiplying the erosion efficiency by the energy flux of the particle kinetic energy and dividing it by the substrate density. The authors of Ref. [21] related the developing surface slope to local erosion via the normal component of velocity that approximately (i.e., linearly) accounted for side wall inclination caused by particle collisions with the mask edge. For both models [21] and [22], only the normal component of velocity to the 
surface was assumed to contribute to the erosion process. The models resulted in profiles with sharp cusps at the center that over-predicted the profile depth (in comparison to experimental profiles) due to their simplicity and because they did not consider the decrease in erosion rate with time and hence the effect of surface curvature.

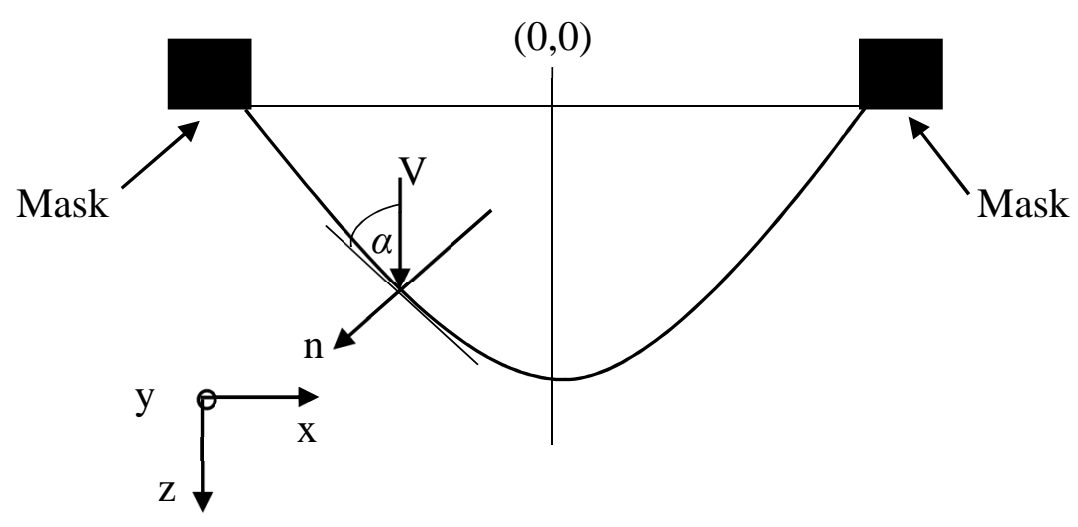

Fig. 1.2 Schematic of a channel cross sectional profile, demonstrating unit normal to the surface, $n$, impact angle, $\alpha$, particle velocity, $V$ and coordinate system.

The model of Ref. [22] was extended to include the 'second strike' effect where particles that originally impacted the surface would rebound in such a way so as to strike the surface a second time, by considering the loss of energy and the rebounding angle of initially striking particles. These effects were included in the model by adding additional terms for the reduced rebound velocity and rebound angle in the equation of motion, which was solved numerically to obtain the resulting profiles. In addition, a spreading angle of the rebounding jet was incorporated into the model to smooth out the numerical effect of the second strike. The modified model showed an improvement over the initial one [22].

Both models (i.e., [21] and [22]) were developed for masked features only, using constant particle velocity and particle flux distributions. For unmasked features, the particle flux and spatial distributions play a fundamental role in feature prediction. Also, the models did not account for particles rebounding from the feature side walls and subsequently colliding with other particles, and interference effects between incident and rebounding particles. Moreover, the effects of 
particle size which affect the surface slope near the mask edge since not all particle sizes can locally contribute to the erosion process (Fig. 1.3), were ignored by the model of Ref. [22], whereas the model of Ref. [21] ignored the "second strike" effect. The above mentioned factors resulted in a disagreement between model and experiment. Both models ignored mask wear, which also affects the way the profiles evolve. Slikkerveer et al. [11] derived a simplistic semi-empirical model that explained elastomeric mask wear, but the effects of mask wear were not included in the surface evolution model and only qualitative conclusions were drawn (i.e., that erosion resistant masks should be used).

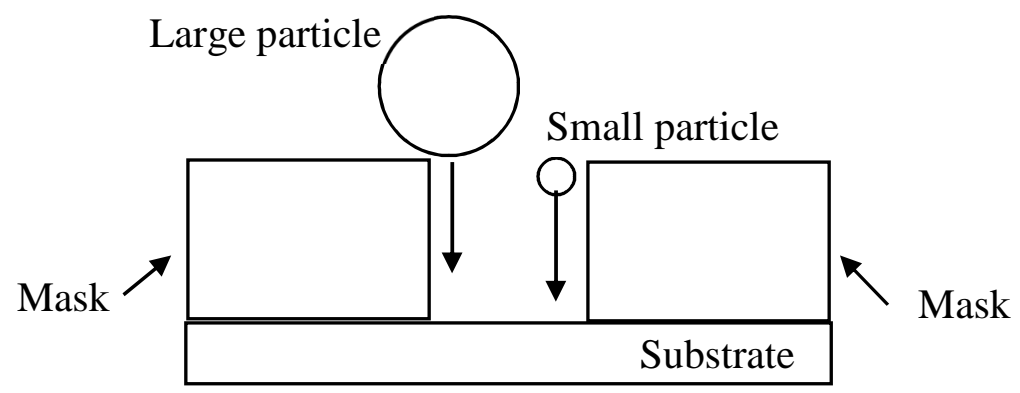

Fig. 1.3 A schematic demonstrating the effect of particle size on erosion of masked targets; large particle (relative to the mask opening) do not hit the target.

Moktadir et al. [24] extended the work of Slikkerveer et al. [22] by deriving a continuum equation based on the change of surface free energy due to particle impact that automatically included surface relaxation mechanisms (i.e., accounted for surface curvature effects) in glass targets. This work, along with Wensink et al. [25] and Wensink and Elwenspoek [13], showed that in the case of channel profiles machined in masked glass channels with aspect ratios up to 2.5, different shapes evolved. Firstly, a parabolic shape develops into a ' $V$ ' shape. Then, due to necking, a wider pocket near the bottom of the profile is developed for aspect ratios greater than 1. They stipulated that these shapes originated from a "blast lag" effect caused by interference between arriving particles and those rebounding from the mask edge, which resulted in side walls that are sloped as opposed to vertical. This could also be the result of the change of the shape of the channel itself. As the profile deepens, the "neck" prevents larger particles from reaching the bottom of the profile and collide with the side walls instead, [13], [22], [26]. The model was noteworthy, since the resulting profile shape matched experiments well due to the incorporated 
relaxation mechanism. Although their experiments explained the blast lag effect, their model could not account for particles rebounding from the feature side walls and particles colliding with other particles (i.e., interference effects between incident and rebounding particles)), effects of particle size, mask interference and mask wear, all of which can have a major influence on the way profiles develop.

In addition to the above work, other models for surface evolution in brittle substrates, such as glass, have been developed. Balasubramanian et al. [27] derived a simplistic analytical model for erosion rate as a function of profile radius which incorporated an exponential particle spatial distribution and a linear particle velocity distribution. Achtsnick et al. [28] and Karpuschewski et al. [17] developed a 1D isentropic flow model to measure the outlet velocity of single particles in the airflow for circular and square nozzles. Particle sizes and positions in the jet were based on normal probability distribution functions. Classical indentation fracture mechanics were used to model the erosion mechanism between the particles and the surface. These aspects were incorporated to obtain a particle jet characteristic energy intensity distribution. The above models are notable, but they did not consider the change in the normal component of the erosive energy as the slope of the surface develops, and can thus predict only low aspect ratio features (less than 0.5). In addition, these models have not been extensively verified against experimentally obtained masked profiles (which have high aspect ratios), and thus are questionable. Also, the models did not account for particles rebounding from the feature side walls and particles colliding with other particles, effects of particle size, mask interference and mask wear, all of which can have a major influence on the way profiles develop.

Ghobeity et al. [9]. adopted the semi-empirical surface evolution model of BoonkkampJansen [21] which was proposed for brittle materials. Many of the limitations were addressed by introducing considerable modifications. The governing equation describing the surface evolution for a feature-cross section of [21] was:

$$
z_{, t}-\frac{C}{\rho_{s}} V(x)^{k} \varphi(x)\left(1+z_{, x}{ }^{2}\right)^{-k / 2}=0
$$

where $z_{, t}$ and $z_{x}$ are the partial derivatives of the profile depth $z$ as a function of exposure time and the coordinate $x$, defining the width dimension. $\phi(x)$ and $V(x)$ are the particle mass flux and velocity 
distributions, respectively, $C$ is an erosion constant, $k$ is the velocity exponent and $\rho_{s}$ is the density of the substrate. Furthermore, for a scanning nozzle, the particle mass flux and velocity distributions would also be a function of scanning time and scanning velocity. It was shown that the eroded profile generated across the diameter of a stationary nozzle was equivalent to the one which was generated by a scanning nozzle, provided that the erosion rate was not significantly high and the scanning speed was not significantly slow. Unlike [20] and [21] which determined the dependence of erosion rate on particle velocity, the model of [9] proposed considering the dependence of erosion rate on only the normal component of the velocity and thus the erosion ratevelocity dependence was determined by varying the angle of attack. This eliminated the necessity of measuring the erosion rate on particle velocity experimentally which was a complicated and time consuming procedure.

However, Ref. [20] still over-predicted the centerline depths of the channels for both masked and unmasked machining. This failure was likely due to a number of reasons: 1) the model assumed that the scanning nozzle was equivalent to a stationary nozzle; 2) it neglected particle interference effects; 3 ) it neglected the "second strike" effect. To improve upon this, the mask edge effect was modeled by fitting an exponential function for the net erosive power or 'erosive efficacy' (product of velocity raised to the power $k$ and the particle mass flux) in Eq. (1.6) from the experimentally obtained first pass profile for both masked and unmasked channels. These results were confirmed using a particle trajectory computer model that takes into account particle "second strike effect" due to the edge of mask, mask thickness and particle size [26]. The results showed significant improvements for aspect ratios up to 0.5 over previous models of Refs. [21] and [22]. This approach made the analysis less complicated. However, a true physical model would have to be a function of the particle size and shape, velocity as a function of time, mask height, as well as particle rebound and interference characteristics. In addition, the model could not be extended to work for oblique incidence, where the resulting profiles are asymmetrical, and hence 3D cases, and could not account for mask wear.

The model of Ref. [9] was extended to work for ductile materials such as PMMA [6]. the resulting surface evolution model for ductile martials was given as:

$$
z,-\frac{C}{\rho_{s}} V(x)^{k} \varphi(x) \sqrt{1+z,{ }_{x}{ }^{2}} g(\alpha)=0
$$


where $\mathrm{g}(\alpha)$ is the semi-empirical function given in Eq. (1.4). The model was now a more complex function of the impact angle and substrate hardness, determined experimentally, which could model the ductile erosion process. The model predicted the centerline depth up to an aspect ratio 0.25 for unmasked channels and aspect ratio of 0.6 for masked channels, but it successively overpredicted the channel width with each pass [6]. Getu et al. [6] used a thermocouple to measure the temperature change as AJM was performed on a PMMA target under typical conditions. It was observed that the temperature increase was less than $8{ }^{\circ} \mathrm{C}$ which therefore did not affect the erosion behavior of the PMMA.

Ghobeity et al. [29] developed a relatively simple model to estimate the erosive efficacy across the mask opening in the machining of micro-holes and micro-channels in glass and PMMA. An analytical model was derived for machining at normal incidence that could be implemented to evaluate the erosive efficacy close to the mask edge as a function of the size of the mask opening and the measured normal and log-normal particle size distribution. The model was based on an expression of the proportion of the total particle mass as a function of the particle radii, which accurately described the effect of mask edge on the particle mass flux distribution of the particles passing through the mask opening.

The predictions matched the experimental channels very well the aspect ratios up to 2 in glass and up to 0.2 in PMMA. For higher aspect ratio channels machined in glass the fits were poor due to the inability of the model to take into account the second strike effect; and for the case of machining PMMA channels, neglect of particle embedding in the model resulted in poor results. The most practical aspect of the model was prediction of evolution of micro features profiles without using semi-empirical or computer particle tracking techniques. However, it was only derived for normal incidence cases and thus could not be used to study oblique incidence and hence three-dimensional erosion in AJM. In addition, the model did not account for the second strike effect and mask wear, which can further affect the resulting profile shape near the mask edges.

Ally et al. [30] subsequently implemented the surface evolution equation of [6] to predict the shapes of low aspect ratio unmasked channel profiles machined in flat metal targets at oblique incidence. First the erosive efficacy (the velocity and mass flux distributions) was obtained across the jet by curve fitting to a shallow channel machined at normal incidence; subsequently, a coordinate transformation was adopted that mapped the erosive efficacy at a coordinate normal to the jet axis to the tilted surface coordinate. It was observed that the model resulted in an 
overestimation of the channel width, which, as will be shown in Chapter 3, could be attributed to the neglect of the greater effect of jet divergence on the local impact angles and standoff.

Burzynski and Papini [31] developed a level-set model (LSM) to predict the surface evolution of high aspect-ratio masked and unmasked channels and holes machined into glass and PMMA flat targets at normal and oblique incidence, and considering particle second strikes. This model accounted for the change in the standoff distance and spread in particle trajectories which made it capable for predicting very deep channel profiles. In the case of tilted channels, the origin of the coordinate system was moved from the target surface to the nozzle exit. This simplified the formulation since the complex transformations of particle trajectories was made unnecessary. The predictions of the model were generally in good agreement with experiments, and the model was able to shed light on the role of second strike and other complex phenomena when eroding high aspect ratio features onto flat surfaces. However, such LSM formulations are quite complex, and difficult to routinely implement.

\subsubsection{Micro-machining of highly curved targets}

To the knowledge of the author, the AJM of stationary and rotating highly curved glass (brittle) and PMMA (ductile) materials has not received attention, neither in fabrication nor in modelling. However, other machining methods have been utilized for machining features on curved targets, i.e. rods. For instance, Matsumura and Ueki [32] used a ball-end mill to make 20 $\mu \mathrm{m}$ deep grooves on $6 \mathrm{~mm}$ diameter cylindrical glass rods. Furutani et al. [1] used electro-chemical discharge machining as a lathe for machining on $5 \mathrm{~mm}$ glass rods. Abrasive water jets (AWJ) have been also studied widely as lathes. For example, Li et al. [33] studied the AWJ turning process on $59 \mathrm{~mm}$ diameter steel rods, and investigated the effects of different process parameters on the depth of cut. Similarly, Zhong and Han [34] investigated the effect of AWJ process parameters in the turning of glass but on the surface finish. Manu and Babu [35] implemented Finnie's theory of erosion to model the material removal from a $25.4 \mathrm{~mm}$, rotating 6063-T6 aluminum alloy rod using abrasive waterjet turning with the nozzle displaced radially from the rod axis so that the erosion was tangential, focusing at the edge of the rod. The presented model neglected the particle spread in the jet, resulting in an error of about 10\%. Carach et al. [36] used a tangential abrasive waterjet $(\mathrm{AWJ})$ as a lathe to machine Incoloy alloy 925, an alloy that is difficult to machine 
conventionally because of rapid tool wear. They investigated the effect of traverse speeds on the surface roughness and realized that higher speed resulted in higher roughness. In summary, AJM is an effective technique for machining micro-features in both brittle and ductile materials. AJM models and machining procedures presented in the literature are limited to flat targets and mostly at normal incidence. However, there is a demand for micro features to be machined on curved surfaces, especially for biomedical and microfluidic applications. This shortcoming forms the primary motivation for this dissertation.

\subsection{Objectives}

The main objective of this dissertation is to investigate the abrasive air jet micro-machining of stationary and rotating highly curved targets. The following secondary objectives will serve as milestones to achieve this main objective:

$>$ Design, development and testing of a new AJM direct-write masking technique (Chapter 2).

$>$ Development of an analytical model to predict the shape of micro-channels machined on highly curved (i.e., a surface in which the difference between the local standoff at the edge of the jet footprint and at the centerline is at least $5 \%$ of the standoff at the jet axis) glass and PMMA surfaces (rods), (Chapter 3).

$>$ Prediction of erosive footprint in AJM of flat and curved glass targets by studying the effects of initial impacts and second strikes of rebounding particles on the size of footprint (Chapter 4).

$>$ Development of a model for the prediction of material removal during the machining of rotating and translating PMMA and glass rods (Chapter 5).

$>$ Development of a novel experimental procedure for micro-machining helical channels in glass and PMMA rods. 


\section{Chapter 2 Implementation of a shadow mask for direct writing in abrasive jet micro-machining}

This chapter is based on the following published paper:

A. Nouhi, M.R. Sookhak Lari, J.K. Spelt, M. Papini, "Implementation of a shadow mask for direct writing in abrasive jet micro-machining", Journal of materials processing technology, vol. 223, pp. 232-239, 2015.

* The first two authors contributed equally to this paper.

\subsection{Introduction}

Abrasive jet micro-machining (AJM) uses compressed air to create a jet of small particles to machine small-scale features in, for example, micro-fluidic, micro-electromechanical systems (MEMS) and opto-electronic components. AJM is attractive because of its low capital and operating cost, its very high etch rate on brittle materials such as glass, its ability to machine a wide range of materials, and its directional etch capability. Because the size of the jet footprint is typically on the order of several millimeters, a potential drawback of AJM is the need to apply erosion resistant masks in order to etch micro-scale features. Conventionally, the jet of particles is directed through an erosion resistant mask opening that is patterned to define the required features.

A number of devices have been recently fabricated using AJM in conjunction with patterned masks attached to the surface. For example, Belloy et al. [37] fabricated glass inertial sensors, Schlautmann et al. [38] electrophoresis capillaries, Park et al. [39] micro-molds in steel, and Yamahata et al. [40] ball valve micro pumps in glass. Masaki et al. [41] added a circular vane to slot nozzles in order to achieve a more uniform particle velocity distribution in order to machine large areas.

A number of different materials have been used to fabricate patterned masks. For example, Lomas et al. [42] developed a precision hot embossing metal mold in stainless steel by highresolution powder blasting in which PDMS and SU-8 were used as masks. Pawlowski et al. [14] used photolithographic methods to apply either epoxy or polydimethylsiloxane masks to glass substrates. This allowed feature sizes of $20-30 \mu \mathrm{m}$ to be achieved when blasting with $10 \mu \mathrm{m}$ diameter aluminum oxide particles. They also observed a reduction in the etching rate as the mask opening become smaller than ten times the particle size. Wensink et al. [20] also tested two 
polymers which could be lithographically structured on glass, and investigated the use of electroplated copper applied to glass over a titanium binding film. The resulting copper mask was highly erosion resistant, and channels of less than $50 \mu \mathrm{m}$ width were achievable. Liao and Chen [43] used various lithographic approaches to manufacture erosion resistant masks on glass and Ghobeity et al. [44] achieved feature sizes as small as 20-50 $\mu \mathrm{m}$, and channel aspect ratios of approximately 1 by using this method. Ghobeity et al. [9] also utilized a clamped mask consisting of two parallel $3 \mathrm{~mm}$ thick tempered steel strips in order to make straight channels in glass plates. The steel strips were milled to give sharp $90^{\circ}$ corners and smooth edges bounding the masked region. The use of these relatively thick steel strips improved channel edge definition by minimizing mask undercutting, providing greater resistance to lifting by the air stream and decreasing the number of particles striking the target surface at oblique angles after scattering from the corner edges of the mask.

As described above, conventional masks are either bonded or clamped to the target substrate. This limits flexibility and increases costs, since each device design requires a unique mask. This paper introduces a new adjustable shadow mask system which is attached to a stationary nozzle, producing a narrow stream of abrasive particles to machine a target which is scanned in front of it, in order to produce the desired pattern. This avoids the cost and inconvenience of manufacturing, applying and removing fixed masks, as is the need for chemical solvents. The present work investigated the effects of shadow mask and nozzle stand-off, particle mass flux, and particle size on the resolution of the resulting features.

\subsection{Experiments}

\subsubsection{Shadow mask apparatus}

All experiments were conducted using an AccuFlo AF10 Micro-Abrasive Blaster, (Comco, Inc. Burbank, CA, USA) operating at $200 \mathrm{kPa}$ air pressure. A dehumidifier, a refrigeration air dryer, and a desiccant filter on the inlet air were used to minimize the moisture in the powder reservoir. A variable speed rotary electric mixer (Arrow Model 850, Arrow Engineering Co., Inc., Hillside, NJ, USA) was used in the reservoir to avoid powder clogging and particle agglomeration, and the powder level inside the reservoir was kept constant in order to reduce fluctuations in particle mass flux based on Ghobeity et al. [45]. 
Aluminum oxide $\left(\mathrm{Al}_{2} \mathrm{O}_{3}\right)$ powders with nominal diameters of $10 \mu \mathrm{m}$ (mean: $11.6 \mu \mathrm{m}$, standard deviation: $3.31 \mu \mathrm{m}$ ) and $25 \mu \mathrm{m}$ (mean: $24.1 \mu \mathrm{m}$, standard deviation: $16.2 \mu \mathrm{m}$; Comco, Inc. Burbank, CA, USA) were blasted on $3.3 \mathrm{~mm}$ thick borosilicate glass targets (Borofloat, Swift Glass Co. Inc., Elmira, NY, USA). The glass samples were clamped to a programmable computercontrolled linear stage (Aerotech Inc., Pittsburgh, PA, USA) with a positioning resolution of 0.5 $\mu \mathrm{m}$. The stage permitted independent motion in two directions and could be used to provide curvilinear motion of the target under the fixed jet.

For the machining of straight channels, the clamp shown in Fig. 2.1 was designed to hold the shadow mask and nozzle as the target was moved past them. A set of 3 equally-spaced springs between the nozzle and mask holder maintained a uniform contact pressure between the mask and the target surface. The nozzle was attached to the apparatus using a set screw, and the mask was clamped to the apparatus, in order to keep the jet centerline normal to the shadow mask and target. The nozzle-to-surface standoff was maintained at $20 \mathrm{~mm}$ unless otherwise mentioned (Fig. 2.1). A relatively large $0.3 \times 3.8 \mathrm{~mm}$ rectangular nozzle (Comco, Inc. Burbank, CA, USA) was used with its long axis perpendicular to the slot of the mask opening (Fig. 2.2) in order to minimize the effect of small misalignments of the jet center.

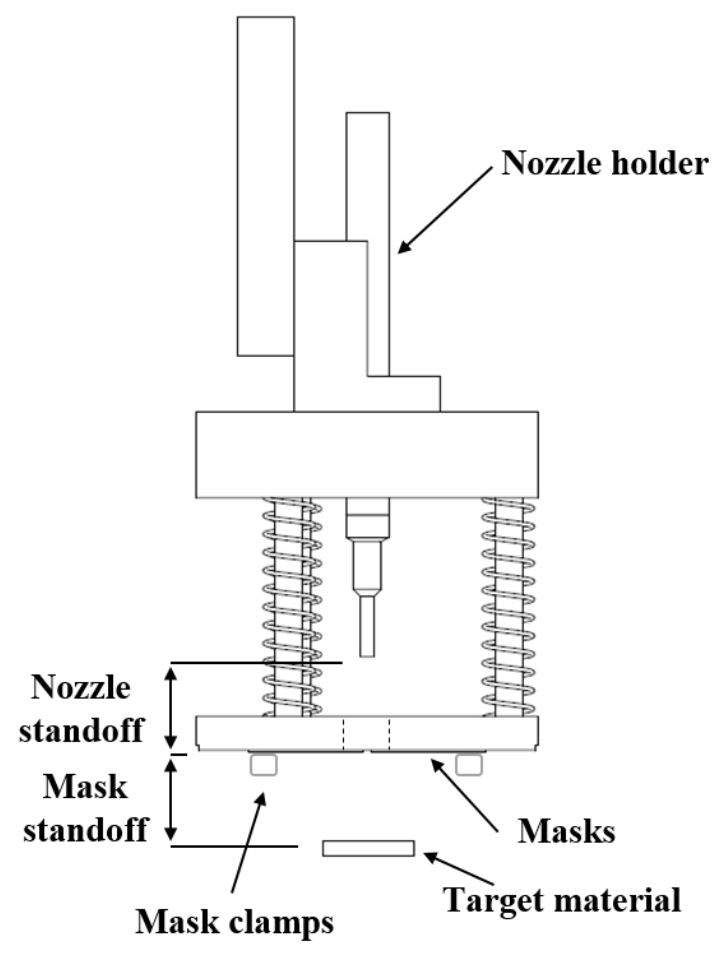


Fig. 2.1 Shadow mask apparatus. Nozzle standoff $(N S)$ and mask standoff $(M S)$ were defined relative to the top of the mask. Only two of three springs shown.

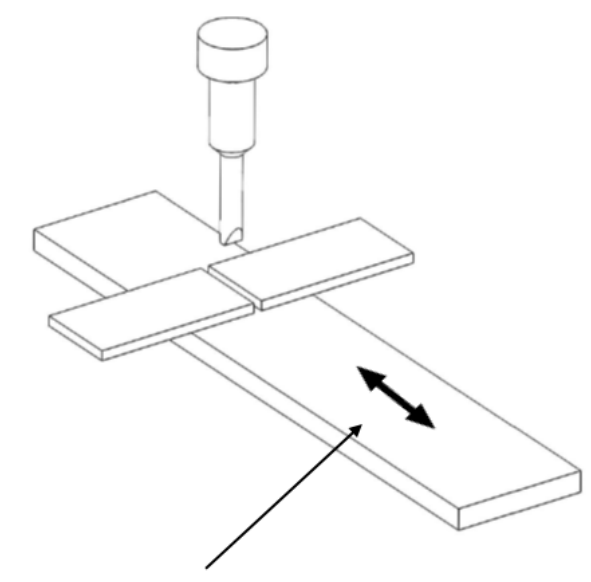

Direction of movement of target

Fig. 2.2 Relative orientation of nozzle and shadow mask.

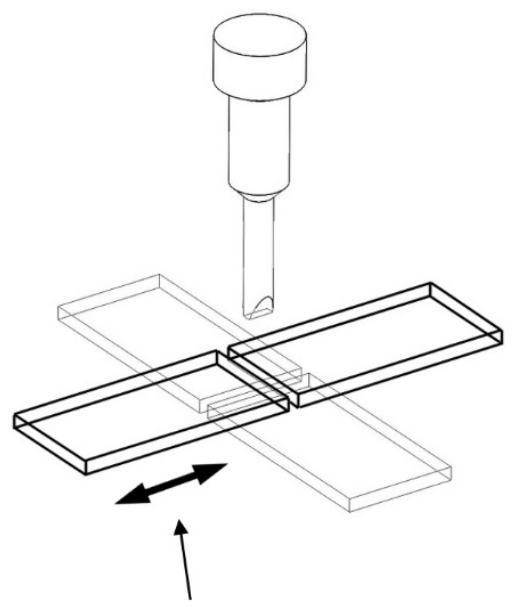

Direction of movement of target

Fig. 2.3 Jet aperture for direct writing of curved paths made using two sets of crossed shadow masks. 
The desired mask opening was obtained by placing a steel sheet of known thickness between the movable masks, and the clamping them in place. The mask opening was then photographed to determine its width with an accuracy of $\pm 6 \mu \mathrm{m}$ using digital image analysis. The mask openings for all experiments were in the range of 250 to $300 \mu \mathrm{m}$. Linear channels of $30 \mathrm{~mm}$ length were machined by moving the glass target under the mask at various mask standoff $(M S)$ and nozzle standoff (NS) distances (Fig. 2.1) in a straight line at a traverse velocity of $1 \mathrm{~mm} / \mathrm{s}$. All experiments under a given set of conditions were repeated on three specimens.

The mass flow rate before and after each experiment was measured by weighing the abrasive collected after 2 min of blasting into a cylinder covered with an air filter. The change in the mass flow rate during the course of a 2.5 min experiment was less than $10 \%$.

A final set of experiments was conducted using two sets of crossed masks to provide a square-shaped mask opening (Fig. 2.3). This was used to machine linear and curvilinear channels. Table 1 summarizes the conditions used in the various experiments.

\subsubsection{Measurement of channel cross-section and frosted region}

The channels were scanned using an optical profilometer (NANOVEA ST400 Micro Photonics Inc., Irvine, CA, USA) having a depth resolution of $25 \mathrm{~nm}$ and a lateral resolution of $0.1 \mu \mathrm{m}$, in order to determine the effect of various process parameters on the resulting channel dimensions and shape. It was found that the glass adjacent to the edges of the channels was slightly eroded ("frosted") as illustrated in Fig. 2.4, likely due to particles penetrating under the masks. The width of this frosted region was measured using image analysis of photomicrographs as explained below. The erosion in the frosted regions was too small to produce a significant change in the average elevation of the glass target.

The frosted region complicated the definition of channel width. In the present work, the channel edge was defined as the point at which the average eroded depth of 10 consecutive profilometer measurement points, each $0.5 \mu \mathrm{m}$ apart, was equal to the depth of a crater created by a single particle of average size. According to Slikkerveer et al. [46] and by referring to Marshall et al. [47], the depth $a$ of a hemispherical crater is given by:

$$
a=\left(\frac{3 U}{2 \pi H}\right)^{\frac{1}{3}}
$$


where $H$ is the Vickers hardness of the target, and, $U$, the kinetic energy, is

$$
U=\frac{1}{2} \rho\left(\frac{4}{3} \pi R^{3}\right) v^{2}
$$

and $\rho, R$ and $v$ are the density, radius and velocity of an average erodent particle, respectively. Substituting Eq. (2.2) into Eq. (2.1), the depth of the indented zone becomes

$$
a=R\left(\frac{\rho v^{2}}{H}\right)^{\frac{1}{3}}
$$

For borosilicate glass, the Vickers hardness is $5.68 \mathrm{GPa}$ and the density of aluminum oxide is $3,950 \mathrm{~kg} / \mathrm{m}^{3}$. Assuming a particle diameter $d=25 \mu \mathrm{m}$, the particle velocity model of Li et al. [48] was used to estimate an average particle velocity of $150 \mathrm{~m} / \mathrm{s}$ at a nozzle to surface standoff distance of $20 \mathrm{~mm}$. These values result in predicted crater depth $a=3.15 \mu \mathrm{m}$, which is approximately equal to $1 / 8^{\text {th }}$ of the particle diameter. Thus, the edge of the channel was considered as the point where the eroded depth was equal to $d / 8$, and the channel width was the average of three cross-sectional profiles, each on three separate specimens (9 measurements).

As shown in Fig. 2.4, this definition of channel width typically agreed to within $10 \%$ with that obtained using a second method (slope method) whereby the edge of a channel was defined as the point, beginning from the sidewall of a channel and moving outward toward the periphery, where the absolute slope of a straight line fitted to 5 consecutive points spaced $5 \mu \mathrm{m}$ apart along a given channel cross-section was less than $10 \%$. Since the crater size method was easier to implement and was less sensitive to the resolution of the scanned profiles, it was used throughout the paper. 


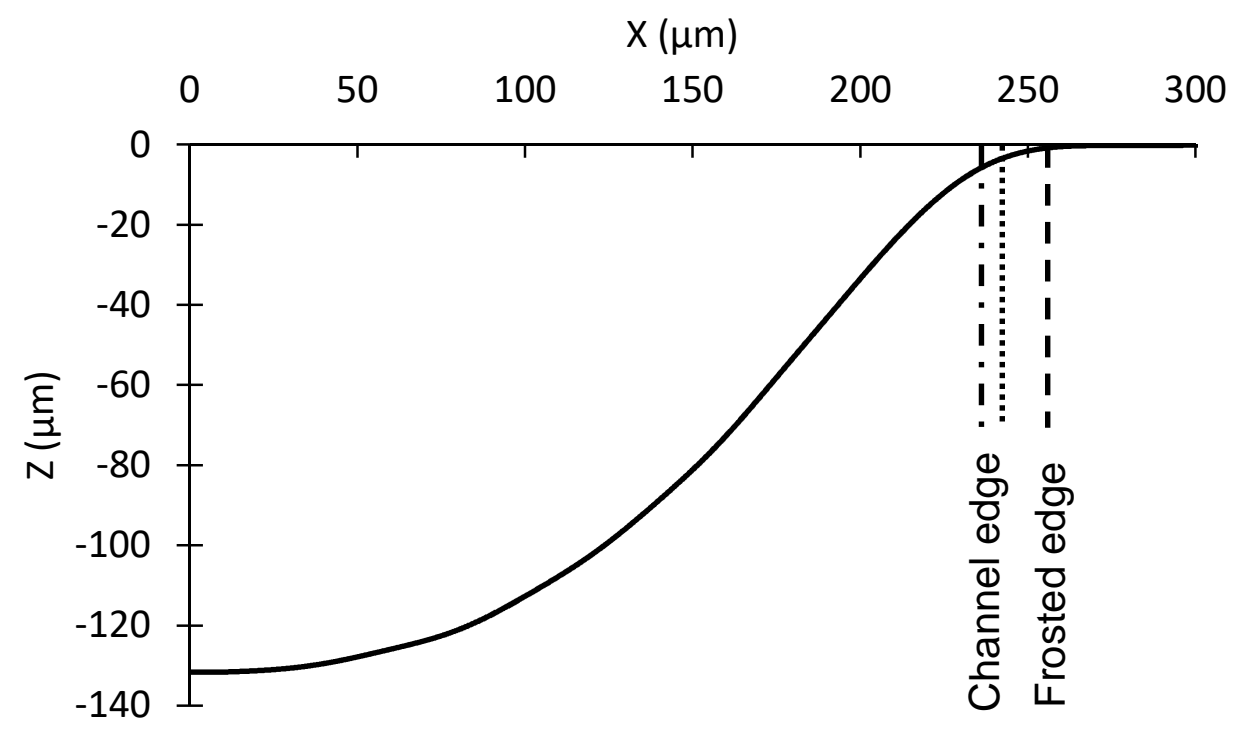

Fig. 2.4 Location of edge of frosted area (dashed line) and a comparison of channel width based on (i) the slope method (dashed dotted line) and (ii) the channel crater size method (dotted line).

In order to obtain a measure of the width of the frosted region, three distinct subregions in a complete image of a channel (Fig. 2.5) could be roughly recognized by differences in their color; i.e. an uneroded region relatively far from the channel (lightest), a frosted zone (grey), and finally the channel itself (dark grey). Once the channel width for a given channel was determined as described above, it was centered within the dark area, as shown in Fig. 2.5 (the innermost two lines representing the channel width), and all pixels within the channel width were assigned totally black greyscale values. The outer edges of the frosted region were determined by performing image segmentation using Matlab (The Mathworks, Inc.), adjusting the threshold greyscale value until the greyscale of all pixels in the uneroded region were visually judged to be included. The pixels in this segmented area, which represented the uneroded area, were then also set to black. This resulted in the frosted regions being white, while the channel and surrounding uneroded glass were black, as shown in Fig. 2.6. The average width of the white areas was then determined by multiplying the image width (vertical dimension of Fig. 2.6) by the ratio of the white to black areas. Finally, the width of the frosted zone on each side of a channel was considered as half of the measured total width. The reported frosted widths were the average of the values on 3 separate specimens. Fig. 2.4 shows the typical location of the frosted edge on a cross-sectional profile. 
While these definitions were somewhat subjective, they provided a consistent means to assess the dependence of the channel and frosted area widths on the process parameters.

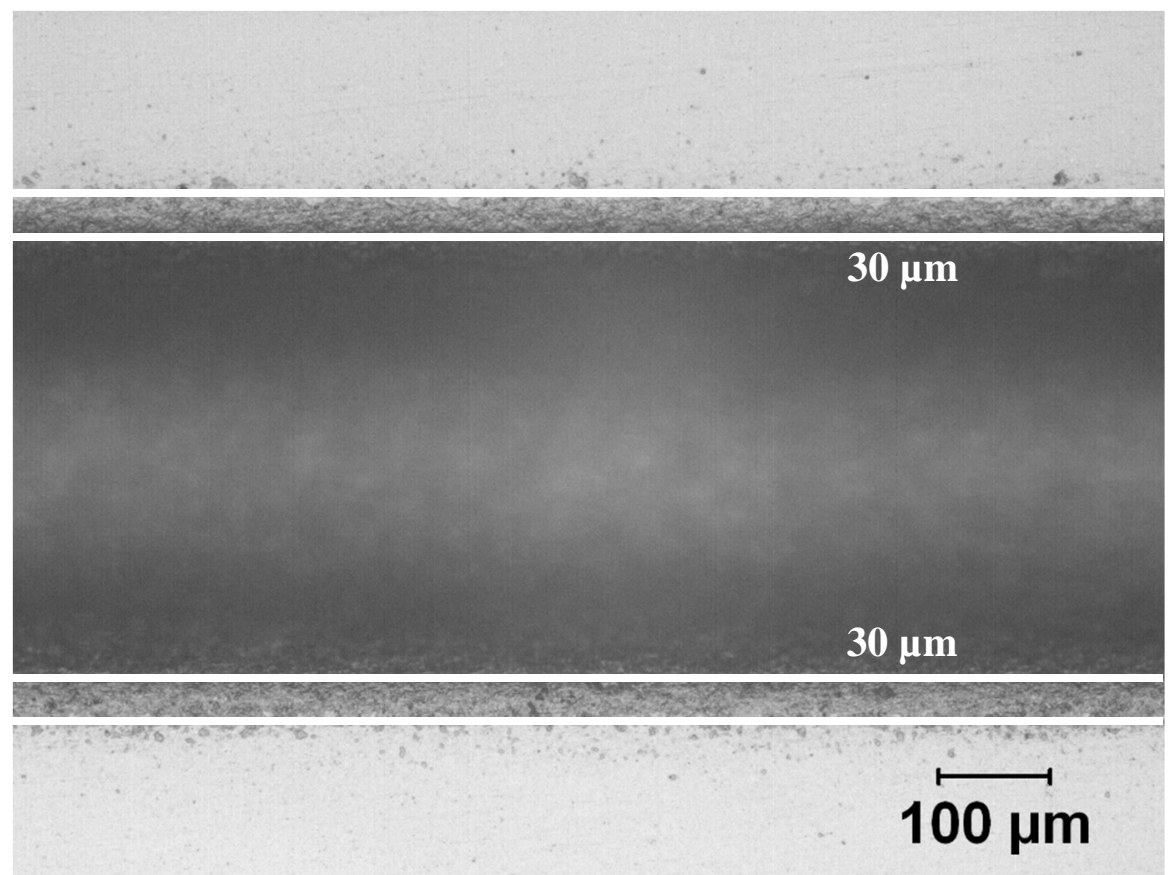

Fig. 2.5 Top view of glass channel blasted at $200 \mathrm{kPa}, 20 \mathrm{~mm}$ nozzle standoff, with a contacting crossed shadow mask having a square opening $260 \mu \mathrm{m}$ on a side. The $30 \mu \mathrm{m}$ wide frosted regions are indicated. 


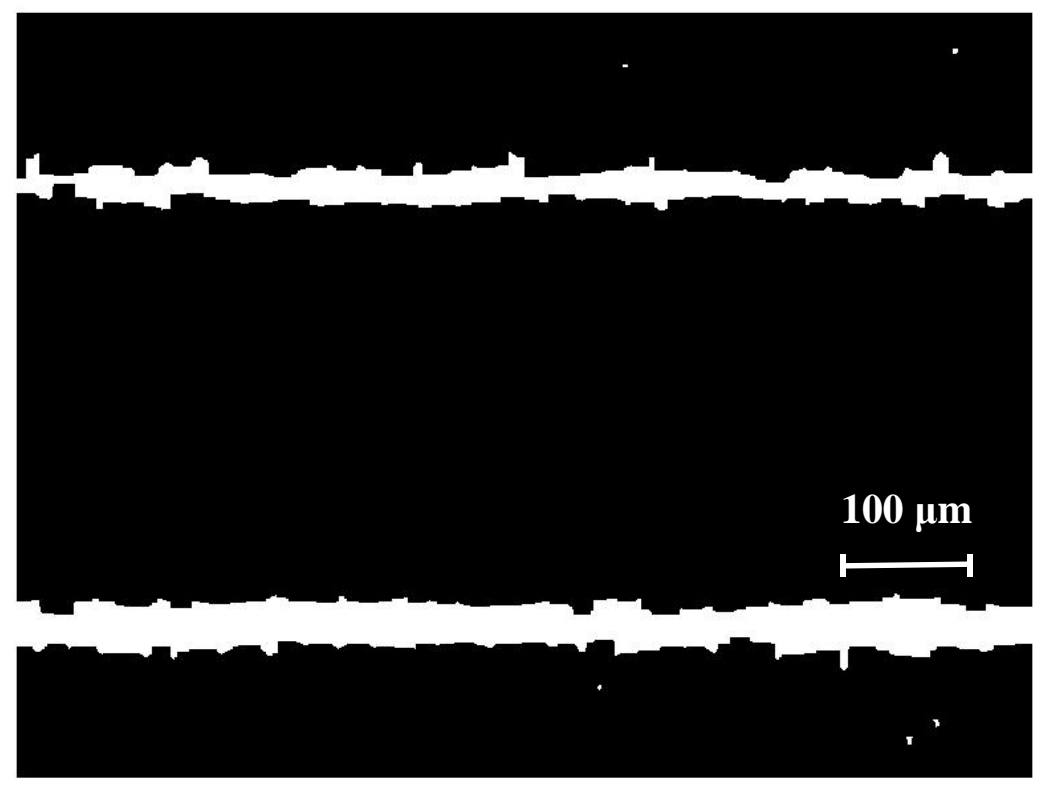

Fig. 2.6 Segmented image of Fig. 2.5 showing the frosted regions in white. 
Table 2. 1 Summary of conditions used in each experiment.

\begin{tabular}{|c|c|c|c|c|c|c|c|}
\hline Experiment & $\begin{array}{c}\text { Particle size } \\
(\mu \mathrm{m})\end{array}$ & $\begin{array}{c}\text { Pressure } \\
(\mathrm{kPa})\end{array}$ & $\begin{array}{c}\text { Mask opening } \\
(\mu \mathrm{m})\end{array}$ & $\begin{array}{l}\text { Powder } \\
\text { mass flow } \\
\text { rate }(\mathrm{g} / \mathrm{min})\end{array}$ & $\begin{array}{c}\text { Nozzle } \\
\text { standoff }(\mathrm{cm})\end{array}$ & $\begin{array}{c}\text { Mask } \\
\text { thickness } \\
(\mathrm{mm})\end{array}$ & $\begin{array}{l}\text { Mask } \\
\text { standoff } \\
(\mathrm{mm})\end{array}$ \\
\hline Clamped vs. shadow mask & 25 & 200 & 293 & 6.1 & 2 & 1.6 & 0 \\
\hline Effect of nozzle stand-off distance $(N S)$ & 25 & 200 & 270 & 5.9 & $1-2-3$ & 1.6 & 0 \\
\hline Effect of mask thickness & 25 & 200 & 270 & 6.2 & 2 & $0.8-1.6$ & 0 \\
\hline Effect of mask standoff $(M S)$ & 25 & 200 & 270 & 4.6 & 2 & 1.6 & $0-2$ \\
\hline Effect of particle size & $10-25$ & 200 & 290 & 4.7 & 2 & 1.6 & 0 \\
\hline Crossed-mask linear channels & 25 & 200 & $290 \times 290$ & 5.6 & 2 & 1.6 & 0 \\
\hline Crossed-mask curvilinear channels & 25 & 200 & $575 \times 575$ & 5.4 & 2 & 1.6 & 0 \\
\hline
\end{tabular}




\subsection{Results and discussion}

\subsubsection{Clamped vs. shadow mask}

Fig. 2.7 compares the measured cross-sections of typical channels made using either the shadow mask of Fig. 2.2 sliding on the glass surface (i.e. mask standoff=0 in Fig. 2.1) or a traditional steel mask that was clamped to the glass. In both cases the mask opening was $293 \mu \mathrm{m}$, the mask thickness was $800 \mu \mathrm{m}$, and the nozzle standoff was NS=20 mm. It is evident that the shadow mask produced slightly wider and shallower channels than did the clamped mask. Depending on the number of passes, the channels machined using the shadow mask were approximately $3-15 \%$ wider and 2-24\% shallower than those using made with the clamped masks. This widening occurred because particles could penetrate under the shadow mask and strike the surface between the mask and the target, something that is less likely to happen with clamped masks. Even though the shadow mask was pressed against the target, it had a standoff equal to approximately the diameter of the largest particles in the abrasive mixture, since these particles became trapped under the contacting shadow mask as the target surface moves. The smaller particles in the powder were then able to strike the surface under the shadow mask creating the frosted zone.

It is hypothesized that the channels were shallower when using the shadow mask for similar reasons; i.e. the leakage of particles and air under the masks resulted in fewer particles striking the surface within the mask opening. While there was no noticeable frosting of the glass at the periphery of the channels when the mask was clamped, the shadow mask produced frosted bands on either side of the channel that were approximately $60 \mu \mathrm{m}$ wide, because particles could penetrate under the mask. 


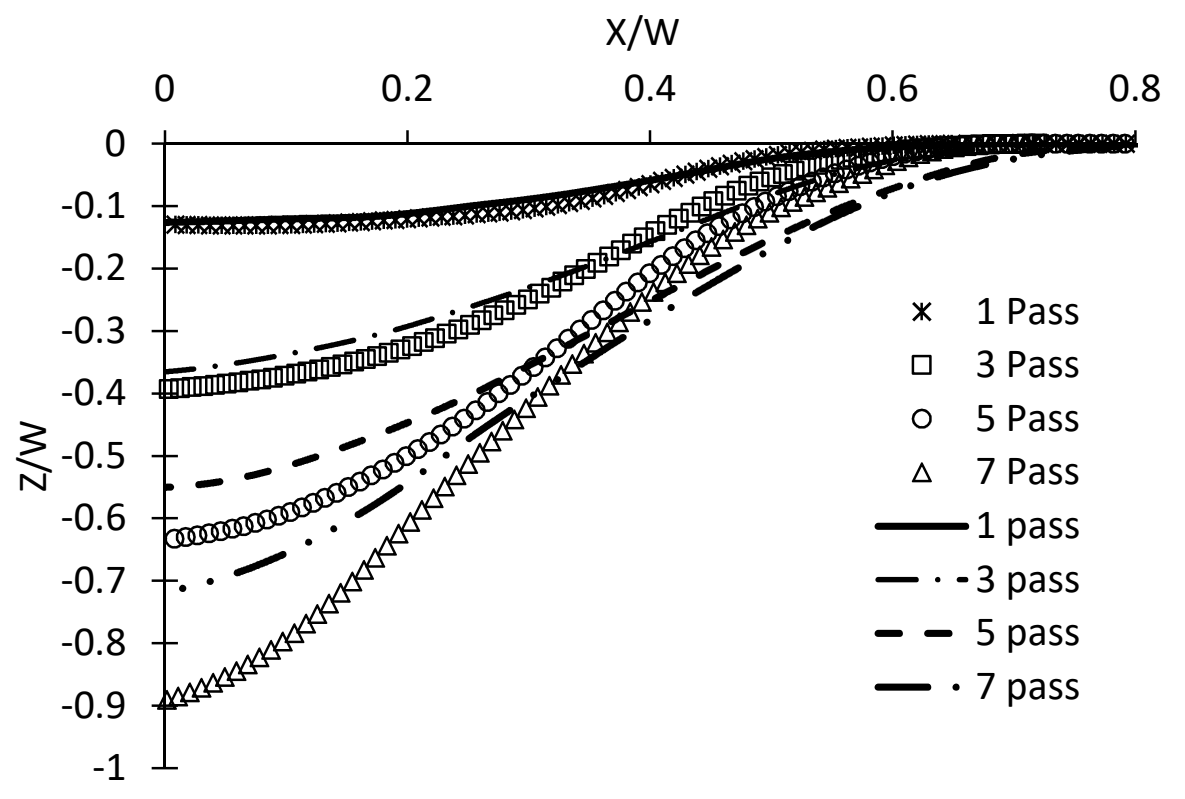

Fig. 2.7 Cross-sectional profiles of channels machined using various number of passes and the shadow mask of Fig. 2.2 in contact with the surface (solid and dashed lines) or a clamped mask

(symbols). Dimensions normalized using the mask opening, $W=293 \mu \mathrm{m}$. Only half of each symmetric profile is shown. Mask thickness of $800 \mu \mathrm{m}, N S=20 \mathrm{~mm}$.

\subsubsection{Effect of nozzle standoff distance}

The effect of the nozzle standoff distance was investigated for the shadow mask of Fig. 2.2 having an opening of $270 \mu \mathrm{m}$ with the mask and surface in contact (i.e. $M S=0$ ), and $N S=10,20$ and $30 \mathrm{~mm}$ (Fig. 2.1; conditions of Table 1). Fig. 2.8 and Fig. 2.9 show that increasing NS from $10 \mathrm{~mm}$ to $30 \mathrm{~mm}$ resulted in approximately $8 \%$ shallower and $7 \%$ narrower channels, and a $20 \%$ decrease in the width of the frosted region, Fig. 2.10. The changes in channel depth, width and frosting were relatively small, but statistically significant ( $t$-test, $\mathrm{p}<0.05$ ). These effects were attributable to the reduction in the particle flux through the mask opening that occurred with increasing nozzle standoff distance due to the divergence of the jet plume which can be found in work presented by Papini et al. [49]. This resulted in less material removal, both in the center and at the periphery of the channel, and produced fewer particles undercutting the mask edge to form the frosted regions. 


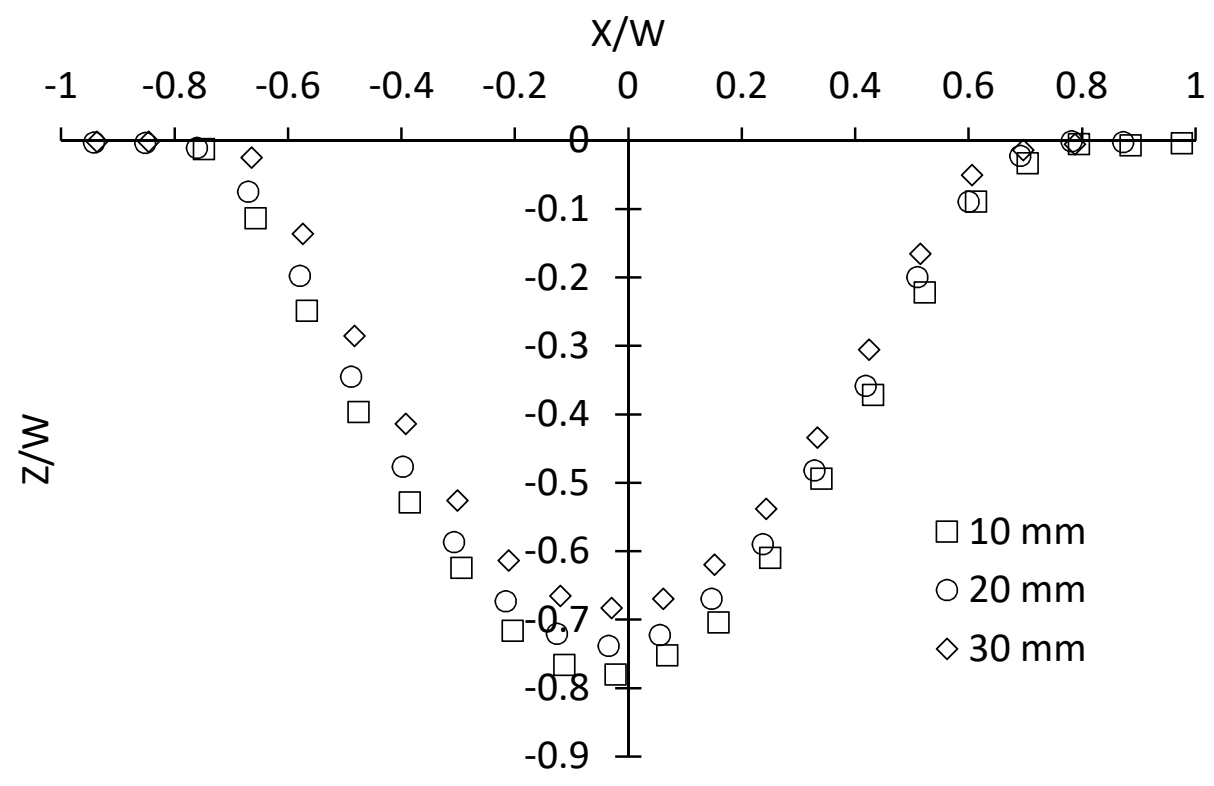

Fig. 2.8 Comparison of typical channel profiles obtained after 4 passes of the shadow mask of Fig. 2.2 at three nozzle standoff distances (Fig. 2.1). Depth and width normalized by mask opening $W=270 \mu \mathrm{m}$.

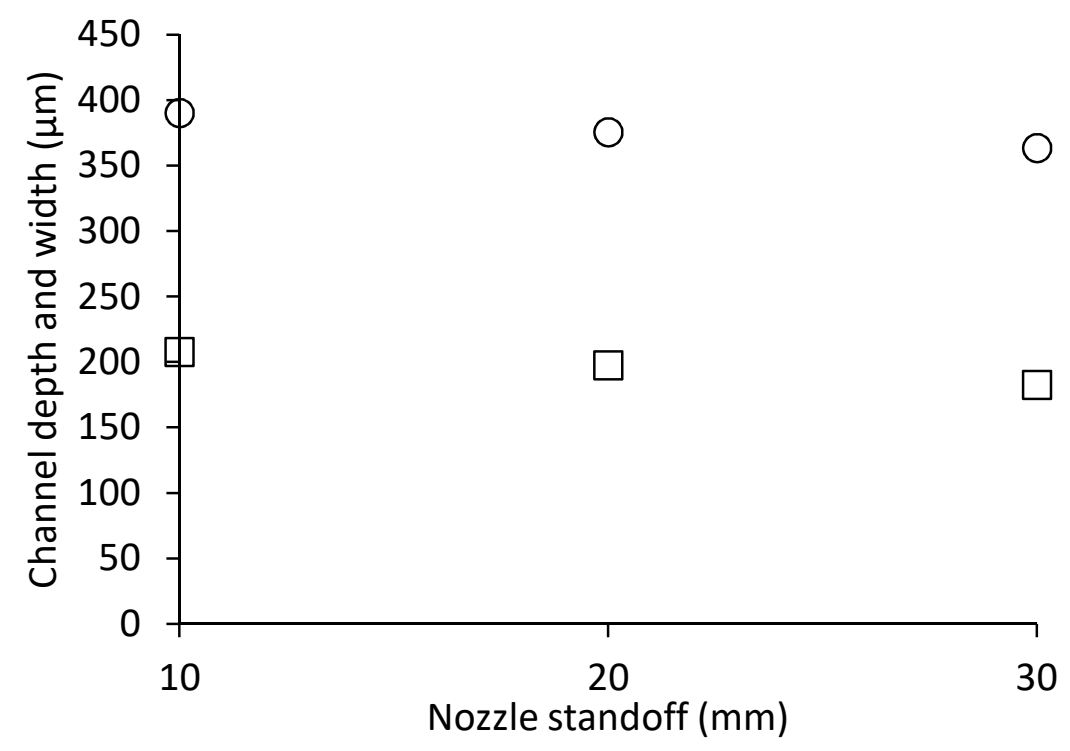

Fig. 2.9 Average channel depth (squares) and width (circles) at three nozzle standoffs. Scatter bars representing \pm 1 standard deviation for 9 measurements ( 3 measurements on each of 3 specimens) were small enough to fit within the symbols. 


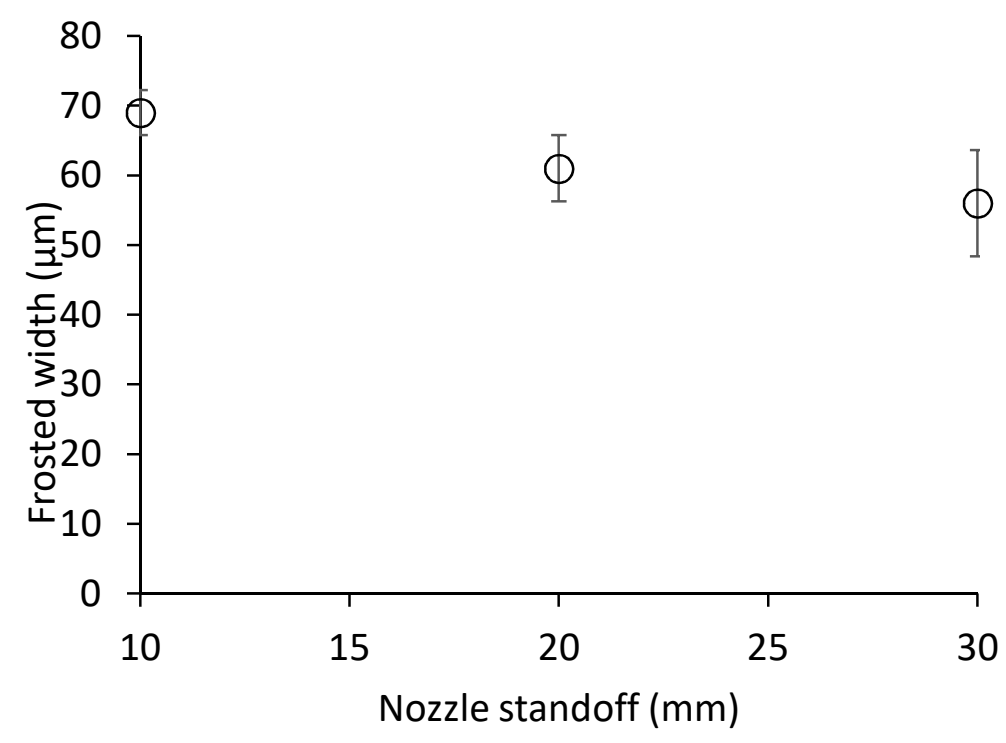

Fig. 2.10 Average frosted width at three nozzle standoffs (Fig. 2.1). The scatter bars represent \pm 1 standard deviation for 9 measurements ( 3 measurements on each of 3 specimens).

\subsubsection{Effect of shadow mask thickness}

The effect of shadow mask thickness on the channel profiles was investigated using 0.8 and $1.6 \mathrm{~mm}$ thick contacting shadow masks of the type shown in Fig. 2.2, with an opening of 270 $\mu \mathrm{m}$. Fig. 2.11 and Fig. 2.12 show that the thicker mask produced statistically significant decreases (t-test, $\mathrm{p}<0.05)$ in both the channel depth $(17 \%$ decrease) and width ( $4 \%$ decrease). Figure 2.12 shows that the frosting width also decreased, by more than two fold when the mask thickness increased. These trends can be attributed to a reduction in the particle flux capable of erosion due to differences in particle ricochet from the mask edges, as shown in Fig. 2.13. In the case of the thicker mask, it is more likely that particles which strike the edge of the mask will ricochet onto the opposite mask and lose most of their kinetic energy. Thinner masks permit more of the incident particles to reach the target surface with sufficient kinetic energy to produce erosion. Therefore, thicker shadow masks have the advantage of producing channels with much smaller frosted regions, albeit at the expense of a slightly reduced etch rate. 


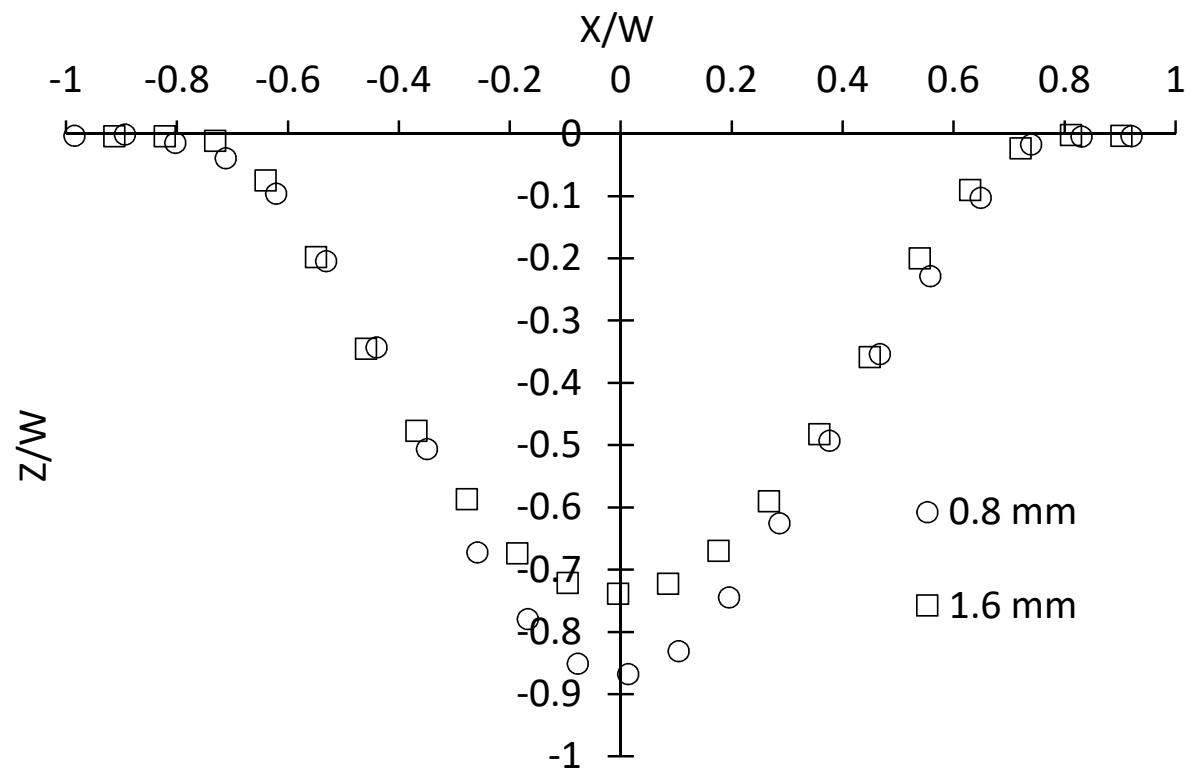

Fig. 2.11 Effect of mask thickness on typical channel profiles obtained after 4 passes of the shadow mask of Fig. 2.2. Channel depth and width normalized by mask opening $W=270 \mu \mathrm{m}$.

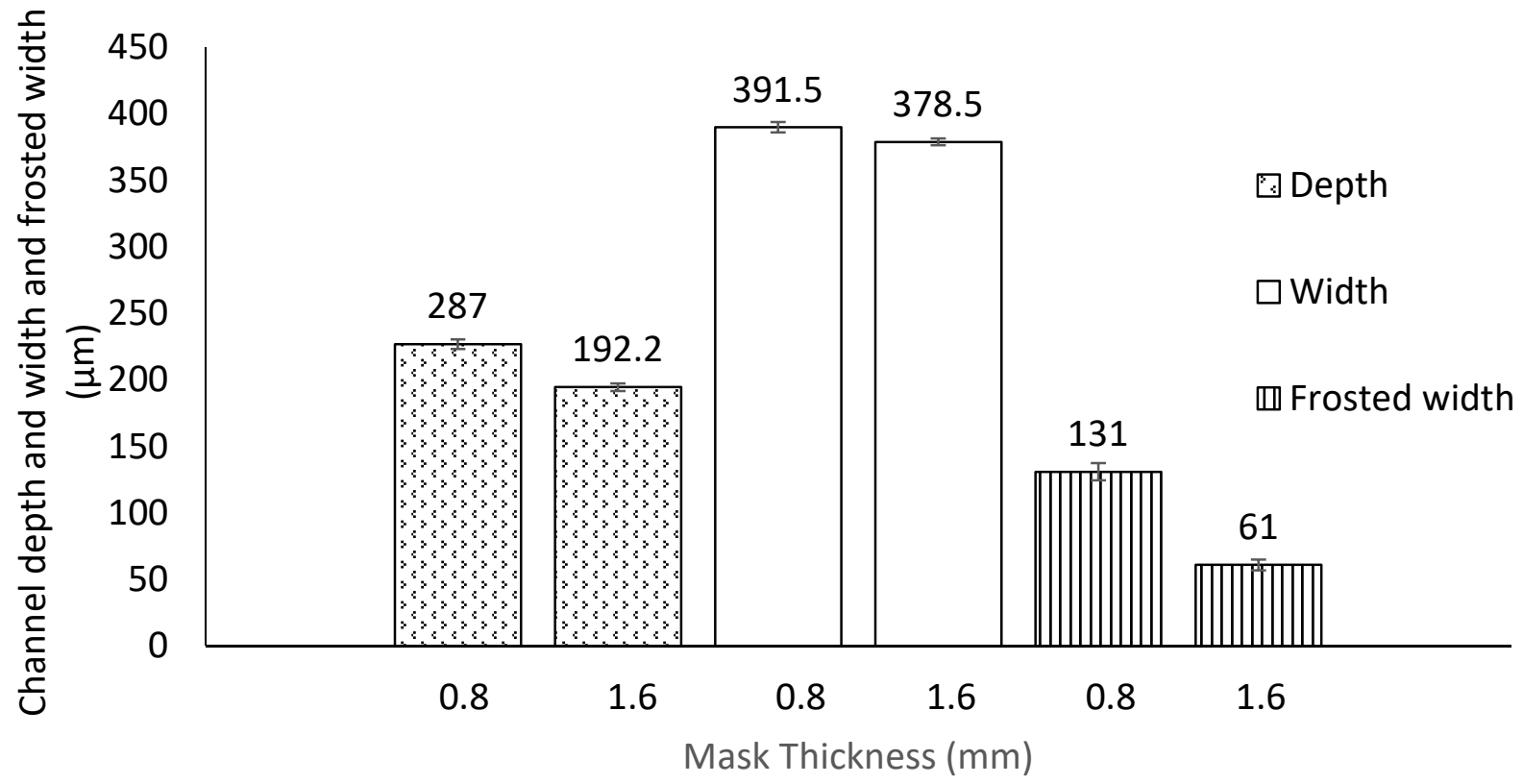

Fig. 2.12 Average channel depth, width and frosted width for mask thicknesses of 0.8 and 1.6 $\mathrm{mm}$. The scatter bars represent \pm 1 standard deviation for 9 measurements ( 3 measurements on each of 3 specimens). 


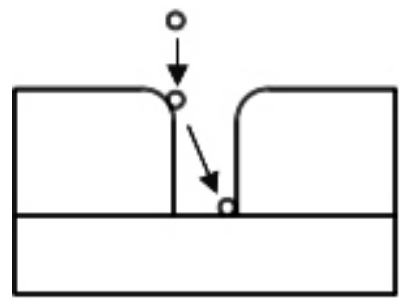

(a)

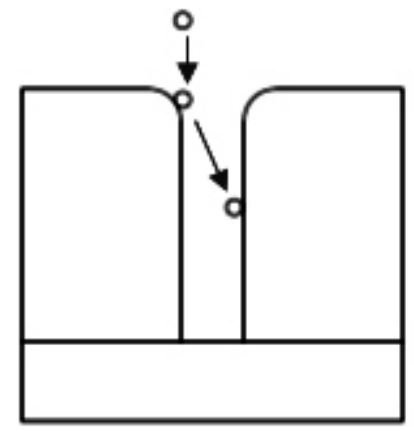

(b)

Fig. 2.13 A particle striking the edge of a thin mask (a) is more likely to strike the surface, while that striking a thick mask (b) collides with the opposing mask.

\subsubsection{Mask standoff}

The effect of a $2 \mathrm{~mm}$ shadow mask standoff was investigated using the shadow mask of Fig. 2.2 with a mask opening of $270 \mu \mathrm{m}$. Fig. 2.14 and Fig. 2.15 show that introducing a $2 \mathrm{~mm}$ gap between the mask and the glass resulted in statistically significant changes (t-test, $\mathrm{p}<0.001$ ) in the depth (20\% decrease), width (7\% increase), and frosted zone width ( $70 \%$ increase) of the channels. The decrease in depth was mainly due to a reduction in the surface flux due to increased jet spreading and a decrease in the particle velocity due to drag. When the mask was not in contact with the surface, particles were decelerated quickly by the drag of the relatively quiescent air encountered after passing through the shadow mask opening. Using a modification of Li's model [48], it was found that this additional drag decreased the particle velocity from approximately 159 $\mathrm{m} / \mathrm{s}$ to $143 \mathrm{~m} / \mathrm{s}$. Assuming that the erosion, $E$, depends on the normal component of particle velocity, $V_{n}$, raised to an exponent of $K=1.43$ for glass with $\mathrm{Al}_{2} \mathrm{O}_{3}$ particles, where Ghobeity et al. [9] presented its equation as $E=C V_{n}{ }^{1.43}$, where $C$ is a constant, and letting all other experimental conditions such as mass flux and particle size be the same, the predicted decrease in depth due to this velocity decrease was $14 \%$. This is in fair agreement with the actual decrease of $19 \%$, particularly since this estimate does not consider the additional effect of the decrease in surface flux over the $2 \mathrm{~mm}$ standoff. 
The increase to a $2 \mathrm{~mm}$ mask standoff also allowed particles ricocheting from the mask edge to strike the target surface beneath the mask, thereby explaining the observed widening of the channel and the larger frosted zones.

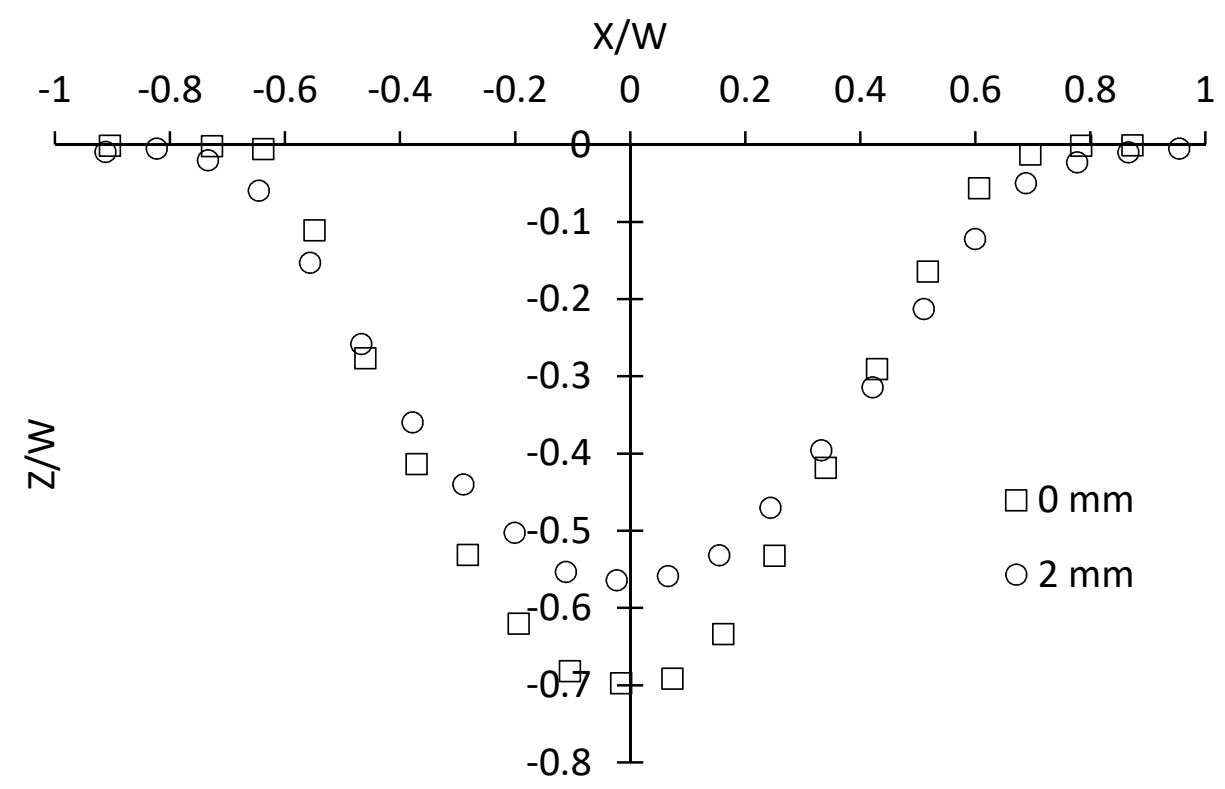

Fig. 2.14 Typical channel profiles obtained after 4 passes of the shadow mask of Fig. 2.2 using mask-to-surface standoff distances of 0 and $2 \mathrm{~mm}$ (Fig. 2.1). Channel depth and width normalized by mask opening $W=270 \mu \mathrm{m}$. 


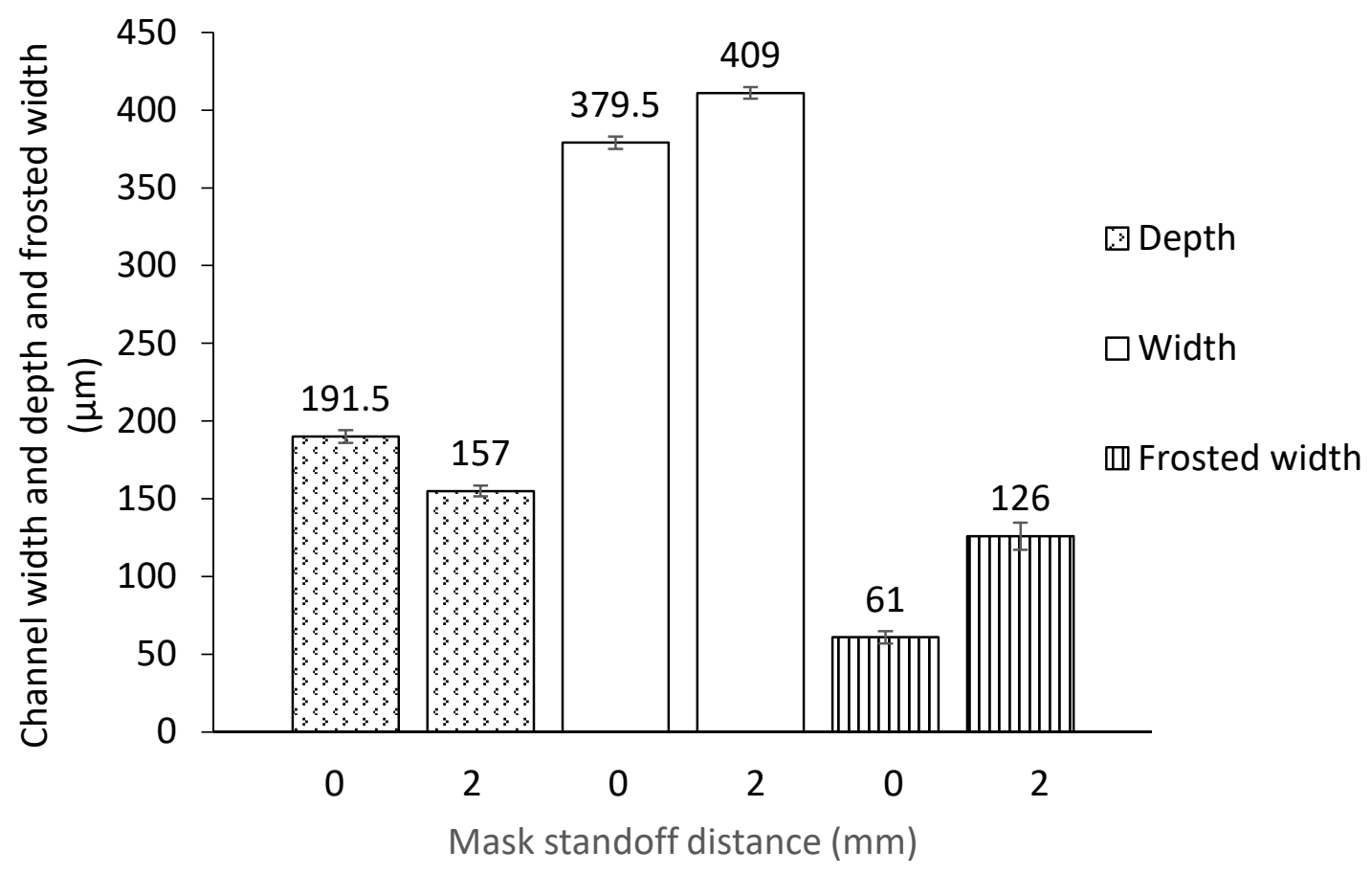

Fig. 2.15 Average depth, width and frosted width for mask standoffs of 0 and $2 \mathrm{~mm}$. The scatter bars represent \pm 1 standard deviation for 9 measurements ( 3 measurements on each of 3 specimens).

\subsubsection{Effect of particle size}

The effect of particle size was studied using 10 and $25 \mu \mathrm{m}$ alumina powder blasted at 200 $\mathrm{kPa}$ through a contacting shadow mask of the type shown in Fig. 2.2 with an opening of $290 \mu \mathrm{m}$. This produced very similar mass flow rates of 4.49 and $4.7 \mathrm{~g} / \mathrm{min}$ for the 10 and $25 \mu \mathrm{m}$ powder, respectively, and therefore the erodent doses delivered to the surface were nearly the same for both particle sizes. Increasing the particle size resulted in deeper and narrower channels, as shown in Fig. 2.16 and Fig. 2.17. Increasing the particle size from 10 to $25 \mu \mathrm{m}$ increased the depth by $25 \%$ and decreased the width by $8 \%$, both of which were statistically significant changes (t-test, $\mathrm{p}<$ 0.005). Recalling that the mass flow rates were nearly identical for both particle sizes, the increase in depth was mostly due to the greater velocity of the $25 \mu \mathrm{m}$ particles $(159 \mathrm{~m} / \mathrm{s}$, using the model presented by Li et al. [48]) compared to the $10 \mu \mathrm{m}$ particles $(141 \mathrm{~m} / \mathrm{s})$. Using the same analysis as in Section 3.4 (i.e, assuming that the erosion, $E$, depends on the normal component of particle velocity, $V_{n}$, raised to an exponent of $K=1.43$ for glass) with both sizes of particles, and considering 
the slight difference in mass flow rates noted above, the predicted increase in depth due mainly to this velocity increase was $24 \%$, which agrees well with the measured value of $25 \%$.

The increase in channel width with decreasing particle size is a well-known phenomenon and was presented by Ghobeity et al. [29], which is due to the fact that smaller particles can pass closer to the mask edge without striking the mask than can larger particles.

There was a near doubling of the frosted width when the particle size was increased from 10 to $25 \mu \mathrm{m}$ (Fig. 2.17). As explained previously, since the shadow mask was sliding on the target, erodent particles became trapped under the mask and raised it slightly. This elevation was greater for $25 \mu \mathrm{m}$ particles than for $10 \mu \mathrm{m}$ particles, and consequently more of the smaller particles in the distribution were able to reach beneath the mask and produce frosting.

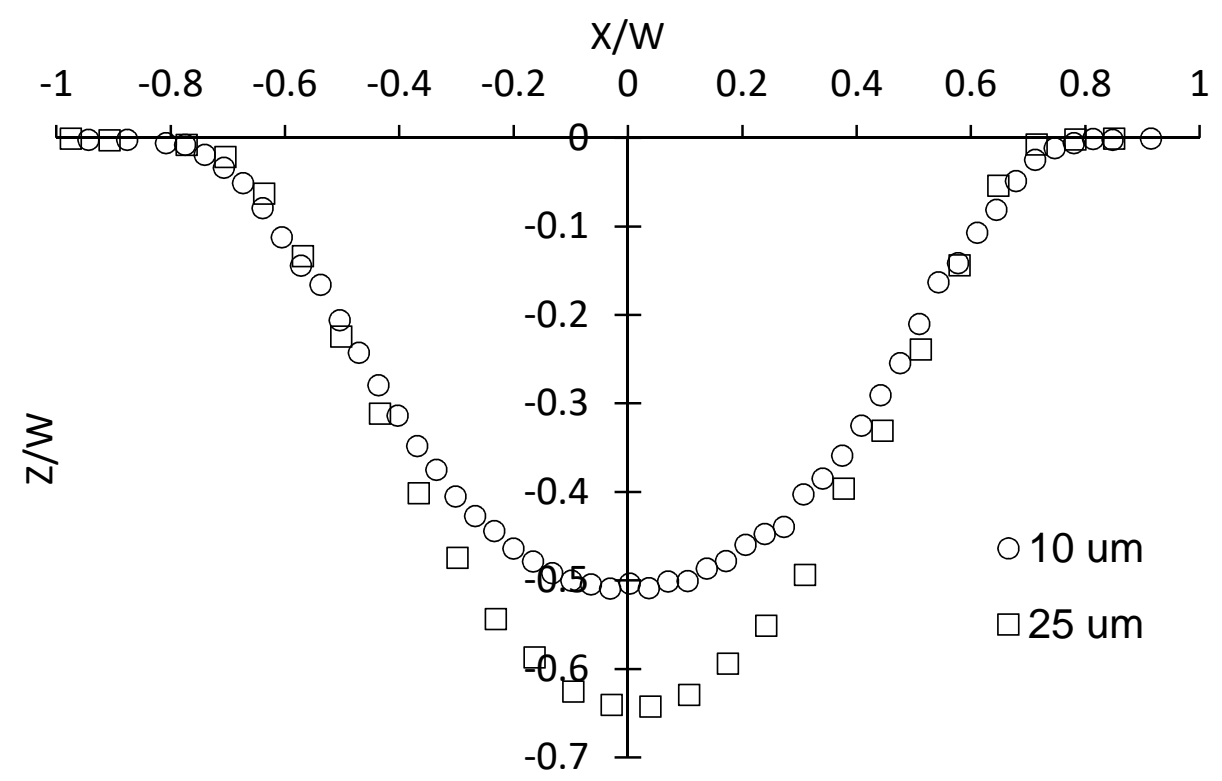

Fig. 2.16 Channel profiles obtained after 4 passes of the shadow mask of Fig. 2.2 using 10 and $25 \mu \mathrm{m}$ particles. Channel depth and width normalized by mask opening $W=290 \mu \mathrm{m}$. 


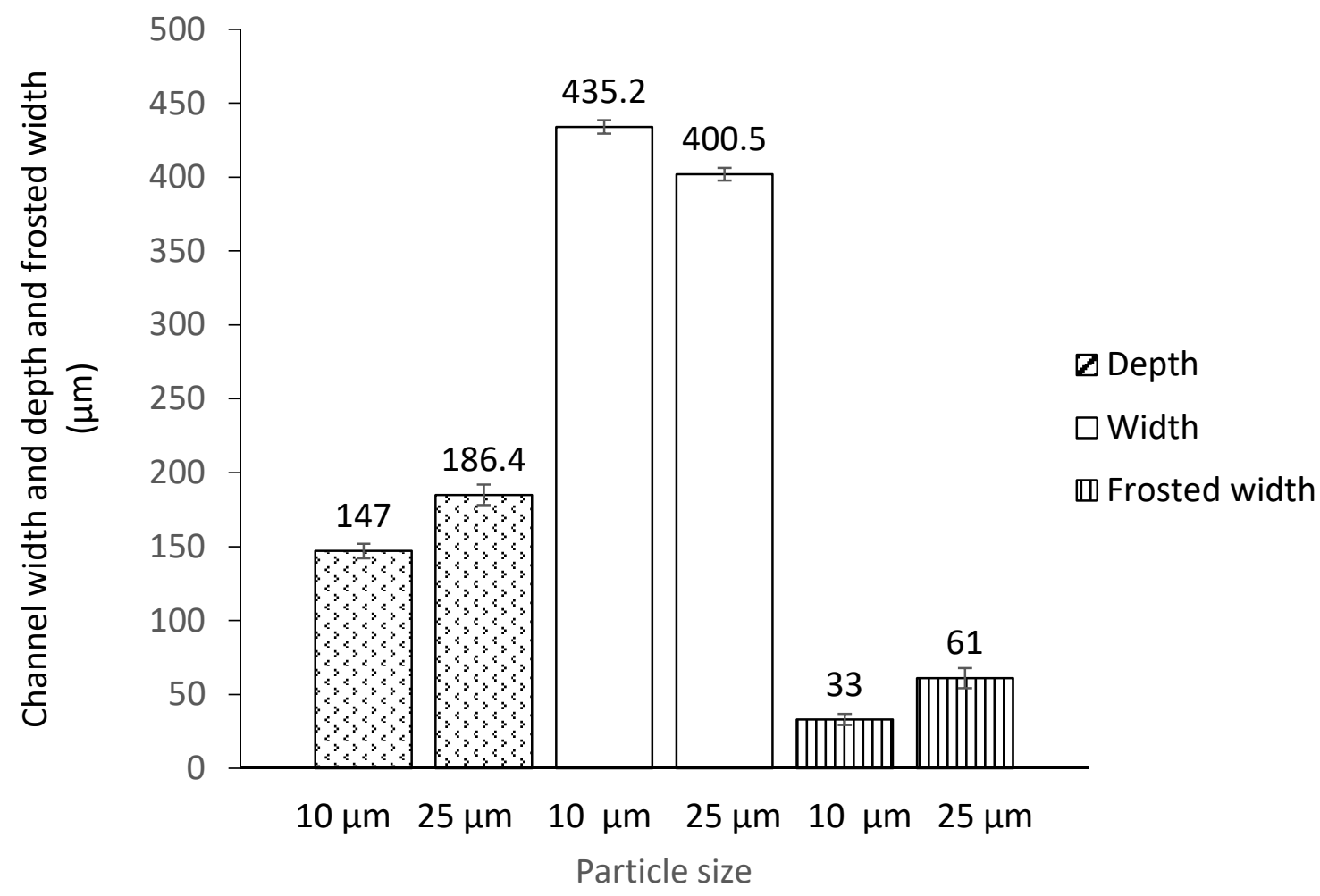

Fig. 2.17 Average channel depth, width and frosted width for particle sizes of 10 and $25 \mu \mathrm{m}$. The scatter bars represent \pm 1 standard deviation for 9 measurements ( 3 measurements on each of 3 specimens).

\subsubsection{Crossed shadow masks}

The shadow mask of Fig. 2.2 produced a narrow impacting erodent jet, but with a fanshaped spread in its scanning direction due to the plume divergence in the narrow direction of the $0.3 \times 3.8 \mathrm{~mm}$ rectangular nozzle. This restricted the arrangement of Fig. 2.2 to the machining of straight channels as shown. In order to achieve a more compact abrasive jet that was narrower in all directions - one with greater potential to be used as a direct write abrasive jet micro-machining technology - a crossed arrangement of two mutually perpendicular sets of shadow masks was utilized (Fig. 2.3).

Typical channel profiles made under identical conditions using crossed and uncrossed contacting shadow masks are shown in Fig. 2.18. The average depth using the crossed mask was found to be $9 \%$ smaller than for the uncrossed mask (average of 3 measurements on each of 3 channels). This difference was found to be statistically significant (t-test, $\mathrm{p}<0.005)$. The channel 
widths for the two arrangements were, however, essentially the same; i.e. a $4 \%$ difference which was statistically insignificant (t-test, $\mathrm{p}>0.25$ ).

The decrease in the channel depth was likely due to the reduction in particle velocity with the crossed mask because of the increased effective mask standoff from the top set of masks to the surface. In addition, the crossed mask reduced the total particle dose delivered to the surface from the much smaller opening.

As shown in Fig. 2.19, utilizing the crossed mask decreased the length of the jet footprint, thereby reducing the particle dose in the direction of scanning. This led to a reduced frosted zone size compared to the regular shadow mask since fewer particles were available to leak between the lower set of masks and the surface. The average width of the frosted zone using the crossed mask was the lowest of all experiments, approximately $30 \mu \mathrm{m}$, which $50 \%$ less than the frosted width for a regular shadow mask $(61 \mu \mathrm{m})$. The asymmetry in the crossed-mask footprint in Fig. 2.19 occurred because of the air gap, equal to the thickness of the bottom masks, that was introduced between the top set of masks and the surface, which allowed some spreading of particles in the direction of the bottom slot (Fig. 2.3).

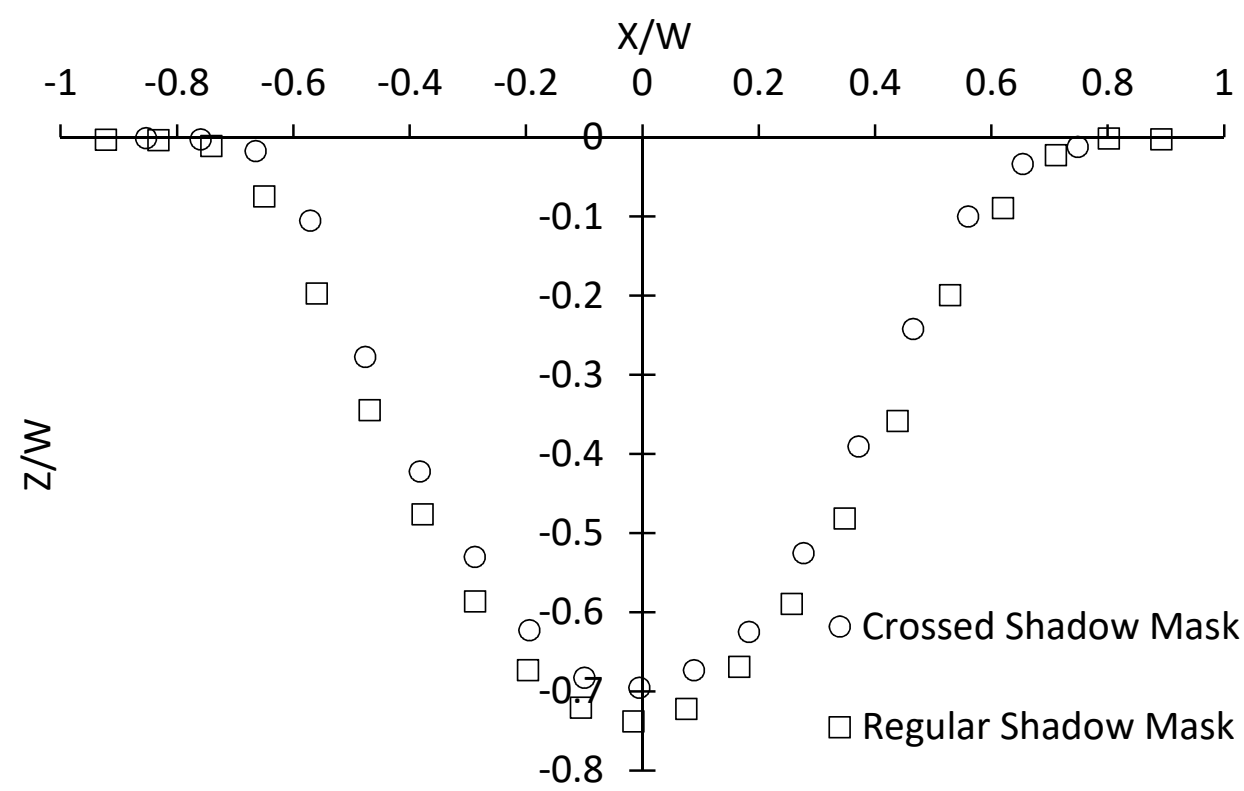

Fig. 2.18 Profiles of channels made after 4 passes of the crossed shadow mask (Fig. 2.3) and the regular shadow mask (Fig. 2.2). Channel depth and width normalized by mask opening $W=290$ $\mu \mathrm{m}$. 


\section{$1000 \mu \mathrm{m}$

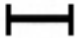

Fig. 2.19 Footprints of the erodent jets produced using the crossed shadow mask (left) and the regular mask (right). For the crossed mask, the slot of the top set of masks was in the vertical direction.

Fig. 2.20 shows an example of the direct writing ability of this technique for the machining of complex curved patterns. The width and depth of the lines in the machined logo are approximately, $700 \mu \mathrm{m}$ and $90 \mu \mathrm{m}$ respectively.

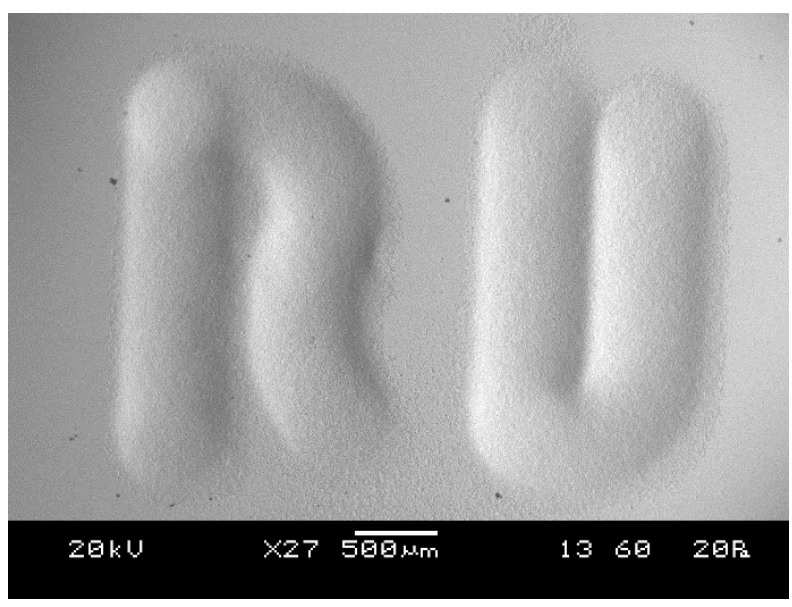

Fig. 2.20 Ryerson University logo machined in glass using direct writing technique with crossed shadow masks.

\subsection{Summary}

A novel adjustable shadow mask has been developed consisting of two parallel metal strips attached to a nozzle for the abrasive jet micro-machining of straight channels. The shadow mask eliminates the need for costly and time consuming mask fabrication and attachment to the 
substrate, and allows for the straightforward adjustment of the mask opening. Compared to a traditional mask attached to the surface, the micro-channels machined using the shadow mask were found to be wider, and were bounded by a frosted regions adjacent to the channel edges. The effects of nozzle standoff, mask standoff, particle size and mask thickness on the channel width, depth and size of the frosted region were investigated and could be explained in terms of changes in the particle velocity and impact location. Increasing nozzle standoff and mask thickness, and decreasing mask standoff and particle size led to the narrowest features with the least amount of frosting. Taking this into account, it was possible to machine micro-channels that were only approximately $10 \%$ wider than those machined using traditional masks up to an aspect ratio of approximately 0.5 . Under these conditions, the frosted regions appeared in bands approximately $60 \mu \mathrm{m}$ wide. By using two sets of crossed masks, the channel width remained unchanged, but it was found that the frosted zone size decreased by 50\% (30 $\mu$ m wide bands). It was demonstrated that this crossed-mask configuration had the additional advantage that it could also be used to direct write curved features.

Because the masks are attached to the nozzle, it was thought that the shadow mask methodology would be appropriate for machining helical micro-channels using AJM on a lathe that rotates a glass or PMMA rod. However, because of the intricacy of synchronizing the rotational and axial feed speed of the lathe, helical extension springs were preferred to this apparatus (Chapter 5). However, if a synchronization technique can be employed, the shadow mask can be exploited in future work. 


\section{Chapter 3 Abrasive jet machining of channels on highly-curved glass and PMMA surfaces}

This chapter is based on the following published paper:

A. Nouhi, K. Kowsari, J.K. Spelt, M. Papini, "Abrasive jet machining of channels on highlycurved glass and PMMA surfaces”, Wear, vol. 356-357, pp. 30-39, 2016.

\subsection{Introduction}

The micro-machining of small curved specimens made of brittle materials such as glass and ceramics and ductile materials like polymers and metals is required in optical and biomedical equipment [1]. Martinez and Chalkof [50] reviewed different strategies of fabricating metal or polymer stents, tubular supports placed temporarily inside a blood vessel, canal, or duct by micropatterning their surfaces in order to aid healing or relieve an obstruction.

Li et al. [33] studied the radial-mode abrasive waterjet turning process on $59 \mathrm{~mm}$ diameter steel rods. They concluded that the machined depth increased as the jet incidence approached the surface normal, and when a higher water pressure, a higher surface speed, or a lower feed rate were used. Manu and Babu [35] implemented Finnie's theory of erosion to model the material removal from a $25.4 \mathrm{~mm}$ rotating cylinder using abrasive waterjet turning, considering the continuous change in the local impact angle caused by the reduction in workpiece diameter. The nozzle had an initial radial offset relative to the rod so that the erosion took place at the periphery of work piece.

Abrasive jet micro-machining (AJM) has the ability to cut and mill many materials without excessive forces or thermal damage, which is a significant advantage over many other machining technologies [1]. In addition, AJM does not involve hazardous materials or equipment, does not require a clean room, and has a low capital and operating cost [51]. Target material is removed by the impact of a jet of small high-speed particles producing either brittle or ductile erosion [4]. The erosion rate in brittle materials is characterized by a peak at normal incidence, while the erosion rate of ductile materials has a peak at an oblique impact angle. As a result, the shape of relatively deep features micro-machined in brittle and ductile materials under identical conditions is different. For example, the dependency of the erosion rate on the local sidewall slope causes AJM 
channels machined in ductile materials to have much steeper sidewalls and flatter bottom sections than those in glass channels [6].

AJM has found use primarily in the machining of initially flat surfaces at perpendicular incidence. Slikkerveer and in't Veld [22] and ten Thije Boonkamp and Jansen [21] introduced the first analytical AJM models for predicting the channel shapes machined on brittle flat surfaces. Ghobeity et al. [9] modified the model of [21] to include the erosive characteristics of the abrasive jet as determined by the cross-sectional profile of a shallow calibration channel or by a fundamental equation giving the variation of the particle flux and speed across the jet (Fig. 3.1a). Kong et al. [52] also used measured shallow calibration profiles to characterize the erosive 'footprint' for abrasive water jet machining (AWJM). Once characterized in this manner, they presented an analysis that allowed for the AWJM jet footprint to be predicted for any jet feed speed and jet path. The model of [9] was used to predict masked and unmasked channel profiles machined in brittle materials. Since the model was developed for brittle materials, only the normal component of particle velocity to the target surface was considered. The plume of an abrasive jet is approximately conical, introducing a spread in particle trajectories and a particle flux that decreases with increasing nozzle to surface (standoff) distance. In these studies, the effects of the spread in particle trajectories on the local particle impact angles and standoff distances were neglected, i.e. the trajectories were all assumed parallel to the jet axis at a single standoff, because the divergence angle was only on the order of $10^{\circ}$ or less. Although this introduced errors in the prediction of the surface evolution when the channels became deeper and developed steep side walls, the errors are much smaller than they would be on a curved surface, where even when uneroded, the surface curvature can introduce a much wider range of impact angles and standoff distances. 
a

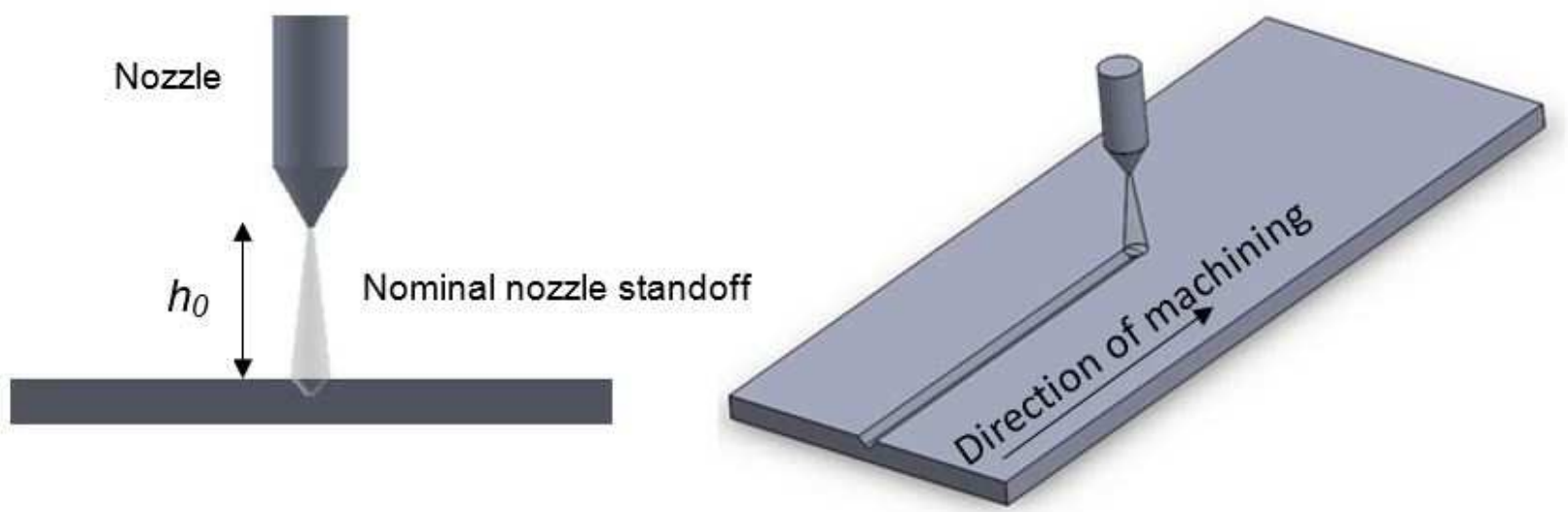

b

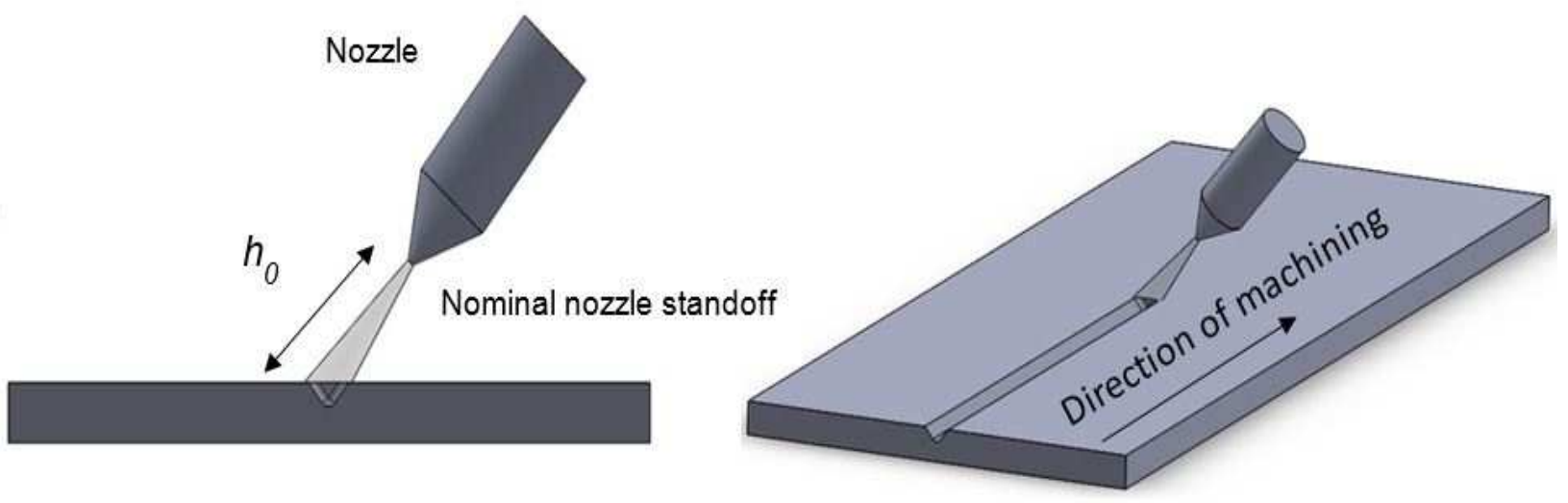




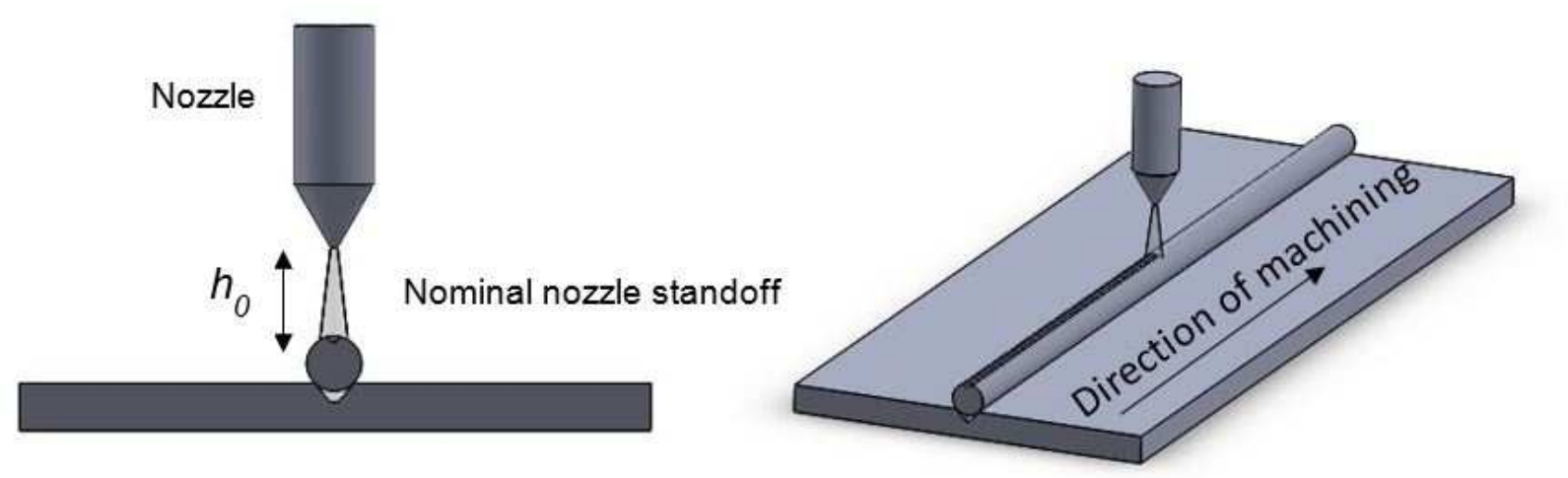

Fig. 3.1 Schematic of channel machining processes. (a) Channel made at normal impact angle on flat target, (b) Channel made at oblique impact angle on flat target, (c) Channel made axially on rod. Conical divergence of particle jet causes local standoff and impact angle to vary across footprint in parts (b) and (c).

Getu et al. [6] extended the method of [9] by considering the impact angle dependency of erosion rate to predict the cross-sectional profiles of unmasked and masked channels made in flat ductile materials such as polymethylmethacrylate, in which both the normal and tangential velocity components affect erosion. These models also neglected the effect of jet divergence and local standoff, and the greater sensitivity of ductile materials to impact angle likely resulted in the observed under-prediction in width and over-prediction of channel depth.

Ally et al. [30] subsequently implemented the surface evolution equation of [6] to predict the shapes of unmasked channel profiles machined in flat metal targets at oblique incidence as in Fig. 3.1b. The velocity and mass flux distributions across the jet were inferred by curve fitting to a shallow channel machined at normal incidence (Fig. 3.1a) and then transformed to obtain the erosive efficacy on a tilted target. It was observed that the model resulted in an overestimation of the channel width, which, as will be shown in Section 3.4.3, could be attributed to the neglect of the greater effect of jet divergence on the local impact angles and standoff. 
Burzynski and Papini [31] developed a level-set model (LSM) to predict the surface evolution of high aspect-ratio masked and unmasked channels machined into flat brittle targets at normal (Fig. 3.1a) and oblique (Fig. 3.1b) incidence, and considering particle second strikes. This model accounted for the change in the standoff distance and spread in particle trajectories which affected the local flux for very deep channels. In the case of tilted channels, it was found that it was convenient to move the origin of the coordinate system from the target surface to the nozzle exit. This simplified the formulation since the complex transformations of particle trajectories was made unnecessary. The predictions of the model were generally in good agreement with experiments, and the model was able to shed light on the role of second strike and other complex phenomena when eroding high aspect ratio features onto flat surfaces. However, as will be discussed below, such LSM formulations are too complex to routinely implement.

AJM has a largely unexplored potential for machining tilted and curved surfaces, such as found in the turning of small cylindrical parts, the micro-patterning of rods, and the production of threads on materials such as glass and ceramics which are otherwise difficult to machine [1]. Consequently, the AJM of highly-curved surfaces has received very little attention. A notable exception is the work of Kim et al. [53] who designed and fabricated masks suitable for machining arbitrarily curved surfaces.

As will become apparent, the application of existing analytical AJM surface profile models to the machining of highly-curved target surfaces accentuates the errors due to the neglect of jet divergence and local variations in the standoff distance. As mentioned above, the LSM models of Burzynski and Papini [31] consider both of these effects, but these were implemented in an LSM formulation for channels machined in flat targets. Compared to traditional surface evolution models, the LSM formulations are much more complex, and the numerical grid must be modified and hard-coded for each new target geometry. The present work aims to establish a new surface evolution model in the form of a single partial differential equation that can be solved using standard methods to allow the prediction of the cross-sectional shapes of channels made at arbitrary incidence on virtually any target geometry by considering the local variations of incident particle trajectory and standoff distance across the jet footprint. The performance of the model was evaluated by comparison with experiments for the case of channels machined on highly curved brittle (glass) and ductile (PMMA) targets. 


\subsection{Surface profile modelling}

\subsubsection{Background}

The surface evolution model to predict the cross-sectional profiles of channels machined in initially flat brittle materials using AJM, where only normal component of particle velocity is relevant, was presented in [9] as

$$
Z_{,}-E(x)\left(1+Z_{, x^{2}}\right)^{-k / 2}=0
$$

where $Z,_{t}$ and $Z{ }_{x}$ are the partial derivatives of the profile depth $Z$ with respect to time and the coordinate $x$, defining the width dimension (Fig. 3.2a), $E(x)$ is the erosive efficacy distribution applied to the exposed target surface by the passage of the nozzle (i.e. the lateral spatial distribution of the potential of the jet to erode), and $k$ is a velocity exponent related to the erosive characteristics of the surface material. Therefore, $E(x)$ accounts for the erosion generated by the entire jet footprint as it passes a given point.

For ductile materials, which have a more complex dependence of erosion on the local impact angle, the surface evolution model of [6] is given by

$$
Z{ }_{, t}-E(x) \sqrt{1+Z,,_{x}^{2}} g(\alpha)=0
$$

where

$$
g(\alpha)=(\sin \alpha)^{n_{1}}\left(1+H_{v}(1-\sin \alpha)\right)^{n_{2}}
$$

which describes the dependence of the erosion rate on the impact angle, $\alpha$, defined between the incident velocity vector and the local tangent to the surface as 


$$
\alpha=\left(\frac{\pi}{2}-\arccos \left(\frac{1}{\sqrt{1+Z, x^{2}}}\right)\right)
$$

$H_{v}(\mathrm{GPa})$ is the initial target hardness and the constants $n_{l}$ and $n_{2}$ are determined experimentally and depend on the particle hardness and other impact conditions [12]. As discussed in the Introduction, these surface evolution models for brittle and ductile materials provide good predictions of channel cross-sectional shape at perpendicular and oblique incidence for unmasked channels machined in flat surfaces at relatively low aspect ratios up to 0.3 [6], [9], [30]. Under these conditions, the neglect of jet divergence and variations in the local standoff distance were insignificant.

\subsubsection{Generalized surface evolution model - varying standoff distance and jet divergence angle}

The effect of neglecting the variation in local standoff distance and jet divergence, which, in turn, affect the local particle flux and impact angle, can be best demonstrated by considering the AJM of a tilted surface. As discussed in the Introduction, the model of Ally et al. [30] used Eqs. (1) and (2), together with a transformation of the erosive efficacy to a tilted coordinate system, in order to predict the surface evolution due to AJM on tilted brittle and ductile targets as in Fig. 3.1b. Fig. 3.2a illustrates the assumptions of this model for a nozzle angle $\theta=60^{\circ}$; i.e. all particles are assumed to have an impact angle of $60^{\circ}\left(\gamma_{0}=30^{\circ}\right)$ and a uniform standoff distance of $h_{0}=10 \mathrm{~mm}$. However, as shown in Fig. 3.2b, the local impact angles actually vary from $64^{\circ}$ to $53^{\circ}$ for the 460 $\mu \mathrm{m}$ nozzle used in the present experiments. Moreover, the actual standoff distance varied from 8.5 to $12 \mathrm{~mm}$ for the nominal nozzle standoff distance of $10 \mathrm{~mm}$. Similarly, for the AJM of a $5 \mathrm{~mm}$ rod at nominally perpendicular incidence using the same nozzle at a $10 \mathrm{~mm}$ standoff, the local impact angles vary from $90^{\circ}$ at the centerline (point $f$ in Fig. 3.3) to $\alpha=54^{\circ}$ where the conical plume of the abrasive jet intersects the rod (point $g$ in Fig. 3.3). Ignoring the spread of the jet and considering all the particles to travel parallel to the nozzle axis would give an apparent local impact angle of $\alpha=60^{\circ}$ at point $g$ in Fig. 3.3. The local standoff distance at point $g$ is actually $10.5 \mathrm{~mm}$. 
As will be seen below, these errors in the local impact angle and standoff distance can make the predictions of Eqs. (1) and (2) increasingly inaccurate for highly-curved targets.

In deriving a surface evolution model that takes into account variations in standoff distance and local impact angle for curved targets, two approaches were used, each giving identical results. In the first approach, the coordinate system was attached to the surface as in Fig. 3.2, the erosive efficacy was mapped to the curved or tilted surface using a methodology similar to that by Ally et al. [30], and then the spread in local incident angles (not considered by Ally et al.) and change in local standoff distance were calculated using geometric considerations While this method provided a direct understanding of how the erosive efficacy spread on a curved surface, it was relatively complex and not generally applicable since it required different geometric relations for each target geometry. Instead, a second, more simple generally applicable approach with the coordinate system fixed to the nozzle (Fig. 3.3) will be presented in detail in the following sections. As will be seen, using this method, the target surface geometry appears only in the initial condition of the surface evolution equation.

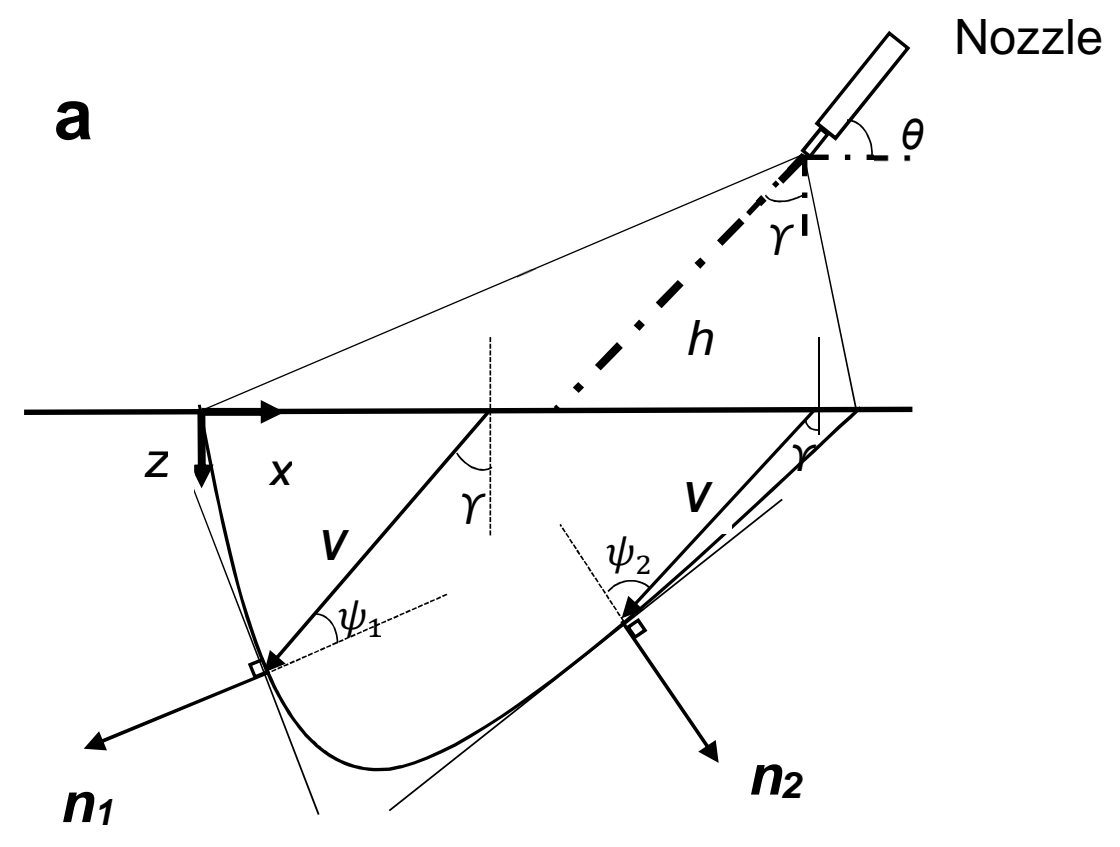




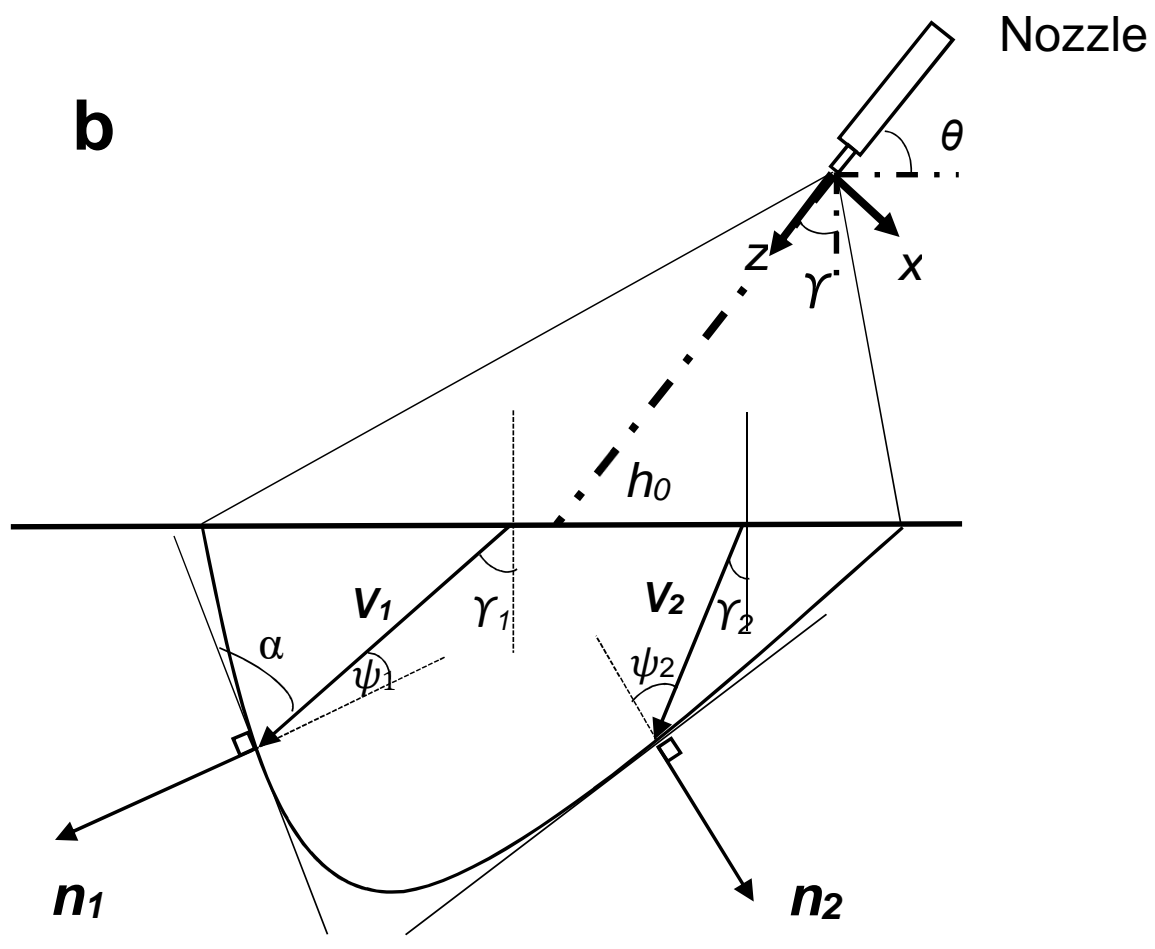

Fig. 3.2 centerline (e.g. $V_{l}$ and $V_{2}$ ) and coordinate system is located on the target, (b) new model where incident angle varies across jet and coordinate system is located at nozzle tip. $n_{1}$ and $n_{2}$ are unit vectors normal to channel profile at point of impact, $\psi_{1}$ and $\psi_{2}$ define local angle between impact velocity vector and outward normal to surface. $h_{0}$ is the nominal standoff distance. 


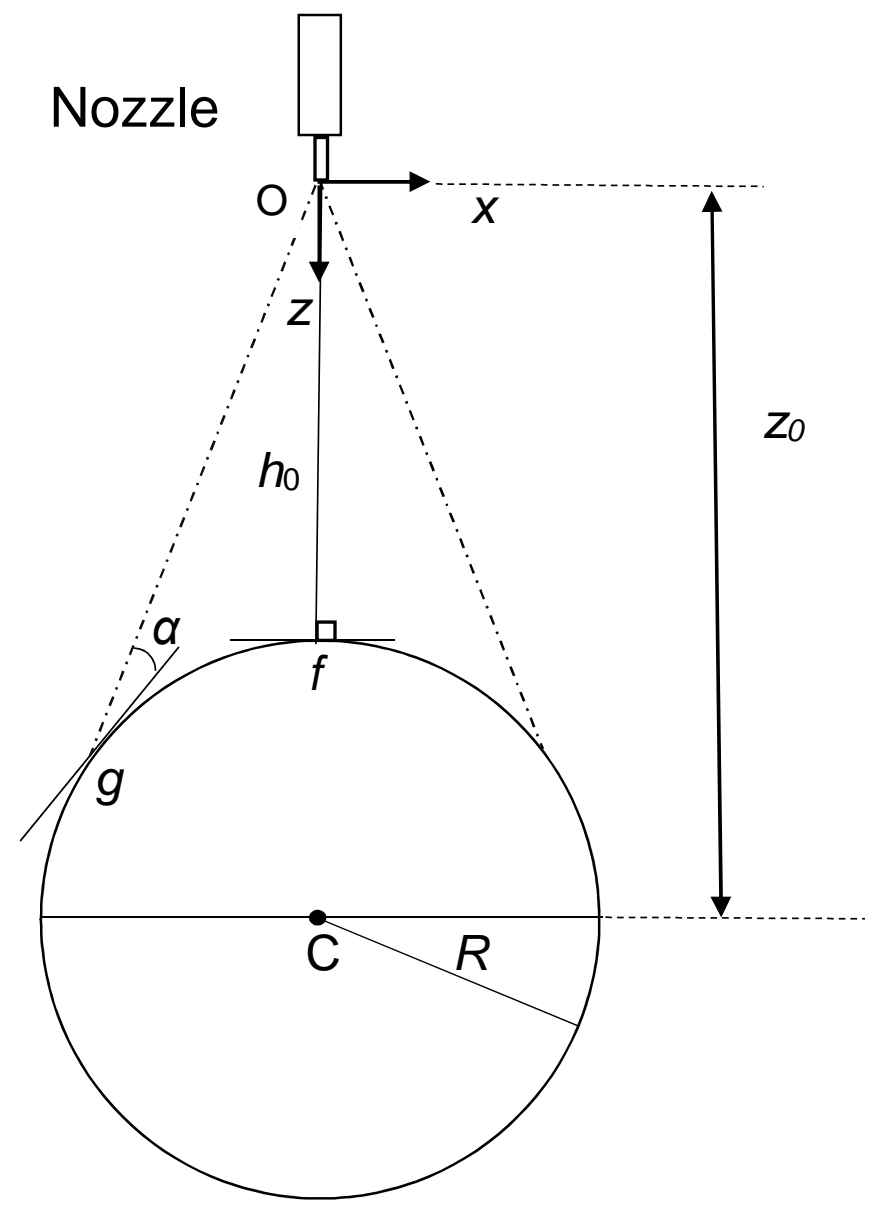

Fig. 3.3 Particle impact on rod at jet centerline $(f)$ and at edge of nozzle footprint $(g)$ illustrating variation in local impact angle, $\alpha$, and standoff due to target curvature and jet divergence.

\subsubsection{Development of generalized model- brittle materials}

Following the original formulation of ten Thije Boonkamp and Jansen [21], the surface evolution model at any location on an arbitrary surface, Fig. 3.2a, is given by:

$$
Z, t-c \sqrt{1+Z_{, x}^{2}}=0
$$

where the velocity of the eroding surface in a local direction normal to the surface is given by, $c$,

$$
c=\frac{1}{\rho_{s}} G \boldsymbol{\Phi} \boldsymbol{n}
$$


where $\rho_{s}$ is the density of the target, $G$ the specific erosion rate, defined as the ratio of the mass loss of the substrate to the mass of erodent used, and $\boldsymbol{\Phi}$ is the particle mass flux in the direction of the particle velocity vector, $\boldsymbol{V}$, (Fig. 3.2a and Fig. 3.2b). The specific erosion rate, $G$, can be modeled as [9],

$$
G=C(|\boldsymbol{V}| \cos \psi)^{k}
$$

in which $C$ is an erosion constant, $\psi$ is the angle between $\boldsymbol{V}$ and the outward normal to the surface at the point of impact (Fig. 3.2a and Fig. 3.2b), and $k$ is the velocity exponent.

Equations (3.5)-(3.7) have usually been implemented with the coordinate system attached to the target surface (Fig. 3.2a), but are valid for any coordinate system in the plane of Fig. 3.2 provided $\boldsymbol{n}, \boldsymbol{V}$ and $\boldsymbol{\Phi}$ are all expressed with respect to that system. Since the variation of $\boldsymbol{V}$ and $\Phi$ are most easily obtained along and across the jet axis, in the present case where the nozzle may be tilted or the surface has significant curvature, it is more convenient to attach the coordinate system to the nozzle as shown in Fig. 3.2b. With this in mind, the outward unit vector normal to the channel profile, $\boldsymbol{n}$, is given by,

$$
\boldsymbol{n}=\frac{1}{\sqrt{1+Z,,^{2}}}\left(\begin{array}{c}
-Z, x \\
1
\end{array}\right)
$$

and the particle velocity unit vector (Figs. 2 b and 3 ), is

$$
\boldsymbol{e}_{v}=\frac{\boldsymbol{V}}{|\boldsymbol{V}|}=\left(\begin{array}{l}
\sin \gamma \\
\cos \gamma
\end{array}\right)
$$

and

$$
\cos \psi=n \cdot e_{v}=\frac{\cos \gamma-Z,_{x} \sin \gamma}{\sqrt{1+Z,_{x}^{2}}}
$$


where $\gamma$ is the particle trajectory angle which varies across the channel width as

$$
\begin{aligned}
& \cos \gamma=\frac{Z}{\sqrt{x^{2}+Z^{2}}} \\
& \sin \gamma=\frac{x}{\sqrt{x^{2}+Z^{2}}}
\end{aligned}
$$

Combining Eqs. (3.10) and (3.11) gives

$$
\cos \psi=\frac{Z-x Z_{, x}}{\sqrt{x^{2}+Z^{2}} \sqrt{1+Z,_{x}^{2}}}
$$

Combining Eqs. (3.5)-(3.8) with (3.12), leads to the following new, generalized surface evolution equation for oblique or normal incidence machining of a jet on an arbitrarily-shaped brittle material, which takes into account the changes in local impact angles due to jet divergence, and changes in local standoff distance:

$$
Z_{,_{t}}-E(x, Z)\left(1+Z,_{x}{ }^{2}\right)^{-k / 2}\left(\frac{Z-x Z_{x}}{\sqrt{x^{2}+Z^{2}}}\right)^{k+1}=0
$$

where the $\mathrm{x}$ coordinate is attached to the nozzle as in Fig. 3.2b. $Z(x, t)$ is the $z$-location (origin at nozzle as in Fig. 3.2b) of the eroded profile at any time $t$ and $E(x, Z)$ will be defined in Section 3.2.3. This equation describes the cross-sectional profile of a symmetric or asymmetric channel machined in an arbitrarily-shaped brittle target after the passage of the jet. 


\subsubsection{Ductile materials}

To determine the surface evolution model for ductile materials at oblique or normal incidence taking into account jet divergence and changes in the local standoff distance, the local impact angle, $\alpha$ in Eq. (3.4) needs to be modified using Eq. (3.12). This leads to

$$
\alpha=\left(\frac{\pi}{2}-\cos ^{-1}\left(\frac{Z-x Z,_{x}}{\sqrt{x^{2}+Z^{2}} \sqrt{1+Z_{,_{x}}^{2}}}\right)\right)
$$

which can be substituted into Eq. (3.2) to yield

$$
Z, t-E(x, Z) \sqrt{1+Z_{, x}{ }^{2}} g\left(\frac{\pi}{2}-\cos ^{-1}\left(\frac{Z-x Z_{, x}}{\sqrt{x^{2}+Z^{2}} \sqrt{1+Z_{, x}^{2}}}\right)\right)=0
$$

where $x$ is again in terms of the coordinate system attached to the nozzle and $Z(x, t)$ is the $z$-location (origin at nozzle as in Fig. 3.2b) of the eroded profile at any time $t$ and. Equations (3.13) and (3.15) require the erosive efficacy incident to the surface, $E(x, Z)$, referred to this coordinate system. The determination of the erosive efficacy is discussed in the following section.

\subsubsection{Determination of erosive efficacy}

The erosive efficacy of AJM nozzles eroding effectively flat targets was previously determined either by fitting a function $E(x)$ to the measured cross-sectional profile of a shallow (i.e. with negligible slope) channel machined at a single standoff distance or by multiplying the measured velocity and mass flux distributions, $E(x)=\phi(x)|\boldsymbol{V}(x)|^{k}$, in which a Weibull function represented the particle mass flux distribution, and the particle velocity was linearly distributed across the abrasive jet [9]. An approach similar to the latter was adopted in the present work, and in order to facilitate the inclusion of standoff distance as a variable in the present formulation, the function $E(x, Z)$ was based on the measurement of the cross-sectional profile of shallow channels 
at perpendicular incidence at three different standoff distances. This gave the erosive efficacy throughout the conical erosive jet plume as a function of the radial and axial coordinates.

For the nozzle used in the present experiments, the particle velocity distribution was unknown, and therefore assumed constant across the round jet. This assumption, which will be validated by the good fits of erosive efficacy at different standoffs obtained in Section 3.4.1, implies that the variation in mass flux across the jet had a much greater effect on the erosive efficacy than the variation in velocity. Indeed, some evidence for this is shown by the much lower variation of velocity than flux variation for other abrasive jet nozzles in Ref. [54]. The mass flux was assumed to follow a Weibull distribution of both the radial coordinate and the local standoff, $Z, E(x, Z)$ :

$$
E(x, Z)=\delta\left(\frac{\beta}{Z}\right)^{2} e^{-\left(\frac{\beta x}{Z}\right)^{2}}
$$

where

$$
\delta=\left(\frac{C}{\rho_{s}} \frac{\dot{M}}{\pi}\left(|\boldsymbol{V}|^{k}\right)\right)
$$


where $C$ and $k$ are constants related to the erosive characteristics of the surface material of density $\rho_{s}, \dot{M}$ is the mass flow rate, $\beta$ is the nozzle "focus coefficient", with higher values indicating a more focused stream, and $\delta(\beta / Z)^{2}$ is determined by the channel depth. Equation (3.16) corresponds to an erosive efficacy function for a conical jet in which the particles travel in straight lines from the nozzle, Fig. 3.4, i.e. they should be independent of standoff distance. The values were obtained by averaging the $\delta$ and $\beta$ obtained from curve fits to the shallow channel cross-sections at three standoffs.

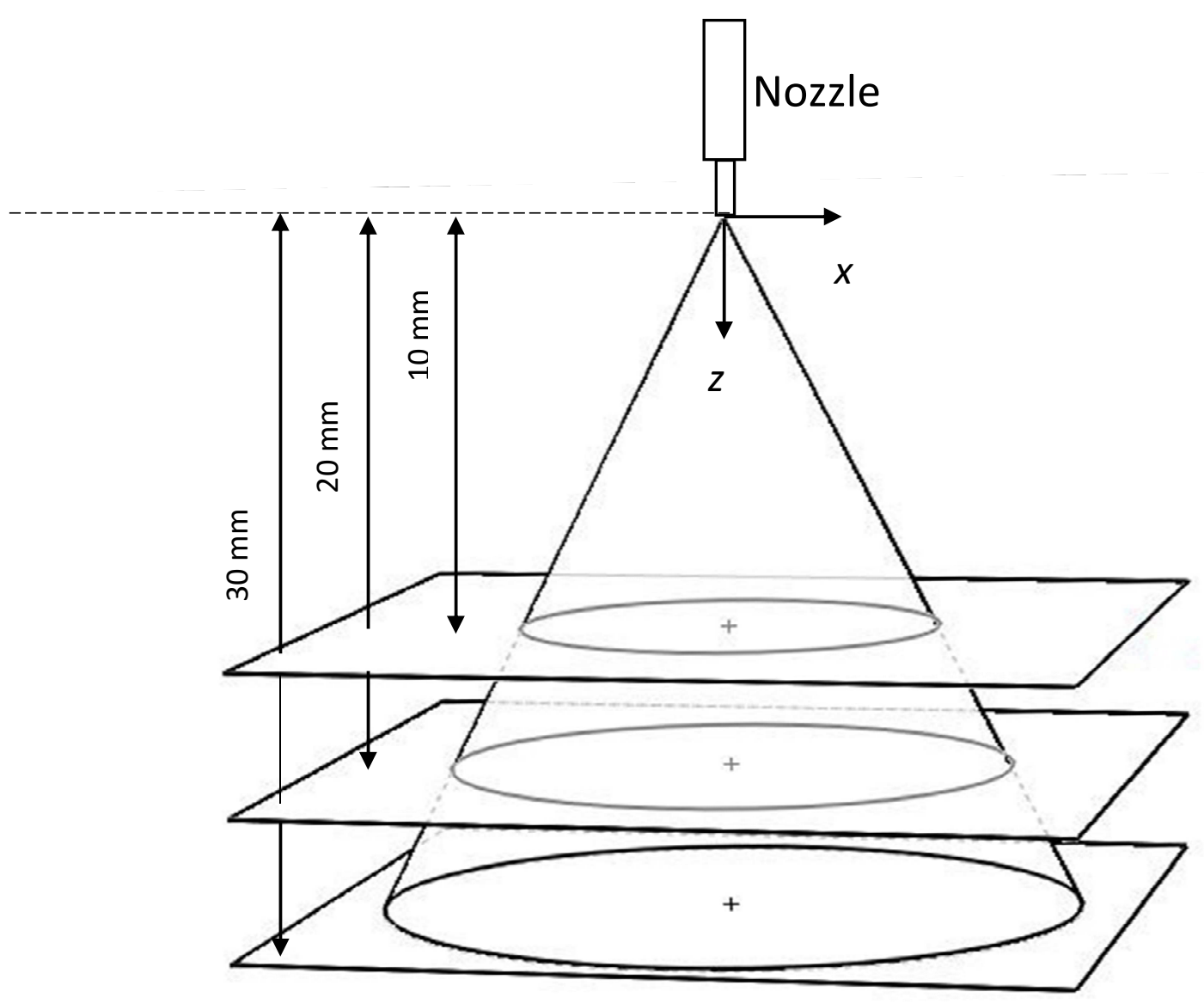

Fig. 3.4 Schematic representation of intersection of abrasive jet plume with successive planes at standoffs of $10 \mathrm{~mm}, 20 \mathrm{~mm}$ and $30 \mathrm{~mm}$, where shallow channels were machined to determine the erosive efficacy function. 
It is noted that, as discussed in [10] for AJM, as long as the scan speed is sufficiently high to result in a relatively small slope at the leading edge of the machining front, the dimensionless shape of the cross-sectional channel profile produced by a scanning AJM nozzle is equivalent to that created across the diameter of a stationary nozzle. In [9] the calculations were performed for the machining of channels on a flat surface. The same calculations for the case of channels machined on rods yielded a maximum error of $7 \%$ between the normalized erosive efficacy of the scanned and stationary nozzle. Therefore, the dimensionless erosive efficacy function obtained from channel profiles is approximately equivalent to that produced using stationary hole profiles.

\subsubsection{Initial conditions}

Once the erosive efficacy was determined, the modified surface evolution equation could be solved with the appropriate initial conditions defined by the target surface geometry as a function of $x$ and $z$, referred to the origin at the nozzle tip.

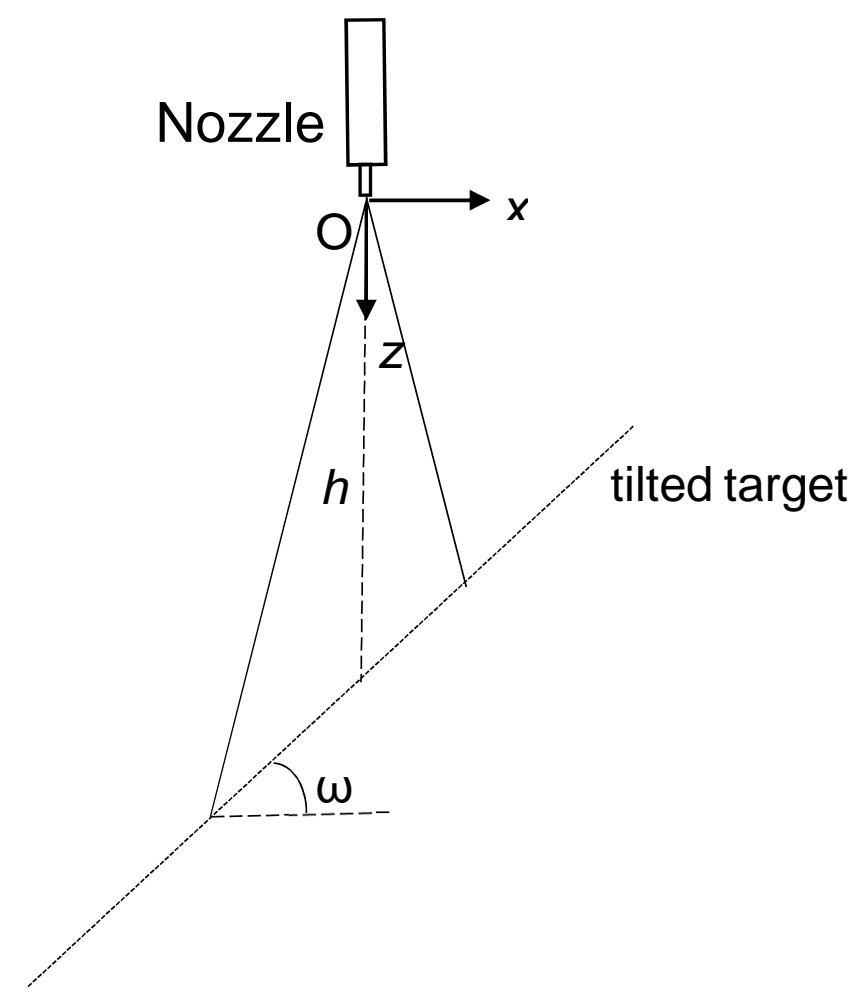

Fig. 3.5 Flat target surface tilted at angle $\omega$. 
The initial condition $(t=0)$ for a flat target tilted at an arbitrary angle $\omega$ (Fig. 3.5) is

$$
Z(x, 0)=-\tan (\omega) x+h_{0}
$$

and for a rod of diameter $R$ (Fig. 3.3)

$$
Z(x, 0)=z_{0}-\sqrt{R^{2}-x^{2}}
$$

The following sections describe the machining of the channel profiles used to evaluate the accuracy of this new surface evolution model for tilted and highly-curved surfaces.

\subsection{Experiments}

\subsubsection{Apparatus}

All experiments were conducted using an AccuFlo AF10 Micro-Abrasive Blaster, (Comco, Inc. Burbank, CA, USA) operating at $200 \mathrm{kPa}$ air pressure. Moisture in the powder reservoir was minimized using a dehumidifier, a refrigeration air dryer, and a desiccant filter on the inlet air. The repeatability of the experiments was enhanced by following the operating procedures described in [45], including the use of a variable-speed rotary electric mixer to avoid powder bed compaction and particle agglomeration inside the reservoir. Aluminum oxide $\left(\mathrm{Al}_{2} \mathrm{O}_{3}\right)$ powder $(\mathrm{Comco}$, Inc. Burbank, CA, USA) with a mean diameter of $11.6 \mu \mathrm{m}$ (log-normal distribution with standard deviation of $3.31 \mu \mathrm{m}$ ) was used in all experiments.

\subsubsection{Channels on flat surfaces}

While the main focus was the machining of highly-curved surfaces, channels were also made on flat surfaces at both normal and oblique incidence to evaluate the accuracy of approaches to account for the variation of the standoff distance and the particle trajectory across the plume. The erosive efficacy function was obtained, as explained in Section 3.2.3 from shallow channels machined in $100 \times 50 \times 3 \mathrm{~mm}$ thick glass (Borofloat, Swift Glass Co. Inc., Elmira, NY, USA) and 
PMMA (type ACRYLITE® FF, CYRO Industries, Rockaway, NJ, USA) samples using a single pass of a $460 \mu \mathrm{m}$ inner-diameter nozzle at standoff distances of 10, 15 and $20 \mathrm{~mm}$ measured along the nozzle centerline (Fig. 3.1a). The nozzle was stationary at $\theta=90^{\circ}$ while the target was moved at $0.5 \mathrm{~mm} / \mathrm{s}$ when machining on glass and $0.25 \mathrm{~mm} / \mathrm{s}$ when machining on PMMA using a programmable computer-controlled linear stage with a positioning resolution of $0.5 \mu \mathrm{m}$. The powder mass flow rate was $1.6 \mathrm{~g} / \mathrm{min}$ and $2.4 \mathrm{~g} / \mathrm{min}$ for glass and PMMA, repectively .

A range of deeper channels were machined with the nozzle tilted at $\theta=30^{\circ}$ in a plane perpendicular to the direction of machining (Fig. 3.1b) using a standoff distance of $10 \mathrm{~mm}$ on both glass and PMMA samples. The channel cross-sections were measured using an optical profilometer (NANOVEA ST400 Micro Photonics Inc., Irvine, CA, USA) having a depth resolution of $25 \mathrm{~nm}$ and a lateral resolution of $0.1 \mu \mathrm{m}$. All experiments were conducted twice; i.e. at each standoff distance, two separate channels were machined without turning of the microblaster to ensure that the mass flow rate remained the same, and three cross-sections were measured on each channel. Channels of different depth were machined using a stepped arrangement, whereby the channel was divided into 5 parts corresponding to $1,3,5,7$, and 9 nozzle passes.

\subsubsection{Channels in rods}

Channels were machined axially in two sizes of glass rod (borosilicate, Swift Glass Co. Inc., Elmira, NY, USA) and one size of PMMA rod (type CLR, EXTRUDED ACRYLIC ROD, Piedmont Plastics, Scarborough, ON, Canada) at a $10 \mathrm{~mm}$ nominal nozzle standoff (Fig. 3.1c). The powder mass flow rate was kept at $1.8 \mathrm{~g} / \mathrm{min}$. All other process conditions were as given in Section 3.3.2. The average diameter ( \pm standard deviation of 8 measurements) over an $11 \mathrm{~cm}$ length was $4.99 \pm 0.04 \mathrm{~mm}$ and $2.98 \pm 0.03$ for glass, and $4.72 \pm 0.01 \mathrm{~mm}$, for PMMA. To ensure that the rod was aligned with the center of the abrasive jet and was parallel to the stage traverse during machining, the rod was taped into a groove that had been previously machined on a flat glass plate clamped to the stage (Fig. 3.1c). Prior to scanning the cross-sections of the machined channels with the optical profilometer, the surface of the rod was coated with a thin layer of soot using a candle to reduce the lateral reflection of light at the periphery of the rods and increase the accuracy of the measurements. To ensure that the rod was also aligned with the profilometer scan direction, cross-sectional scans were made across 4 different un-eroded sections, and the rod was 
re-aligned manually until 4 such profiles were coincident to within $0.01 \%$. A bracket was then attached to the profilometer stage to preserve the alignment for subsequent measurements.

\subsection{Results and discussion}

\subsubsection{Erosive efficacy function}

The average value of $\beta$ in Eq. (3.16) was 28, which was obtained from the best fits (based on the least-squares method) to the dimensionless (i.e. depth per unit maximum depth) profiles of the shallow channels shown in Fig. 3.6, that were machined on a flat surface at perpendicular incidence with nozzle standoffs $h=10 \mathrm{~mm}, 15 \mathrm{~mm}$ and $20 \mathrm{~mm}$. The fits at each standoff were good, with regression coefficients $\left(R^{2}\right)$ greater than 0.99 . In order to account for mass flow rate fluctuations, the value of $\delta$ was scaled to match the first pass profile depth in the surface evolution equation (Eqs. (3.13) and (3.15)). The value of $\beta$ characterized the erosive efficacy function that was used to model the channels machined on curved surfaces and at oblique incidence on flat targets. As mentioned in Section 3.3.2, the experiments were repeated twice at each standoff distance and the overall standard deviation of all 6 measurements of $\beta$ was 1.5 (5.4\%). This small deviation verified that particles traveled in approximately straight lines from the nozzle to a flat surface, confirming the assumption of the conical shape of the abrasive air-jet plume (Fig. 3.4), and lent confidence to the assumption that the effect of particle velocity variations across the jet on the erosive efficacy was negligible. In the absence of unexpected aerodynamic or particle rebound effects, the erosive efficacy on arbitrarily shaped and oriented targets could be determined as the intersection of the target surface and this three-dimensional function. 


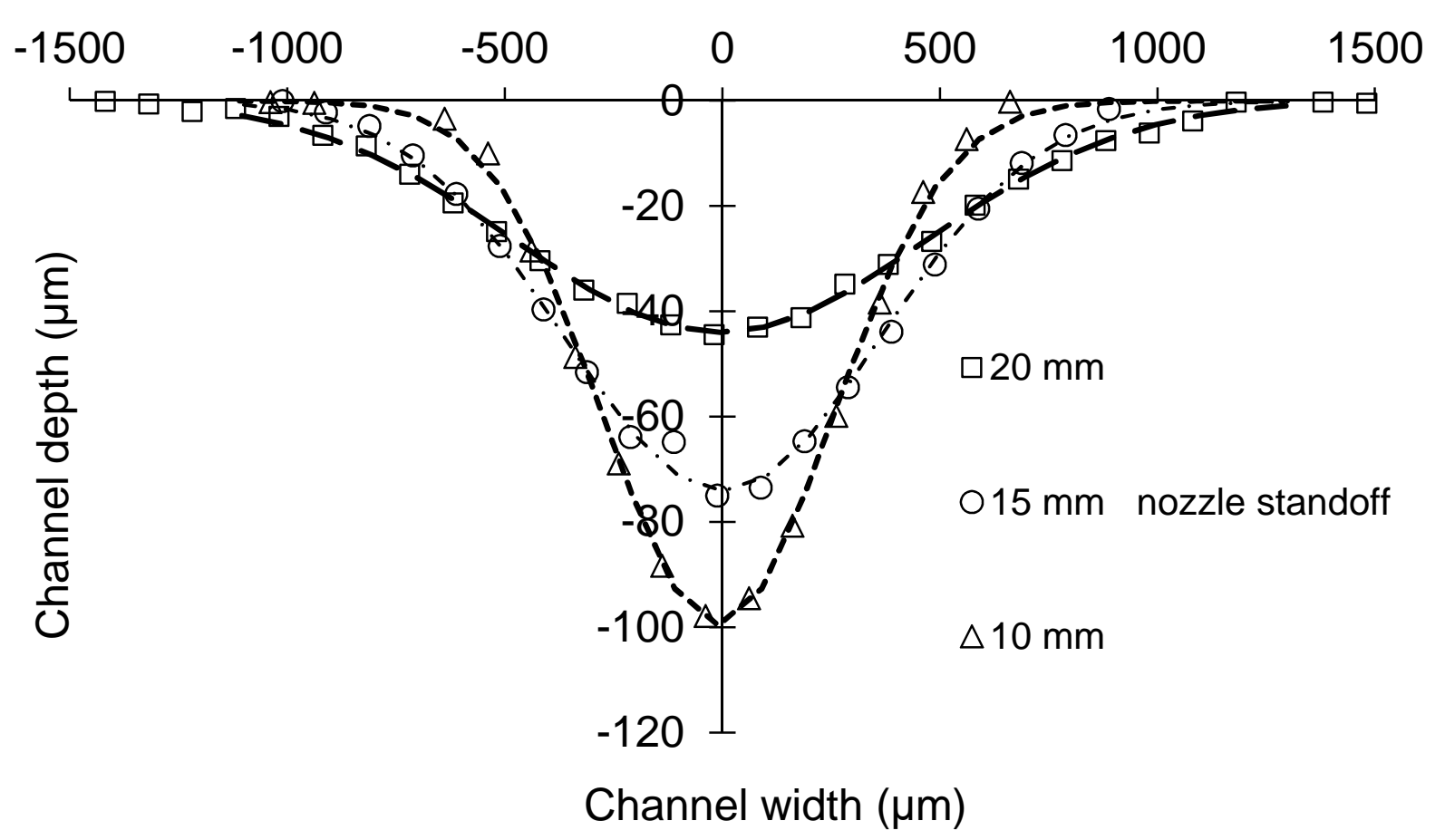

Fig. 3.6 Measured profiles (symbols) and curve fits (dashed lines) of three shallow channels machined at $10 \mathrm{~mm}, 15 \mathrm{~mm}$ and $20 \mathrm{~mm}$ nozzle standoffs on a flat glass surface.

\subsubsection{Prediction of channel profiles using new model}

\subsubsection{Channels in tilted flat glass target}

The new model of Eq. (3.13) for surface evolution in brittle materials was used with the three-dimensional erosive efficacy function, Eq. (3.16) with $\delta$ obtained by matching Eq. (3.13) to measured profile for the first pass and $\beta=28$ determined from the perpendicular incidence flat surface profiles (Section 3.4.1), to predict the profiles of channels machined on tilted flat glass surfaces. Equation (13) was solved numerically using the method of lines in Mathcad 15 (PTC Inc. Needham, MA, USA), using the erosive efficacy obtained from Eq. (3.16) with Eq. (3.18) as the initial condition. The number of spatial $(\mu \mathrm{m})$ and time (s) discretization points used was 200 and 300 respectively, which ensured the accuracy and convergence of the numerical solution. 


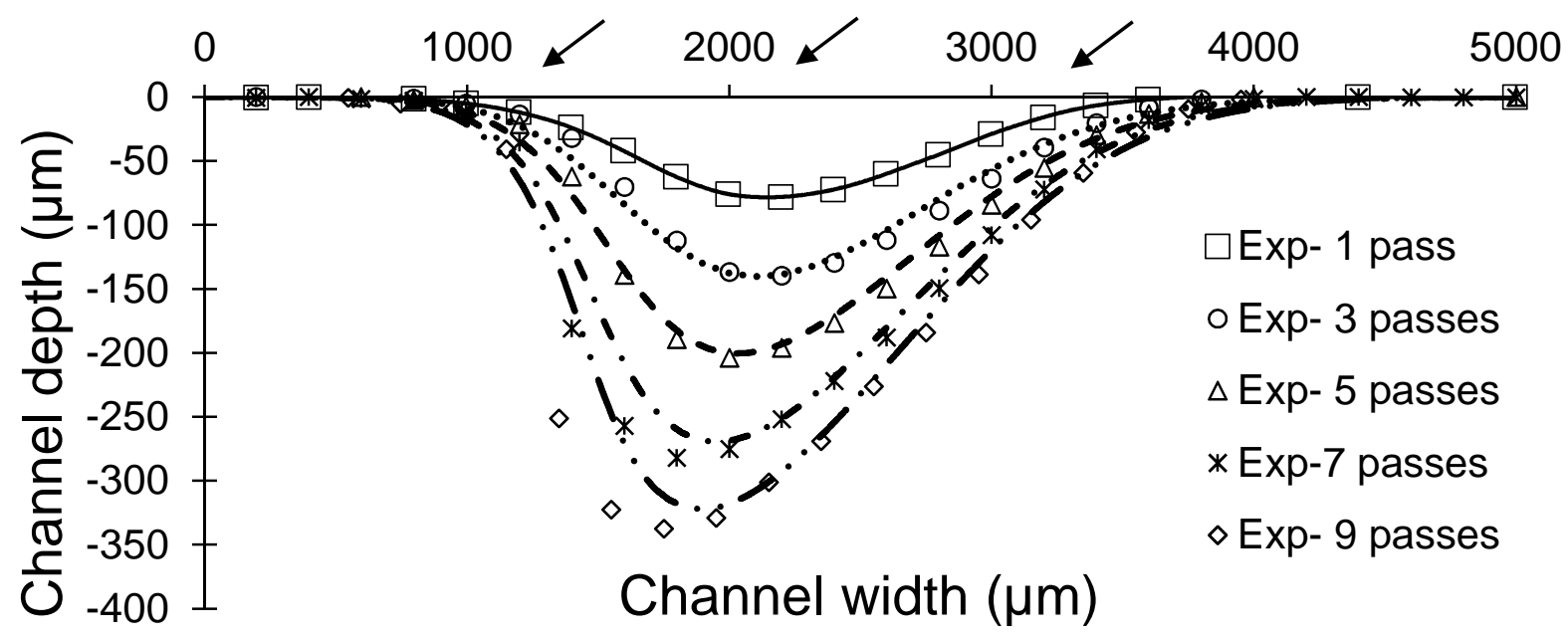

Fig. 3.7 Measured (symbols) and predicted (dashed lines) profiles of channels machined on glass at a nozzle angle of $30^{\circ}$. Predictions used the new model, Eq. (3.13), with the three-dimensional erosive efficacy, Eq. (3.16) (Section 3.2.3). The maximum standard deviation of 6 measurements (as explained in Section 3.3.2) at the bottom of the channels was $16.5 \mu \mathrm{m}$, for the $9^{\text {th }}$ pass. Arrows show direction of nozzle inclination. Note difference in depth and width scales.

The predicted channel profiles were translated and rotated to align the uneroded surfaces. Fig. 3.7 shows there was good agreement between the measured and predicted profiles of asymmetric channels machined in flat glass at a nozzle angle of $30^{\circ}$. For example, the predicted centerline depth after 5 passes was $3 \%$ less than the measured result, increasing to $7.5 \%$ for the $9^{\text {th }}$ pass. As will be discussed in Section 3.4.2.2, the slight under-prediction of erosion on the left sidewall was most probably due to fluctuations in abrasive mass flow rate for the relatively rapidly eroding glass. 


\subsubsection{Channels in tilted flat PMMA target}

Fig. 3.8 shows that the measured profiles of channels machined on a flat surface of PMMA at an angle of $30^{\circ}$ were closely matched by the profiles predicted by the new model of Eq. (3.15) with the three-dimensional erosive efficacy, Eq. (3.16), with $\delta$ and $\beta$ obtained as described in the previous section. For example, the predicted centerline depth after 5 passes was $0.08 \%$ lower than the measured value, reaching 3\% for the 9th pass. The better fit between the predicted and measured profiles for PMMA, than glass (Fig. 3.7), could be attributed to the lower etch rate (i.e. centerline depth increment per dose of particles impinged on the target) in PMMA $(131 \mu \mathrm{m} / \mathrm{g})$ in comparison to glass $(286 \mu \mathrm{m} / \mathrm{g})$, which made it less susceptible to fluctuations in abrasive mass flow rate.

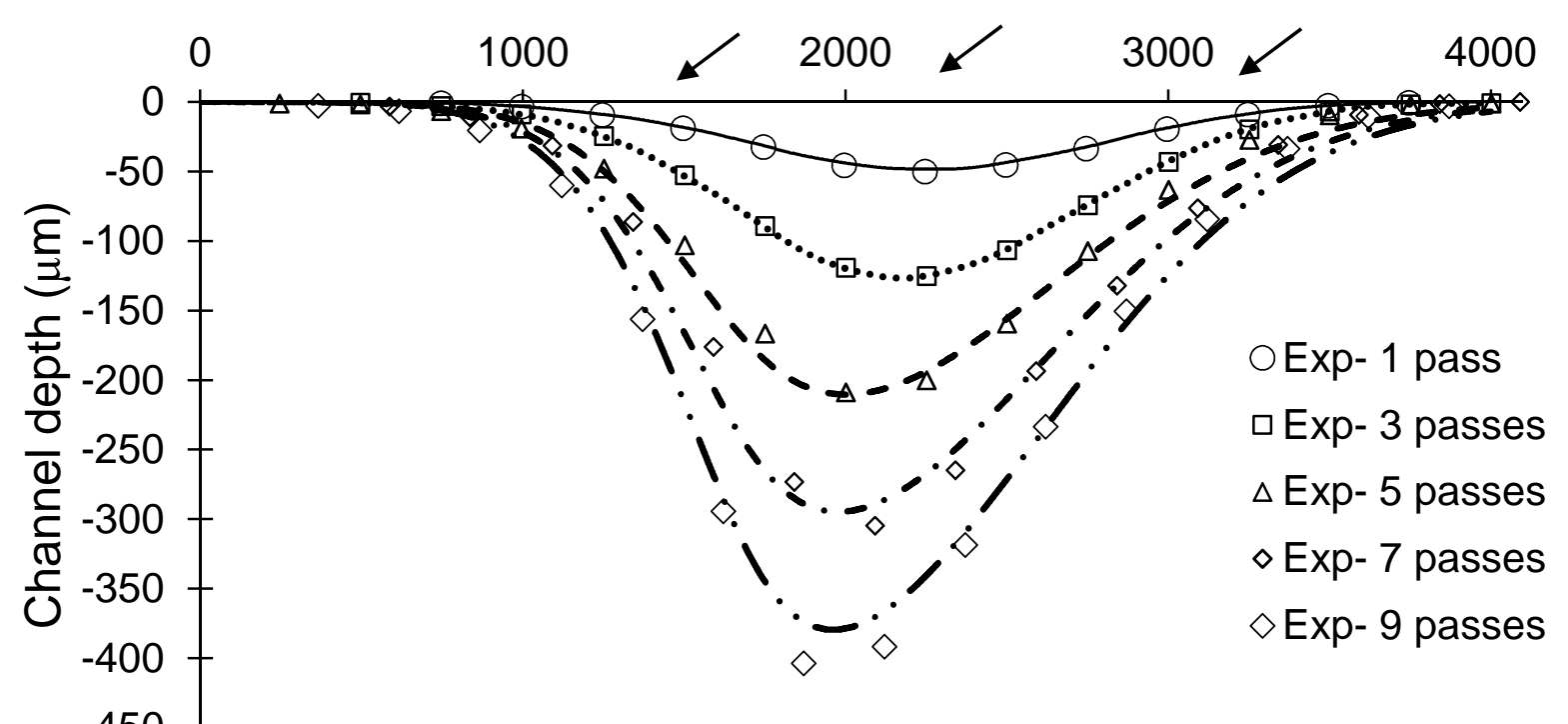

Channel width $(\mu \mathrm{m})$

Fig. 3.8 Measured (symbols) and predicted (dashed lines) profiles of channels machined in PMMA at a nozzle angle of $30^{\circ}$. Predictions used the new model, Eq. (3.15), with the threedimensional erosive efficacy, Eq. (3.16) (Section 2.3). The maximum standard deviation of 6 measurements (as explained in Section 3.3.2) at the bottom of the channels was $14 \mu \mathrm{m}$, for the

9 th pass. Arrows show direction of nozzle inclination. Note difference in width and depth scales. 


\subsubsection{Channels in glass rods}

The model of Eq. (3.15) and the erosive efficacy function of Eq. (3.16) with $\delta$ (obtained by matching Eq. (13) to measured profile of the first pass) and $\beta=28$ (flat surface, perpendicular incidence) were initially used to predict the cross-sectional profiles of channels machined axially in the $3 \mathrm{~mm}$ and $5 \mathrm{~mm}$ diameter glass rods. As with the tilted flat surfaces, the inherent assumption was that the footprint would simply be the intersection of the three-dimensional erosive efficacy function with the rod geometry. However, it was found that such an approach yielded significant under-predictions of the channel width. In this case, the value of the jet focus coefficient, $\beta$, for the erosive efficacy was obtained by adjusting it in the surface evolution model to fit the measured first path profile. For the $5 \mathrm{~mm}$ and $3 \mathrm{~mm}$ rods, the values of $\beta$ calculated in this manner were 22 and 20, respectively, instead of 28 as found in Section 3.4.1 for flat surfaces; i.e. compared to the flat target case, $\beta$ decreased by $21 \%$ for the $5 \mathrm{~mm}$ rod and $28 \%$ for the $3 \mathrm{~mm}$ rod. These values of $\beta$ were used in the model for the prediction of channel profile evolution in the corresponding diameter rod.

The decrease in $\beta$ with decreasing rod diameter indicated that the jet was behaving as if it diverged more when it interacted with smaller diameter rods, such that the degree of additional spreading of the particles compared with the flat target increased as the rod became smaller. It was hypothesized that this was due to rebounding particles that struck the rod a second time beyond the primary footprint of the jet. This hypothesis was examined by constructing a simplified model of the flow field and particle trajectories using computational fluid dynamics (CFD) simulations (ANSYS Fluent 14.0, ANSYS Inc., Cecil Township, PA, USA). Three-dimensional, single-phase, steady domains were meshed using quadrilateral $5 \mu \mathrm{m}$ elements. The realizable $\kappa-\varepsilon$ turbulence model was used, following the AJM modeling work of Li et al. [55], and the models converged with residuals below 10-3. The particles were given a shape factor of 0.76 as measured by Dehnadfar et al. [56], and were released at various points across the nozzle exit plane from rest and then tracked using the Lagrangian discrete phase model. The particle velocities and average flux were adjusted to match those used in the experiments (Section 3.3). However the model did not attempt to capture the variation in the local flux, velocity and divergence (particle trajectory) across the jet that has been measured using particle capture [54] and particle image velocimetry [56]. These variations are caused by the interactions between the particles and the walls of the 
nozzle as described by [57]. This simplification of the model was justified by our interest in the fate of rebounding particles. The normal and tangential restitution coefficients for particle to target collisions were set to 0.2 in the CFD simulations as suggested by Slikkerveer and in't Veld [22] for similarly sized $\mathrm{A} 12 \mathrm{O} 3$ particles impacting glass.

Figure 3.9 compares the particle trajectories in the jet footprints on a flat glass target, and the $5 \mathrm{~mm}$ and $3 \mathrm{~mm}$ glass rods. It is seen that as the curvature increased the footprint also increased from approximately $2.05 \mathrm{~mm}$ wide for a flat surface, to approximately $2.35 \mathrm{~mm}$ wide for the $3 \mathrm{~mm}$ rod, due to the rebounding particles that were driven back toward the surface to strike again. The CFD modelling also established that these second-strike particles had sufficient kinetic energy to erode the glass. For example, on the flat surface, the second strikes were at a velocity of approximately $30 \mathrm{~m} / \mathrm{s}$ as measured from CFD. Although the kinetic energies of such impacts were below the $39 \mathrm{~nJ}$ ductile-brittle threshold in glass for $10 \mu \mathrm{m}$ alumina abrasives given by Slikkerveer et al. [46], they were sufficient to cause plastic deformation and ductile erosive wear, as reported by Nouraei et al. [58] using the same particles at similar energies. Therefore, the second strike of rebounding particles is a plausible explanation for the increase in the measured focus coefficient $\beta$.

As explained above, the CFD model launched particles uniformly across the exit plane of the nozzle without regard to the effects of the interactions between the particles and the walls of the nozzle that spread the particles in the jet and create variations in the flux and velocity across the jet. Therefore, the apparent footprint of the primary jet in Fig. 3.9 is smaller than it actually is, and in reality the zone of primary impacts overlaps at least a portion of the peripheral region containing most of the second-strikes. Therefore, the measured $\beta$ inferred from the eroded profiles on a flat surface in Fig. 6 reflects both the first and second strikes. As shown by the analysis of Section 3.2.2 and Fig. 3.9b, the curvature of the target intensified the variation in the local impact angle of both first and second strikes. To account for this, the effective $\beta$ used in the surface evolution equations for curved surfaces had to be decreased (wider jet). According to the above discussion and Section 3.2.3, Eq. (3.16) with the effective nozzle focus coefficient, $\beta$, is representative of the combined effects of the second strikes from rebounding particles and the actual mass flux, particle velocity distribution, and divergence of particle trajectories across the incident jet that give rise to the primary first-strike footprint. 
In summary, the jet footprints became wider due to particle rebound and second-strike, and this widening was captured in the model by the value of the jet focus coefficient, $\beta$, in the erosive efficacy (Eq. (3.16)).
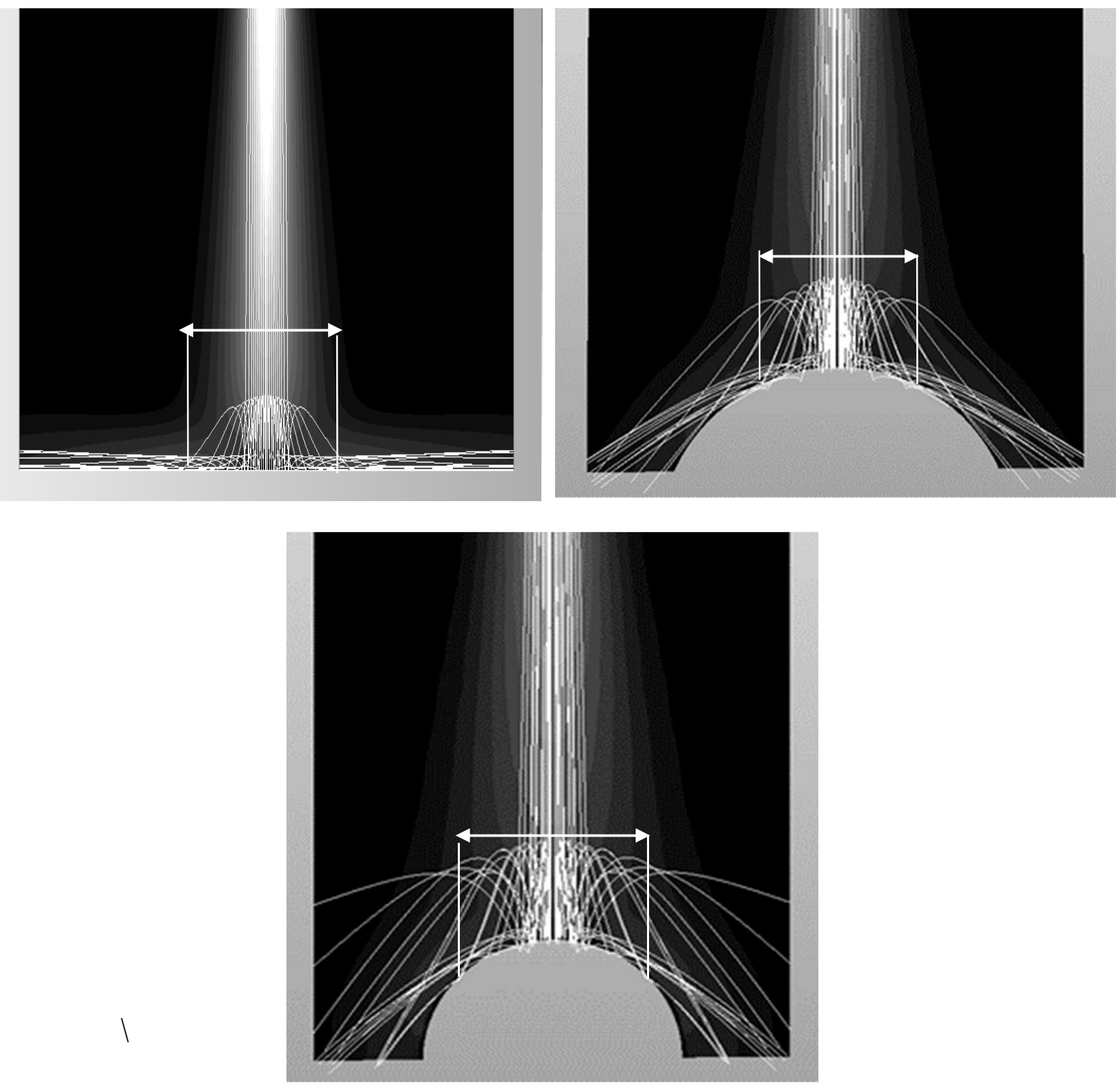

Fig. 3.9 CFD results illustrating the effect of particle second strike on (a) flat target (b) $5 \mathrm{~mm}$ and (c) 3 $\mathrm{mm}$ glass rods. The distance from inlet to target was $10 \mathrm{~mm}$ in all cases. 
Fig. 3.10 shows that there was quite good agreement between the predicted and measured profiles, with errors in the predicted center depth of $6.4 \%$ for the $3^{\text {rd }}$ pass and $8.2 \%$ for the $7^{\text {th }}$ pass on the $3 \mathrm{~mm}$ diameter rod. For the $5 \mathrm{~mm}$ diameter glass rod, the model over-predicted the center depth by $3.5 \%$ for the $9^{\text {th }}$ pass, while it under-predicted the center depth by $5.6 \%$ for the $11^{\text {th }}$ pass. The origin of the depth $Z$ in these graphs was chosen as the boundary between the eroded and uneroded portions of the cylinder.

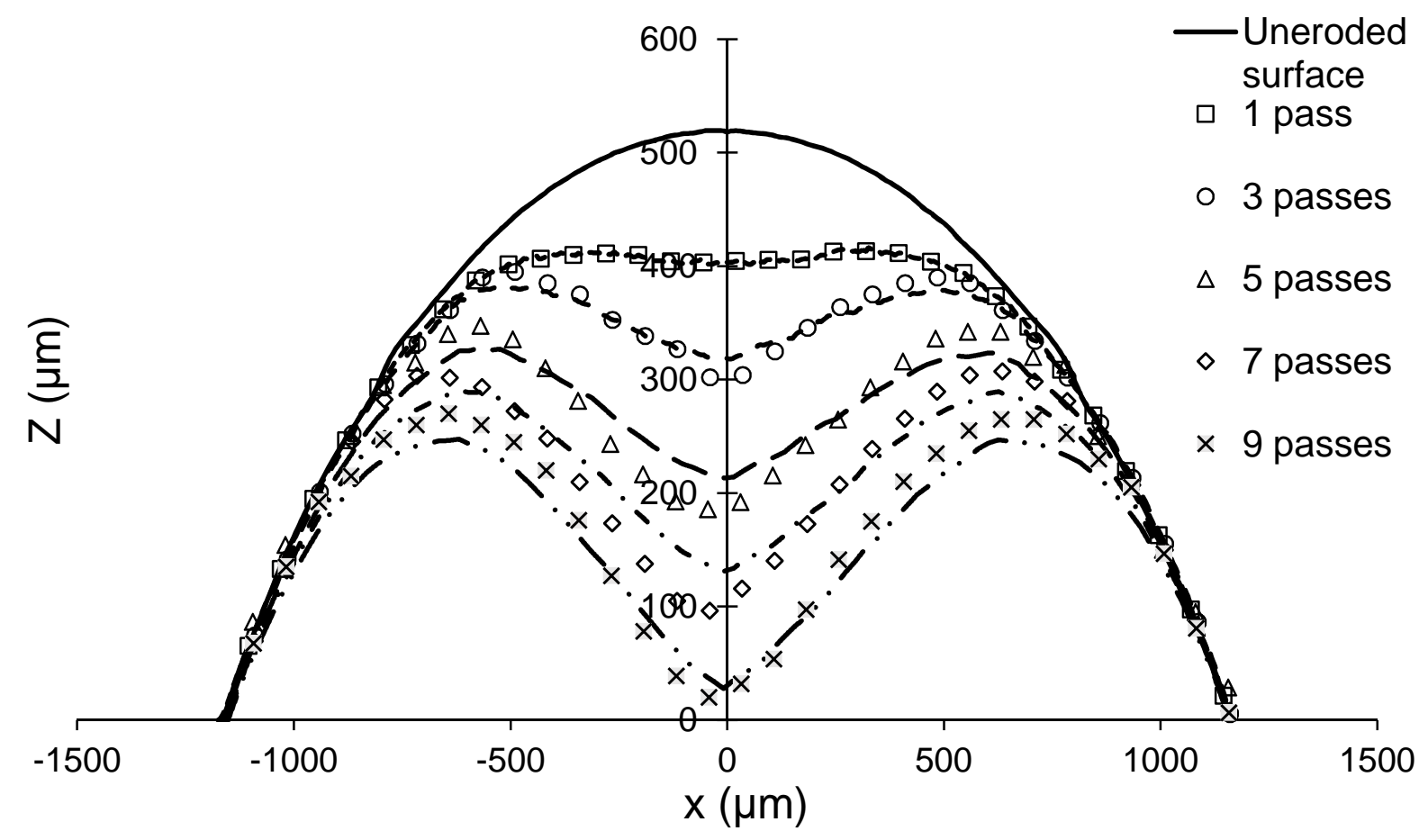

(a) 


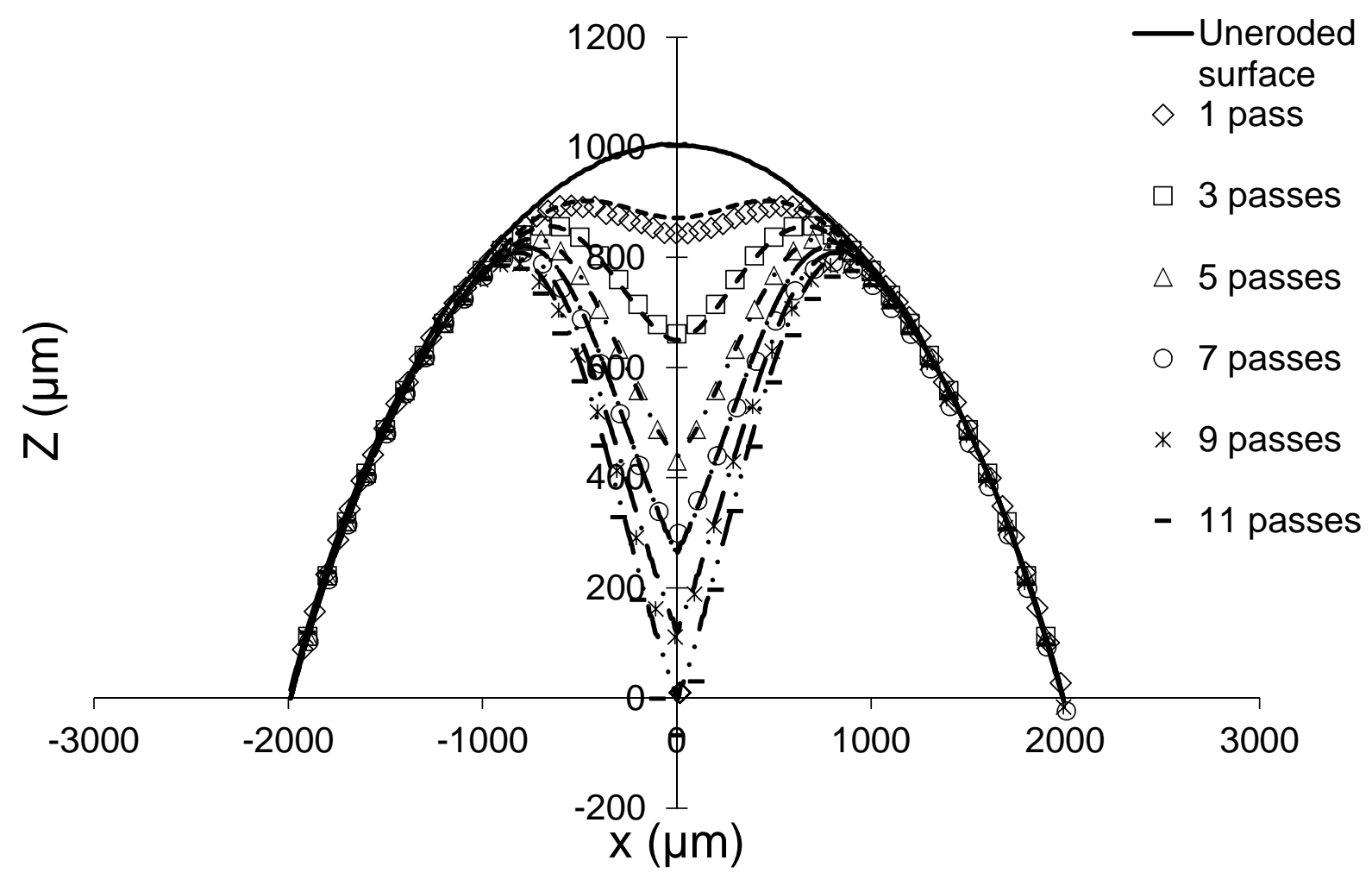

(b)

Fig. 3.10 Measured cross-sectional profiles (symbols) of channels machined axially along glass rods of diameter: (a) $3 \mathrm{~mm}$, and (b) $5 \mathrm{~mm}$ compared with predictions (solid lines) of Eq. (3.13) using the three-dimensional erosive efficacy where $\beta=20$ and 22 for $3 \mathrm{~mm}$ and $5 \mathrm{~mm}$ rod, respectively, Eq. (3.16). The maximum standard deviation of 6 measurements (explained in Section 3.3.2) at the channel center line depth was $20 \mu \mathrm{m}$ for the $7^{\text {th }}$ pass and $33 \mu \mathrm{m}$ for the $5^{\text {th }}$ pass for $3 \mathrm{~mm}$ and $5 \mathrm{~mm}$ rod, respectively. 


\subsubsection{Channels in PMMA rod}

Fig. 3.11 shows that the measured channel cross-sections made in $4.72 \mathrm{~mm}$ diameter PMMA rods agreed very well with those predicted using the proposed surface evolution model, Eq. (3.15), with a maximum error of $1.2 \%$ in the $11^{\text {th }}$ pass. As with the $5 \mathrm{~mm}$ diameter glass rods, Eq. (3.16) was used as the erosive efficacy with $\beta=22$. The small discrepancies between the predicted and measured profiles at the peaks and centerline could again be attributed to fluctuations in the abrasive mass flow rate. As explained in Section 3.4.2.2, the slightly better agreement between the predictions and the measured profiles in PMMA was due to its smaller erosion rate compared to glass which made it less susceptible to these variations.

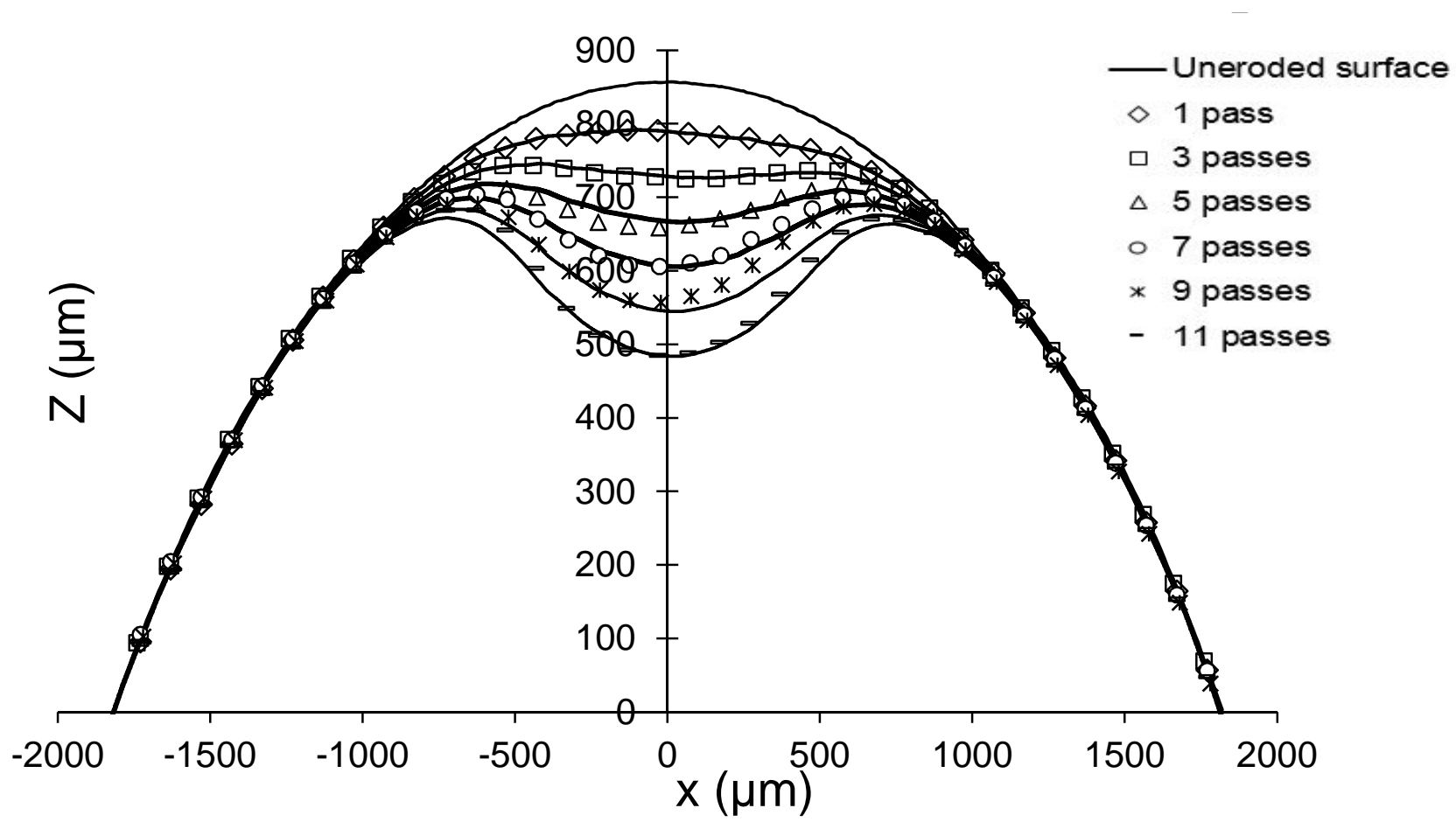

Fig. 3.11 Measured cross-sectional profiles (symbols) of channels machined axially in $4.72 \mathrm{~mm}$ diameter PMMA rod compared with predictions (solid lines) of Eq. (3.15) using the threedimensional erosive efficacy where $\beta=22$, Eq. (3.16). The maximum standard deviation of 6 measurements (explained in Section 3.3.2) at the channel center line depth was $6.25 \mu \mathrm{m}$ for the $7^{\text {th }}$ pass. 


\subsubsection{Predicted channel profiles using previous models}

The measured cross-sectional profiles of asymmetric channels machined in a flat glass plate at $30^{\circ}$ (Fig. 3.1b) are shown in Fig. 3.12, along with the corresponding predictions of the previous surface evolution model of ref. [30] which neglected jet divergence and local standoff variations, and used the mapping approach to determine the erosive efficacy function; i.e. Eq. (3.2) was the surface evolution equation. Also shown are the predictions of the present model as implemented using the procedures described in the following section. It is seen that the present model was more accurate than that of [30], particularly in capturing the erosion occurring on the near-side of the channel. The relative magnitudes of the effects of jet divergence and the variation of local standoff distance were assessed by running the present model in two ways. The effect of jet divergence was examined by replacing $z$ with $h_{0}$ (nominal standoff distance) in Eqs. (3.11) and (3.16). The effect of standoff variation was measured by assuming $\gamma$ to be $0^{\circ}$ in Eq. (3.10). It was observed that the neglect of each effect increased the skewness of the predicted channel profiles (i.e., under-predicted near wall and over-predicted far wall). Moreover, ignoring the change in local standoff produced a 12\% over-prediction of the channel depth in glass and $8 \%$ in PMMA after nine nozzle passes, with the error increasing further with depth. In comparison, the neglect of the spreads in the jet resulted in a $2 \%$ over-prediction of channel depth in glass and $1.5 \%$ in PMMA after nine nozzle passes. The greater influence of the local standoff correction is consistent with the observation in Section 3.2.2 that the there was a 35\% variation of nozzle standoff across a channel machined with $h_{0}=10 \mathrm{~mm}$ and $\theta=60^{\circ}$, compared with an $18 \%$ variation of local impact angle across the channel width.

Fig. 3.13 compares the predictions of ref. [9] and the present model with the measured cross-sections of channels machined in a $5 \mathrm{~mm}$ glass rod at $90^{\circ}$. In this case, the erosive efficacy function used in both models was determined using the best fit to the profile of a single shallow channel machined at $90^{\circ}$ in the rod [9]. In contrast, the model of [30] obtained the erosive efficacy by applying a coordinate transformation which mapped each point of a shallow channel profile machined at $90^{\circ}$ to a point on a tilted surface. Fig. 3.13 shows that the model of [9] failed to predict the channel profiles machined in the glass rod. This was due to two factors: First, the assumed erosive efficacy determined from the first-pass profile violated the requirement of a zero slope (Section 3.2.3) at the edges of the footprint, because of the rod curvature. Secondly, the 
model of [9] neglected changes in local standoff and impact angle due to the spreading of the jet. The assumption of constant standoff distance on the $5 \mathrm{~mm}$ glass rod after 5 nozzle passes led to an $8.5 \%$ error in the predicted channel depth, and the neglect of jet spread caused an error in the depth of $2 \%$.

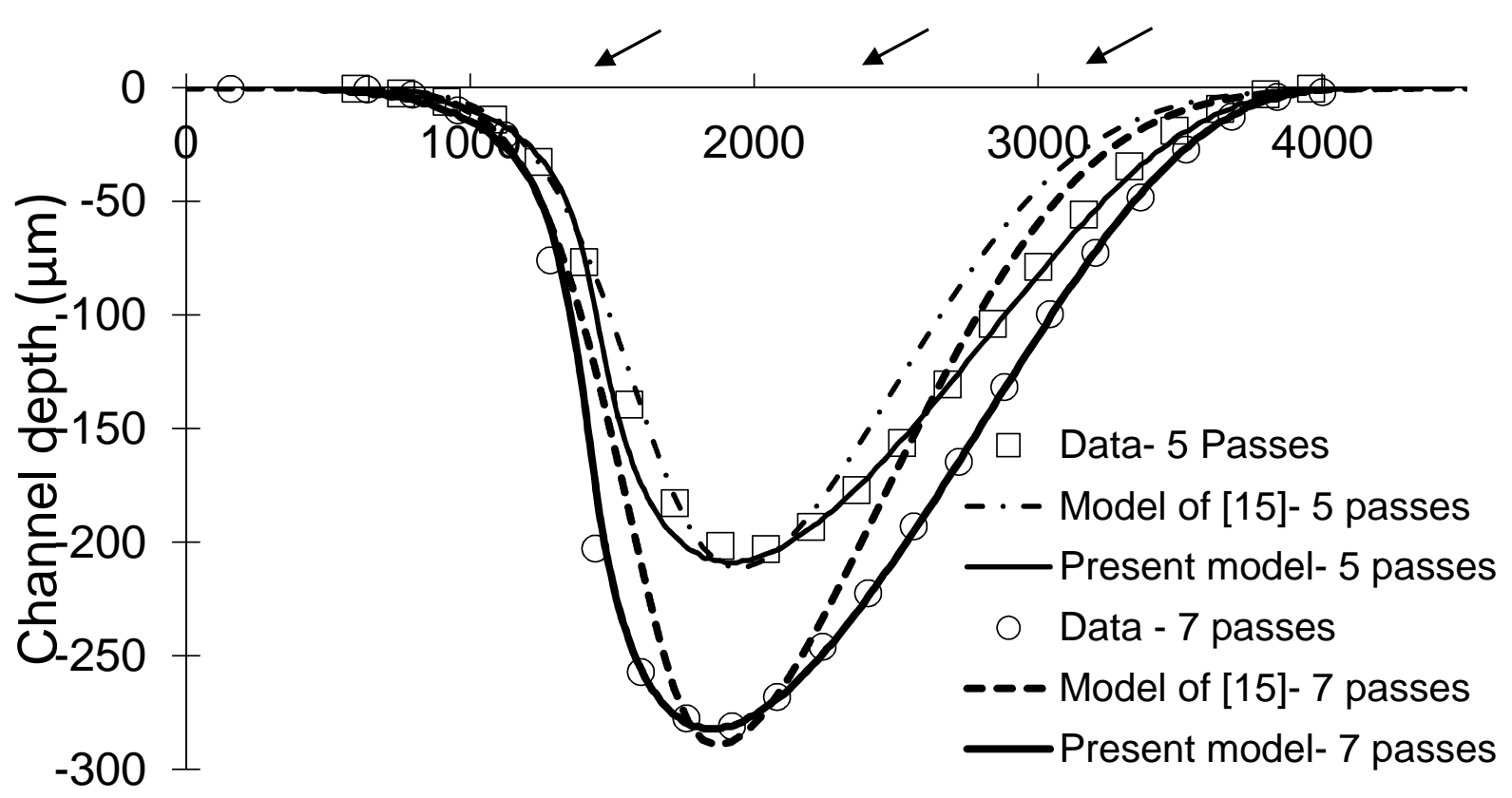

Channel width $(\mu \mathrm{m})$

Fig. 3.12 Comparison of measured asymmetric channel cross-sections in a flat glass plate with predictions of present model (Eq. (3.13)) and that of ref. [30]. Arrows show direction of incident particles at a jet angle of $30^{\circ}$. 


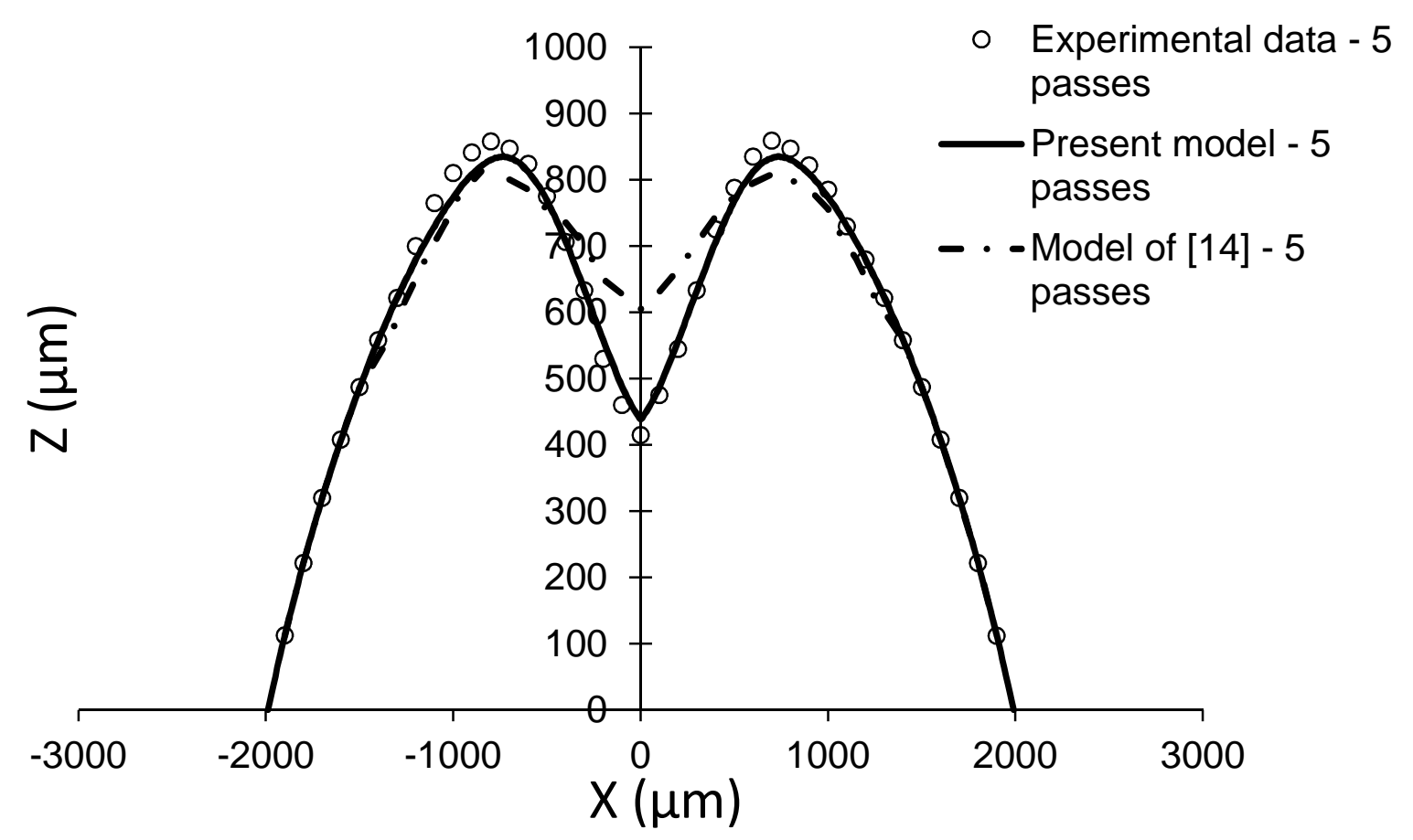

Fig. 3.13 Comparison of measured channel cross-sections in a $5 \mathrm{~mm}$ diameter glass rod with predictions of present model (Eq. (3.13)) and model of ref. [9].

\subsection{Summary}

An existing surface evolution model was modified by taking into account the change in local nozzle standoff distance and local impact angle of particles striking flat, tilted surfaces and highly-curved rods. It was observed that model predictions and experimental data matched quite well with a maximum error about $8 \%$. An investigation of the effect of each modification independently revealed that neglect of local standoff distance contributed to greater error in predicting the channel profiles than did ignoring the spread of particles across the channel width.

The measurement of erosive efficacy on a flat surface at perpendicular incidence using three nozzle standoff distances confirmed that the jet divergence could be modeled approximately as a cone. For tilted flat surfaces, the intersection of this cone with the surface was sufficient to 
accurately characterize the erosive efficacy. However, this was not the case for the highly-curved surfaces, since the nozzle focus coefficient for curved surfaces was smaller than for flat targets, indicating that the nozzle was behaving as if it diverged more on curved surfaces. Computational fluid dynamics modeling showed that this was caused by differences in the second strike of rebounding particles that tended to widen the jet footprint on a curved surface more than on a flat.

As a general rule, a surface should be considered highly curved if the difference between the local standoff at the edge of the jet footprint and at the centerline is at least $5 \%$ of the standoff at the jet axis (centerline). As an illustration, for the present jet and $5 \mathrm{~mm}$ glass rod, assuming a constant standoff distance resulted in a $6 \%$ over-prediction of erosion at the edge of the footprint. The presented model in principal can be used to model profile development on a wide variety of surface geometries with a simple change in initial conditions. However, caution must be exercised since the apparent divergence of the jet may depend on the target geometry, due to possible differences in particle second strike trajectories.

The findings of this chapter served as motivation to investigate the effect of particle second strikes on the foot print size more closely in Chapter 4. As will be shown, computational fluid dynamics and further experimentation can be used as an alternative technique to obtain the nozzle focus coefficient, $\beta$, in the erosive efficacy expression. 


\section{Chapter 4 Prediction of the erosive footprint in the abrasive jet micro- machining of flat and curved glass}

This chapter is based on the following published paper:

K. Kowsari, *A. Nouhi, V. Hadavi, J.K. Spelt, M. Papini, "Prediction of the erosive footprint in the abrasive jet micro-machining of flat and curved glass", Tribology international, vol. 106, pp. 101-108, 2017.

* Nouhi conducted the AJM and shadowgraphy experiments and carried out the experimental measurements and mathematical calculations. Kowsari led the development of the CFD simulations, and led the writing of the paper. Hadavi helped analyze the shadowgraphy results.

\subsection{Introduction}

As demonstrated by, for example, Solignac et al. [59] and Liu et al. [60], abrasive jet micro-machining (AJM) can be used to machine micro-features in brittle and ductile materials. In AJM, a small, high-speed air jet is used to accelerate fine abrasive particles which are made to impact and erode the target material. The topography of the eroded surface depends strongly on the distribution of the velocity and number density of the impacting particles, termed the erosive efficacy within the footprint of the jet on the surface, as defined by Ghobeity et al. [9]. AJM erosive footprints have thus far been inferred from the profile of shallow eroded scars on flat targets. For example, Ghobeity et al. [9](2008) found that at a typical standoff distance of $20 \mathrm{~mm}$ between the nozzle exit plane and target, the footprint diameter was approximately 3 times wider than the jet diameter, and resulted in a roughly $\mathrm{V}$-shaped eroded topography in glass, indicative of a maximum erosive efficacy near the jet centerline. Using an analytical model, Ghobeity et al. [29] demonstrated that the shape and depth of machined micro-channel profiles made through an erosion-resistant mask were affected by the abrasive particle size distribution and the width of the mask opening. Dehnadfar et al. [56] implemented a pulsed laser shadowgraphy method to measure the abrasive particle size and velocity distribution in both a free jet and through a mask opening. The shadowgraphy measurements were in good agreement with the analytical model of Ghobeity et al. [29].

Shipway [61] measured the depth profiles of wear scars to investigate the effect of particle divergence in an abrasive jet plume for relatively large nozzles ( $4.93 \mathrm{~mm}$ diameter). He found that the distribution of particle trajectories followed a gamma distribution, and observed that the local 
impact angle due to the plume divergence needed to be taken into account to obtain an accurate prediction of the erosion on a flat surface. Using a particle capturing technique, Burzynski and Papini [54] found that the spatial distribution of abrasive particles within a micro-abrasive jet produced by nozzles having diameters between $460 \mu \mathrm{m}$ and $1.5 \mu \mathrm{m}$ followed a Weibull distribution. More recently, Mansouri et al. [62](2015) modeled abrasive jet flows using computational fluid dynamics (CFD) and showed secondary impacts of the particles after rebounding from a flat target, However, that study focused on sand blasting, which involved much larger length scales ( $7 \mathrm{~mm}$ nozzle diameter and particle sizes of 150-300 $\mu \mathrm{m}$ ) than those used in AJM (460-760 $\mu \mathrm{m}$ nozzle diameters of particle sizes of 10-25 $\mu \mathrm{m})$. None of these earlier studies quantified or discussed the effect of particle second strikes on the footprint size.

More recently, Qi et al. [63] used CFD to model the flow field and particle trajectories in ultrasonic vibration-assisted abrasive slurry jet micro-machining of glass and found an increase in the erosion rate due to target vibration. Moreover, Qi et al. [64] and Kowsari et al. [65] obtained the erosive footprints from CFD for use in surface profile models. However, the trajectories and erosive patterns caused by the second strikes in these water slurries were significantly different from those in air-driven jets due to the large difference in the viscosities of water and air. The only AJM study involving CFD models of particle secondary strikes was by Nouhi et al. [66]. While studying the effect of the variation in the local nozzle standoff distance and divergence angle of particle trajectories in the jet plume on the erosion of cylinders, they found that the apparent erosive footprint size changed depending on the surface curvature. The results of their preliminary CFD study revealed that this change in footprint size was due to differences in particle second strike locations brought about by the target curvature. That observation provided the motivation for the present work, which considers the effect of second strike on footprint size in detail.

In summary, the effect of secondary particle impacts in AJM remains largely unexplored, and the present work aims to characterize and numerically predict the AJM footprint at various standoffs using CFD models.

\subsection{Experiments}

An AccuFlo AF10 Micro-Abrasive Blaster (Comco Inc., Burbank, CA, USA), described in detail in Dehnadfar et al. [56](2012) was used in all the experiments. The air pressure upstream of the $760 \mu \mathrm{m}$ inner-diameter nozzle (length-to-diameter ratio of $\sim 6.6$ ) was $200 \mathrm{kPa}$, and 
aluminum oxide $\left(\mathrm{Al}_{2} \mathrm{O}_{3}\right)$ powder (Comco Inc., Burbank, CA, USA) having a nominal diameter of $10 \mu \mathrm{m}$ (log-normal distribution with a standard deviation of $3.31 \mu \mathrm{m}$ ) was used in all the experiments. The powder mass flow rate was $2.7 \mathrm{~g} / \mathrm{min}$.

\subsubsection{Jet and footprint measurements}

As will be shown in Section 4.3.2, the jet footprint could be viewed as the superposition of a primary particle plume originating from the nozzle, and a secondary plume consisting of particles that rebounded from the surface and struck a second time. The jet divergence was measured using a digital camera attached to a microscope having a field of view of $3 \times 2 \mathrm{~mm}$. The diameters of the net footprints (including both primary and secondary particle impacts) were inferred by using an optical profilometer (NANOVEA ST400 Micro Photonics Inc., Irvine, CA, USA, depth resolution of $25 \mathrm{~nm}$; lateral resolution of $0.1 \mu \mathrm{m}$ ), to measure the shallow eroded profile resulting from jet impingement on flat $100 \times 50 \times 3 \mathrm{~mm}$ thick glass (Borofloat, Swift Glass Co. Inc., Elmira, NY, USA) targets. Experiments were performed at standoffs (distance between nozzle exit and target) of 5, 10, 20, and $30 \mathrm{~mm}$ at a perpendicular, stationary jet incidence. A shallow eroded profile on a 5 mm diameter glass rod (Borofloat, Swift Glass Co. Inc., Elmira, NY, USA) at a $10 \mathrm{~mm}$ standoff distance under similar blasting conditions was measured previously in Nouhi et al. [66].

To determine the footprint diameter resulting from only the primary plume originating at the nozzle, the jet was made to impact $100 \times 100 \times 0.1 \mathrm{~mm}$ thick sheets of multi-purpose paper (Canon Canada Inc., Mississauga, ON, Canada) such that the impacting particles pierced the paper, but did not rebound. A small amount of tension was applied to the paper to prevent it bending due to the particle impacts. The size of the primary plume was measured using a microscope with a field of view of $6 \times 4 \mathrm{~mm}$.

The divergence of the abrasive particles in the air jet was studied further using doublepulsed laser shadowgraphy as explained in detail in Dehnadfar et al. [56] and Hadavi et al. [67]. Briefly, a double-pulsed frequency-double Nd: YAG (neodymium:yttrium aluminum garnet) laser, capable of generating a maximum of $0.3 \mathrm{~J} /$ pulse pair at a frequency of $1000 \mathrm{~Hz}$, was coupled with a high efficiency diffuser (Item No.: 1108417, Lavision, Gmbh, Goettingen, Germany). The laser with diffuser was positioned directly opposite a high speed CCD camera (Imager Pro PlusX, Lavision $\mathrm{GmbH}$, Goettingen, Germany) with a high magnification zoom lens (Navitar zoom 12x, 
Navitar Inc., Rochester, New York, USA) so that the axis of the diffuser and lens of the CCD camera were aligned. The abrasive jet flowed in a chamber between the diffuser and the lens of the CCD camera as shown in Fig. 4.1.

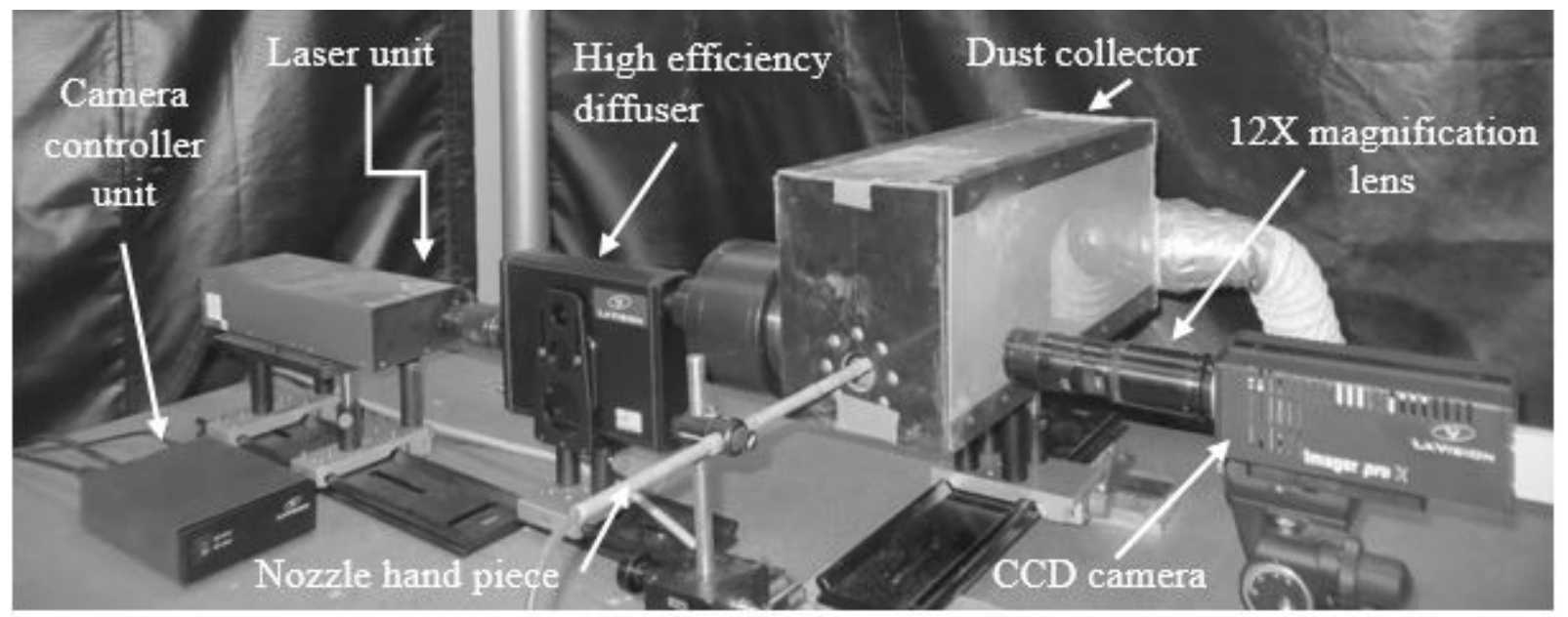

Fig. 4.1 Double-pulsed shadowgraphy apparatus.

The particle velocity distribution was measured from the image pairs using Davis Software (Lavision $\mathrm{GmbH}$, Goettingen, Germany). It was found that a pulse duration of $1 \mathrm{~ns}$ and time intervals of 1-3 $\mu$ s between the two pulses were suitable to capture the particle spatial distribution and measure the particle velocities for the given conditions. The radial and axial particle velocities at the nozzle exit were measured and set as input parameters for CFD modeling (Section 4.3). The coordinates of the particles at $0 \mathrm{~mm}$ (nozzle exit) and $20 \mathrm{~mm}$ away from the nozzle were recorded and used to determine the radial distribution of particles within the jet.

\subsubsection{CFD modeling}

CFD models in ANSYS Fluent 15.0 (ANSYS Inc., Cecil Township, PA, USA) were used to predict the abrasive particle trajectories for an air-particle jet surrounded by stationary air impinging a non-deforming target at perpendicular incidence. Fig. 4.2 shows the two-dimensional axisymmetric and three-dimensional domains used to model the impingement of the air jet on flat targets and curved rods having diameters between 3 and $5 \mathrm{~mm}$ (about 4-6.5 times the jet diameter). 
The realizable $\kappa-\varepsilon$ turbulence model was used to model the fluid, following the AJM modeling work of $\mathrm{Li}$ et al. [55]. For a given simulation, the same flow field was obtained using the $\kappa-\omega$ shear-stress turbulent transport (SST) model, but at slightly shorter convergence times. Li et al. [55] used two-way coupling and modeled particle-particle collisions for relatively large $27 \mu \mathrm{m}$ diameter particles. However, Crowe et al. [68] explained that particle volume fractions smaller than 0.001 can be treated with one-way coupling. Therefore, for the present volume fraction of $5.4 \times 10^{-8}$ particle-particle interaction was assumed negligible, and one-way coupling was used. The models converged with residuals below $10^{-3}$.

The $10 \mu \mathrm{m}$ particles described in Section 4.2 were given a shape factor of 0.76 as measured by Dehnadfar et al. [56], and were uniformly injected using the same discrete phase injection ratio settings described in Kowsari et al. [69] through the inlet. The particles were assigned initial axial and radial velocities of $195 \mathrm{~m} / \mathrm{s}$ and $0-3 \mathrm{~m} / \mathrm{s}$, respectively, to match those measured using shadowgraphy (Section 4.3.1). The particles were tracked using the Lagrangian discrete phase model. The flat target boundary was treated as a smooth, no-slip wall, and the other boundaries of the domain were treated as free with a pressure outlet condition. The surface roughness of as-received glass $\left(R_{r m s}=8 \mathrm{~nm}\right)$ was assumed to have a negligible effect on the rebound particle trajectories since the particles had much larger diameters than the local surface peaks and valleys. Although the mesh was refined near the target, the single-phase, air domains surrounding the jet were meshed using mostly $10 \mu \mathrm{m}$ quadrilateral elements. The impact velocities (both primary and secondary) of a particle released $100 \mu \mathrm{m}$ from the jet centerline differed by $6 \%$ for domains meshed with $20 \mu \mathrm{m}$ and $10 \mu \mathrm{m}$ elements. The difference in velocity for domains between 5 and $10 \mu \mathrm{m}$, however, was only $1 \%$, indicating convergence of the solution to a meshindependent state. To capture the shear flow in the viscous sub-layer near the targets, the dimensionless wall coordinate, $y^{+}$, was maintained below unity using near-wall grid refinement, as shown in Fig. 4.2. Both normal and tangential restitution coefficients were set to 0.2 as suggested by Slikkerveer and in't Veld [22] for similarly sized $\mathrm{Al}_{2} \mathrm{O}_{3}$ particles impacting glass. 


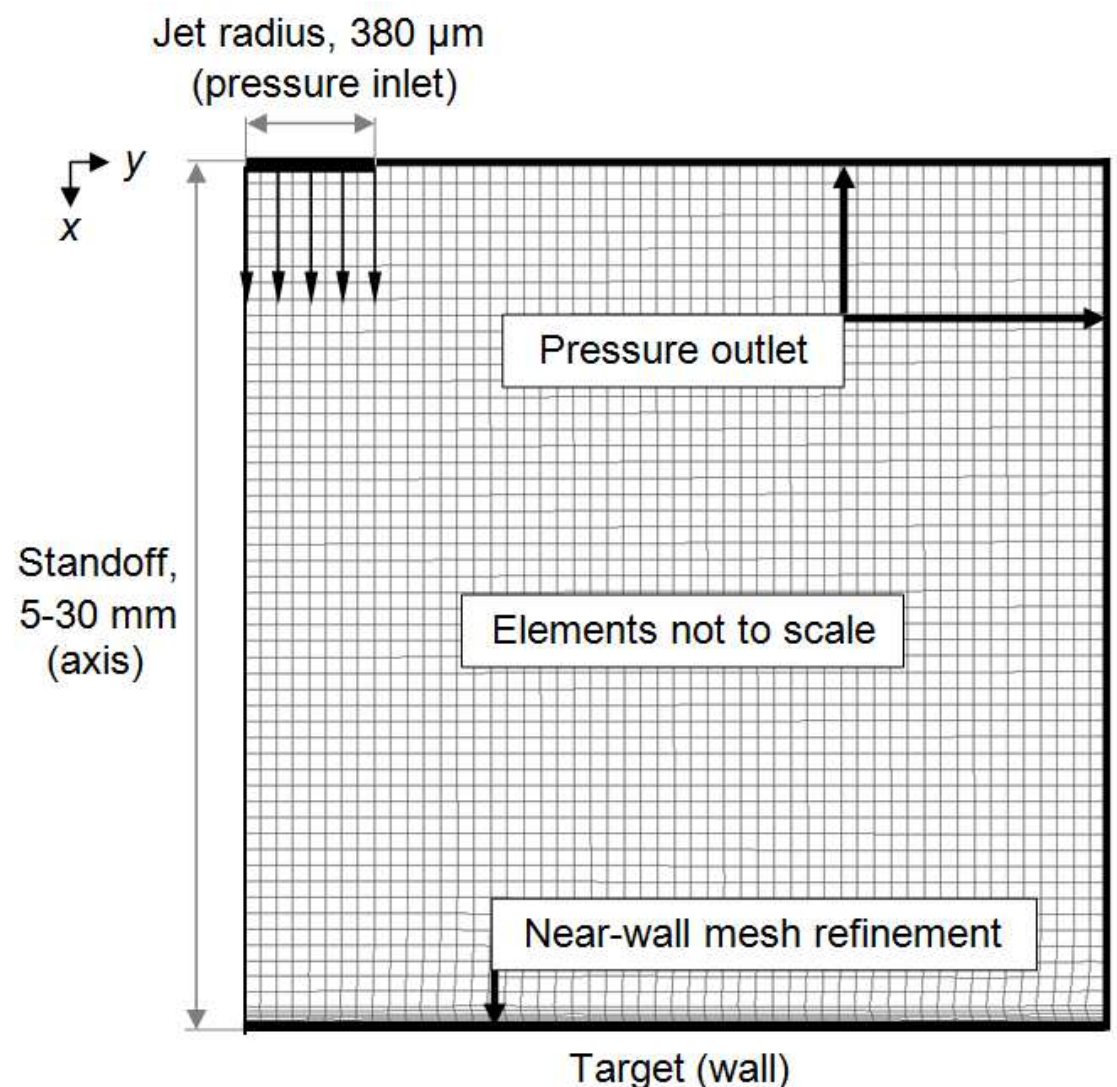

(a) 


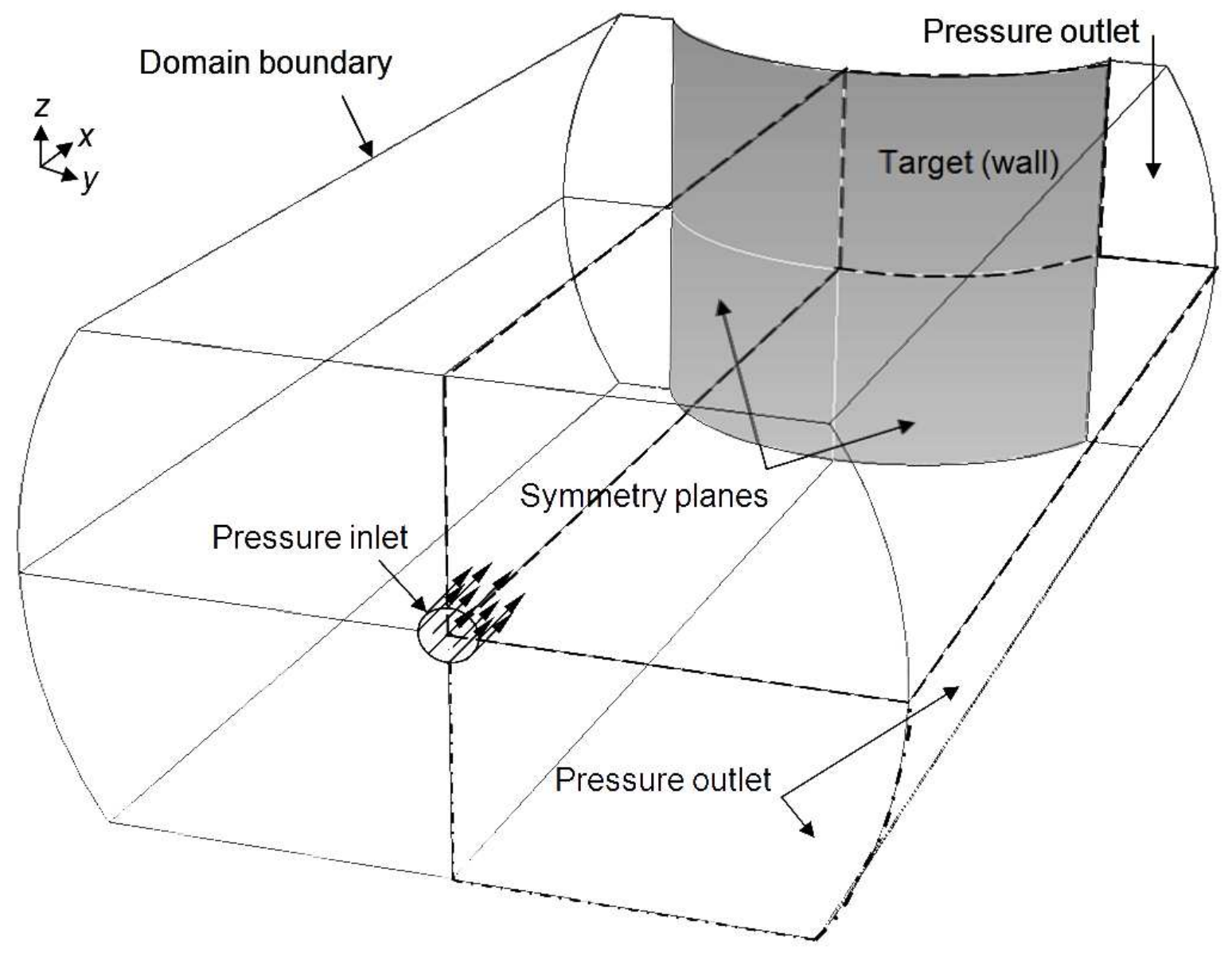

(b)

Fig. 4.2 Domains and boundary conditions of: (a) 2D axisymmetric CFD model of the impingement of an air-particle jet on a flat target and (b) 3D CFD model of the impingement of an air-particle jet on a curved target.

\subsection{Results and discussion}

\subsubsection{AJM jet structure}

Fig. 4.3a shows that the particles exited the nozzle with a relatively small divergence angle. This is also evident in Fig. 4.4a which shows the particle distribution obtained by analyzing shadowgraphic images of the abrasive jet plume in a region between the nozzle exit and $5 \mathrm{~mm}$ downstream of it. These data imply that the jet plume diameter at the nozzle exit was about $1 \mathrm{~mm}$ 
(Fig. 4.4a), and that the divergence angle was about $1.5^{\circ}$ from the jet axis. There was good agreement between the size of the primary plume measured on paper after $30 \mathrm{~s}$ exposure to the abrasive jet (2.8 $\mathrm{mm}$ diameter at $20 \mathrm{~mm}$ standoff) and the one obtained from shadowgraphy (3.1 $\mathrm{mm}$ at $20 \mathrm{~mm}$ standoff). As explained in Section 4.2.2, the axial and radial particle velocity components obtained from these shadowgraphy measurements were used in the CFD model to produce the same divergence, as shown in Fig. 4.3b. The relatively small divergences in the present work, measured both optically and numerically, are consistent with the findings of Shipway and Hutchings [57] for nozzles having smooth inner walls so that particle scattering due to wall collisions was relatively small.

The results of the shadowgraphy experiments in Fig. 4.4b, showed that approximately $90 \%$ of the particles were within $1.55 \mathrm{~mm}$ of the centerline, at a standoff distance of $20 \mathrm{~mm}$. The relatively small dose of particles outside this region did not significantly affect the erosive footprint, in part because they had relatively small velocities. This was confirmed by conducting experiments on paper targets at a standoff of $20 \mathrm{~mm}$ while varying the exposure time. It was found that the size of the footprint remained constant for up to $30 \mathrm{~s}$, but increased by about $20 \%$ after 5 min. In summary, approximately $90 \%$ of the particles were found in an approximately conical primary plume of about $3 \mathrm{~mm}$ diameter at a $20 \mathrm{~mm}$ standoff. The stray particles outside of this primary plume did not significantly affect the erosive footprint, since they produced appreciable erosion only after relatively long exposure times ( $30 \mathrm{~s})$. In comparison, when machining microchannels a given point on a target is exposed to the jet for about $5 \mathrm{~s}$ at a typical scan speed of 0.5 $\mathrm{mm} / \mathrm{s}$. 
Back-lit

Nozzle

Jet of particles

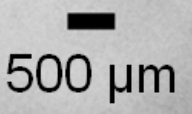

Side-lit

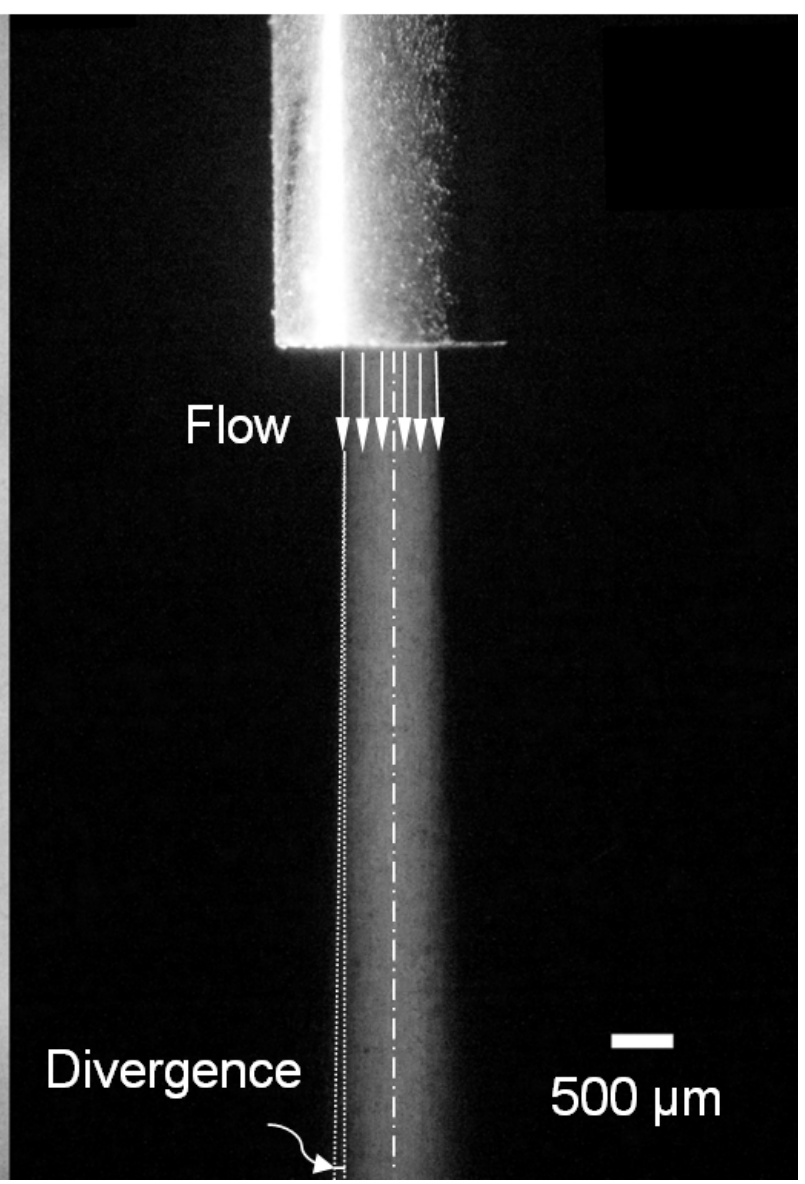

(a) 
Air velocity magnitude $(\mathrm{m} / \mathrm{s})$

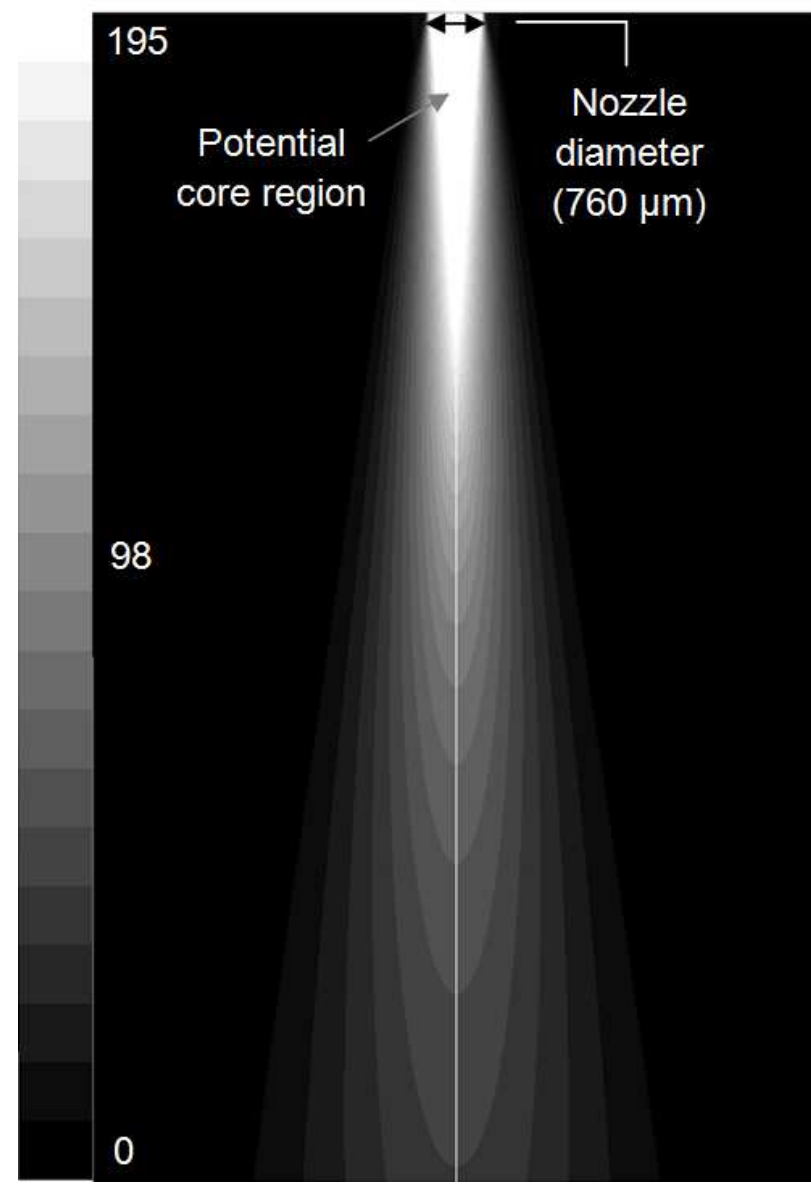

$10 \mu \mathrm{m}$ particle velocity magnitude $(\mathrm{m} / \mathrm{s})$

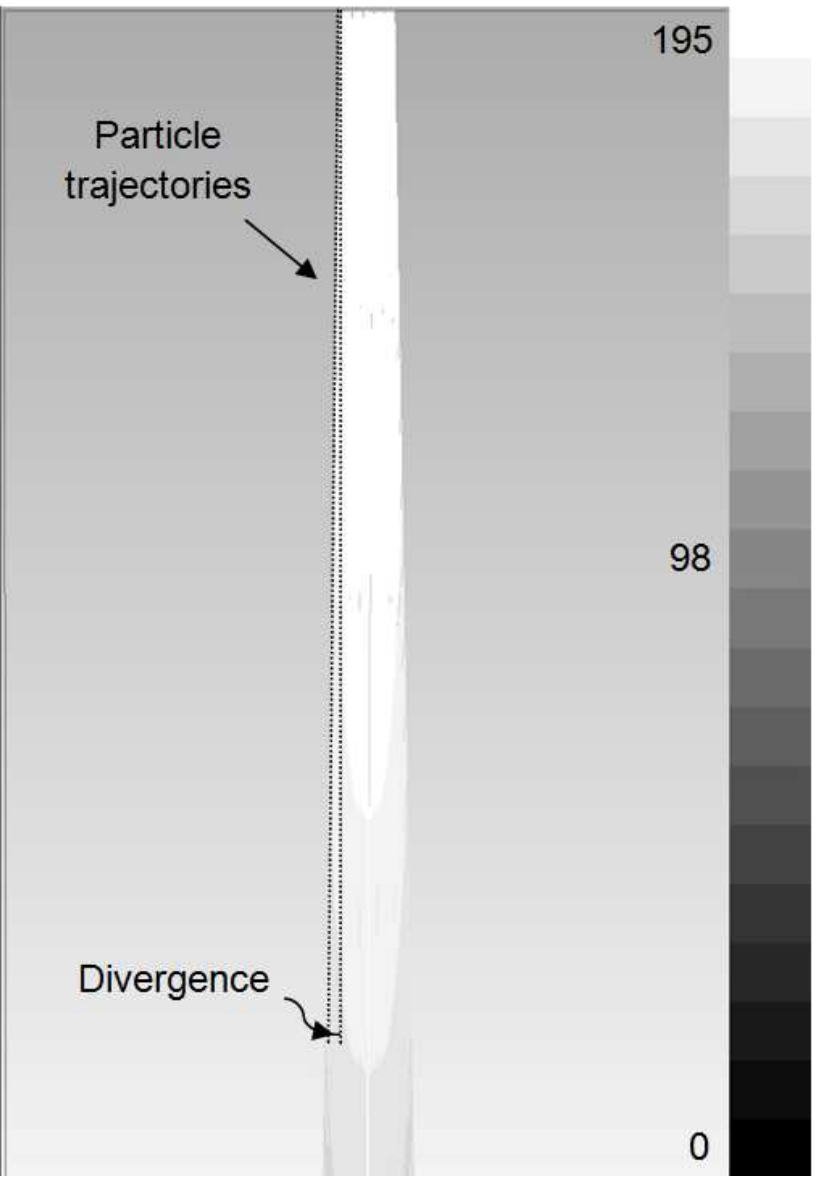

(b)

Fig. 4.3 AJM jet. (a) Microscope images of the AJM jet. (b) Air and particle velocity magnitude contours obtained using CFD. 

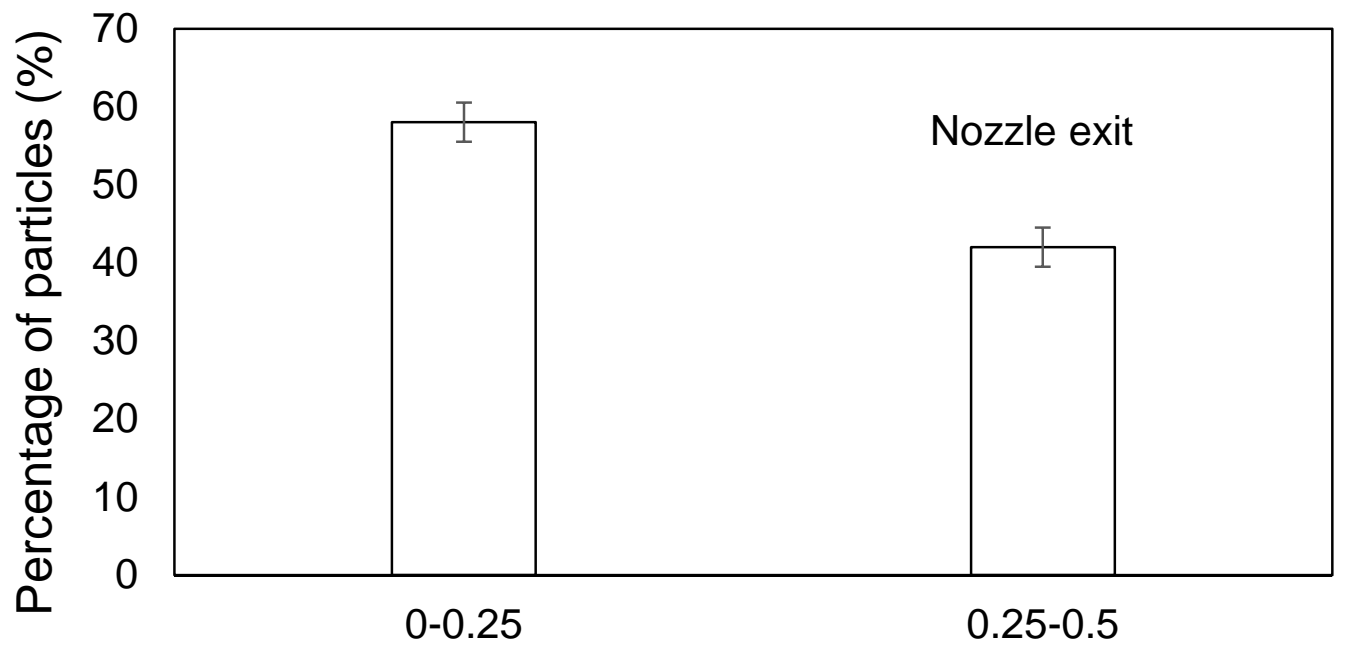

Radial distance from jet axis $(\mathrm{mm})$

(a)

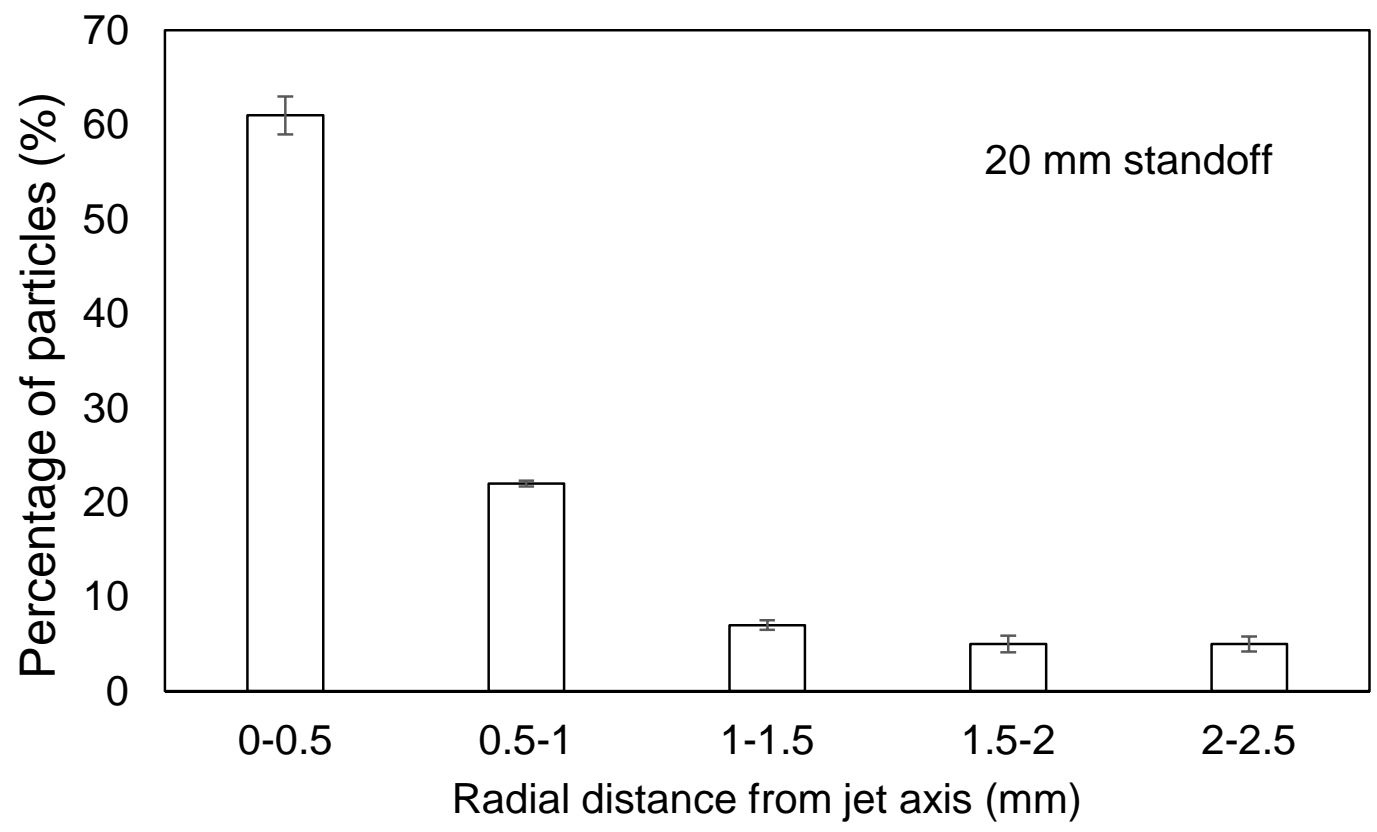

(b)

Fig. 4.4 Radial distribution of particles within the jet: (a) at nozzle exit (b) at $20 \mathrm{~mm}$ standoff, obtained from shadowgraphy. The error bars indicate the standard deviations obtained from three measurements of approximately 15000 particles each. 


\subsubsection{Erosive footprint prediction for flat surfaces}

Fig. 4.5a shows the CFD-predicted flow field and particle trajectories near a target at a standoff of $5 \mathrm{~mm}$. It is seen that particles within the primary plume travel in straight lines from the nozzle until they impact the target, and did not follow the curvature in the air streamlines near the surface. As explained by Kowsari et al. [65] for a similar AJM jet, the momentum equilibration number, $\lambda$, was approximately 900 as calculated using

$$
\lambda=\frac{\rho_{p}\left(d_{p}\right)^{2} v_{j e t}}{18 \mu\left(d_{n}\right)}
$$

where $\rho_{p}$ is the particle density, $d_{p}$, is the particle diameter, $v_{j e t}$ is the jet velocity, $\mu$, is the dynamic viscosity of air, and $d_{n}$ is the nozzle diameter. As explained by Humphrey [70], values of $\lambda>>1$ indicate that the particles in the air jet are unlikely to follow the fluid streamlines. Upon initial impact, Fig. 4.5a shows that, depending on their radial position, the particles rebounded to various heights to impact a second time farther away at angles between about $30-70^{\circ}$ to the surface. Fig. 4.5a-Fig. 4.5d show that the rebound height increased with increasing standoff distance despite the decrease in the incident velocity at larger standoffs. This was because the air velocities at the maximum rebound heights also decreased with increasing standoff, thus the particles experienced less resistance in their rebound trajectories and rebounded to greater heights. This was further-investigated using an energy balance on a rebounding particle described as

$$
\frac{m V_{r}^{2}}{2}-m g h_{\text {apex }}=\int_{0}^{h_{\text {appex }}} F_{d} \cdot d x
$$

where $m$ is particle mass, $V_{r}$ is rebound velocity, $g$ is the gravitational acceleration, $h_{\text {apex }}$ is the maximum rebound height, $F_{d}$ is the particle drag force, and $x$ is the vertical distance from the surface. In order to estimate $V_{r}$, a coefficient of restitution of 0.2 was assumed, although this choice was not critical since the main objective was a relative comparison of the effect of standoff distance. Using the CFD predictions of the incident velocity and $h_{\text {apex }}$, Fig. $4.5 \mathrm{e}$ shows that the particle drag loss computed using the left-hand side of Eq. (4.2) did indeed decrease with increasing standoff distance, explaining why $h_{\text {apex }}$ increased. Fig. $4.5 \mathrm{e}$ also shows that these drag 
loss values were consistent with those obtained from the integration of the particle drag force, $F_{d}$ , shown in Fig. 4.5f, as a function of $x$ as calculated using the CFD model. The drag coefficient was obtained from Haider and Levenspiel [71] as

$$
C_{d}=\frac{24}{\operatorname{Re}}\left(1+b_{1} \mathrm{Re}^{b_{2}}\right)+\frac{b_{3} \mathrm{Re}}{b_{4}+\mathrm{Re}}
$$

where the coefficients $b_{1}-b_{4}$ are functions of the ratio of the surface area of an equivalent sphere to the actual particle surface area, which was set to 0.76 for the $10 \mu \mathrm{m}$ nominal diameter alumina abrasives as measured by Dehnadfar et al. [56] .

It is seen in Fig. 4.5 that the higher rebounds at larger standoffs caused the second-strikes to occur farther away from the centerline which enlarged the erosive footprint. Moreover, the average second-strike impact angles increased with increasing standoff even as the footprint became larger; i.e. $72^{\circ}, 77^{\circ}, 82^{\circ}$, and $83^{\circ}$ for $5 \mathrm{~mm}, 10 \mathrm{~mm}, 20 \mathrm{~mm}$, and $30 \mathrm{~mm}$ standoffs, respectively, as evident in Fig. 4.5a, 4. 5b, 4. 5c, and 4. 5d. This was because the stagnation zone and the associated lateral flow of air was smaller at larger standoff distances so that second-strike particles experienced less deflection immediately before impact. For example, Fig. 4.5a shows that the lateral air velocity near the surface in the $y$-direction caused the second-strike particles to deflect and impact at shallower angles. This lateral flow did not significantly enlarge the footprint on its own. 
$5 \mathrm{~mm}$ standoff

Air velocity magnitude $(\mathrm{m} / \mathrm{s})$

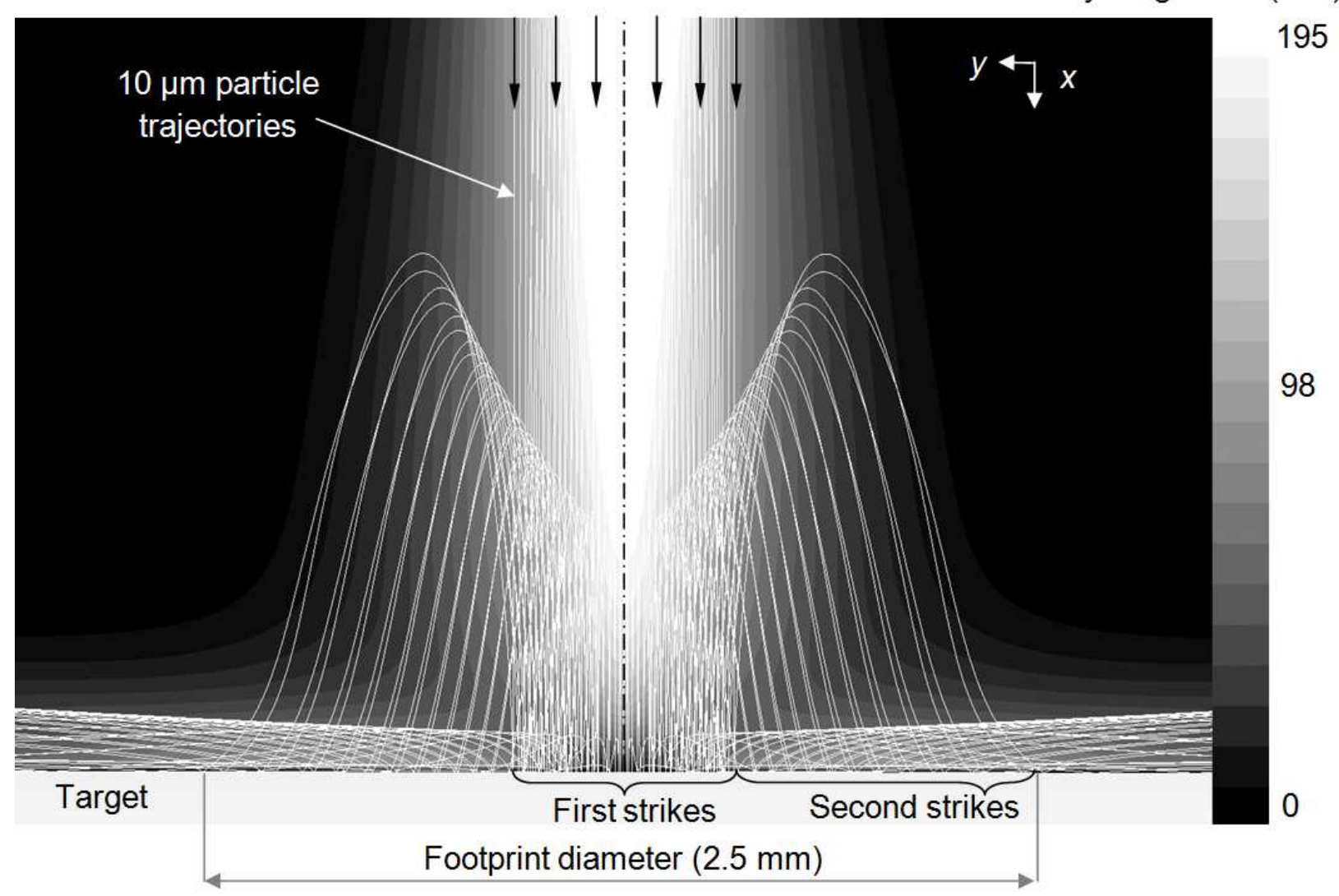

(a) 


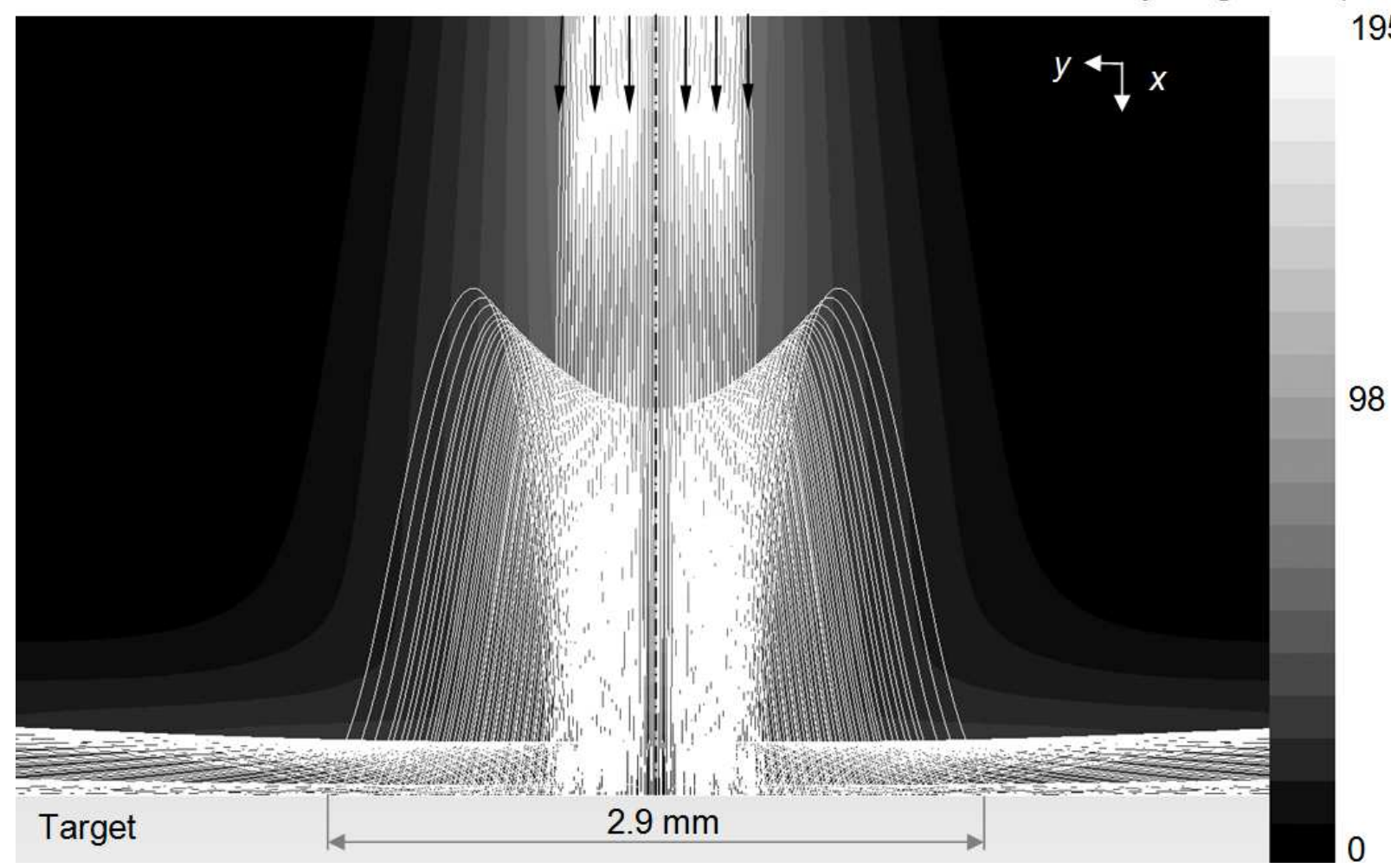

(b)

$20 \mathrm{~mm}$ standoff

Air velocity magnitude $(\mathrm{m} / \mathrm{s})$

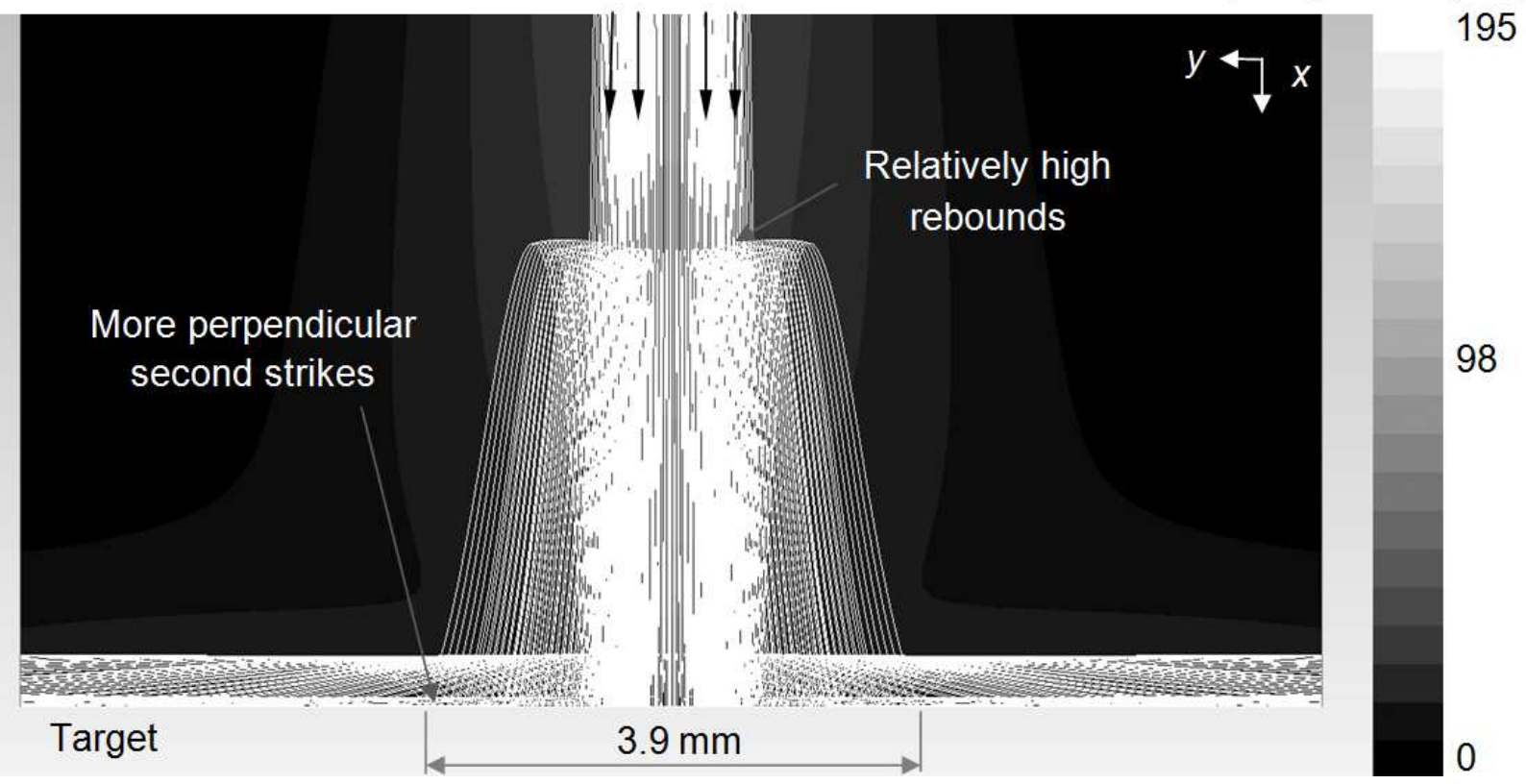

(c) 

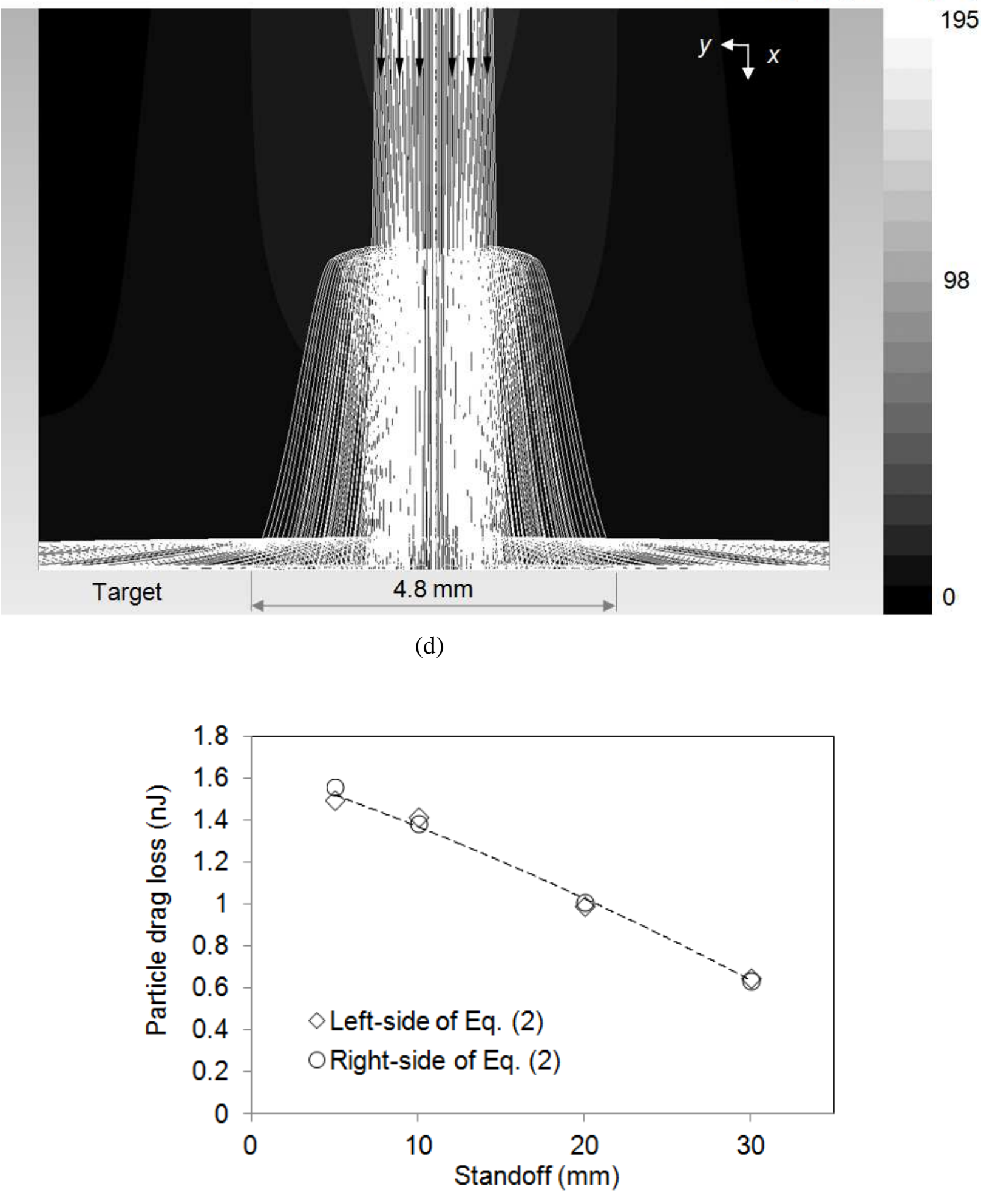

(e) 


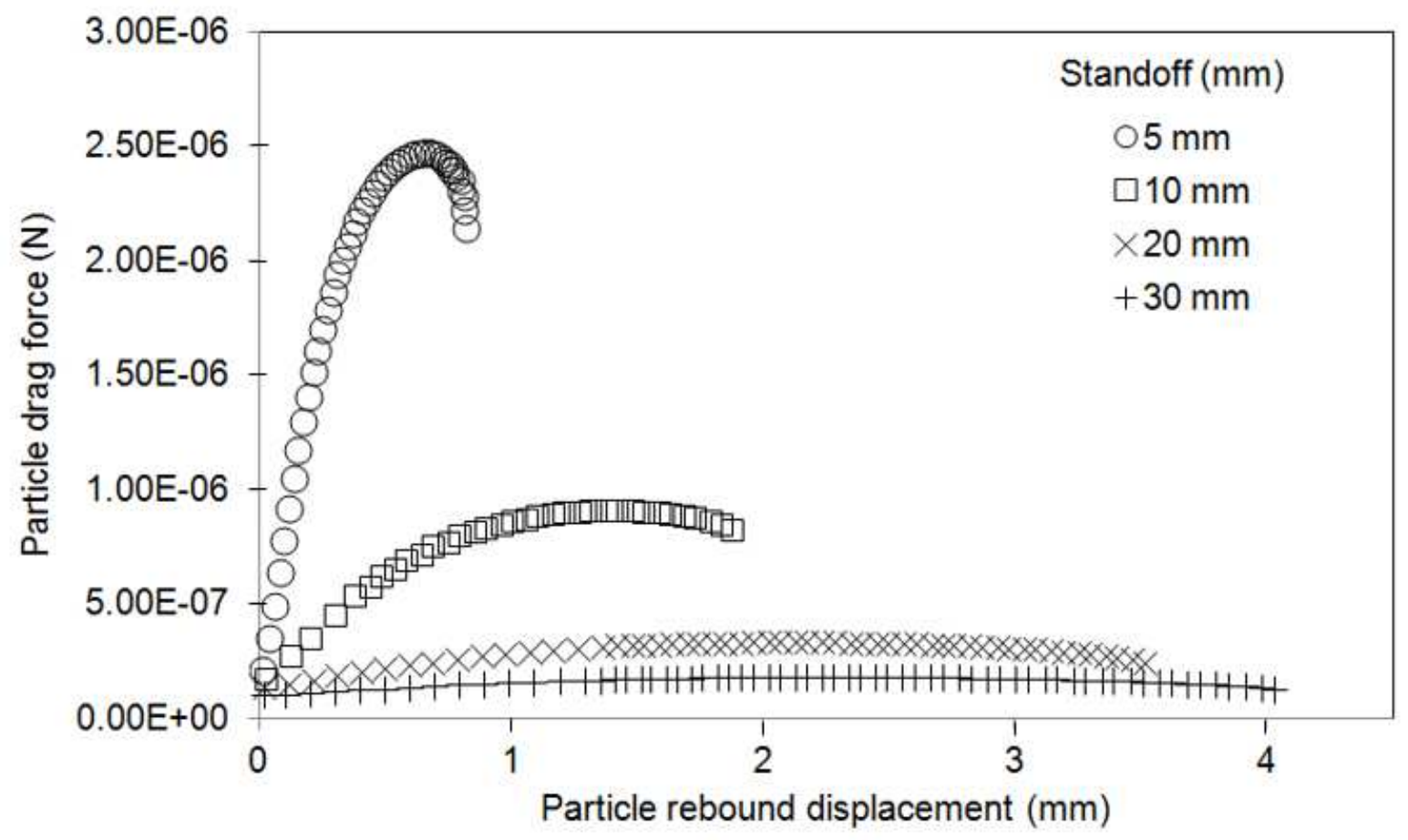

(f)

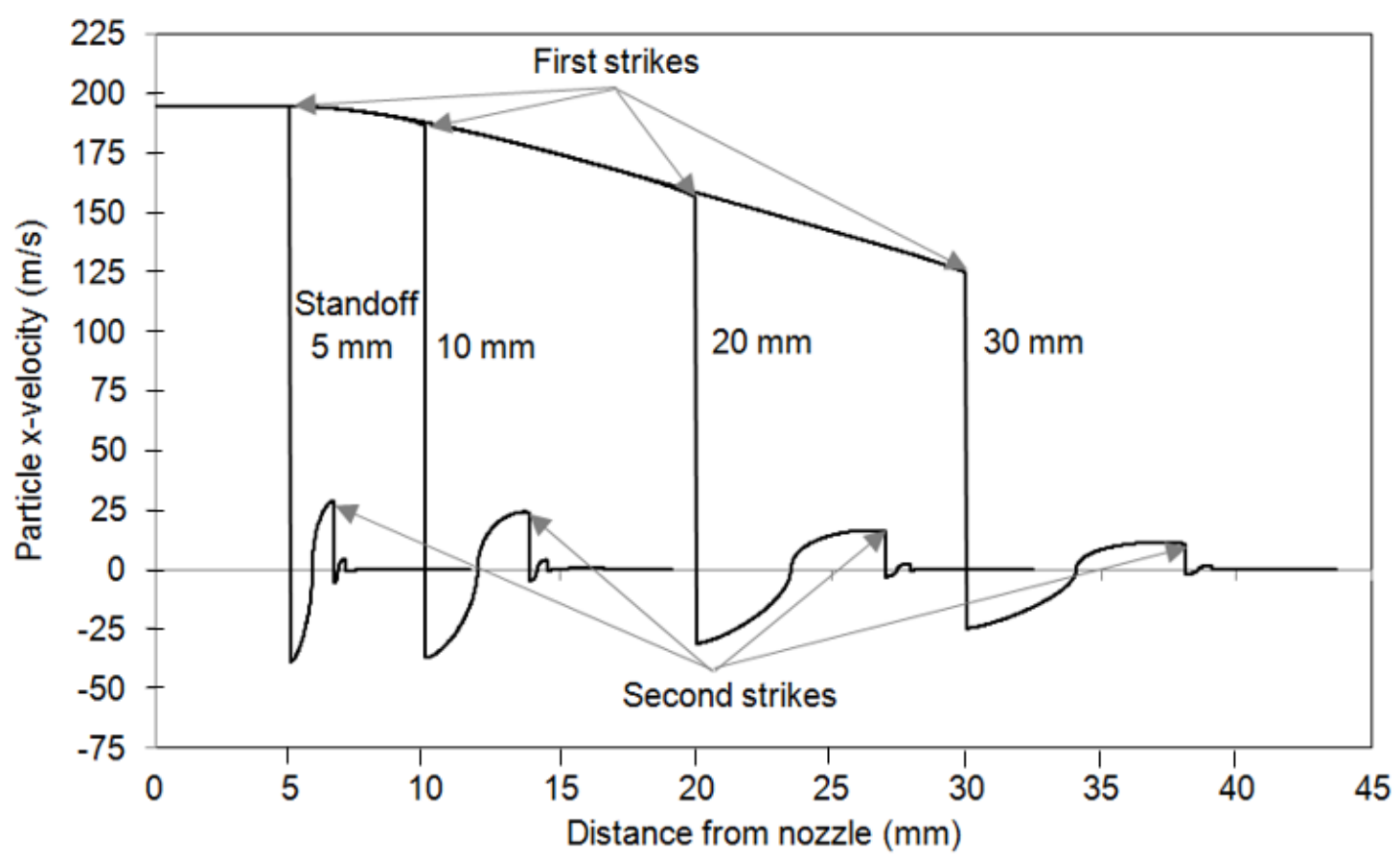

(g) 
Fig. 4.5 Impingement of AJM jets on flat targets. Air velocity magnitude contours and $10 \mu \mathrm{m}$ diameter particle trajectories for standoff distances of: (a) $5 \mathrm{~mm}$, (b) $10 \mathrm{~mm}$, (c) $20 \mathrm{~mm}$, and (d) $30 \mathrm{~mm}$. (e) Drag energy loss as a function of standoff distance for particles released at the nozzle centerline obtained from either $h_{\text {apex }}$ and $V_{r}$ or direct integration of $F_{d}$ (Eq. (4.2)). (f) CFD prediction of particle drag force versus particle rebound displacement for different standoffs. (g)

Axial velocities of particles released from a given mesh element at the inlet boundary approximately $100 \mu \mathrm{m}$ from the nozzle centerline (13\% of the nozzle diameter) at various distances from the target using the models of Fig. 4.5.

The centerline rebound height at the $20 \mathrm{~mm}$ standoff was 5.1 times that in the $5 \mathrm{~mm}$ case. At $20 \mathrm{~mm}$ standoff, the $42 \mathrm{~m} / \mathrm{s}$ flow velocity at the apex peak of a rebounding centerline particle was sufficient to re-accelerate the particles to impact the target a second time with a velocity of approximately $18 \mathrm{~m} / \mathrm{s}$. Fig. $4.5 \mathrm{~g}$ shows the axial velocity of particles released from a mesh element about $100 \mu \mathrm{m}$ from the jet centerline (13\% of the nozzle diameter) for the 5-30 $\mathrm{mm}$ standoff CFD simulations. It is seen that both the primary impact velocity, the maximum rebound velocity (negative), and the second-strike velocity all decreased with increasing standoff distance. This same trend was evident at all distances from the nozzle centerline. For a given standoff distance, the maximum variation in the first and second strike velocities between particles released at different nozzle radii was about $20 \%$.

Wensink and Elwenspoek [72] explained that the ductile-brittle transition occurred at 17 $\mathrm{nJ}$ for borosilicate glass. Although the secondary impact kinetic energies (0.1-0.6 nJ) are lower than this threshold value for brittle cracking, the values are sufficient to cause ductile erosion in the glass targets as in the work of Nouraei et al. [58] in which the impact velocities of similarlysized alumina particles ranged between $20-60 \mathrm{~m} / \mathrm{s}$ corresponding to kinetic energies of 0.4-3.7 $\mathrm{nJ}$.

\subsubsection{Experimental validation}

The CFD simulations revealed that the total erosion at all standoffs consisted of the contributions of the first strikes near the center of the jet and the second impacts in an outer ring as shown in Fig. 4.5. The first-strike footprints enlarged with increasing standoff due to the divergence of the primary plume, as observed in Fig. 4.6a. The second-strike footprints also grew 
with increasing standoff since the drag loss of rebounding particles was greater at smaller standoffs (Fig. 4.5e). These predictions were compared to the experimentally-measured AJM footprints on glass at standoffs between 5-30 mm. Fig. 4.6a shows good agreement between the predicted and measured results for the net footprints including both the first and second strikes. It is hypothesized that the slight over-estimations of the CFD-obtained second-strike footprint diameters were due to particle fragmentation during the first strike which decreased the rebound height and the size of the rebounding particles, thereby reducing the measured footprint size in the glass targets, as explained by Hadavi et al. [73]. The results suggested that the effective AJM footprint is characterized by the superposition of two cone-shaped plumes for first and second strike erosion, as illustrated schematically in Fig. 4.6b. While the divergence angle of the primary plume, $\alpha$, is governed only by the jet divergence, the second-strike angle, $\psi$, defining the boundary of the second-strike plume, depends on the rebound conditions. For example, it is hypothesized that $\psi$ increases with increasing coefficient of restitution, and thus the numerical jet footprint prediction methodology could serve as a tool to predict restitution coefficients for various abrasive-target combinations.

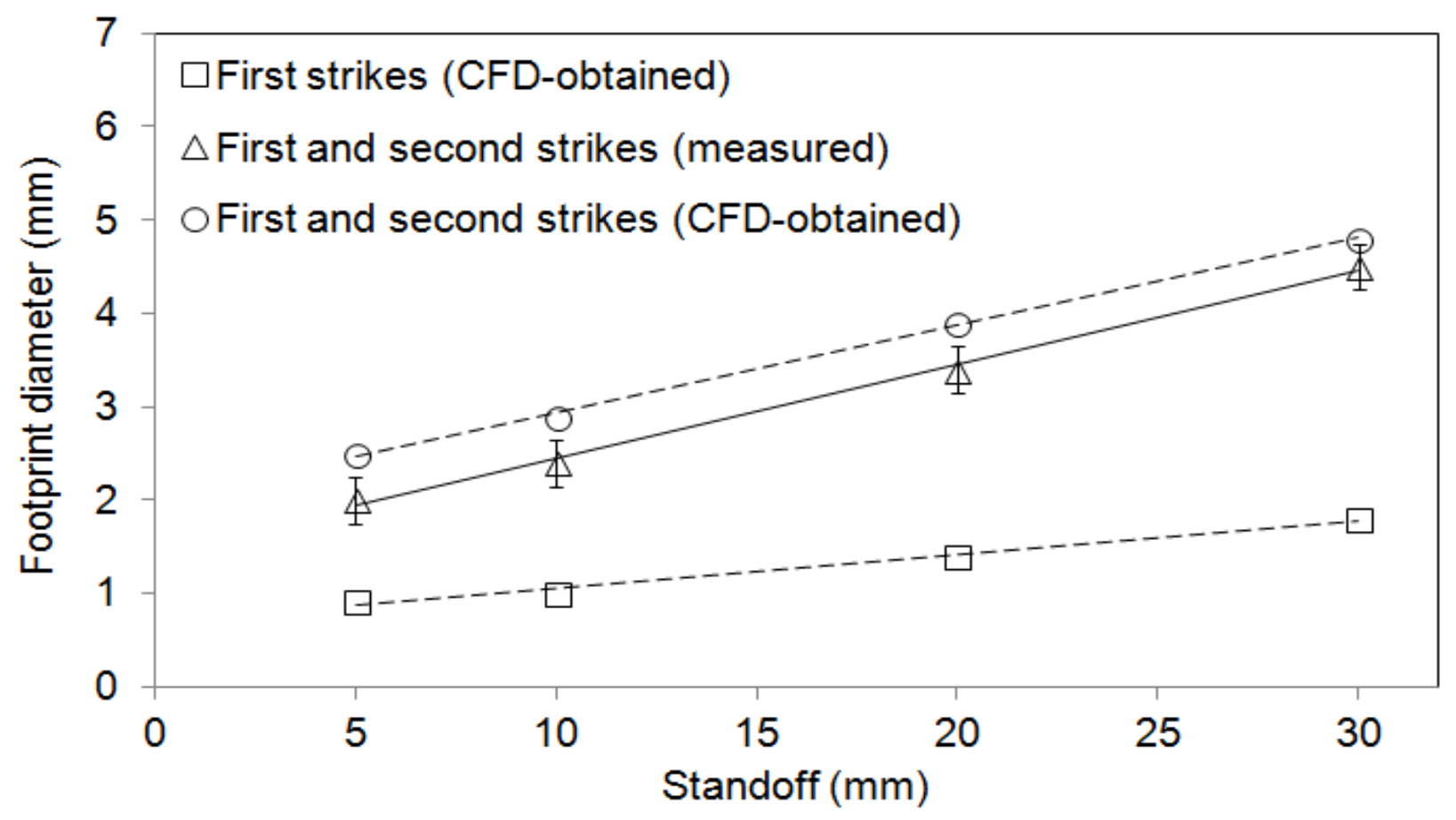

(a) 


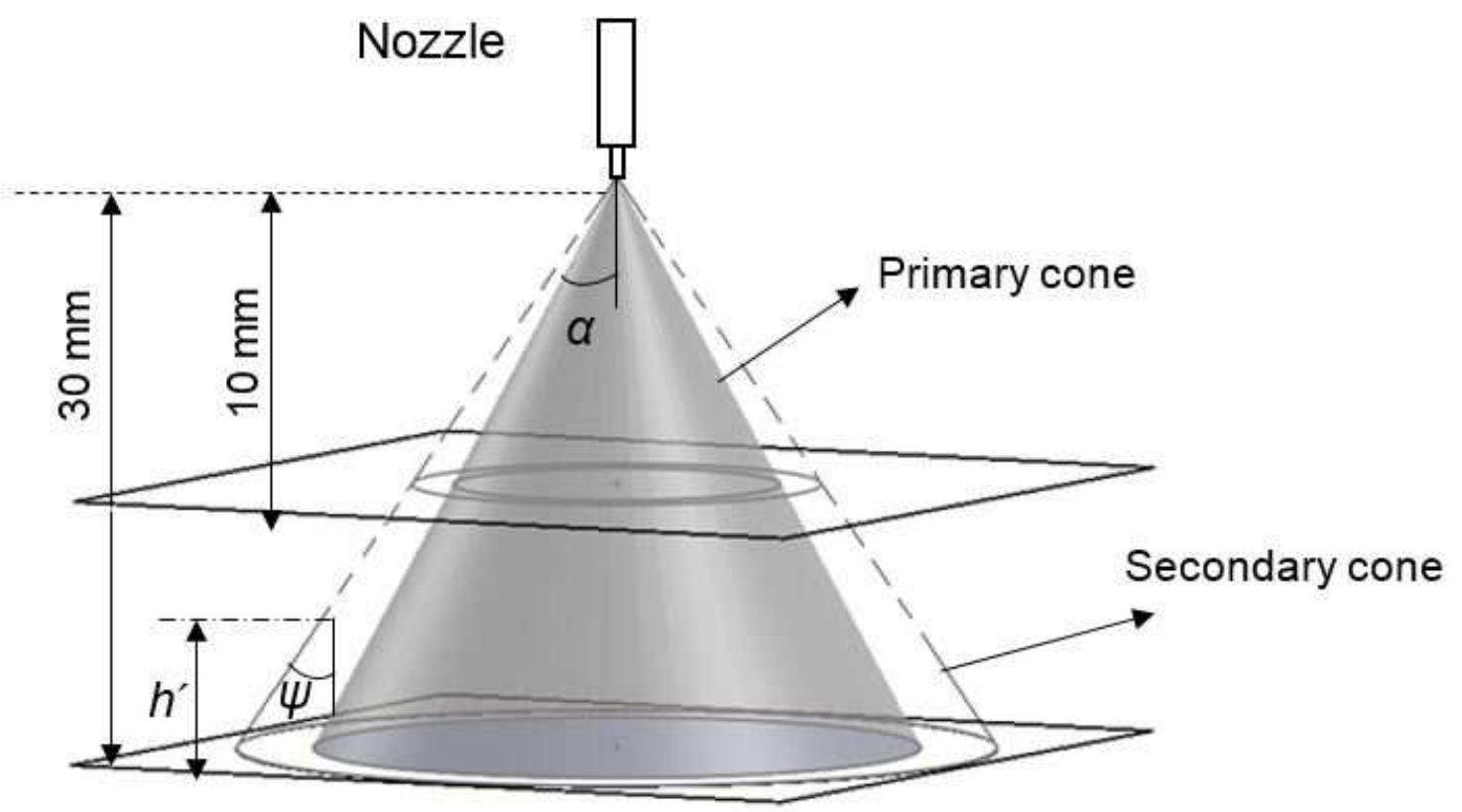

(b)

Fig. 4.6 (a) Predicted (dashed lines) and measured (solid lines) erosive footprint diameter versus standoff with and without secondary particle impacts. The lines are to guide the eye only. Error bars represent \pm 1 standard deviation for 3 measurements. (b) Schematic representation of intersections of primary and secondary plumes with successive target planes at standoffs of 10 $\mathrm{mm}$ and $30 \mathrm{~mm} . \psi$ defines the second-strike cone angle, and $h^{\prime}$ is the apex height of a corresponding particle after rebound from the target.

\subsubsection{Erosive footprint prediction for curved surfaces}

Nouhi et al. (2016) showed that the erosive efficacy inferred from the measurement of an eroded footprint on a flat surface could not be used to predict the footprint on a curved surface. It was therefore of interest to determine whether the CFD model could be used to do this. The erosive footprints of an AJM jet from a $460 \mu \mathrm{m}$ diameter nozzle (200 kPa, $10 \mu \mathrm{m}$ aluminum oxide particles, as in Nouhi et al. [66] ) on 3 and $5 \mathrm{~mm}$ rods were predicted using computational domains similar to that shown in Fig. 4.2(b). Fig. 4.7 presents the CFD-predicted air velocity magnitude 
contours and particle trajectories at a standoff of $10 \mathrm{~mm}$. The curvature of the targets caused the first strike impact angles to vary, which significantly affected the degree of lateral rebound leading to second strikes. For a given particle within the air jet, the local normal of the first strike, $\theta$, was larger for a $5 \mathrm{~mm}$-diameter rod than for the $3 \mathrm{~mm}$ rod, $\varphi$, thereby widening the net erosive footprint with increasing target curvature. However, despite the footprint enlargement, the number of rebounds without second strikes also increased since a larger number of particles deflected beyond the edges of the rod, as seen in Fig. 4.7b, thus reducing the dose of secondary impacts. In summary, differences in target curvature can strongly affect the particle impact trajectories in both the primary and secondary plumes.

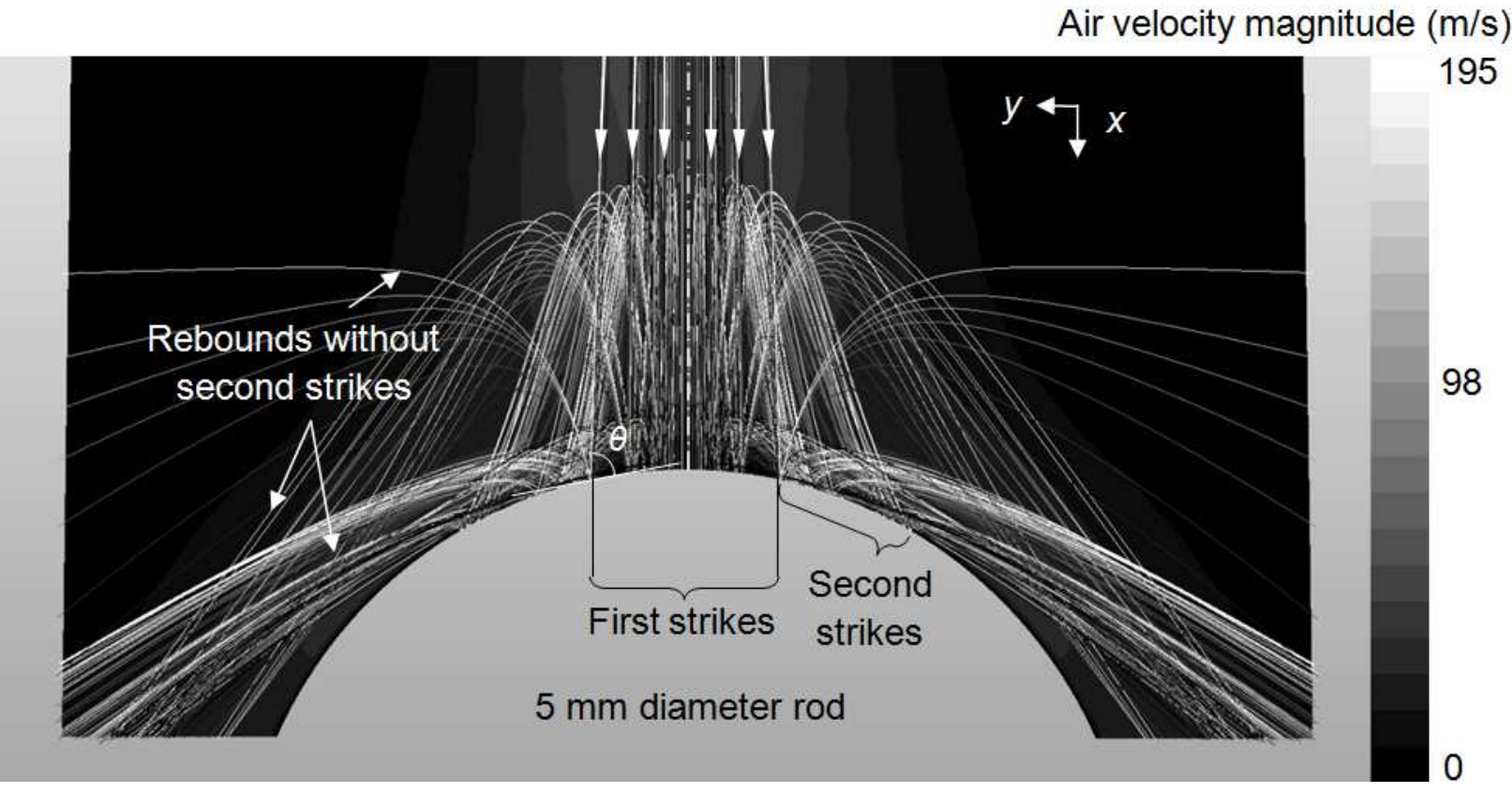

(a) 


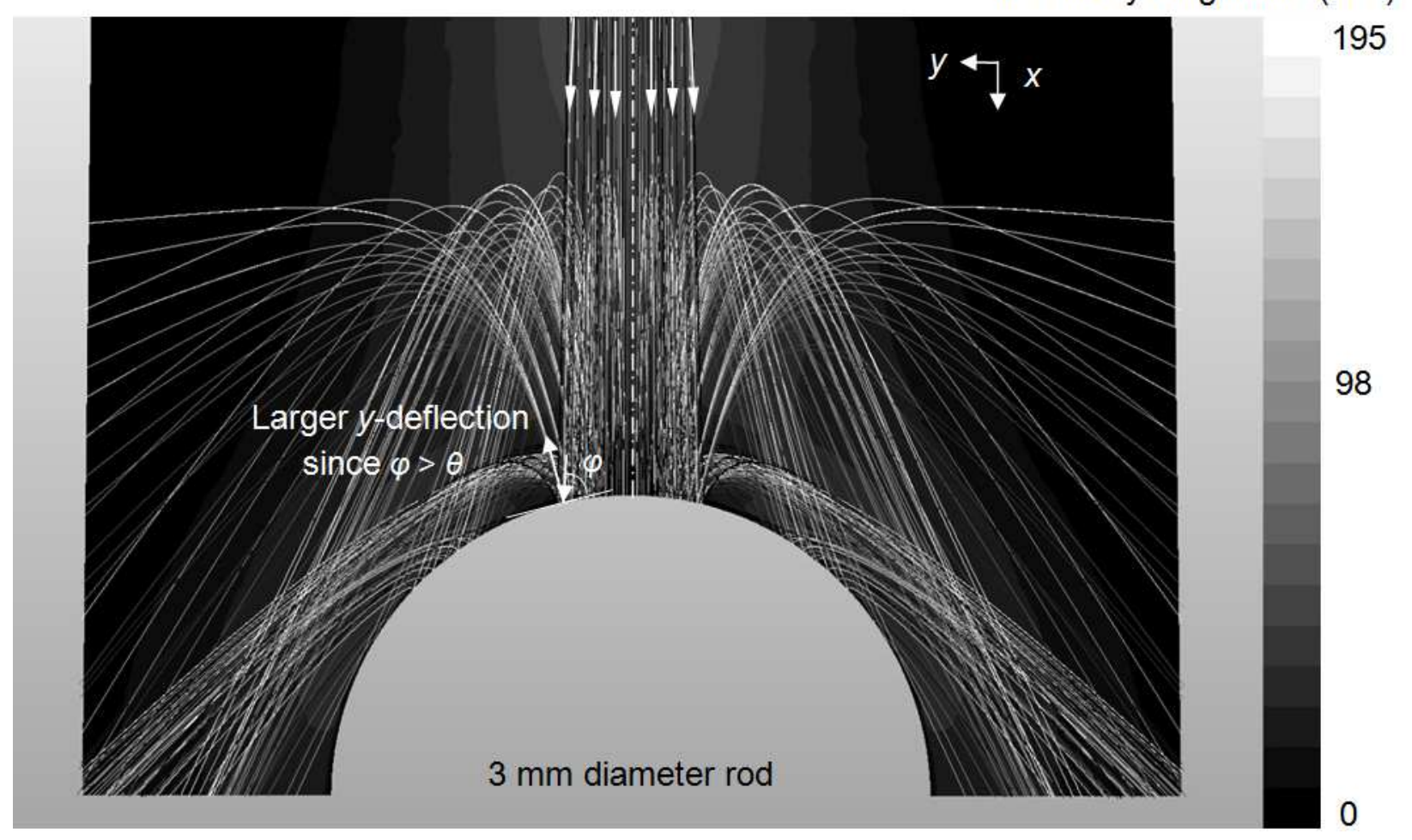

(b)

Fig. 4.7 Impingement of AJM jets on curved targets at a standoff of $20 \mathrm{~mm}$. Air velocity magnitude contours and particle trajectories for rod diameters of: (a) $5 \mathrm{~mm}$, and (b) $3 \mathrm{~mm}$.

The net effect of the differences in particle trajectories brought about by target curvature was determined by predicting the distribution of erosive efficacies on a flat glass surface and a 5 mm glass rod using erosion maps produced by CFD as described in Kowsari et al. [65] . Briefly, the measured dependence of erosion on particle impact angle and impact velocity were defined in the erosion model of ANSYS Fluent to obtain the three-dimensional erosion maps shown in Fig. 4.8. These maps reflected the net erosion produced by both the primary and secondary plumes. The maps were then converted to the two-dimensional erosion patterns across a machined channel that would result from a nozzle scan by summing the erosion rates along lines parallel to the scan direction across the footprint. 


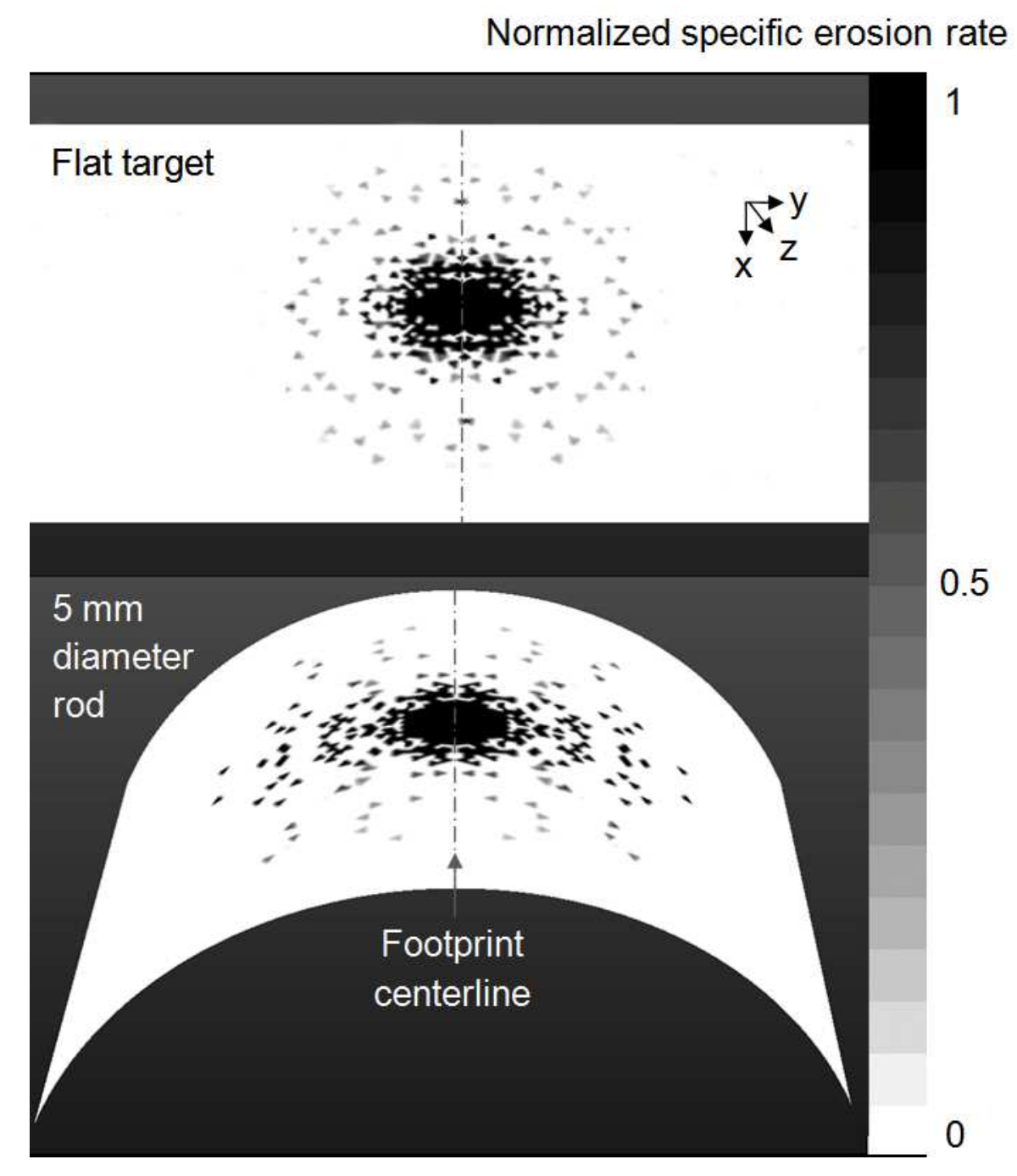

Fig. 4.8 CFD-obtained normalized erosion maps on a flat target and a $5 \mathrm{~mm}$ diameter rod. Each map was normalized by its maximum specific erosion rate (mass eroded per unit mass of erodent).

The resulting erosive efficacy distribution was then fit to a Weibull-type function

$$
\delta\left(\frac{\beta}{x}\right)^{2} e^{-\left(\frac{\beta}{x} y\right)^{2}}
$$

where $y$ is the transverse coordinate along the channel width, $x$ is the vertical coordinate measured from the nozzle tip to the target surface (Fig. 4.9) and $\beta$ is an effective nozzle focus coefficient that reflects both first and second strikes. 


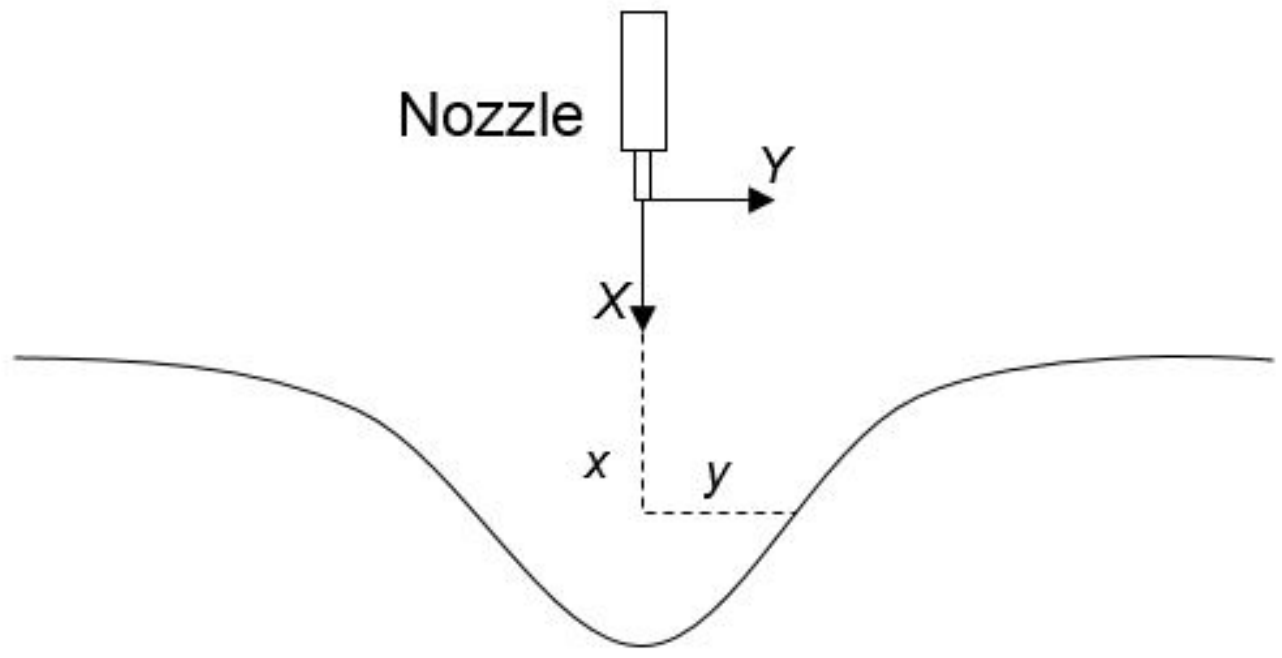

Fig. 4.9 A schematic of Weibull-type function describing the shallow eroded profile. The coordinates $(y, x)$ of a typical point on the profile are shown.

The $\beta$ values for both the flat and curved surface cases were then inferred from curve-fitting a Weibull distribution to the normalized erosion patterns to obtain Fig. 4.10. Although the curves of Fig. 4.10 were obtained from the superposition of two different plumes, one due to primary and the other due to secondary impact, their sum created a single smooth erosive efficacy curve. The predicted values of $\beta, 31$ on flat target and 24 on $5 \mathrm{~mm}$ diameter glass rod, were in good agreement ( $10 \%$ difference) with the measured ones, 28 and 22 on flat and rod targets, respectively, given by Nouhi et al. [66]. 


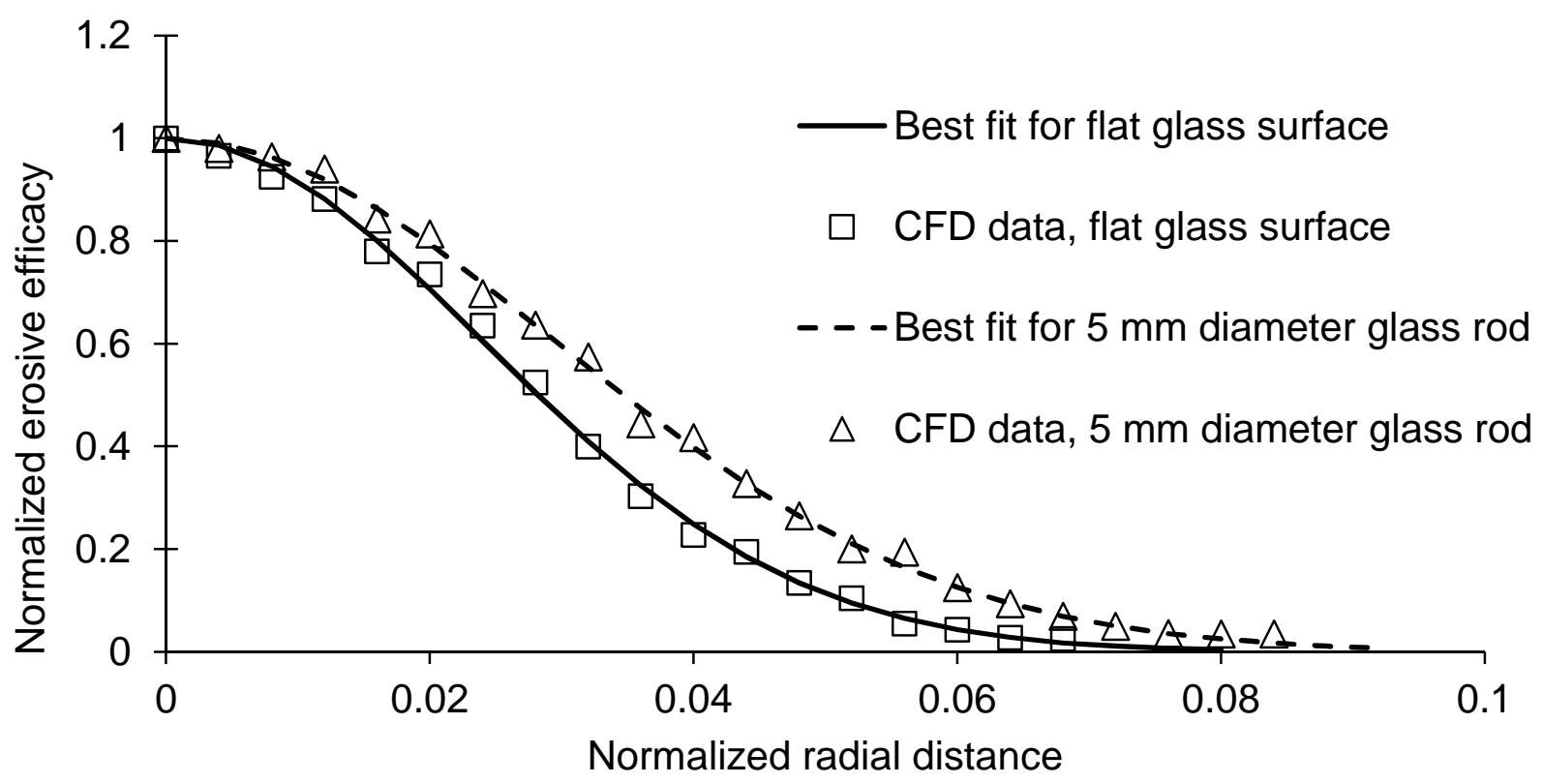

Fig. 4.10 Normalized erosive efficacies and the best fits (Weibull distribution) for flat and curved ( $5 \mathrm{~mm}$ diameter) glass targets. The abscissa was normalized by standoff distance and the ordinate was normalized by the depth of the channel centerline.

\subsection{Implications for AJM}

Ghobeity et al. [9] and Getu et al. [74] showed that a shallow channel profile which was machined by a scanning nozzle on flat target at $90^{\circ}$ incidence could be used to characterize the erosive efficacy delivered to both ductile and brittle surfaces. They also showed that when implemented in an appropriate surface evolution model, the erosive efficacy inferred from the shallow profile could be used to successfully predict the shape of micro-channels machined using AJM. On flat surfaces, the erosive efficacy determined from a shallow profile includes both the primary and secondary particle strikes on the flat surface, and therefore the Weibull distribution obtained in this manner can be used to predict the evolution of machined surface profiles. This footprint on flat targets can be viewed as being at the intersection of a single effective particle cone and the target (Fig. 4.6b). However, the results of the present work confirm the hypothesis of Nouhi et al. [66] that erosion due to second strikes is more pronounced when the initial target surface 
curvature is higher, consequently making the shallow channels machined on flat targets inappropriate for directly characterizing the erosive efficacy on rods or other curved targets, where the effective cone and footprint will be a function of curvature.

In general, the effective value of $\beta$ for a given target curvature can be determined either by CFD modeling to obtain the erosion maps as in Section 4.3.3, or an effective value can be obtained by adjusting $\beta$ in the surface evolution model to fit the measured shallow first-pass profile of a channel machined on the curved target (Nouhi et al. [66] ). Measurements and modeling have shown that $\beta$ is sensitive to the target curvature and must be adjusted if, for example, a rotating rod is being machined using AJM as a lathe. For instance, according to Nouhi et al. [66], $\beta$ had to be decreased about $9 \%$, from 22 to 20 , when the rod diameter was decreased (curvature increased) from $5 \mathrm{~mm}$ to $3 \mathrm{~mm}$. For relatively deep channels in curved targets, the increasing slope of the local surface geometry with increasing channel depth would likely alter the erosive pattern. Therefore, additional CFD modeling would be required beyond the first pass, analogous to what was done in Kowsari et al. [65] for abrasive slurry jet micro-machining.

\subsection{Summary}

A computational fluid dynamics (CFD)-aided procedure was presented for the prediction of the erosive footprints resulting from abrasive jet machining (AJM) of both flat and curved targets. The divergence of an AJM jet was measured using laser-pulsed shadowgraphy and by blasting holes through paper. Using these results together with CFD models, it was found that the net erosive efficacy footprint on a surface was the result of the superposition of two approximately conical erodent plumes; a primary one leading to first strikes and a secondary one reflecting second particle impacts. CFD modeling showed that approximately $90 \%$ of the particles travelled within the primary plume, with the remaining $10 \%$ at the periphery being so sparse that they did not affect the footprint. On flat targets, the particle incident velocities, the air velocities, and the rebound particle drag losses were found to decrease with increasing standoff distance. These effects caused an increase in the particle rebound heights after their first strike and a broadening of their trajectories such that the net footprint of first- and second-strike particles was enlarged, but the average impact angles decreased with increasing standoff. The predicted kinetic energies of particles striking a second time were large enough to erode glass targets. 
The erosive footprint was also found to depend on target curvature, because the local slope changed the angle at which the particles rebounded, thus changing the distribution of second strikes to the surface. The presented methodology provided fundamental understanding of air-driven particle erosive footprints that is needed in modeling of curved surfaces. In such cases, the footprint size would depend on the local surface slope that changes with increasing feature depth, thereby requiring further CFD modeling beyond those for shallow features, analogous to the approach taken in Kowsari et al. [65] for abrasive slurry jet micro-machining.

In Chapter 5, a model will be proposed which implements the erosive efficacy and nozzle focus coefficient, $\beta$ determined using the techniques of this chapter to predict the material removal during machining of rotating glass and PMMA rods. 


\section{Chapter 5 Abrasive jet turning of glass and PMMA rods and the micro- machining of helical channels}

This chapter is based on a paper which was submitted.

A. Nouhi, J.K. Spelt, M. Papini, "Abrasive jet turning of glass and PMMA rods and the micromachining of helical channels", submitted to the Journal of Micromechanics and Microengineering

\subsection{Introduction:}

The micro-machining of small curved devices made of brittle materials, such as glass and ceramics, and ductile materials like polymers and metals is required in optical and biomedical equipment [1,2]. Matsumura and Ueki [32] used a ball-end mill to make $20 \mu \mathrm{m}$ deep grooves on $6 \mathrm{~mm}$ diameter cylindrical glass rods. The milling tool was tilted in the feed direction (along the cylinder axis) to improve the surface finish and it was observed that the axial feed speed had to be kept below $0.24 \mathrm{~mm} / \mathrm{s}$ to avoid cracking of the glass. Furutani et al. [1] developed a lathe for glass rods using electro-chemical discharge machining. They machined grooves up to $0.6 \mathrm{~mm}$ deep and $0.8 \mathrm{~mm}$ wide in a $5 \mathrm{~mm}$ diameter glass rod, and found that the depth, width and roughness of the machined channels increased with increasing applied voltage, and that increasing the rotational speed did not affect the machined depth, but resulted in a decrease in width and roughness.

Gottmann et al. [75] produced micro-structures in glass by in-volume selective laserinduced etching which is a two-step process where the glass workpiece was first subjected to a pulsed laser in the portions to be patterned. The glass was then exposed to an aqueous solution of potassium hydroxide $(\mathrm{KOH})$ which selectively etched the laser modified regions of the surface. The technique was demonstrated by producing a $50 \times 50$ array of $73 \mu \mathrm{m}$ diameter holes in $1 \mathrm{~mm}$ thick glass, and by producing $1 \mathrm{~mm}$ diameter cylindrical gears.

Degawa et al. [76] presented a laser fabrication method for making miniature internal threads of S0.5 standard in a $1.0 \mathrm{~mm}$ thick glass substrates; i.e. major diameter of $0.5 \mathrm{~mm}$ with a pitch of $0.125 \mathrm{~mm}$. The drawbacks of this method were its long processing time (32 min for 7 threads with a pitch of $125 \mu \mathrm{m}$ ), and the ridges, grooves and chipping that resulted on the glass surface. 
Abrasive water jets (AWJ) have been studied widely as lathes. For example, Li et al. [33] studied the AWJ turning process on $59 \mathrm{~mm}$ diameter steel rods, finding that the depth of cut increased with increasing water pressure and turning speed, and decreasing axial feed speed and jet angle relative to the surface. A dimensional analysis was performed to estimate the depth of cut. Similarly, Zhong and Han [34], investigated the effect of AWJ process parameters in the turning of glass, and found that the surface finish was improved using a lower pressure and axial feed speed and a higher turning speed. Manu and Babu [35] implemented Finnie's theory of erosion to model the material removal from a $25.4 \mathrm{~mm}$, rotating 6063-T6 aluminum alloy rod using abrasive waterjet turning with the nozzle displaced radially from the rod axis so that the erosion was tangential, focusing at the edge of the rod. The variation of the particle impact angle due to the instantaneous rod curvature during machining was considered in the model. However, the model neglected the particle spread in the jet, resulting in an error of about 10\%. Carach et al. [36] used a tangential abrasive waterjet (AWJ) as a lathe to machine Incoloy alloy 925, an alloy that is difficult to machine conventionally because of rapid tool wear. It was observed that higher traverse speeds (jet footprint movement parallel to the rod axis) resulted in a higher roughness, but the effect was relatively small.

Abrasive jet micro-machining (AJM) uses high-speed air jets to accelerate abrasive particles toward a workpiece surface. Like AWJ it is capable of cutting and milling many materials without excessive forces or thermal damage, which is a significant advantage over many other machining technologies [1]. In addition, AJM has a low capital and operating cost, does not involve hazardous materials and does not require a clean room [51]. Sogabe et al. [77] developed a microblasting technique to machine patterns on cylindrical parts such as bearings. Three materials were tested: brass and two carbon steel alloys (SS400 and S45C). The workpiece was covered with a 75 $\mu \mathrm{m}$ thick patterned PVC mask film and the target specimen was blasted in a lathe operation. The depth and width of machined grooves were $6 \mu \mathrm{m}$ and $270 \mu \mathrm{m}$, respectively. They observed that material removal increased with increasing pressure and increasing transverse offset of the jet axis from the part axis, while increasing the nozzle standoff decreased it. The roughness increased with increasing pressure, but it was approximately independent of nozzle standoff and offset distance. Nouhi et al. [66] machined straight axial channels in glass and PMMA rods of different radii and presented a model to predict the channel profile that considered the variation of nozzle standoff and the spread in particle trajectories across the channel width. The use of AJM as a lathe and as 
a means of machining helical grooves in rods has not been previously investigated to our knowledge.

In inertial microfluidic channel flow, it is known that liquid mixing and particle concentration within a liquid are influenced by the curvature of the channel longitudinal axis. For example, if channels in a flat plate are curved instead of being straight, the velocity difference between the center of the channel and near the walls results in a secondary flow (termed a Dean flow) that can facilitate fluid mixing [78]. Liu et al. [79] found that mixing in serpentine planar channels (150 $\mu \mathrm{m}$ deep and $300 \mu \mathrm{m}$ wide) was impaired by the counteracting effect of each subsequent turn in the opposite direction. In contrast, the three-dimensional curvature of helical channels improved mixing.

Sudarsan and Ugaz [80] investigated the efficiency of fluid mixing in five different compact spiral-shaped channels, each $150 \mu \mathrm{m}$ wide and $29 \mu \mathrm{m}$ deep. Over a wide range of Reynolds numbers (0.02-18.6) it was observed that diffusion was the main mechanism for mixing at low velocities and so mixing improved with channel length, while at higher flow rates the Dean effect had a dominant effect. It was also noted that an abrupt increase in the channel cross-sectional area enhanced mixing due to the creation of an expansion vortex.

Manlapaz and Churchill [81] studied the effect of pitch (separation of spiral loops) on the Dean flow in tubes that were helically wound with a range of ratios of coil radius to tube radius beginning at 0.1 . They found that the effect of pitch was significant only when it was greater than the radius of the spiral.

Lee et al. [82] successfully separated bacteria based on their size using $250 \mu \mathrm{m}$ deep helical micro-channels fabricated using Somos resin (DSM N.V.) by laser sterolithography. The channels had a trapezoidal cross-section, an outer radius of $1 \mathrm{~mm}$ and a pitch of $1 \mathrm{~mm}$. Besides exploiting the Dean flow, the central sample stream was focused using an outer sheath of liquid injected along the walls of the channel. Helical micro-channels were preferred over planar spiral ones since they had a constant radius of curvature and hence a constant Dean flow. This simplified the flow behavior and provided greater control of the separation process. However, stereolithography is a laser-based technology which is more complex and costly than AJM, and is mostly limited to fabrication in polymers.

The present work had two objectives related to the use of AJM as a lathe for the machining of glass, plastic and metal rods. Firstly, experiments were used validate a model of the material 
removal process for rods of arbitrary diameter. Secondly, a novel AJM masking technique, using helical steel springs, was developed to make microfluidic channels of controlled depth and width. The AJM lathe model was used to predict the channel shapes and rate of material removal.

\subsection{Experiments}

\subsubsection{AJM apparatus}

All experiments were conducted using an AccuFlo AF10 Micro-Abrasive Blaster, (Comco, Inc. Burbank, CA, USA). The operating air pressure was kept at $200 \mathrm{kPa}$. A refrigeration air dryer a dehumidifier, and a desiccant filter were used to minimize the moisture in the inlet air to the powder reservoir which was fitted with a rotary electric mixer to minimize powder bed compaction and particle agglomeration. The operating procedures described in [45] were used to further enhance the repeatability of the experiments. Aluminum oxide $\left(\mathrm{Al}_{2} \mathrm{O}_{3}\right)$ powder (Comco, Inc. Burbank, CA, USA) with a mean diameter of $11.4 \mu \mathrm{m}$ (log-normal distribution with standard deviation of $3.27 \mu \mathrm{m}$ ) was used in all experiments.

\subsubsection{AJM lathe}

Fig. 5.1 shows a schematic of the AJM lathe which was constructed using a DC motor (Cytron, 12 V, Gear Motor/Encoder, Robot shop Inc., Mirabel, Quebec, Canada). The speed was controlled by a driver and computer up to a maximum of $1000 \mathrm{rpm}$. The lathe apparatus of Fig. 5.1 was clamped to a computer-controlled linear stage with a positioning resolution of $0.5 \mu \mathrm{m}$ for feed control. The rods were held by a chuck (corrosion-resistant Jacobs drill chuck, Apex Tool Canada LP) and had a wobble deflection (peak to peak) of less than $150 \mu \mathrm{m}$ measured $80 \mathrm{~mm}$ from the chuck. This was considered to have a negligible effect on the machined patterns since the standoff distance was $100 \mathrm{~mm}$ and the footprint of the erosive particles had a diameter of $2 \mathrm{~mm}$. 


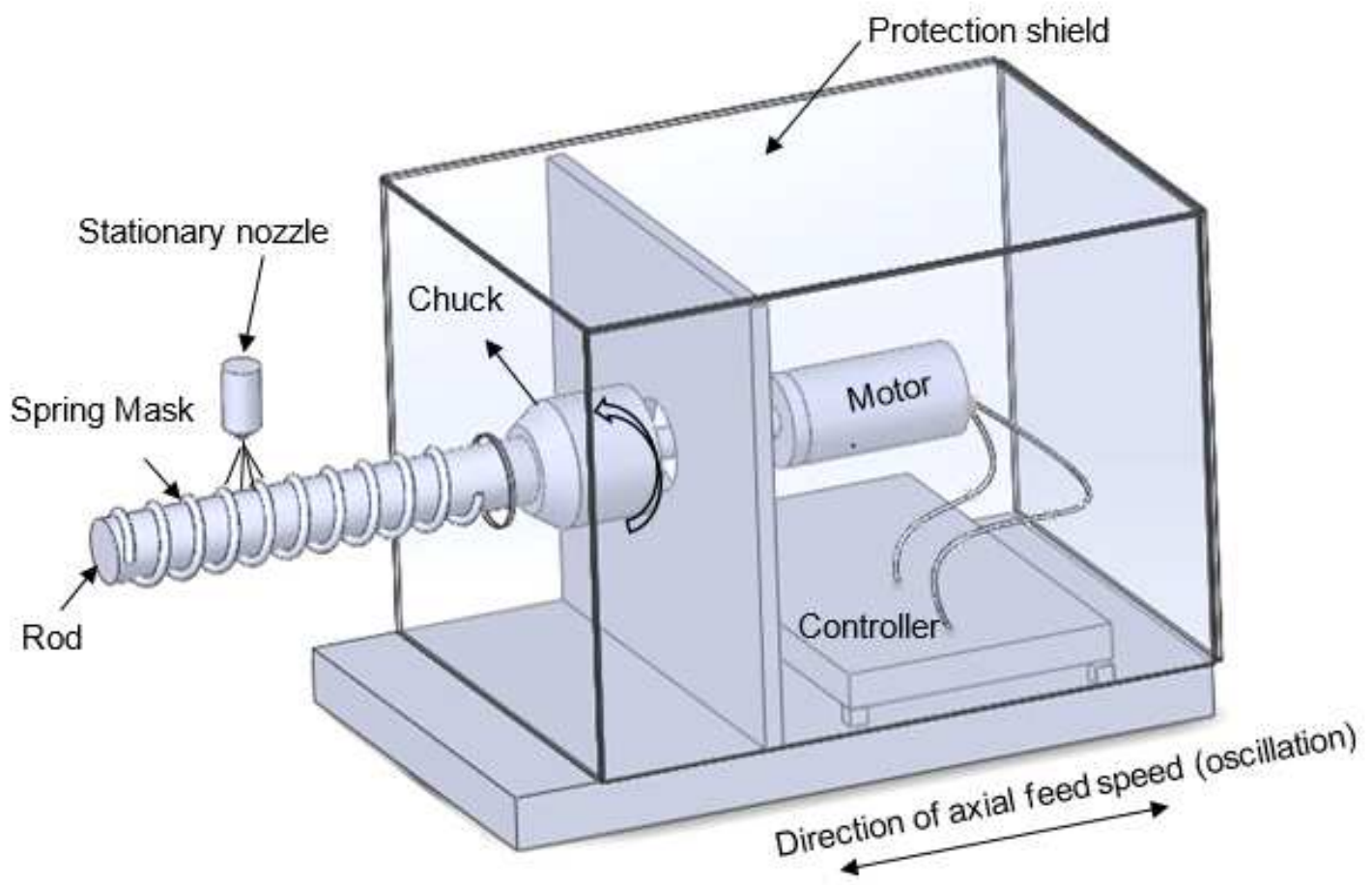

(a)

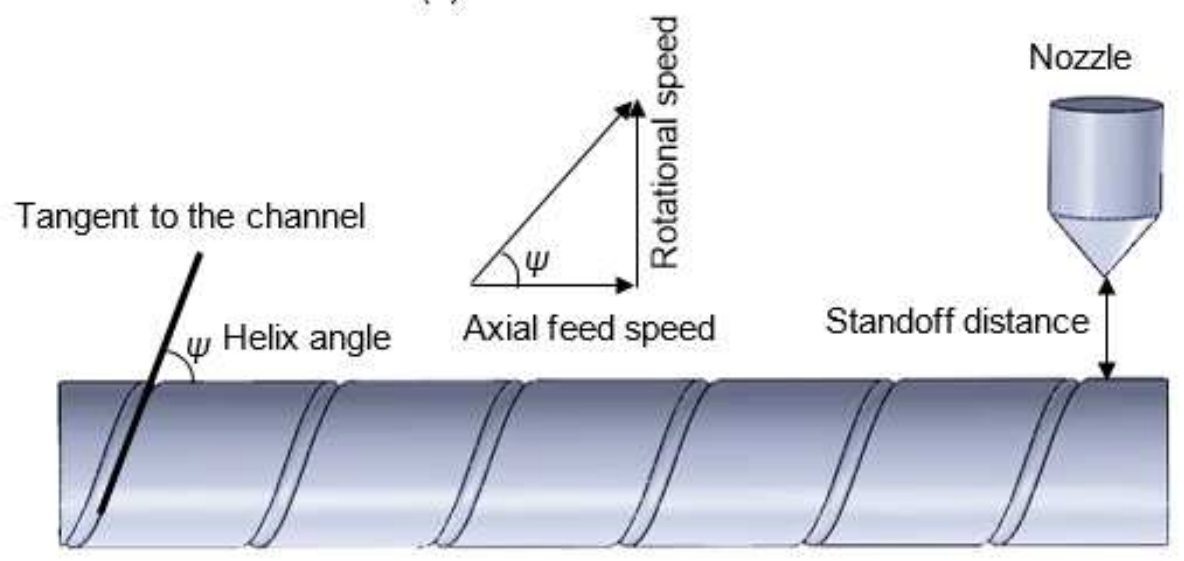

(b)

Fig. 5.1 a) AJM lathe, b) a schematic of a helical channel machined in one nozzle pass. 


\subsubsection{Unmasked lathe machining}

The machining was performed on a $5 \mathrm{~mm}$ (nominal) diameter glass rods (borosilicate, Swift Glass Co. Inc., Elmira, NY, USA) and 4.72 mm (nominal) diameter PMMA rods (type CLR, extruded Acrylic rod, Piedmont Plastics, Scarborough, ON, Canada) at a $10 \mathrm{~mm}$ nominal nozzle standoff from the initial un-machined surface of the rod. The abrasive jet footprint diameter was about $2 \mathrm{~mm}$ at that standoff. The average diameter ( \pm standard deviation of 8 measurements) over an $11 \mathrm{~cm}$ length was $4.99 \pm 0.04 \mathrm{~mm}$ for glass, and $4.72 \pm 0.01 \mathrm{~mm}$, for PMMA. Care was taken to ensure that the center of the abrasive jet remained along the rod axis as it moved in the feed direction of the stage during machining. The powder mass flow rate was $1.8 \mathrm{~g} / \mathrm{min}$. The experiments were conducted using a $760 \mu \mathrm{m}$ inner-diameter nozzle (Comco, Inc. Burbank, CA, USA) which was kept stationary at $\theta=90^{\circ}$ to the longitudinal axis of the rod.

The aim of the AJM lathe experiments was to measure the material removal rates under different process conditions and use these data to develop and test a model for the process. The glass and PMMA rods were machined at a constant rotational speed of $57 \mathrm{rpm}$ while the linear stage was moved back and forth using 8 different axial feed speeds, each over a different $9 \mathrm{~mm}$ long segment of the rod. The effective net footprint of the abrasive jet on the rod during this combined rotational and translational motion was a helix. The helix angle which determined the pitch, depended on the ratio of the rotational and feed speeds as illustrated in Fig. 5.1b. Various helix angles from $10^{\circ}$ to $80^{\circ}$ in $10^{\circ}$ increments (Table 1) were produced while the particle dose to the surface maintained constant (Fig. 5.1a). All machining experiments were conducted twice, each time without switching off the micro-blaster to minimize fluctuations in mass flow rate. The resulting reductions in the radii of the rods were measured using an optical profilometer (NANOVEA ST400 Micro Photonics Inc., Irvine, CA, USA) having a depth resolution of $25 \mathrm{~nm}$ and a lateral resolution of $0.1 \mu \mathrm{m}$. Measurements were made at three locations $2 \mathrm{~mm}$ apart on the eroded portion along the axis of each rod, and at each location the radius was measured at three angular positions approximately $120^{\circ}$ apart. 


\begin{tabular}{|c|c|c|}
\hline $\begin{array}{c}\text { Axial feed speed } \\
(\mathrm{mm} / \mathrm{s})\end{array}$ & $\begin{array}{c}\text { Helix angle } \\
\text { (degree) }\end{array}$ & $\begin{array}{c}\mathrm{N} \\
\text { (number of } \\
\text { machining passes) }\end{array}$ \\
\hline 85.07 & 10 & 255 \\
\hline 41.21 & 20 & 124 \\
\hline 25.98 & 30 & 78 \\
\hline 17.88 & 40 & 54 \\
\hline 12.59 & 50 & 38 \\
\hline 8.66 & 60 & 30 \\
\hline 5.46 & 70 & 16 \\
\hline 2.64 & 80 & 8 \\
\hline
\end{tabular}

Table 5.1 Axial feed speeds used to obtain the specified helix angles for a constant rotational speed of $57 \mathrm{rpm}$ in the AJM lathe experiments.

\subsubsection{Spring-masked helical channels on rods}

The machining of helical channels on the glass and PMMA rods was greatly simplified using steel springs as erosion-resistant contact masks (Fig. 5.1a). Without a contact mask, the depth and width of channels would be severely limited by the AJM footprint size. Even with a shadow mask between the nozzle and the rod [83] to decrease the footprint size, the machining of a helical channel without a contact mask would still require a complex motor control system to synchronize the rod rotation and the axial feed.

Steel helical tension springs were developed in the present work as a novel and simple means of creating a contact mask that could be accurately adjusted to control the pitch and width of the helical channels. The other method considered was using RapidMask (RM) which is a selfadhesive, ultra-violet light-cured polymeric mask for machining channels and making patterns. For example, Ahmadzadeh et al. [18] investigated RM for making microfluidic channels on flat targets. However, applying RM to the rod was challenging and time-consuming due to positioning difficulties and the presence of seams between the edges of the film in various wrapping configurations. 
The powder mass flow rate was kept at $2 \mathrm{~g} / \mathrm{min}$. Deep helical micro-channels on glass and PMMA were made using a nozzle scan speed of $0.2 \mathrm{~mm} / \mathrm{s}$ (in the rod axial direction, Fig. 5.1a), while for the case of shallower channels the scan speed was $1 \mathrm{~mm} / \mathrm{s}$. In both cases, the nozzle was scanned back and forth in the axial direction while the rod rotated at $500 \mathrm{rpm}$. All other process conditions were as given in Sections 2.1 and 2.3. Three spring sizes were used for the glass rods: music wire steel extension springs (W.B. Jones Spring Co., Inc., Kentucky, USA) with inner diameters of $1.94 \pm 0.02 \mathrm{~mm}$ (500 $\mu \mathrm{m}$ wire thickness), $3.00 \pm 0.05 \mathrm{~mm}$ (400 $\mu \mathrm{m}$ wire thickness), and $4.93 \pm 0.05 \mathrm{~mm}(700 \mu \mathrm{m}$ wire thickness). There was no gap between the spring coils initially at zero extensional load. A spring of inner diameter $4.70 \pm 0.02 \mathrm{~mm}$ (400 $\mu \mathrm{m}$ wire thickness) was used as the mask for PMMA rod. The two end coils of the springs were pulled beyond their elastic limit using pliers in order to obtain the desired pitch (distance between two consecutive spring coils) and were clamped to the rod by twisting the end coils against the rod and fixing them with tape. Channels with pitches of $600 \mu \mathrm{m}, 700 \mu \mathrm{m}, 750 \mu \mathrm{m}, 800 \mu \mathrm{m}$ and $900 \mu \mathrm{m}$ were machined. The springs were long enough $(250 \mathrm{~mm})$ to ensure that the pitch was uniform in the rod centre, away from the first few coils at each end.

As with the unmasked machining of rods (Section 2.3), the machining was performed at a constant rotational speed while the whole set up, Fig. 5.1a, oscillated with a constant axial feed speed back and forth along the axis of the rod. Helical channels of different depth were machined using a discrete stepped arrangement without switching off the blaster in order to reduce the effect of possible fluctuations in the mass flow rate. The glass rods were divided into 5 (low aspect ratio) and 8 (high aspect ratio) $10 \mathrm{~mm}$ long segments corresponding to 1, 3, 5, 7, 9 and 1, 3, 5, 7, 9, 11, 13, 15 nozzle passes, respectively. For the PMMA rods, the channels were machined up to 13 and 17 nozzle passes for low and high aspect ratios, respectively.

The optical profilometer was used to measure the shape and roughness of the helical channels. The cross sectional profiles of the masked channels were measured at three angular positions approximately $120^{\circ}$ apart for each nozzle pass. To ensure that the rod was also aligned with the profilometer scan direction, cross-sectional scans were made across 4 different un-eroded sections, and the rod was re-aligned manually until 4 such profiles were coincident to within $0.01 \%$. A bracket was then attached to the profilometer stage to preserve the alignment for subsequent measurements. 


\subsection{Modelling}

\subsubsection{Prediction of surface evolution for masked helical channels}

Ghobeity et al. [9] presented a surface evolution model that could predict the crosssectional profiles of channels machined in initially flat brittle materials using AJM. Considering only the normal component of particle velocity, the model was given as:

$$
z_{,}-E(x)\left(1+z_{, x}\right)^{-k / 2}=0
$$

where $z_{t}$ and $z, x$ are the partial derivatives of the profile depth $z$ with respect to time and the coordinate $x$, defining the width dimension, $E(x)$ is the erosive efficacy distribution seen by the exposed target surface in a single pass of the nozzle (i.e. the lateral spatial distribution of the potential of the jet to erode), and $k$ is a velocity exponent related to the erosive characteristics of the surface material. Therefore, $E(x)$ accounts for the erosion generated by the entire jet footprint as it passes a given point.

The erosion of ductile materials has a more complex dependence on the local impact angle. Thus, the modified surface evolution model is given in [6] as:

$$
z_{t}-E(x) \sqrt{\left(1+z,{ }_{x}^{2}\right)} g(\alpha)=0
$$

where

$$
\mathrm{g}(\alpha)=(\sin \alpha)^{n_{1}}\left(1+H_{v}(1-\sin \alpha)\right)^{n_{2}}
$$

which describes the dependence of the erosion rate on the impact angle, $\alpha$, defined between the incident velocity vector and the local tangent to the surface as:

$$
\alpha=\frac{\pi}{2}-\arccos \left(\frac{1}{\sqrt{1+z, x^{2}}}\right)
$$

$H_{v}(\mathrm{GPa})$ is the initial target hardness and the constants $n_{1}$ and $n_{2}$ are determined experimentally and depend on the particle hardness and other impact conditions [12]. Equations (5.1) and (5.2) were valid for the cases that the neglect of jet divergence and variations in the local standoff distance were insignificant. 
Nouhi et al. [66] proposed a modification to the above models considering the variation in both the local nozzle standoff distance and the divergence angle of each particle trajectory in the jet plume which enabled the modified model to predict the channel profiles machined on curved and tilted surfaces. The modified surface evolution models for arbitrarily-shaped brittle and ductile materials presented by Nouhi et al. [66] were:

$$
\begin{gathered}
z,_{t}=E(x, z)\left(1+z,{ }_{x}{ }^{2}\right)^{-k / 2}\left(\frac{z-x z,_{x}}{\sqrt{x^{2}+z^{2}}}\right)^{k+1} \\
z,_{t}=E(x, z) \sqrt{1+z,_{x}^{2}} g\left(\frac{\pi}{2}-\cos ^{-1}\left(\frac{z-x z,_{x}}{\sqrt{x^{2}+z^{2}} \sqrt{1+z,_{x}^{2}}}\right)\right)
\end{gathered}
$$

where the $x$ (width direction) and $z$ (depth direction) coordinates are attached to the nozzle. For unmasked machining using nozzles typical of AJM operations, $E(x, z)$ can be expressed as:

$$
E(x, z)=\delta\left(\frac{\beta}{z}\right)^{2} e^{-\beta^{2} \frac{x^{2}}{z^{2}}}
$$

with

$$
\delta=\frac{C}{\rho_{s}} \frac{\dot{M}}{\pi}|V|^{k}
$$

The $C$ and $k$ are constants related to the erosive characteristics of the surface material of density $\rho_{s}, \dot{M}$ is the mass flow rate, $V$ is the particle velocity, $\beta$ is the nozzle "focus coefficient", with higher values indicating a more focused stream, and $\delta(\beta / z)^{2}$ is proportional to the channel depth for a given particle dose. As with Eq. (1), $E(x, z)$ can be used to characterize the erosive efficacy to either a stationary surface, such as in the machining of holes, or one that moves, such as in the machining of channels generated by the entire jet footprint as it passes a given point. For a cylindrical target (Fig. 1), when the model is implemented to predict channel cross-sectional profiles in the $x-z$ plane, the initial condition is $z(x, 0)=h$, while when channel cross-sectional profiles are to be predicted in the $z-y$ plane, it is $z(y, 0)=(h+R)-\sqrt{R^{2}-y^{2}}$, where $h$ is the nozzle standoff distance and $R$ is the cylinder radius. 
In the present work, the model of [66], Eqs. (5.5) and (5.6), which take into account the change in local nozzle standoff distance and the local impact angle of particles, was implemented to predict the evolving shape of masked helical channel profiles machined on glass and PMMA rods (discussed in Section 5.4.2.1). As discussed by Getu et al. [6] for masked AJM, the erosive efficacy is limited in width, and therefore any suitable curve fit of a shallow eroded profile can be used to obtain $E(x, z)$. Therefore, for the masked helical channels, instead of Eq. (5.7), a cubic spline curve fit of a measured shallow masked channel profile was used to obtain $E$. As discussed by Ghobeity et al. [51], if the profile used to obtain $E$ is shallow, the profile itself and $E$ have the same functional form. This shallow channel was representative of the combined effects of: (i) the sum of the erosive efficacy over the jet footprint as it moved over the rod during the rotation, (ii) the contributions to the erosive efficacy of both the incoming particle flux and particle ricochets off the mask edges, (iii) the particle velocity distribution, and (iv) the divergence of particle trajectories across the incident jet that gave rise to the primary first-strike footprint [51].

\subsubsection{Prediction of volume of removed material in unmasked AJM lathe operations}

Lathe operations to reduce the rod radius result in an instantaneous noncircular crosssection only at the leading edge of the machining front. Therefore, provided that multiple rapid lathe passes are used such that the slope of the machining front remains small, the rod can be assumed to be everywhere and at any time uniformly circular. However, as the jet footprint moves along the helical path, the curvature implies a nonzero slope $z, y$, which is independent of $x$. Therefore, Eqs. (5.5) and (5.6) can be generalized to the three-dimensional case, for brittle and ductile erosive systems, respectively as:

$$
\begin{gathered}
z_{t}=\delta\left(\frac{\beta}{z}\right)^{2} e^{-\beta^{2}\left(\frac{x^{2}+y^{2}}{z^{2}}\right)}\left(1+z,{ }_{x}{ }^{2}+z,{ }_{y}{ }^{2}\right)^{-k / 2}\left(\frac{z-y z, y-x z, x}{\sqrt{x^{2}+y^{2}+z^{2}}}\right)^{k+1} \\
z,_{t}=\delta\left(\frac{\beta}{z}\right)^{2} e^{-\beta^{2}\left(\frac{x^{2}+y^{2}}{z^{2}}\right)} \sqrt{1+z,{ }_{x}{ }^{2}+z,,_{y}{ }^{2}} g\left(\frac{\pi}{2}-\cos ^{-1}\left(\frac{z-y z, y-x z, x}{\sqrt{x^{2}+y^{2}+z^{2}} \sqrt{1+z, x^{2}+z, y^{2}}}\right)\right)
\end{gathered}
$$


where the origins of $x, y, z$ are shown in Fig. 5.1. These expressions account for the variation of local standoff distance and the angular dependency of erosion brought about by the curvature of the target at any instant during the passage of the jet footprint. In the case of unmasked lathe machining, Eqns. (5.9) and (5.10) can be integrated with respect to time over the helical path a footprint moves as the rod rotates and translates to obtain the profile resulting from a single rotation.

The pitch of the helical path described by the jet footprint as it moves axially on the rotating rod, $c$, and the scanning period of each machining cycle, $T,(90 \mathrm{~mm}$ axially along the rod, back or forth) could be calculated knowing the helix angle as determined by the axial feed speed and the rotation speed. The parametric equation of the cylindrical helix followed by the jet footprint is given by:

$$
\vec{R}(\theta)=c \vec{i}+r \sin \theta \vec{j}+r \cos \theta \vec{k}
$$

where $r$ is the radius of the cylindrical helix and $\theta$ is the angular coordinate in Fig. 5.2. As indicated in Fig. 5.1b, the helix angle is defined by the rotational speed, $r \omega$, and the axial feed speed, $V_{f}$,

$$
\psi=\tan ^{-1}\left(\frac{r \omega}{V_{f}}\right)
$$

$\Psi$ is also given by

$$
\cos \psi=\frac{\vec{j} \cdot \overrightarrow{R^{\prime}}(\theta)}{|\vec{j}|\left|\overrightarrow{R^{\prime}}(\theta)\right|}=\frac{c}{\sqrt{r^{2}+c^{2}}}
$$

where $c$ is the pitch of the spring divided by $2 \pi$ (Fig. 5.2), $\vec{j}$ is the unit vector in $y$ direction, $\overrightarrow{R^{\prime}}(\theta)$ is the derivative of the position vector with respect to $\theta$, Eq. (5.11); i.e. tangent to $\vec{R}(\theta)$. Combining Eqs. (5.12) and (5.13) yields the following expression for $c$ :

$$
c=\frac{r \cos \psi}{\sqrt{1-\cos ^{2} \psi}}
$$

Since $c \theta=c \omega t$, the machining time, $T$, required to scan a length $L$ of the rod is 


$$
T=\frac{L}{c \omega}
$$

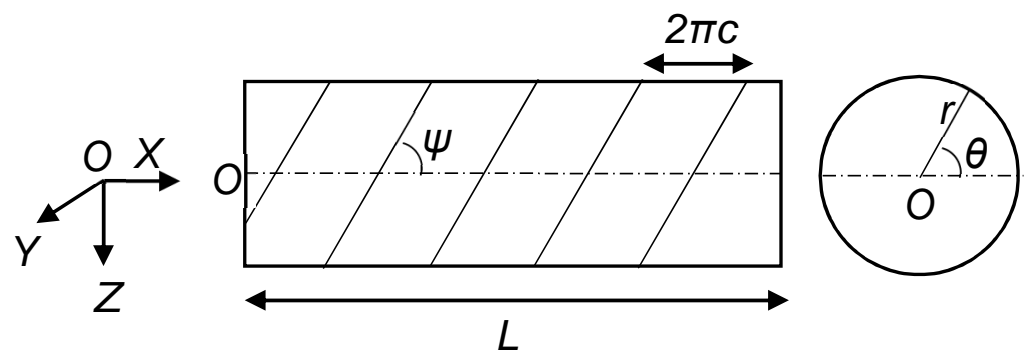

Fig. 5.2 Definition of helix pitch, $c$, helix angle, $\psi$, radius at the rod surface, $r$, and angular position, $\theta$. The coordinate system is shown with an offset from its actual origin, point $O$, for clarity.

The origin of the coordinate system in Eqs. (5.11) to (5.15) was taken to be the left end of the rod, point $O$ in Fig. 5.2, while it was located at the nozzle elsewhere in the paper.

1. The erosive efficacy of the jet was first obtained by a curve fit of Eq. (7) to a shallow hole machined in a flat target with the nozzle stationary. As discussed in Ref. [66], the fit is independent of standoff distance for conically spreading jets. In order to take into account the changes in angle of attack and standoff brought about by the rod curvature, Eqs. (5.9) and (5.10) must be integrated with respect to time during the helical passage of the footprint over the rod (Fig. 5.3) in a single rotation. If it assumed that the material removed during a single rotation is small, then $z_{x}=0$ and the time integrals of Eqs. (5.9) and (5.10) yield:

$$
Z=\int_{0}^{t^{*}} \delta\left(\frac{\beta}{z}\right)^{2} e^{-\beta^{2}\left(\frac{x^{2}+y^{2}}{z^{2}}\right)}\left(1+z,{ }_{y}{ }^{2}\right)^{-k / 2}\left(\frac{z-y z, y}{\sqrt{x^{2}+y^{2}+z^{2}}}\right)^{k+1} d t
$$


for the glass (brittle) rods, and

$$
Z=\int_{0}^{t^{*}} \delta\left(\frac{\beta}{z}\right)^{2} e^{-\beta^{2}\left(\frac{x^{2}+y^{2}}{z^{2}}\right)} \sqrt{1+z,_{y}^{2}} g\left(\frac{\pi}{2}-\cos ^{-1}\left(\frac{z-y z, y}{\sqrt{x^{2}+y^{2}+z^{2}} \sqrt{1+z, y^{2}}}\right)\right) d t
$$

for the PMMA (ductile) rods. $Z$ is the profile resulting from one rotation of the rod over the jet footprint, and $x, y$ and $z$ vary with time as the footprint follows a helical path over the rod surface such that

$$
\begin{gathered}
x(t)=v_{f} t \\
y(t)=r_{f}+R(1-\cos (\omega t)) \\
z(t)=(R+h)-\sqrt{R^{2}-y(t)^{2}} \\
z_{y}(t)=\frac{y(t)}{\sqrt{R^{2}-y(t)^{2}}}
\end{gathered}
$$

where $t^{*}=2 S_{f} / v_{r}$ is the time taken for a given axial line on the rod surface to rotate under the jet footprint. (Fig. 5.3). $R$ is the radius of the rod, $h$ is the nozzle standoff distance, $S_{f}=R \alpha$ is half of the maximum arc length of the footprint on the rod surface (Fig. 5.3), $v_{r}$ and $v_{f}$ are the rotational and axial feed speeds, respectively, and $r_{f}$ is the horizontal projection of $S_{f}$ in the direction of the $y$ axis (Fig. 5.3). The angle $\alpha$ defining the footprint (Fig. 5.3) was determined by making measurements on one jet footprint on the rod using the profilometer. The profile of Fig. 5.4 is an example of the result of integrating Eq. (5.16) in this manner. It is noted that although the path of the footprint was along a helix, the profile nevertheless appears to be symmetric in $x-z$ plane because it is so shallow. It is also noted that the slope $z, x$ is indeed small, as was assumed earlier.

2. The translational motion of the rotating rod resulted in a helical machining path described by Eq. (5.11) over a length $L$ (Fig. 5.2). Its cross-sectional profile is everywhere described by $Z$ obtained in step 1. As the rotating rod translated in the reverse direction under the stationary jet, a new helical channel having the identical $Z$ profile was machined on the rod, offset by a distance 
$d$ (Fig. 5.5), which was known from the recorded instantaneous $x$-position of the stage. The two offset channel profiles were superimposed at their points of intersection, obtained by equating the helix equations, taking into account the channel width. If the helical footprints overlapped in a single translational pass (depending on the $Z$ profile width and helix pitch $2 \pi c$ ), then they were superimposed each rotation, otherwise, they were superimposed each translational pass; i.e. after each time $T$ passed (Eq. (5.15)). The superposition process was continued to the end of the machining time, yielding the axial cross-sectional profile of the eroded surface on the rod (Fig. 5.5). Finally, the total volume of the removed material was calculated using Pappus's theorem; i.e. the $2 \mathrm{D}$ area bounded between the obtained profile and the uneroded surface of the rod was rotated by $2 \pi$.

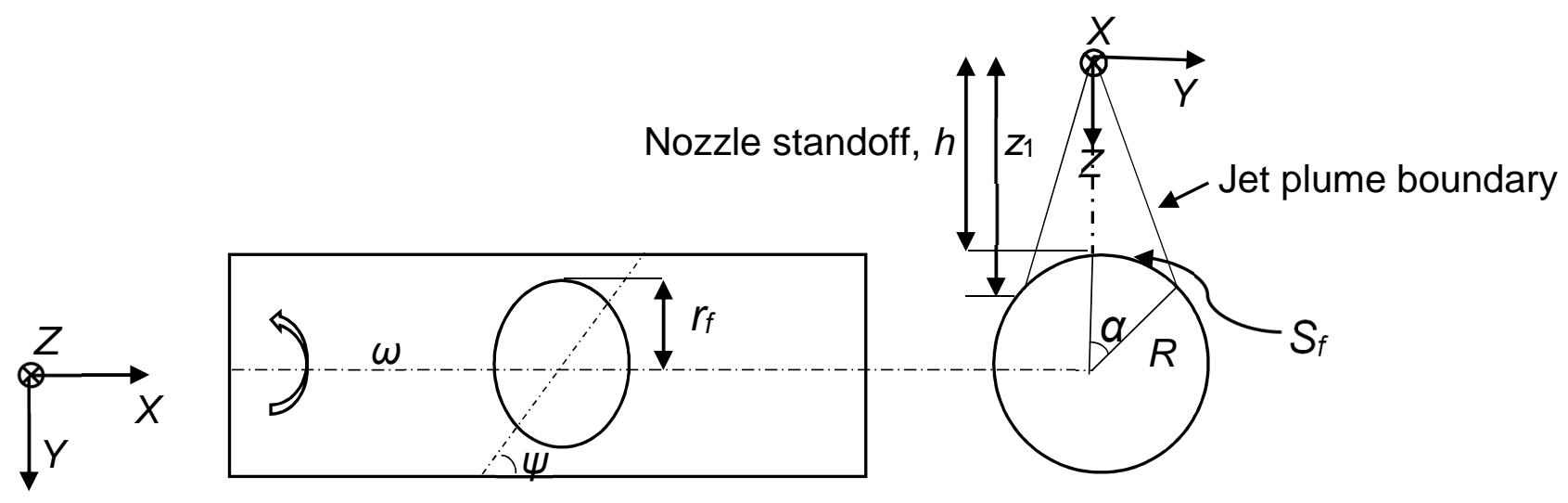

Fig. 5.3 A schematic (top view) of the projection of the circular jet on the rod giving an elliptical footprint (the helix angle, $\psi$ shows the direction of footprint movement) (left). Side view of the rod (right) representing the range of $z$ on one footprint, $h$ to $z_{1}$. Coordinate system attached to the nozzle. 


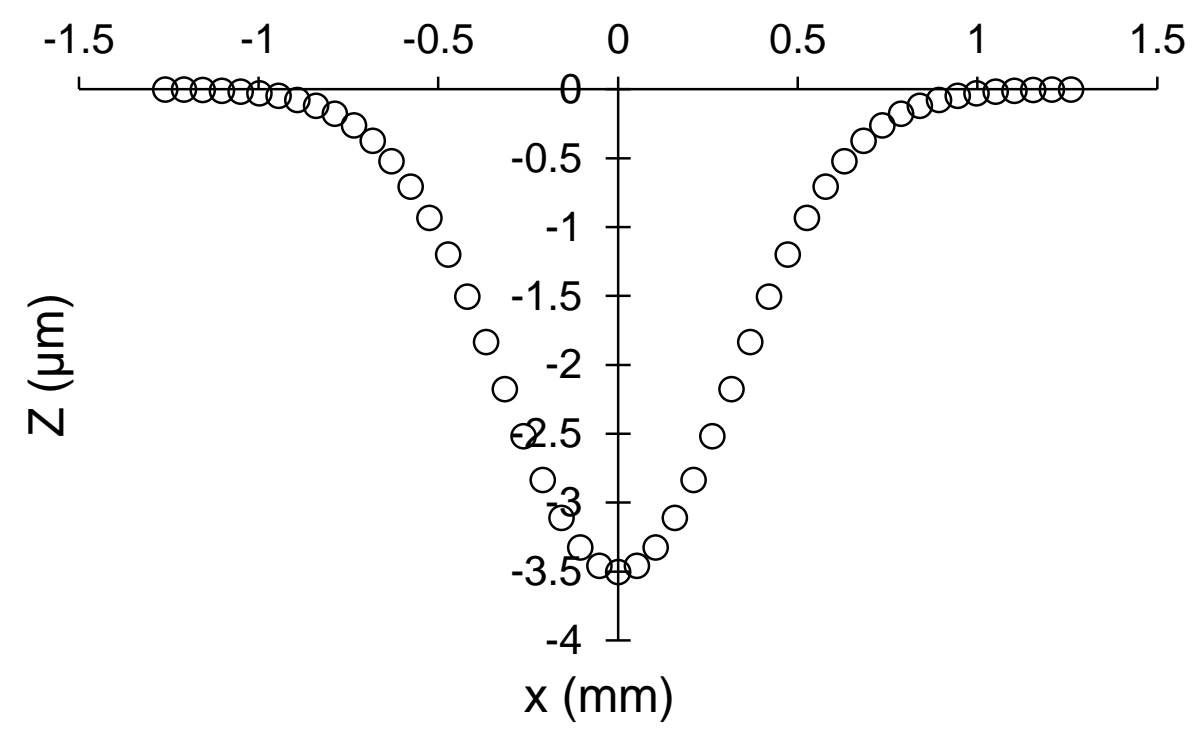

Fig. 5.4 Example of rod profile on a $5 \mathrm{~mm}$ diameter glass rod resulting from rotation over one footprint at a $10 \mathrm{~mm}$ standoff distance (helix angle 10 $0^{\circ}$ (according to coordinates of Fig. 5.3).

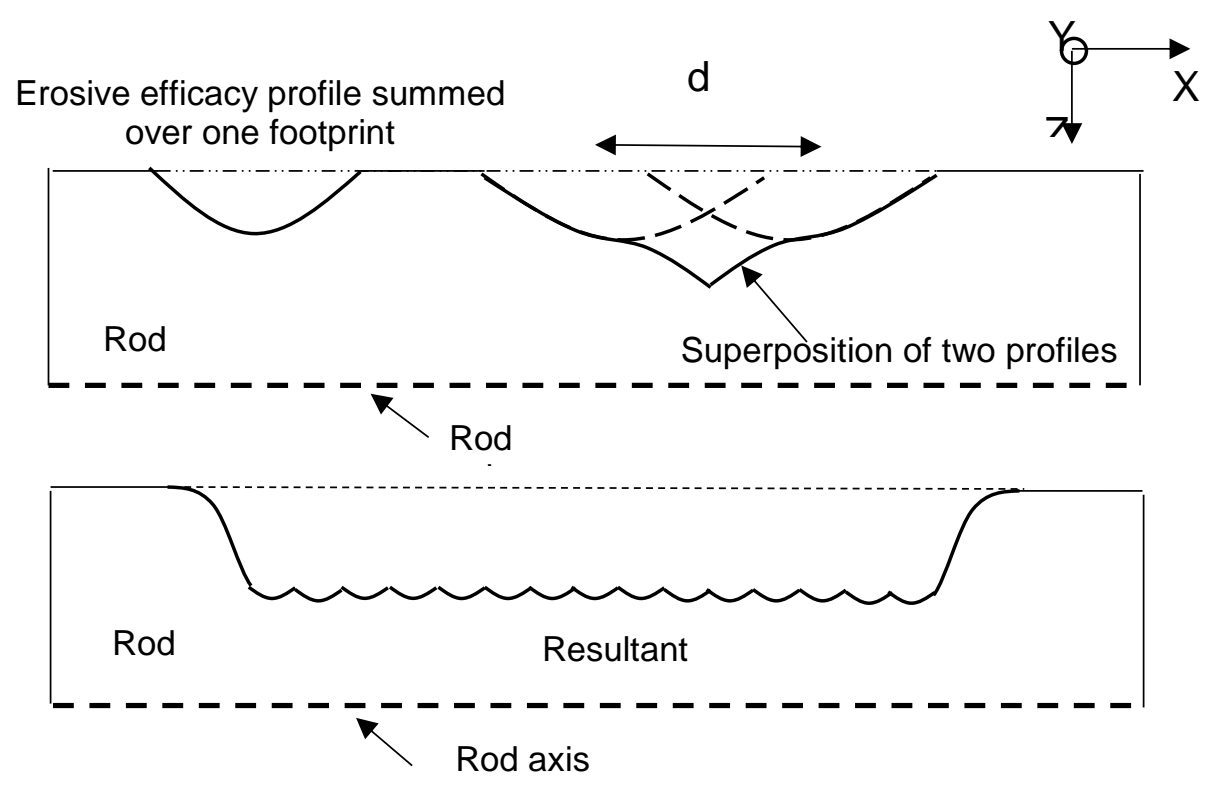

Fig. 5.5 Schematic of the process of superposition to obtain the resultant profile for unmasked lathe machining of rods. Cross-sectional view of half the rod. 


\subsection{Results and discussion}

\subsubsection{Unmasked AJM lathe operations}

\subsubsection{Volume of removed material}

Figures 5.6a and 5.6b show that the measured volumetric machining rates for the turning of glass and PMMA rods, closely matched the predictions of Section 3.2 (Eqs. (5.9) and (5.10), respectively). The parameters $\delta$, Eq. (8), and the nozzle focus coefficient $\beta$ were obtained by fitting Eq. (5.7) to the measured cross-sectional profiles of shallow holes machined in a flat glass and PMMA surface, giving $\delta=0.0007 \mathrm{~mm}^{3} / \mathrm{s}$ for glass, $\delta=0.0004 \mathrm{~mm}^{3} / \mathrm{s}$ for PMMA, and $\beta=23$. The predicted values were on average $9 \%$ greater for the $5 \mathrm{~mm}$ glass rod and $4 \%$ greater for the 4.72 mm PMMA rod. The maximum measured slope of the jet impact area in the scan direction (the leading edge of the machining front) was just $2^{\circ}$ at a helix angle of $10^{\circ}$; therefore, the leading edge slope could not contribute to these small over-predictions. It is hypothesized that the errors are due to two assumptions of the model, and that the errors may compete since they sometimes may have opposite effects. Firstly, there was an error associated with the assumption of $z, x=0$. Especially at high helix angles, when a low axial feed is used, the channels are deeper and therefore can develop more significant slopes. Because the erosion for brittle systems is maximum at perpendicular incidence, whereas for ductile systems (Eq. (5.3)) it is maximum at a shallow angle, the effect of this error is different for the two systems. The model assumes the slope zero, which for glass targets implies a predicted erosion which is higher than actual, while for PMMA targets, it implies a predicted erosion which is lower than actual. The second error arises from the fact that the rod radius $R$, is assumed constant at its uneroded value in Eqs. (5.18-5.21). In reality, $R$ will decrease as erosion progresses, and thus $\alpha$ (Fig. 5.3) will initially increase, leading to a wider than assumed range of impact angles at which the particle dose is delivered. The narrower assumed range of impact angles will tend to overestimate the erosion for glass, and underestimate it for PMMA. Should $R$ become so small that the particles at the periphery of the conically spreading jet no longer strike the rod, then a lower than assumed dose of particles is actually delivered and, regardless of material, the model would overpredict the erosion.

Figure 5.6c shows two axial cross-sectional profiles (helix angles of $10^{\circ}$ and $80^{\circ}$ ) machined over a length of $9 \mathrm{~mm}$ on a $5 \mathrm{~mm}$ glass rod, and the corresponding model predictions. As expected, 
the waviness increased as the helix angle decreased simply because there was less overlap of the adjoining helical channels. For example, the waviness at $80^{\circ}$ was $2.5 \mu \mathrm{m}$, while it was $13 \mu \mathrm{m}$ at $10^{\circ}$. By further increasing the helix angle, smoother surfaces can be obtained.

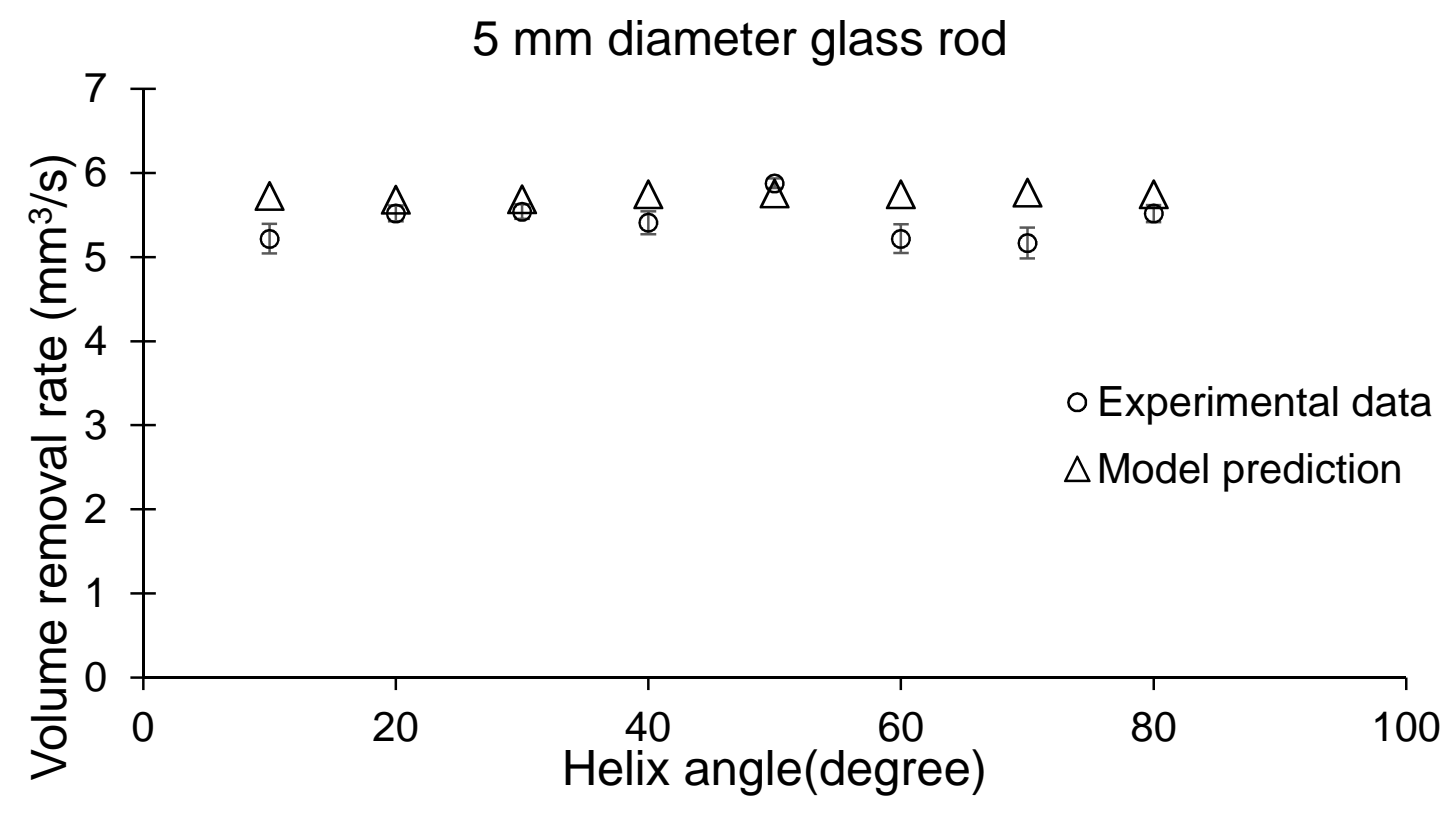

(a)

4.72 mm PMMA rod

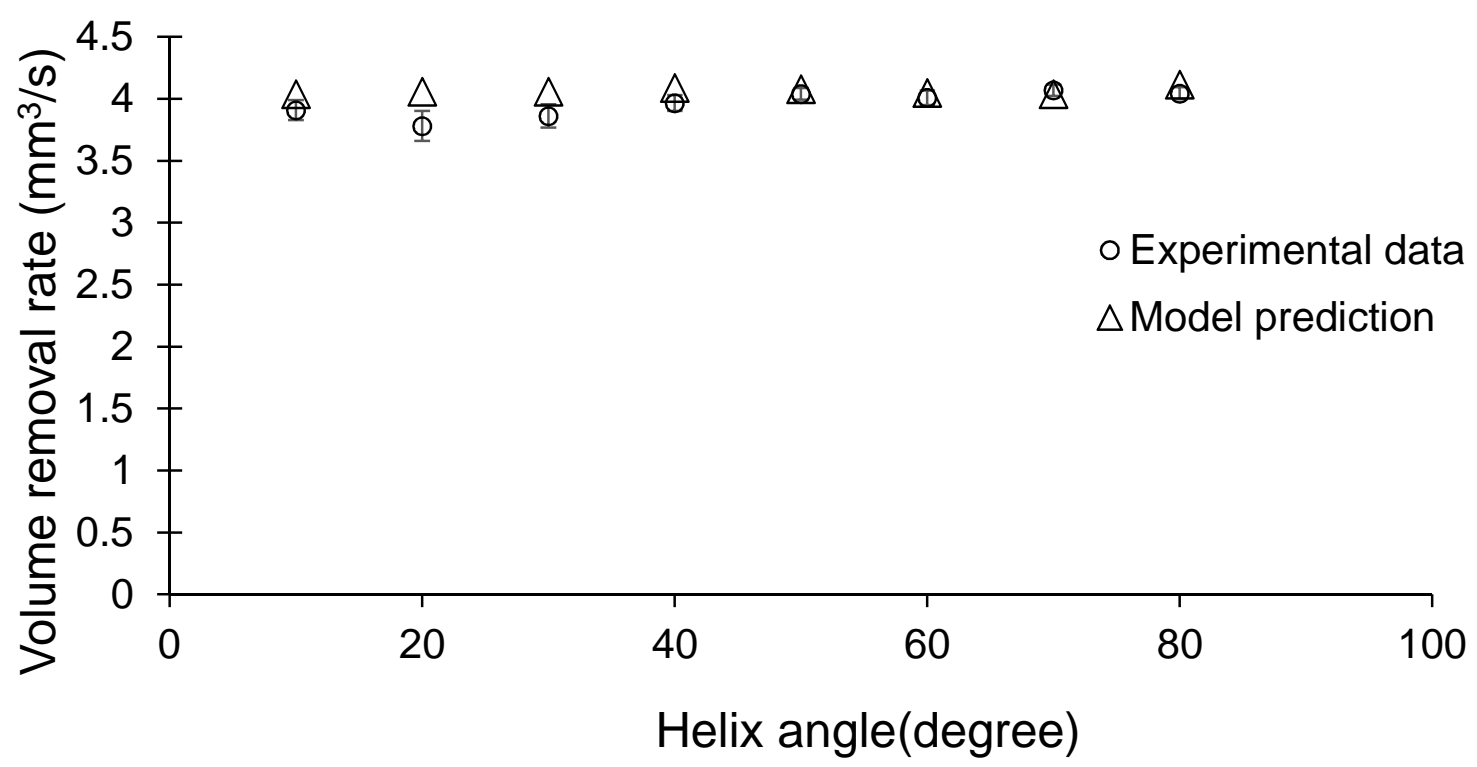

(b) 


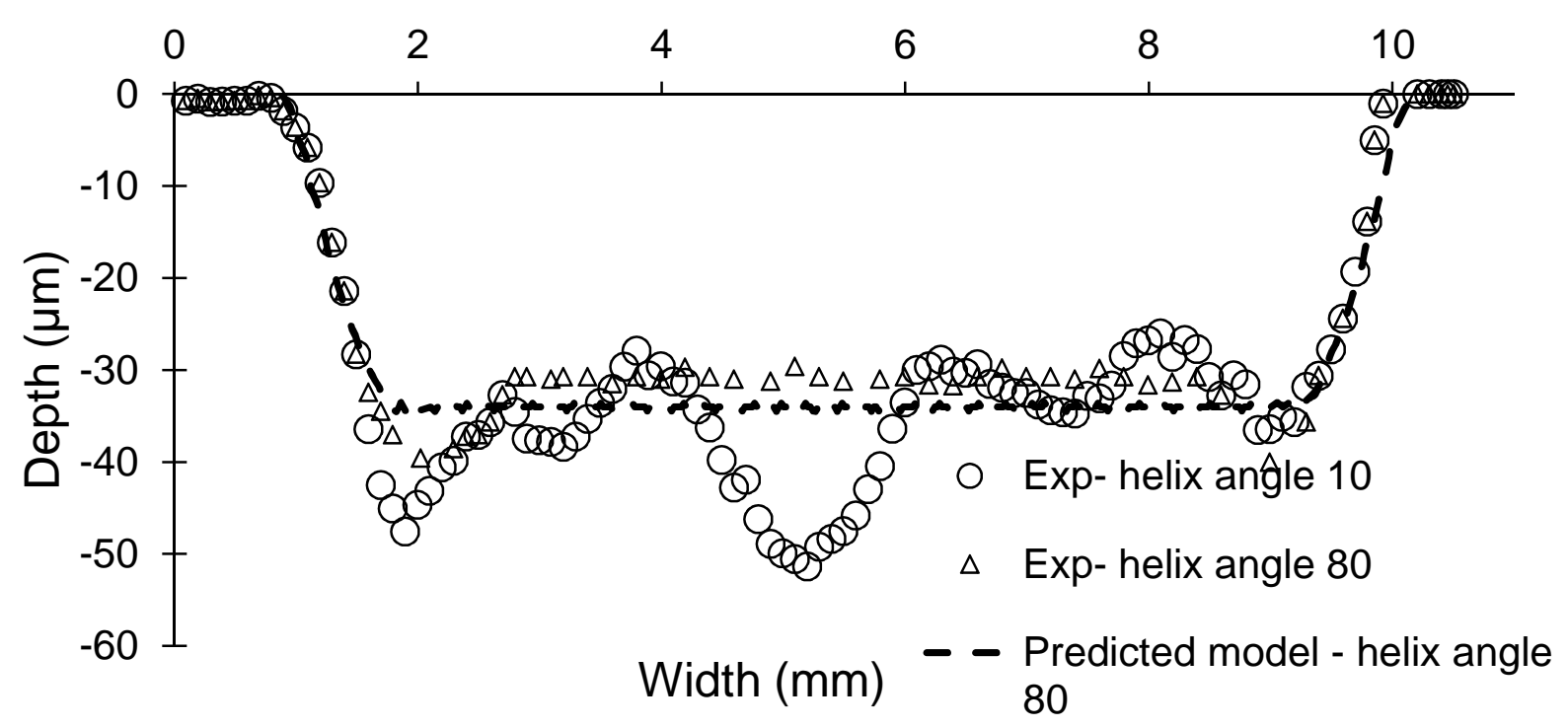

(c)

Fig. 5.6 Measured and predicted AJM lathe machining rate for: a) $5 \mathrm{~mm}$ glass rod, b) $4.72 \mathrm{~mm}$ PMMA rod. Predictions used the model of Section 5.3.2, c) measured cross sectional profile and model prediction for $5 \mathrm{~mm}$ diameter glass rod. The experiments were repeated twice and measurements were performed three times (i.e., the data points represent the average of 6 measurements).

\subsubsection{Effect of nozzle offset}

Since the erosion rate of PMMA reaches a maximum when the impact angle is approximately $25^{\circ}$, and is at least $80 \%$ of this maximum over the range $10^{\circ}-45^{\circ}$ [6], higher machining rates could be achieved by offsetting the nozzle centerline from the rod axis (Fig. 5.77) so that the particles struck the rod at smaller average angles. An experiment was conducted to measure the machining rate of a $4.72 \mathrm{~mm}$ PMMA rod rotating at $500 \mathrm{rpm}$ with a nozzle translating axially back and forth at $0.2 \mathrm{~mm} / \mathrm{s}$ at a $1.4 \mathrm{~mm}$ offset. This was the largest value for which the entire jet footprint remained on the initial rod diameter. The average impact angle for zero offset was $90^{\circ},\left(\Upsilon_{1}=\Upsilon_{2}=73^{\circ}\right.$; i.e. the range of impact angles was $73^{\circ}$ to $\left.90^{\circ}\right)$, whereas in the case of the $1.4 \mathrm{~mm}$ offset the average was $72^{\circ}\left(Y_{1}=83^{\circ}\right.$ and $Y_{2}=16.5^{\circ}$; i.e. the range of impact angles was $16.5^{\circ}$ to $83^{\circ}$ ) (Fig. 5.77). With the other experimental conditions as in Section 2.3, it was observed 
that the erosion rate increased by $15 \%$. The roughness, $R_{a}$, of the rod surface, measured in axial direction, did not change significantly with an offset nozzle.

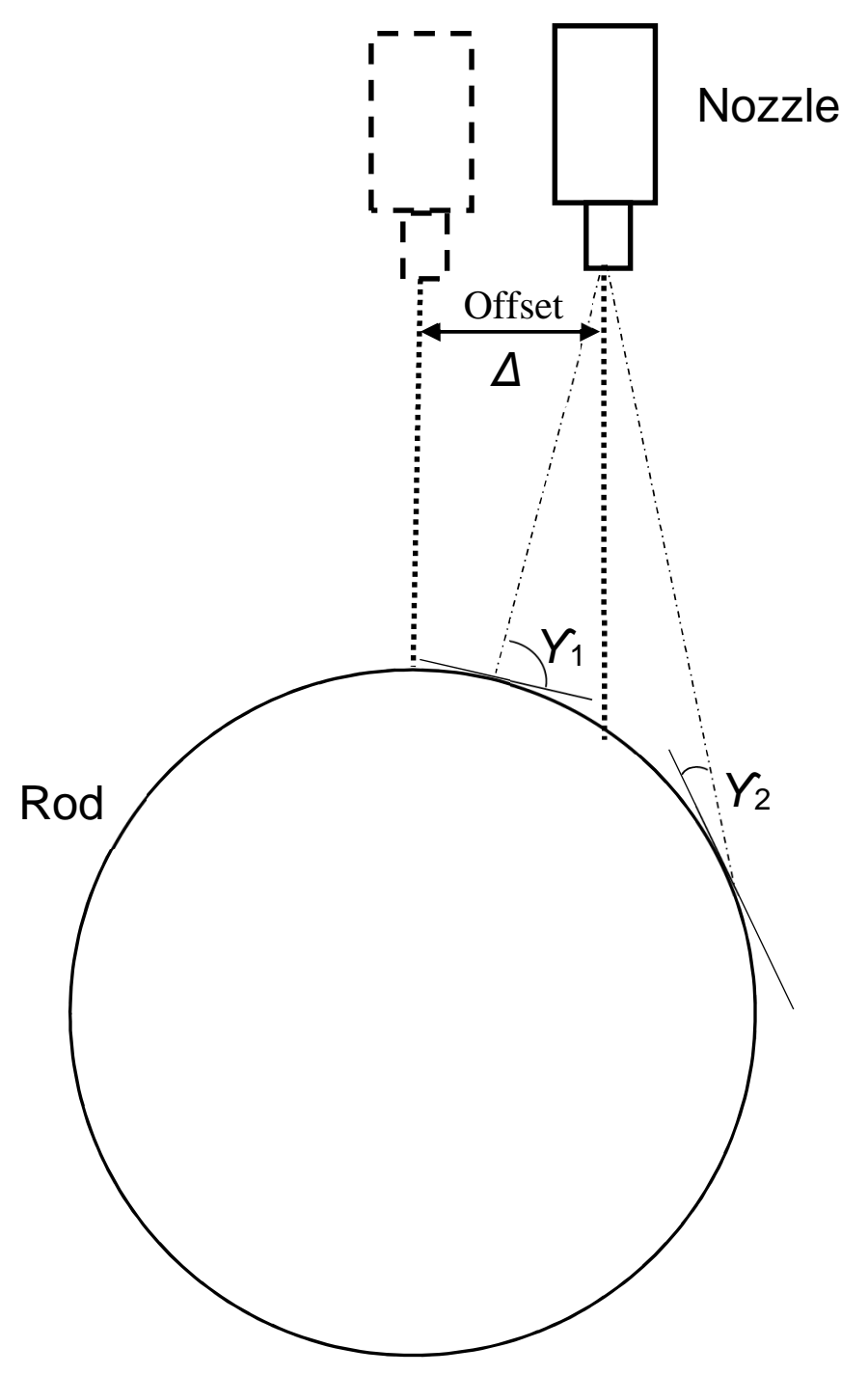

Fig. 5.7 Schematic of nozzle offset $\Delta$, with $\Upsilon_{1}$ and $\Upsilon_{2}$ the particle impact angles to the surface of the rod at the boundaries of the jet plume. 


\subsubsection{Masked helical channels}

\subsubsection{Surface evolution of helical channel profiles}

As described in Section 5.3.1, a shallow first-pass profile of the eroded masked channel was used to characterize the erosive efficacy. Therefore, the erosive efficacy already included the effect of the footprint traversing as the rod rotated, and any effects associated with the curvature and slope in the $y$ direction. It also included the effects of particle ricochet from the sides of the spring mask. In contrast to the unmasked channels of Section 5.4.1, nonzero $z, x$ slopes developed rapidly in the narrow spring-masked channels so the surface did not remain approximately uniformly circular. Therefore, the resulting profiles were predicted by solving the two-dimensional surface evolution partial differential equations, Eqs. (5.5) and (5.6), which are the same as Eqs. (5.9) and (5.10), but with the $y$-dependency removed $(y=z, y=0)$, since the erosive efficacy already included the effect of the footprint traversing in the $y$ direction.

Figures 5.9a-d show the measured cross-sectional ( $x-z$ plane) profiles of low and high aspect ratio stepped helical micro-channels machined on a $5 \mathrm{~mm}$ diameter glass rod and a $4.72 \mathrm{~mm}$ diameter PMMA rod along with the predictions of the surface evolution equations. The equations were solved numerically using the method of lines in Mathcad 15 (PTC Inc. Needham, MA, USA). The number of spatial $(\mu \mathrm{m})$ and time (s) discretization points used were 200 and 300 respectively, which ensured the accuracy and convergence of the numerical solution. The initial condition was: $z(x, 0)=h$, and the boundary conditions were $z(w / 2, t)=0$ and $z(-w / 2, t)=0$ (Fig. 5.8). 


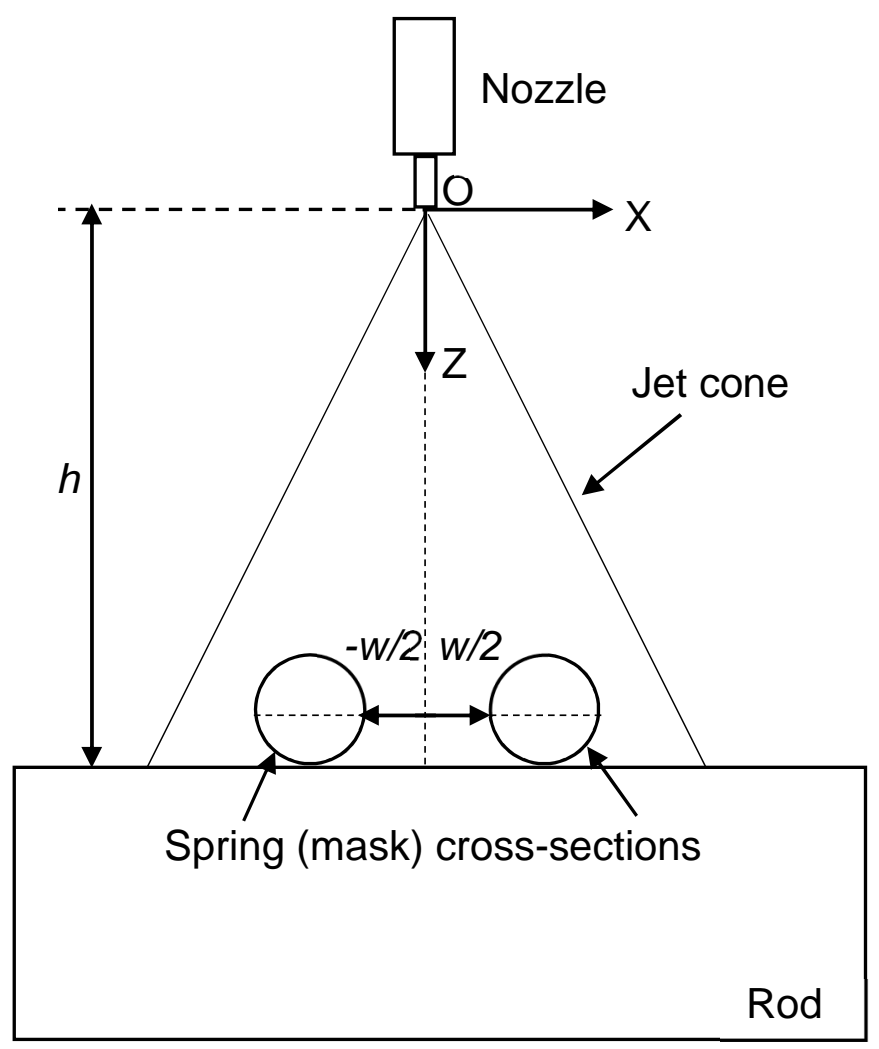

Fig. 5.8 Schematic of the location of spring mask on the rod; $w$ is the width of mask opening, $h$ is the nozzle standoff distance.

It was observed that the model could accurately predict the profile shape and there was a close agreement between the predictions and the measurements of the centerline depth. For example, the predicted centerline depth after 7 passes was $5 \%$ greater than the measured value for the glass rod (Fig. 5.9a), and for the PMMA rod the maximum error was 7\% after 9 passes (Fig. $5.9 \mathrm{c})$.

This method of machining offers a convenient way to fabricate a wide variety of helical micro-channels with different widths, aspect ratios and radii of curvature. For instance, Figs. 5.9a and $5.9 \mathrm{~b}$ show the profiles of two glass helical micro-channels which have aspect ratios up to 0.2 and 0.7, respectively, while Figs. 5.9c and 5.9d show PMMA micro-channels with aspect ratios of 0.09 and 0.4 , respectively. 
Figures 5.10a to 5.10d show optical micrographs of helical channels fabricated on a 2, 3 and $5 \mathrm{~mm}$ diameter glass rods and a $4.72 \mathrm{~mm}$ diameter PMMA rod using the procedures of Section 2.4. It is seen that the channel cross-sectional profile in the glass had a characteristic V shape, whereas that in PMMA had U shape; i.e. the PMMA channels had steeper sidewalls and flatter bottom sections than did the glass channels. This trend is well known in the AJM literature for flat surfaces and was due to the difference between brittle glass and ductile PMMA in the dependence of the erosion on the local impact angle [6]. Such differences in channel shape may offer the possibility of modifying the microfluidic flow field to suit particular applications in mixing or particle separation. Note that the marks on some of the threads in Figs. 5.10b and 5.10c are not cracks; however, care was required to avoid cracking when removing the springs from relatively deep helical micro-channels.

The average roughnesses, $R_{a}$, of the channel bottoms of the $5 \mathrm{~mm}$ glass rod and $4.72 \mathrm{~mm}$ PMMA rod were measured using the optical profilometer with a cutoff wavelength of $250 \mu \mathrm{m}$, as was suggested in [84]. The roughness of the glass rod increased with the number of passes of the nozzle; i.e. $R_{a}=0.5,0.65,1.10,2.12 \mu \mathrm{m}$ after $3,7,11$ and 15 passes of the nozzle (Fig. 5.10b) whereas the opposite trend was found in PMMA, i.e. $R_{a}=0.75,0.70,0.59,0.105 \mu \mathrm{m}$ after $3,7,11$ and 15 passes (Fig. 5.10d). These trends could be attributed to the increase in the impact angle of the particles impacting the surface of the helical channel as the radius of the rod decreased during the machining, and to the differences in erosion mechanisms for the two materials. As the channels became deeper, the rod diameter decreased until a portion of the abrasive jet plume at the periphery missed the rod entirely and did not contribute to the erosion; e.g. after 15 passes the diameter of the rod was reduced by almost $1 \mathrm{~mm}$. Another consequence of this reduction in rod diameter was a decrease in the range of impact angles as they became closer to $90^{\circ}$. For example, after 15 passes of machining the range of impact angles was $78^{\circ}$ to $90^{\circ}$ whereas at the beginning of the machining the range was $73^{\circ}$ to $90^{\circ}$. The roughness in brittle (glass) and ductile (PMMA) materials increases [85], and decreases [5], respectively ,as the angle of attack becomes more perpendicular, thus explaining the different roughness trends in the two materials as the number of passes increased. 


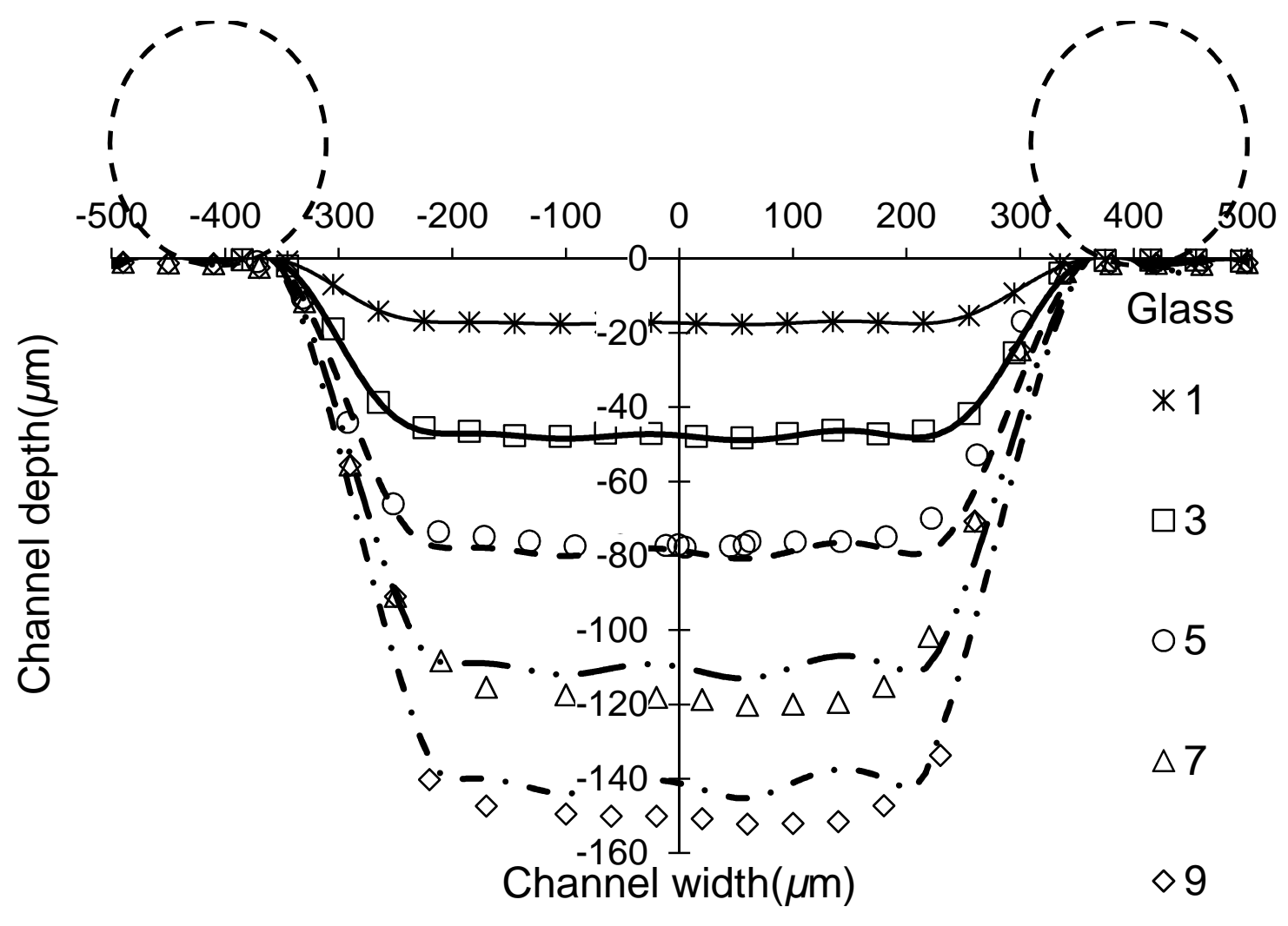

(a) 


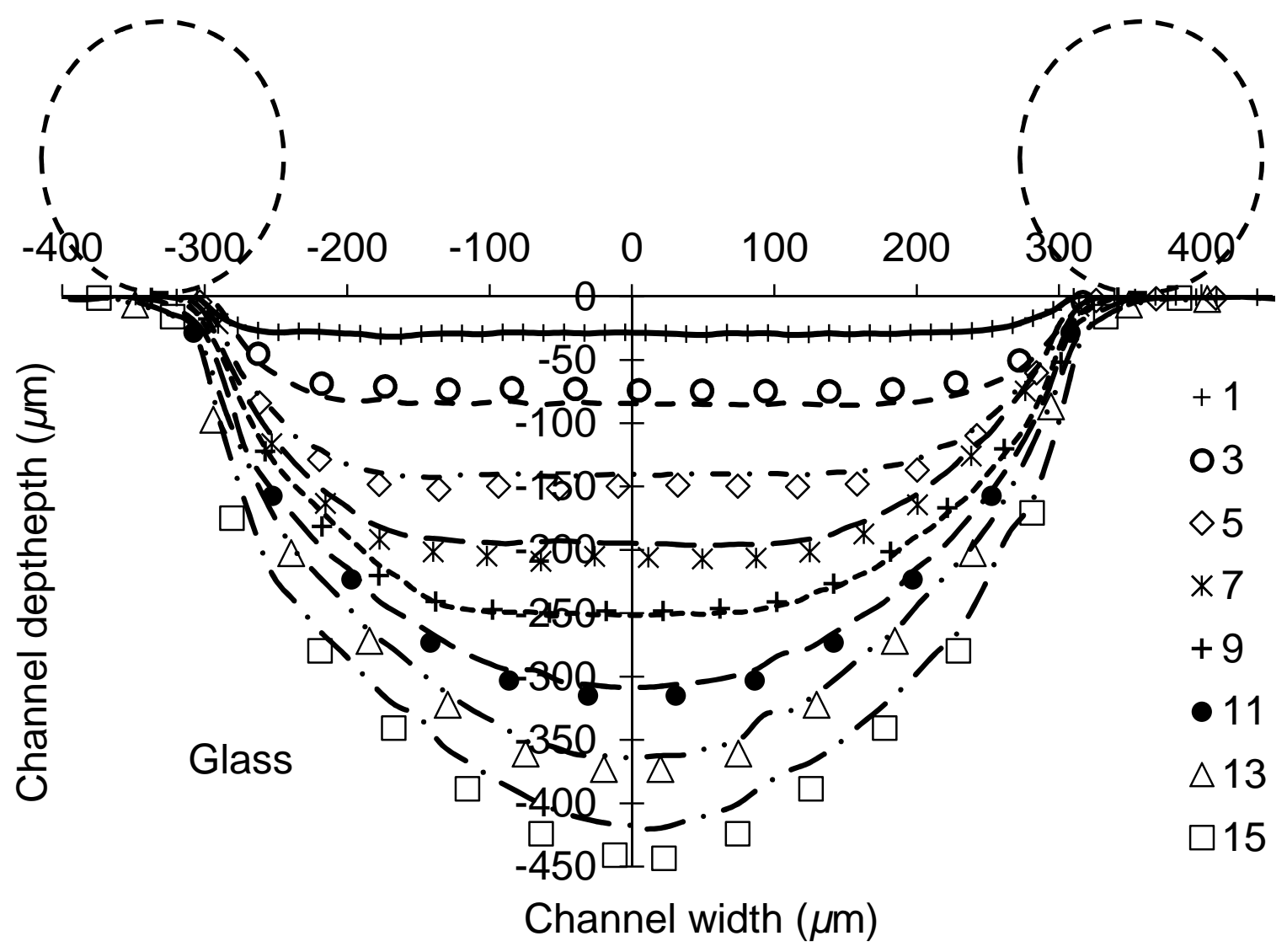

(b) 


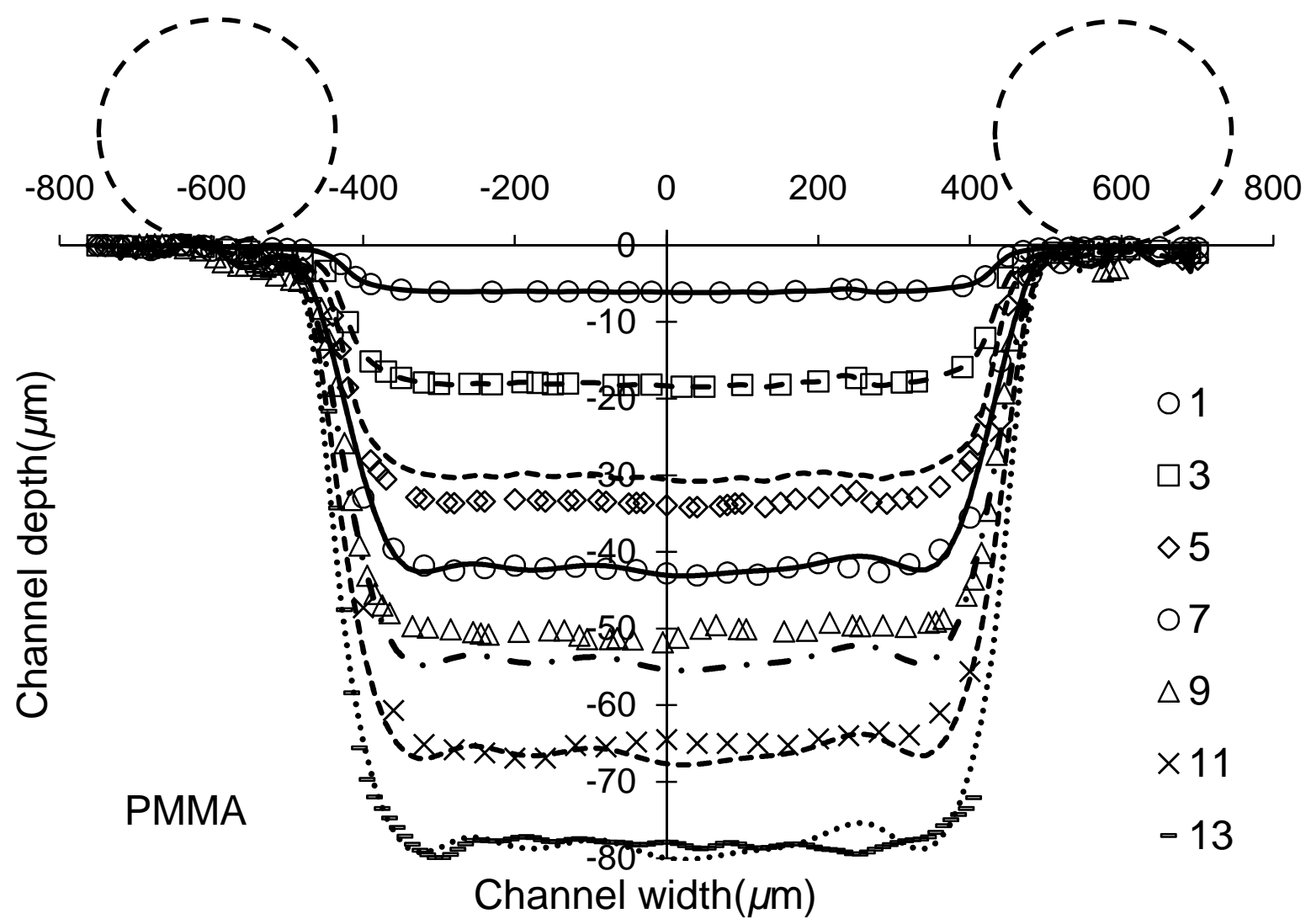

(c) 


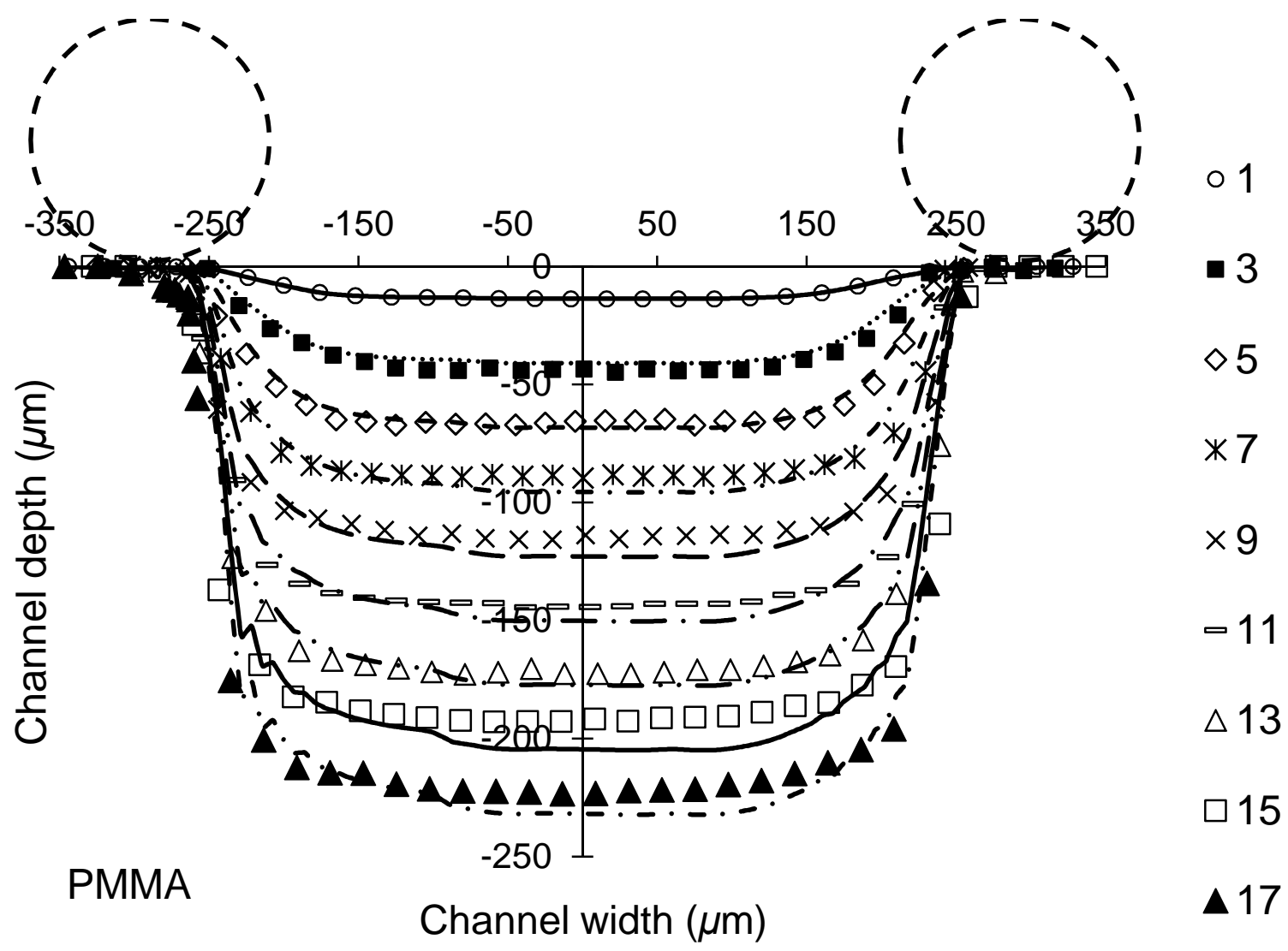

(d)

Fig. 5.9 Measured (symbols) and predicted (dashed lines) cross-sectional profiles of masked helical channels machined in a) rotating $5 \mathrm{~mm}$ glass rod, spring pitch $900 \mu \mathrm{m}$ (low aspect ratio), b) rotating $5 \mathrm{~mm}$ glass rod, spring pitch $700 \mu \mathrm{m}$ (high aspect ratio) c) rotating $4.72 \mathrm{~mm}$ PMMA rod, spring pitch $1200 \mu \mathrm{m}$ (low aspect ratio), d) rotating $4.72 \mathrm{~mm}$ PMMA rod, spring pitch 600 $\mu \mathrm{m}$ (high aspect ratio). Predictions used the model of Eq. (5.5). The maximum standard deviation of 3 measurements (explained in Section 5.2.4) at the channel center line depth was 8 $\mu \mathrm{m}, 20.25 \mu \mathrm{m}, 5 \mu \mathrm{m}$ and $9 \mu \mathrm{m}$ for parts a), b), c) and d) respectively. A dashed outline of spring (mask) location is shown in all the images. 


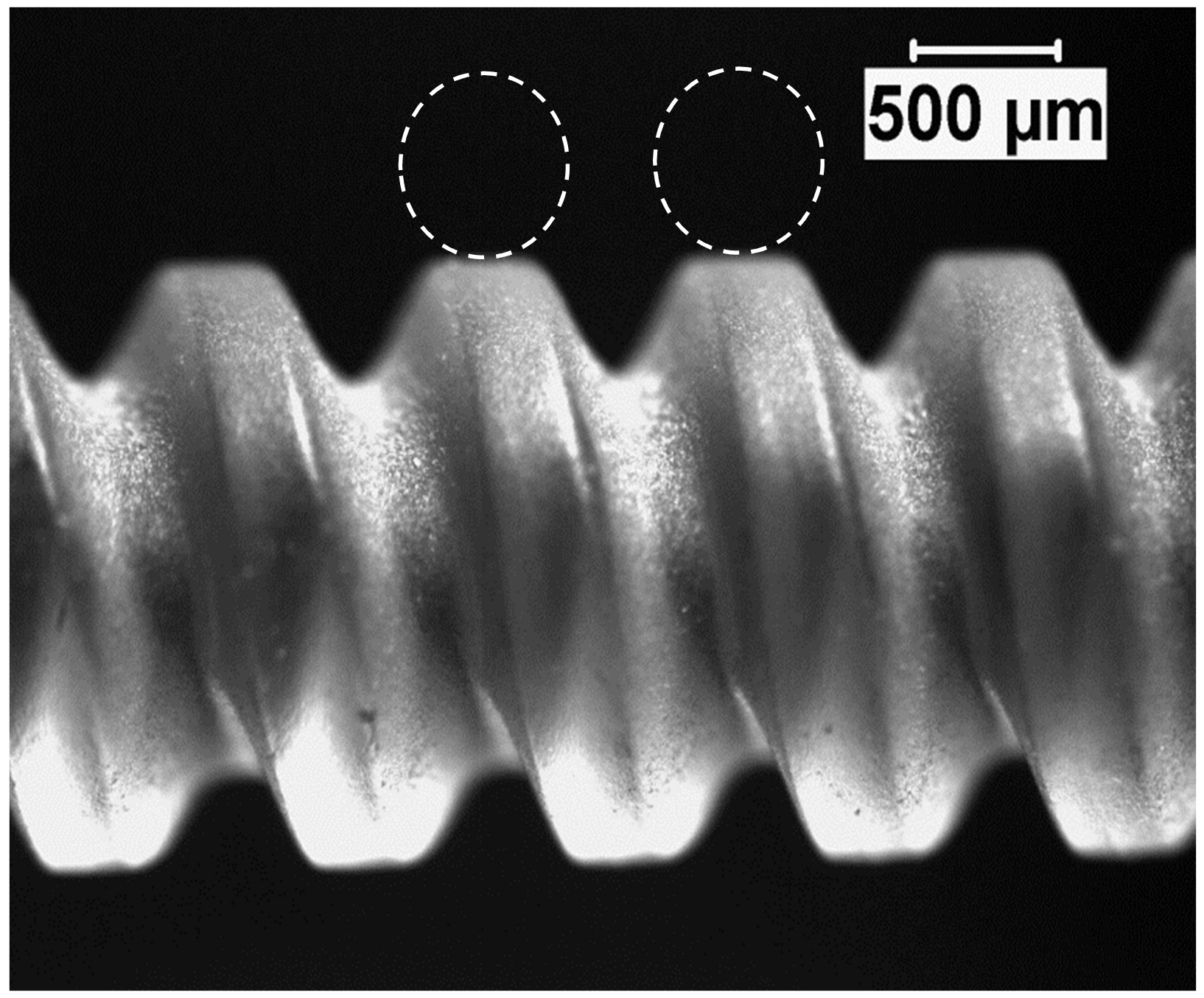

(a) 


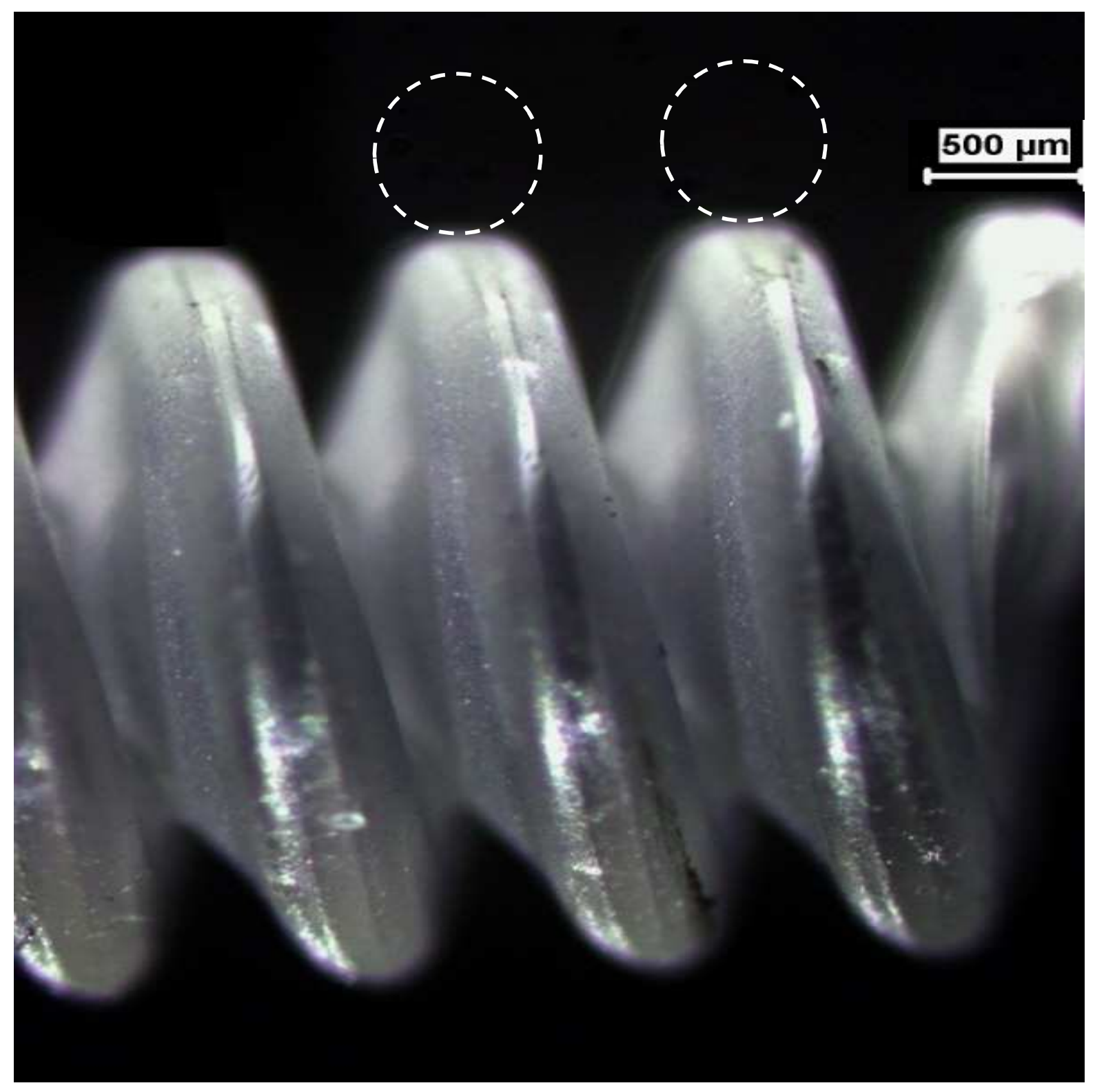

(b) 


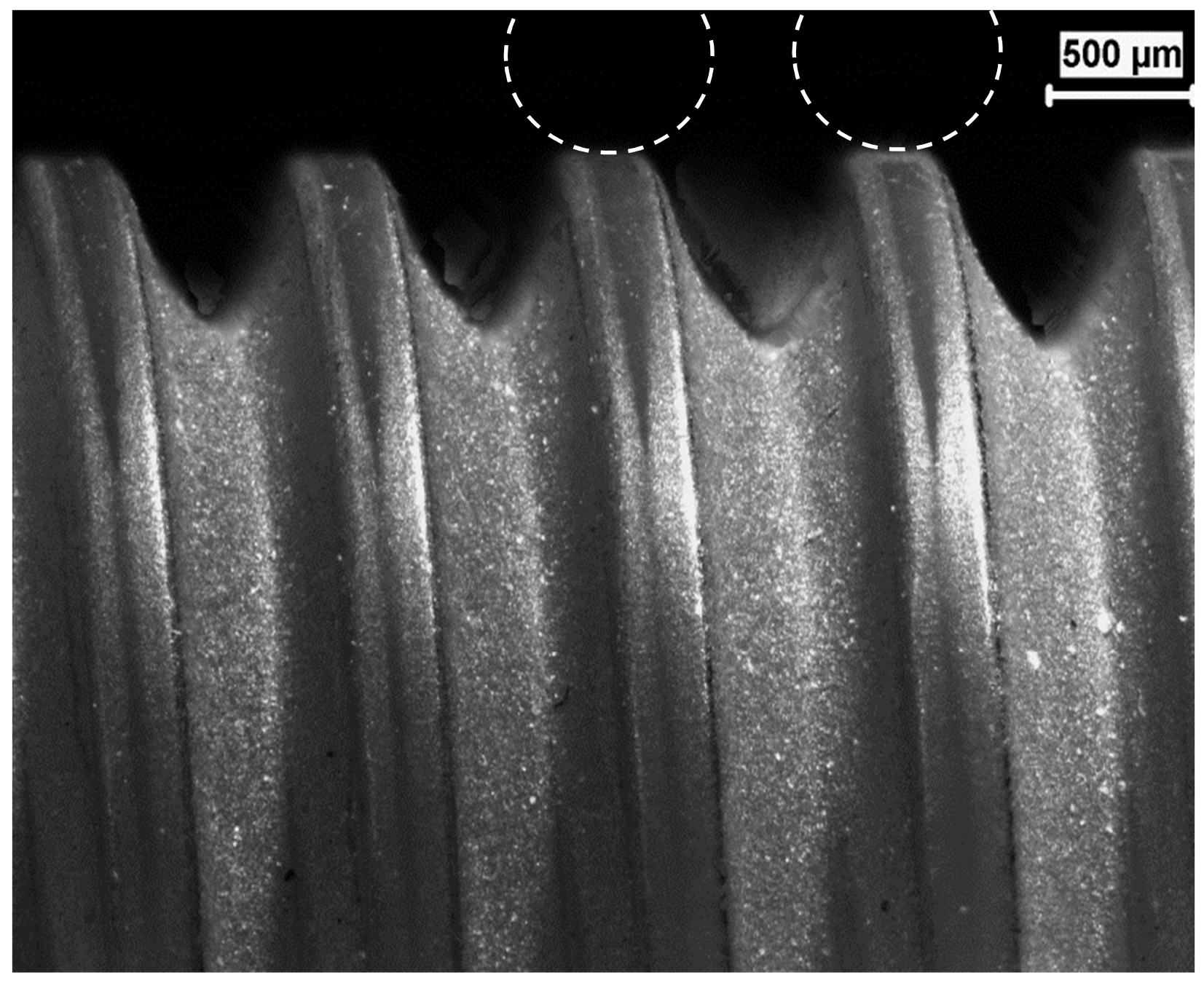

(c) 


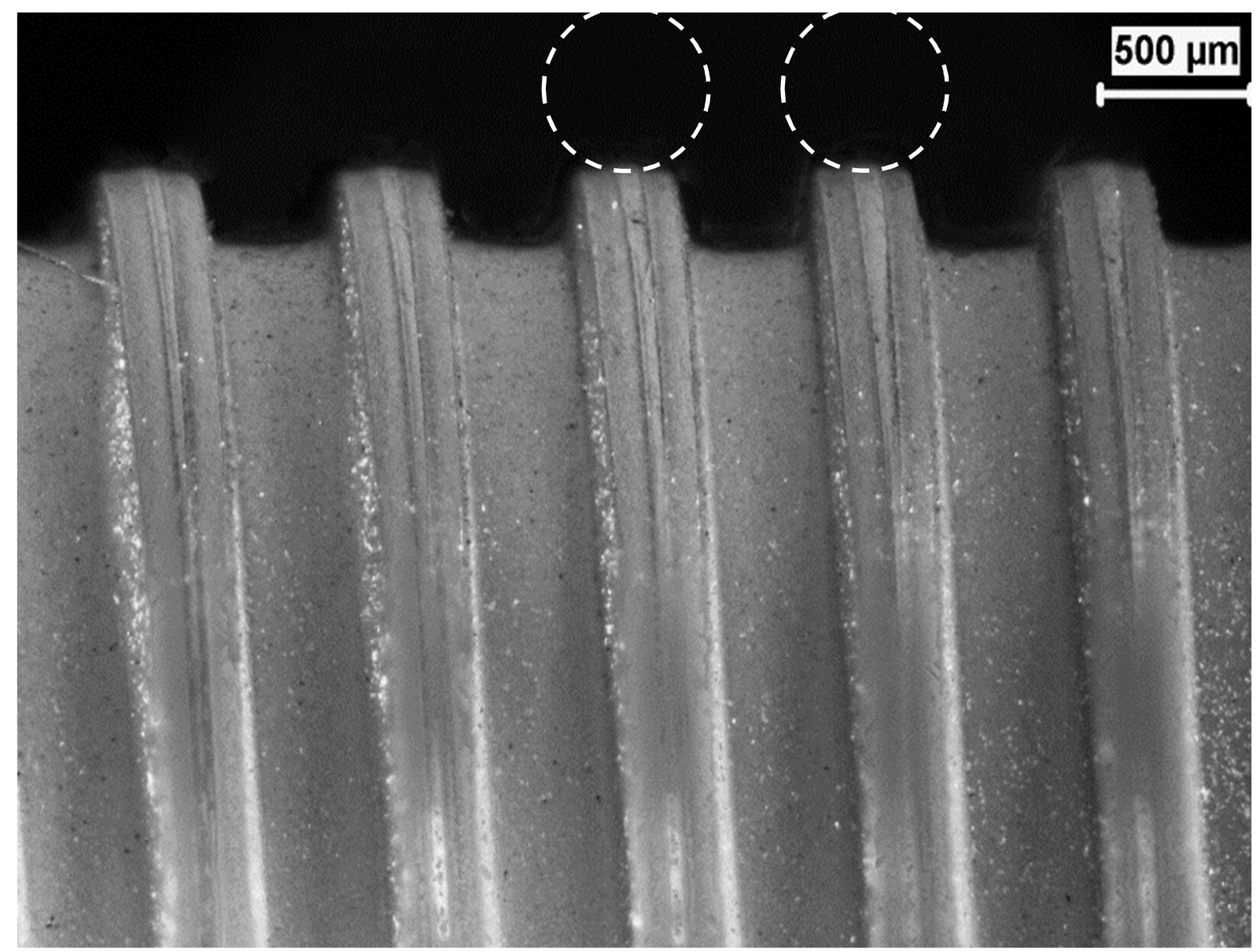

(d)

Fig. 5.10 Optical microscope images of helical micro-channels machined in: (a) $2 \mathrm{~mm}$ glass rod (aspect ratio 0.7, $500 \mu \mathrm{m}$ wire diameter, spring pitch $900 \mu \mathrm{m}$ ), (b) $3 \mathrm{~mm}$ glass rod (aspect ratio 1.2, $400 \mu \mathrm{m}$ wire diameter, spring pitch $800 \mu \mathrm{m}$ ), (c) $5 \mathrm{~mm}$ glass rod (aspect ratio 1, $700 \mu \mathrm{m}$ wire diameter, spring pitch $750 \mu \mathrm{m}$ ), and (d) $4.72 \mathrm{~mm}$ (aspect ratio 0.6, $400 \mu \mathrm{m}$ wire diameter, spring pitch $800 \mu \mathrm{m}$ ) PMMA rod. A dashed outline of spring (mask) location is shown in all the images. 


\subsubsection{Effect of mask spring wire diameter (mask thickness) on dimensions of helical channels}

The effect of the spring wire diameter (mask thickness) on the channel profiles was investigated by machining helical channels on a $5 \mathrm{~mm}$ diameter glass rod using $400 \mu \mathrm{m}$ and 750 $\mu \mathrm{m}$ diameter wires with an opening of $250 \mu \mathrm{m}$ between the edges of the adjacent coils. The experimental conditions were as in Section 2.4. Each channel was made at three depths using 3, 5 and 15 passes of the jet. It was observed that using the thinner spring produced statistically significant increases in the channel depth of 16\%,20\% and 30\% for the particle doses applied in 3,5 and 15 nozzle passes, respectively ( $t$-test, $p<0.05$ ). This trend is well known in AJM [83] and is attributed to an increase in the particle flux capable of erosion due to differences in particle ricochet from the mask edges, as shown in Fig. 5.11. For the case of thicker wire, particles which strike the edge of the mask will more likely rebound onto the opposite mask and lose their kinetic energy. With the thinner wire, more of the incident particles rebound directly to the target surface resulting in greater erosion (Fig. 5.11). Although the depth of the machined helical channels depended on the wire thickness for a given applied dose, the channel width was not affected significantly by the wire thickness, $(t$-test, $p>0.05)$ indicating that the thicker wire did not increase undercutting by particles striking beneath the wire (Fig. 5.12). These results indicate that the shallow-pass profile used to obtain the erosive efficacy distribution over the jet footprint ( $\delta$ and $\beta$ in Eqs. (5.9) and (5.10)) should be measured using the spring of the selected diameter.

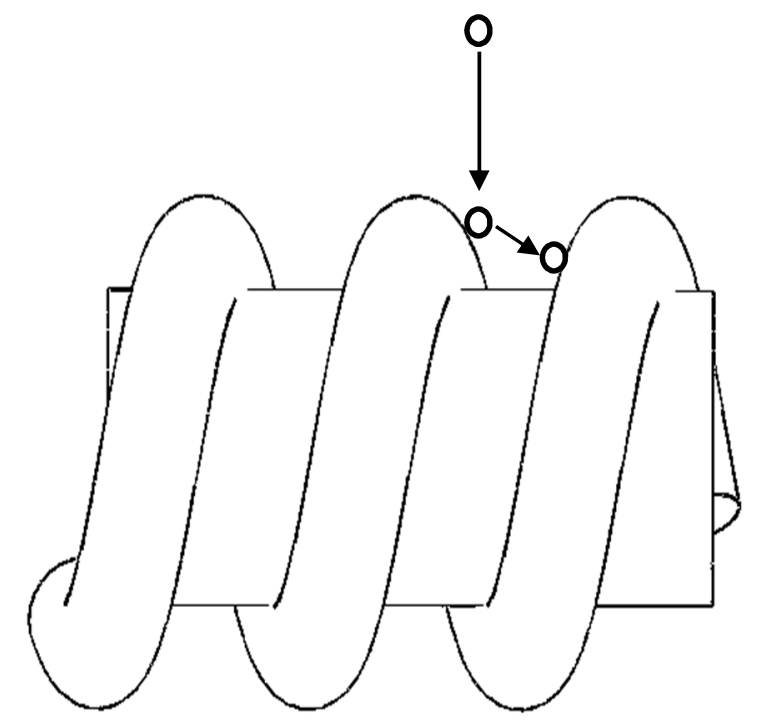

a

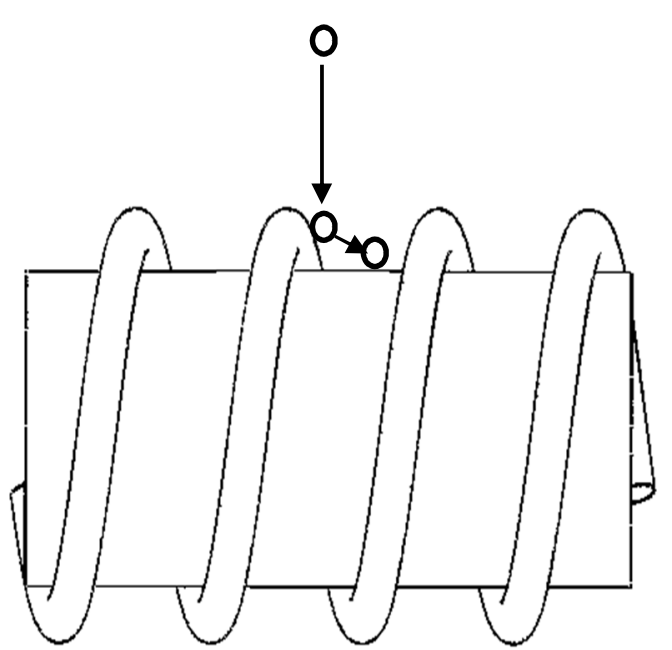

b 
Fig. 5.11 Particles striking the wire of a thick spring (a) were more likely to ricochet and strike the opposite coil, while those striking a thinner spring (b) had a higher probability of rebounding toward the surface of the rod.

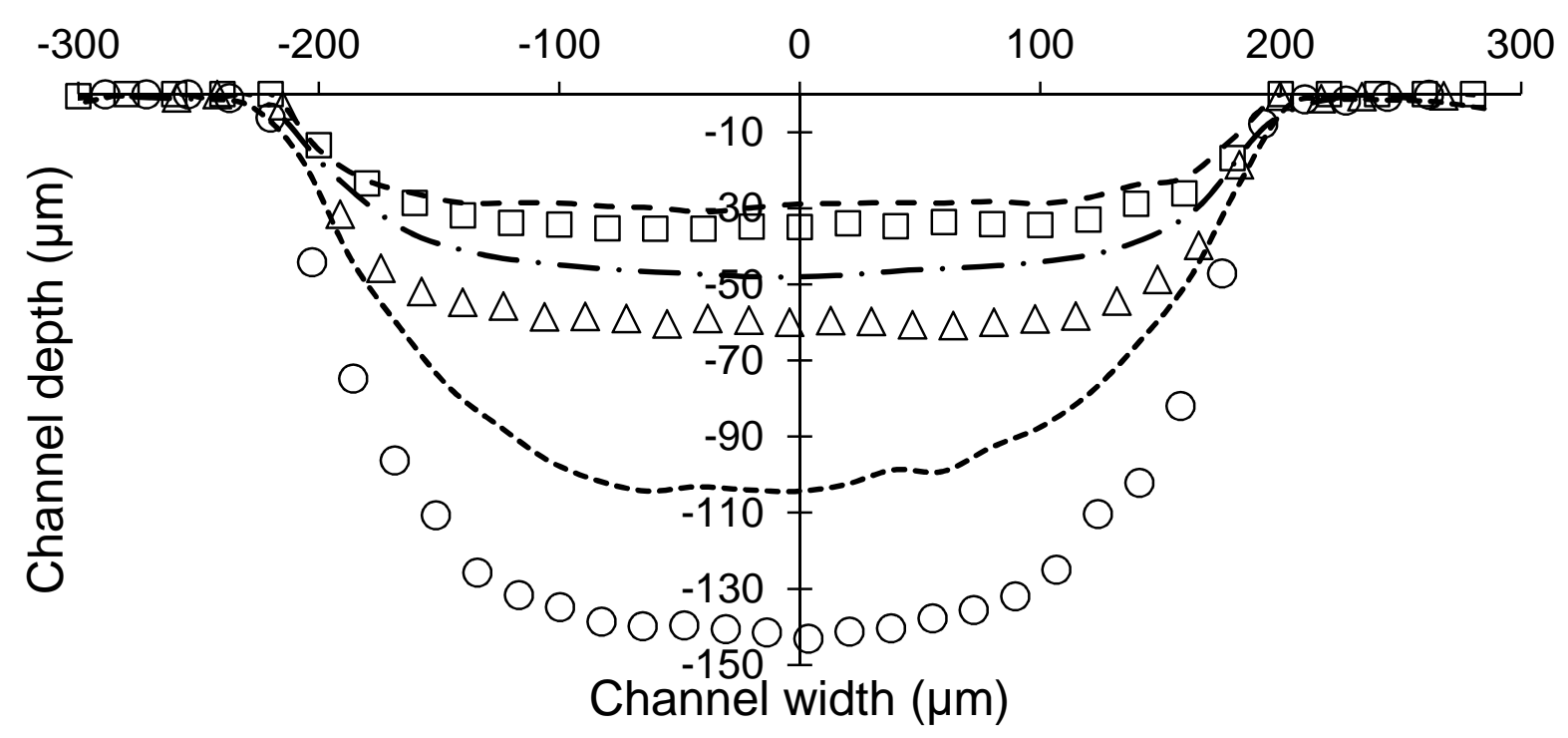

Fig. 5.12 Channel profiles machined on $5 \mathrm{~mm}$ diameter glass rod using a thin, $400 \mu \mathrm{m}$, (symbols) and a thick, $750 \mu \mathrm{m}$, (dashed lines) spring mask (The experimental conditions were as in Section 5.2.4). 


\subsubsection{Effect of spring pitch on the blast lag}

The mask opening width affects the inclination of channel sidewalls during the abrasive micro-machining, leading to 'blast lag' effect [13] in which wider channels machined in brittle materials are deeper than narrower channels for the same abrasive dose. For a smaller mask opening (or in this case smaller pitch), a V-shaped channel a develops at an earlier stage than a larger opening. Since the erosion of a brittle material is smaller on a sloped sidewall than a flat bottom, this results in a lower erosion rate for narrower channels [13]. In addition, the width at the bottom of the $\mathrm{V}$ shaped channel may become so narrow as to block the larger abrasive particles from eroding the channel centerline. For larger particles, the blast lag is more pronounced [13], thus in order to confirm that blast lag would exist for helical channels, larger $25 \mu \mathrm{m} \mathrm{Al}_{2} \mathrm{O}_{3}$ media was used to machine helical channels with different pitches. Two springs of same coil diameter, $300 \mu \mathrm{m}$, placed $15 \mathrm{~mm}$ apart, one with pitch $300 \mu \mathrm{m}$ and the other $650 \mu \mathrm{m}$, were used as masks on the same rod ( $5 \mathrm{~mm}$ diameter), so that the experiments could be carried out without turning off the micro-blaster to ensure that the mass flow rate remained unchanged. The experiments were repeated twice, each using 11 nozzle passes. As shown in Fig. 5.13, increasing the pitch size resulted in a $30 \%$ average increase in the channel depth.

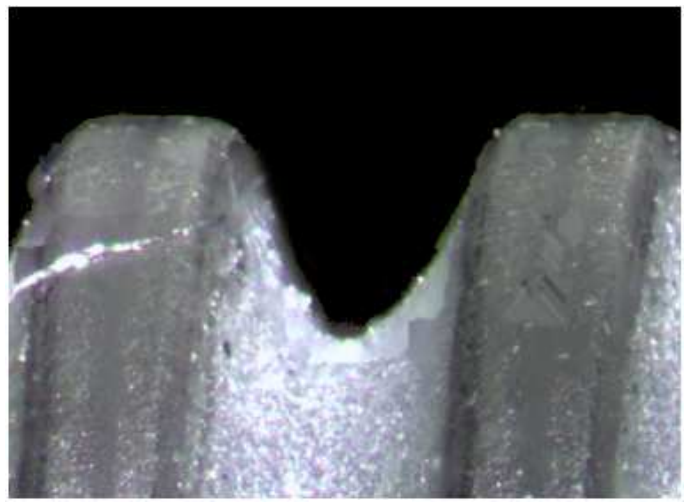

(a)

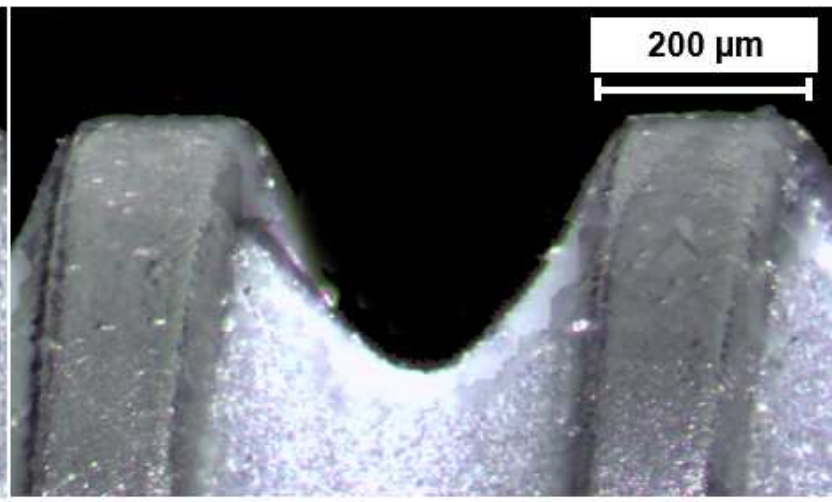

(b)

Fig. 5.13 The blast lag in a $5 \mathrm{~mm}$ glass rod illustrated for two channel widths. Wider channels made with a larger pitch $(650 \mu \mathrm{m}$ in (b)), became deeper for a given particle dose than narrower ones made using a smaller pitch, $(300 \mu \mathrm{m}$ in (a)). 


\subsection{Summary}

Abrasive air jet micro-machining (AJM) was investigated for use as an unmasked lathe operation, and as a means of machining masked helical patterns into rotating rods. A model was presented for the prediction of the volume of material removed in unmasked lathe operations. The model predictions were in good agreement with the measurements, showing a maximum 9\% error. The effect of nozzle offset was investigated for unmasked machining and it was found that the material removal increased by laterally offsetting the nozzle from the rod axis. Helical microchannels were machined in glass and PMMA rods of different radii using springs as masks. It was shown that the presented machining procedure was capable of fabricating a variety of low and high aspect ratio helical micro-channels. The surface evolution model of [11] was implemented for predicting the profiles of masked micro-channels machined on a $5 \mathrm{~mm}$ diameter glass rod and a $4.72 \mathrm{~mm}$ diameter PMMA rod. The predicted channel profiles matched the measured profiles quite well with maximum error of $7 \%$ in channel centerline depth. Using thicker spring wires as masks decreased the depth of machined channels, while it did not affect the channel width significantly. It was also found that increasing the spring pitch (mask opening) increased the depth of the channels for a given particle dose as a result of a reduction in the blast lag effect. The curvature of these helical channels creates the potential for useful applications in inertial microfluidic and biomedical devices for flow focusing and particle separation. 


\section{Chapter 6 Summary and Conclusions}

\subsection{Summary}

This dissertation was mainly focused on the abrasive micro-machining of stationary and rotating glass (brittle) and PMMA (ductile) highly curved substrates, i.e. rods. In Chapter 2 an unconvential technique was presented consisting of two steel strips attached to a nozzle for AJM of straight channels. The effects of nozzle standoff, shadow mask standoff, particle size and mask thickness on the frosted regions and the channel width were studied. A crossed arrangement of two mutually perpendicular sets of shadow masks was used to create a direct-writing device for generating arbitrarily curved paths. This apparatus was originally intended to be used as a masking methodology for machining micro- helical channels on rotating rods, but in order to avoid the complexity of synchronizing the rotational and axial feed speed of the lathe, it was not implemented. However, it still can to be utilized for future work if there is such a synchronization capability.

A modification to the existing surface evolution models for predicting channel profiles machined on brittle and ductile curved surfaces was presented in Chapter 3. In order to address all the shortcomings of existing models for the prediction of channel profiles machined on tilted and highly-curved surfaces, two major modifications were introduced; 1) the divergence angle of each particle trajectory in the jet plume and 2) change in local nozzle standoff were considered in the surface evolution model. The erosive efficacy on a flat surface at perpendicular incidence was measured using three nozzle standoff distances. The nozzle focus coefficient which was obtained from the bests fits to the dimensionless profiles of those three channels, was used in the modelling of channel profiles machined in tilted flat targets. However, $\beta$, nozzle focus coefficient, was modified for the case of curved surfaces. A computational fluid dynamic, CFD, modeling was implemented to justify this modification.

In Chapter 4, a computational fluid dynamics (CFD) procedure was presented for the prediction of the erosive footprint size in abrasive jet micro-machining (AJM) of both flat and curved targets. A double-pulsed laser shadowgraphy was implemented to measure the divergence of the abrasive particles and the axial and radial particle velocity components in the AJM jet. The divergence measurements were compared and verified by blasting holes through paper, as well. These results were used to simulate the AJM jet characteristics in the CFD modelling. The 
footprint measurements on flat target were performed at standoffs of 5, 10, 20, and $30 \mathrm{~mm}$ at a perpendicular incidence. The footprint measurements on curved surfaces was done on $3 \mathrm{~mm}$ and $5 \mathrm{~mm}$ glass rods. The results were compared and validated with the CFD simulations.

Chapter 5 investigated the use of air-driven abrasive jets as a lathe and as a means of machining helical patterns into rotating rods. A model was presented for the prediction of material removal during the machining of rotating and translating ductile (PMMA) and brittle (glass) rods using abrasive jet micro-machining (AJM). The experiments were conducted for 8 different helix angles while the rotation speed was kept constant and axial feed speed varied to achieve the desired helix angles, i.e. $10^{\circ}$ to $80^{\circ}$. In addition, a new experimental procedure for machining helical channels in glass and PMMA rods of different radii was introduced using a cylindrical steel spring as a mask. By adjusting the helical spring pitch (distance between two consecutive spring coils) the width of the channels was controlled and the desired depth could be achieved by adjusting the machining time. The surface evolution model presented in Chapter 3, was used to predict the shape of helical micro-channels. The effect of nozzle offset on the erosion rate, the effect of spring pitch (mask opening) on the blast lag and the effect of spring wire diameter (mask thickness) on the width and depth of helical channels was investigated. 


\subsection{Conclusions}

The main conclusions of this dissertation are:

1. The shadow mask technique was shown to be a practical alternative to the use of traditional masking methodologies which required attachment of the mask to the target. Implementation of the shadow mask made it possible to direct-write microchannels that were approximately $10 \%$ wider than those machined using clamped masks up to an aspect ratio of approximately 0.5. Moreover, it was demonstrated that by using two sets of crossed shadow masks the apparatus could be implemented as a direct write device for machining curved micro-structures. Sookhak Lari et al. [86] presented a method for AJM machining 3D micro-structures with prescribed topographies utilizing such a shadow mask.

2. It was shown that the jet spread angle and the change in the local standoff distance across the channel width must be taken into account in order to predict the crosssectional shapes of grooves machined on curved and tilted targets. The model predictions compared well with measured surface profiles machined in flat glass and PMMA targets with maximum error of $8 \%$. For tilted flat surfaces, the intersection of the jet cone with the surface was demonstrated to be representative of the erosive efficacy, while for the case of curved surfaces, to focus coefficient, $\beta$ had to be decreased to effectively account for second particle strikes.

3. CFD simulations demonstrated that the superposition of a primary and secondary conical abrasive jet plumes that were reflecting the first and secondary strikes, respectively, could determine the net erosive efficacy footprint. The simulations showed that approximately $90 \%$ of the particles within the primary plume affected the footprint size while the rest did not result in significant erosion. The predicted kinetic energies of particles striking a second time were found to be large enough to erode glass targets. The erosive footprint was also shown to depend on target curvature because the local slope changed due to the curvature. A higher curvature of the target resulted in a wider net erosive footprint. In general, it was shown that $\beta$ for a curved target could be determined either by CFD modelling through erosion 
map measurements or by adjusting it in the surface evolution model to fit the measured shallow first-pass profile of a channel machined on the curved target.

4. For unmasked machining of rotating and translating PMMA and glass rods, the model predictions and experimental measurements of material removal for 8 different helix angles from $10^{\circ}$ to $80^{\circ}$, were in good agreement. The predicted values were on average $12 \%$ greater than those measured for the $5 \mathrm{~mm}$ glass rod, and $6.5 \%$ greater for the $4.72 \mathrm{~mm}$ PMMA rod. It was shown that the spring mask machining procedure could be implemented conveniently for fabrication of microhelical channels with a wide range of aspect ratios and curvatures. It thus has the potential to be used as a means of fabricating threads on brittle (glass) materials which are difficult to machine with other machining methods. Highly curved microchannels have recently drawn considerable attention in inertial microfluidic and biomedical applications such as flow focusing, flow mixing and particle separation from fluid [78]. For instance, detecting bacteria using size-based separation, isolation of red blood cells and circulating tumor cells are typical important applications which utilize spiral patterns of channels, [82]. The significant advantage of presented helical micro-channels over other curved ones, e.g. spiral channels, is the constant radius of curvature that facilitates controlling and predicting the flow behavior, [82]. 


\subsection{Contributions}

The novel contributions of this dissertation can be summarized as:

1. A novel shadow mask apparatus was developed and implemented for machining straight micro-channels using a parallel mask configuration and direct machining using crossed masks.

2. A new definition of channel width was introduced which facilitated distinguishing between edge of frosting and micro-channel width. This definition provided a consistent means to determine the effect of process parameters on the channel and frosted area widths.

3. For the first time, a surface evolution model was used to predict the channel profiles machined on highly curved glass (brittle) and PMMA (ductile) targets. This was carried out by proposing two major modifications to the existing models: 1) considering the change in the local nozzle standoff distance and 2) taking into account the divergence angle of each particle trajectory in the abrasive air jet plume. Without these modifications, previous models resulted in erroneous predictions that showed significant deviation from the measured channel profiles.

4. The effect of second strike of rebounding particles on the jet footprint size and accordingly the nozzle focus coefficient, $\beta$, on curved surfaces was investigated for the first time. It was proposed that for curved targets, $\beta$ in the erosive efficacy expression had to be modified, i.e. the higher the curvature the larger was $\beta$. Otherwise, the footprint of the abrasive jet on the target was under-predicted and consequently the surface evolution model would lead to inaccurate predictions.

5. It was shown that the effective AJM footprint is characterized by the superposition of two cone-shaped plumes for first and second strike erosion. This verified that $\beta$, has to be modified in the erosive efficacy expression to accurately reflect the second strike effect on the erosive footprint size due to target curvature.

6. For the first time, the use of air-driven abrasive jets as a lathe was presented. It was used as a means of machining procedure for fabricating helical patterns into rotating rods of various radii, using cylindrical steel springs as masks. Due to its simple 
implementation in creating helical micro-channels on brittle (glass) and ductile (PMMA), it was extremely promising for fabrication of microfluidic devices. 


\subsection{Recommendations for Future work}

The research presented in this dissertation has the potential to be extended in the following aspects:

I. The shadow mask design can be improved by enabling the apparatus to hold the nozzle at oblique angles of attack. This would make it possible to machine asymmetric channels and thus broaden the variety of machinable features. In addition, the design should be modified to have more precise control on the mask opening.

II. Optimized nozzle standoff distance, particle size and mask thickness can be proposed to achieve the narrowest width for both straight and curved features.

III. The machining of groove on stationary rods in Chapter 3, can be extended to abrasive water jet and slurry jet machining, especially if metallic rods are to be machined. Due to complex nature of fluid flow CFD simulations combined with the modeling methods developed in this dissertation may be necessary.

IV. The proposed model of Chapter 3 can be implemented to targets of variable curvature rather than constant curvature (rods). This would allow predicting the shape of microfeatures which are machined on variety of curved targets.

V. The footprint size prediction and measurement of Chapter 4 may be also extended to substrates of variable curvature and its effect on the effective nozzle focus coefficient, $\beta$, could be investigated. This is an important step towards obtaining proper erosive efficacy function and accurate jet footprint size prediction on variable curvature workpieces.

VI. The lathe design presented in Chapter 5 can be improved by adding a controller to synchronize the rotational and axial feed speed; then the shadow mask apparatus of Chapter 2 can be utilized as mask for direct-write fabrication of helical micro-channels. 
VII. The helical micro-channels machining procedure presented in Chapter 5 could be used to create an inertial microfluidic rod-on-a-chip device facilitating the focusing of particles within a fluid. Such a chip would have to be sealed and parted. One possible approach is to cover the outer surface of the rod on which the helical channels are machined with a sheath made of PDMS, and injecting the fluid/particle mix of interest from one end of the rod to the helical channel. Then, the efficiency of the particle focusing can be evaluated considering the channel parameters, i.e. width, depth, curvature of the rod, helix angle, length of channel, etc.

VIII. Abrasive water jet and slurry jet machining can be used for fabrication of masked or unmasked helical micro-channels on metallic rods such as steel or copper. If performed masked, the mask material would have to be of a higher erosion resistance compared to the workpiece material. This may also require CFD simulations due to the complexity of liquid flow in water jet and slurry jet machining. 


\section{References}

[1] K. Furutani and H. Maeda, "Machining a glass rod with a lathe-type electro-chemical discharge machine," J. Micromechanics, vol. 18 pp. 261-269, 2008.

[2] I. Finnie, Erosion: Prevention and Useful Applications. ASTM International, vol. 664, pp. 36-58, 1979.

[3] I. Sheldon, G. L., Finnie, "The mechanisms of material removal in the erosive cutting of brittle materials," Journal of Engineering for Industry ASME, vol.88, pp. 393-400, 1996.

[4] J. Bitter, “A study of erosion phenomena part I,” Wear, vol. 6, pp. 5-21, 1963.

[5] J. G. A. Bitter, “A study of erosion phenomena part II,” Wear, vol. 6, no. 3, pp. 169-190, 1963.

[6] H. Getu, A. Ghobeity, J. K. Spelt, and M. Papini, "Abrasive jet micromachining of polymethylmethacrylate," Wear, vol. 263, pp. 1008-1015, 2007.

[7] D. S. Park, M.-W. Cho, and H. Lee, "Effects of the impact angle variations on the erosion rate of glass in powder blasting process," J. Advanced Manufacturing Technology, vol. 23, no. 5-6, pp. 444-450, 2004.

[8] J. Zahavi and G. F. Schmitt, "Solid particle erosion of reinforced composite materials," Wear, vol. 71, no. 2, pp. 179-190, 1981.

[9] A. Ghobeity, T. Krajac, T. Burzynski, M. Papini, and J. K. Spelt, "Surface evolution models in abrasive jet micromachining," Wear, vol. 264, pp. 185-198, 2008.

[10] H. Uuemis and I. Kleis, "A critical analysis of erosion problems which have been little studied," Wear, vol. 31, no. 2, pp. 359-371, 1975.

[11] P. Slikkerveer, P. C. . Bouten, and F. C. . de Haas, "High quality mechanical etching of brittle materials by powder blasting," Sensors Actuators A Phys., vol. 85, no. 1, pp. 296303, 2000.

[12] Y. Oka, H. Ohnogi, T. Hosokawa, and M. Matsumura, "The impact angle dependence of erosion damage caused by solid particle impact," Wear, vol. 203-204, pp. 573-579, 1997.

[13] H. Wensink and M. C. Elwenspoek, "Reduction of sidewall inclination and blast lag of powder blasted channels," Sensors Actuators A Phys., vol. 102, no. 1, pp. 157-164, 2002. 
[14] A. Pawlowski, A. Sayah, and M. Gijs, "Accurate masking technology for high-resolution powder blasting," J. Micromechanics and Microengineering, vol. 15, pp. 60-67, 2005.

[15] A. Sayah, V. K. Parashar, A. G. Pawlowski, and M. A. M. Gijs, "Elastomer mask for powder blasting microfabrication," Sensors Actuators A Phys., vol. 125, no. 1, pp. 84-90, 2005.

[16] H. Yagyu, S. Hayashi, and O. Tabata, "Application of nanoparticles dispersed polymer to micropowder blasting mask," J. Microelectromechanical Systems., vol. 13, no. 1, pp. 1-6, 2004.

[17] B. Karpuschewski, A. M. Hoogstrate, and M. Achtsnick, "Simulation and improvement of the micro abrasive blasting process," CIRP Ann. Manuf. Technol., vol. 53, no. 1, pp. 251254, 2004.

[18] F. Ahmadzadeh, S. Tsai, and M. Papini, "Effect of curing parameters and configuration on the efficacy of ultraviolet light curing self-adhesive masks used for abrasive jet micromachining," Precision. Engineering., vol. 49, pp. 354-364, 2017.

[19] A. Ghobeity, H. J. Crabtree, M. Papini, and J. K. Spelt, "Characterisation and comparison of microfluidic chips formed using abrasive jet micromachining and wet etching," $J$. Micromechanics and Microengineering, vol. 22, no. 2, p. 25014, 2012.

[20] H. Wensink, H. Jansen, and J. Berenschot, "Mask materials for powder blasting," $J$. Micomechanics and Microengineering, vol. 10, pp. 175-180, 2000.

[21] J. ten T. Boonkkamp and J. Jansen, "An analytical solution for mechanical etching of glass by powder blasting,” J. Engineering Mathematics., pp. 385-399, 2002.

[22] P. Slikkerveer, "Model for patterned erosion,” Wear, vol. 233-235, pp. 377-386, 1999.

[23] M. Verspui, G. de With, A. Corbijn, and P. Slikkerveer, "Simulation model for the erosion of brittle materials," Wear, vol. 233, pp. 436-443, 1999.

[24] Z. Moktadir and M. Kraft, "Analytical model of micromachining of brittle materials with sharp particles," J. Microelectronics, 36, (3-6), pp. 608-611, 2005.

[25] H. Wensink, J. W. Berenschot, H. V. Jansen, and M. C. Elwenspoek, "High resolution powder blast micromachining," in Proceedings IEEE Thirteenth Annual International Conference on Micro Electro Mechanical Systems (Cat. No.00CH36308), pp. 769-774, 2000. 
[26] A. Ghobeity, M. Papini, and J. K. Spelt, "Computer simulation of particle interference in abrasive jet micromachining," Wear, vol. 263, no. 1-6, pp. 265-269, 2007.

[27] R. Balasubramaniam, J. Krishnan, and N. Ramakrishnan, "A study on the shape of the surface generated by abrasive jet machining," J. Material Processesing Technology, vol. 121, no. 1, pp. 102-106, 2002.

[28] M. Achtsnick, P. F. Geelhoed, A. M. Hoogstrate, and B. Karpuschewski, "Modelling and evaluation of the micro abrasive blasting process," Wear, vol. 259, no. 1, pp. 84-94, 2005.

[29] A. Ghobeity, D. Ciampini, and M. Papini, "An analytical model of the effect of particle size distribution on the surface profile evolution in abrasive jet micromachining," J. Material Processesing Technology, vol. 209, pp. 6067-6077, 2009.

[30] S. Ally, J. K. Spelt, and M. Papini, "Prediction of machined surface evolution in the abrasive jet micro-machining of metals," wear, vol. 292-293, pp. 89-99, 2012.

[31] T. Burzynski and M. Papini, "Level set methods for the modelling of surface evolution in the abrasive jet micromachining of features used in MEMS and microfluidic devices," $J$. Micromechanics Microengineering, vol. 20, pp. 620-631, 2010.

[32] T. Matsumura and Y. Ueki, "Milling of micro grooves on glass cylinder surfaces," Int. J. Automation Technology, vol. 5, no. 1, pp. 11-20, Jan. 2011.

[33] W. Li, H. Zhu, J. Wang, Y. Ali, and C. Huang, "An investigation into the radial-mode abrasive waterjet turning process on high tensile steels," Int. J. Mechanical sciences, vol. 77, pp. 365-376, 2013.

[34] Z. Zhong and Z. Han, "Turning of glass with abrasive waterjet," J. Material and Manufacturing Processes, vol. 17, pp. 339-349, 2002.

[35] R. Manu and N. Babu, "An erosion-based model for abrasive waterjet turning of ductile materials," Wear, vol. 299, pp. 1091-1097, 2009.

[36] J. Cárach, S. Hloch, P. Hlaváček, and J. Ščučka, "Tangential turning of Incoloy alloy 925 using abrasive water jet technology," Int. J. of Advanced Manufacturing Technology, vol. 82, pp. 1747-1752, 2016.

[37] E. Belloy, A. Sayah, and M. Gijs, "Micromachining of glass inertial sensors," J. microelectromechanical system, vol. 11, pp. 85-90, 2002. 
[38] S. Schlautmann, H. Wensink, and R. Schasfoort, "Powder-blasting technology as an alternative tool for microfabrication of capillary electrophoresis chips with integrated conductivity sensors," J. Micomechanics Microengineering,vol. 11, pp. 386-389, 2001.

[39] D. S. Park, T. I. Seo, and M. W. Cho, "Mechanical etching of micro-pockets by powder blasting," Int. J. Advanced Manufacturing Technology, vol. 25, no. 11-12, pp. 1098-1104, Jun. 2005.

[40] C. Yamahata, F. Lacharme, Y. Burri, and M. Gijs, "A ball valve micropump in glass fabricated by powder blasting," Sensors Actuators B, vol. 110, pp. 1-7, 2005.

[41] M. Sugimoto, T. Shakouchi, and K. Hayakawa, "Gas-particle two-phase jet flow from slot nozzle and micro-blasting process," J. Ser. B Fluids, vol. 49, pp. 705-713, 2006.

[42] T. Lomas, A. Wisitsoraat, and F. Chevasuvit, "A precision hot embossing mold fabricated by high-resolution powder blasting with polydimethylsiloxane and SU-8 masking technology," J. Micomechanics Microengineering,vol. 19, pp. 480-489, 2009.

[43] Y. Liao and L. Chen, "A method of etching and powder blasting for microholes on brittle materials," J. Materials Processesing Technology, vol. 209, pp. 4390-4394, 2009.

[44] A. Ghobeity, H. Crabtree, M. Papini and J. K. Spelt "Characterisation and comparison of microfluidic chips formed using abrasive jet micromachining and wet etching," $J$. Micomechanics Microengineering, vol. 22, pp. 81-91, 2012.

[45] A. Ghobeity, H. Getu, T. Krajac, and J. K. Spelt, "Process repeatability in abrasive jet micromachining," J. Materials Processesing Technology, vol. 90, pp. 51-60, 2007.

[46] P. Slikkerveer, P. Bouten, F. I. Veld, and H. Scholten, "Erosion and damage by sharp particles," Wear, vol. 217, pp. 237-250, 1998.

[47] D. Marshall and B. Lawn, "Elastic/plastic indentation damage in ceramics: the lateral crack system,” J. Am. Ceram. Soc., vol. 65, pp. 561-566, 1982.

[48] H. Li, J. Wang, and J. Fan, "Analysis and modelling of particle velocities in micro-abrasive air jet," Int. J. Machine Tools and manufacture, vol. 49, pp. 850-858, 2009.

[49] M. Papini, D. Ciampini, T. Krajac, and J. K. Spelt, "Computer modelling of interference effects in erosion testing: effect of plume shape," Wear, vol. 255, pp. 85-97, 2003. 
[50] A. Martinez and E. Chaikof, "Microfabrication and nanotechnology in stent design," Wiley Interdiscip. Rev., vol.3, pp. 256-268, 2011.

[51] A. Ghobeity, H. Getu, M. Papini and J. K. Spelt, "Surface evolution models for abrasive jet micromachining of holes in glass and polymethylmethacrylate (PMMA), "J. Micomechanics Microengineering, vol. 17, pp. 2175-2185, 2007.

[52] M. Kong, S. Anwar, J. Billingham, and D. Axinte, "Mathematical modelling of abrasive waterjet footprints for arbitrarily moving jets: part I-single straight paths," Int. J. Machine Tools and Manufacture, vol. 53, pp. 56-68, 2012.

[53] H. Kim, I. Lee, and T. Ko, "Direct 3D mask modeling for nonplanar workpieces in microabrasive jet machining," Int. J. Advanced Manufacturing Technology, vol. 58, pp 175-186, 2012.

[54] T. Burzynski and M. Papini, "Measurement of the particle spatial and velocity distributions in micro-abrasive jets," Measurment Science abd Technology, vol. 22, pp. 285-296, 2011.

[55] H. Li, A. Lee, J. Fan, G. Yeoh, and J. Wang, "On DEM-CFD study of the dynamic characteristics of high speed micro-abrasive air jet," Powder Technology, vol. 267, pp. 161179, 2014.

[56] D. Dehnadfar, J. Friedman, and M. Papini, "Laser shadowgraphy measurements of abrasive particle spatial, size and velocity distributions through micro-masks used in abrasive jet micro-machining," J. Material Processesing technology, vol. 212, pp. 137-149, 2012.

[57] P. Shipway and I. Hutchings, "Influence of nozzle roughness on conditions in a gas-blast erosion rig," Wear, vol.162, pp. 148-158, 1993.

[58] H. Nouraei, A. Wodoslawsky, M. Papini and J. K. Spelt, "Characteristics of abrasive slurry jet micro-machining: a comparison with abrasive air jet micro-machining," J. Material Processesing Technology, vol. 213, pp. 1711-1724, 2013.

[59] D. Solignac, A. Sayah, S. Constantin, and R. Freitag, "Powder blasting for the realisation of microchips for bio-analytic applications," Sensors actuators A, vol. 92, pp. 388-393, 2001. 
[60] C. Liu, J. Chen, J. Engel, J. Zou, X. Wang, and Z. Fan, "Polymer micromachining and applications in sensors, Microfluidics, and Nanotechnology, 226th National Meeting of the American Chemical Society" 2003.

[61] P. Shipway, "The effect of plume divergence on the spatial distribution and magnitude of wear in gas-blast erosion," Wear, vol. 205, pp. 169-177, 1997.

[62] A. Mansouri, M. Mahdavi, S. Shirazi, and B. McLaury, "Investigating the effect of sand concentration on erosion rate in slurry flows," Nace Internationl, 2015.

[63] H. Qi, D. Wen, Q. Yuan, L. Zhang, and Z. Chen, "Numerical investigation on particle impact erosion in ultrasonic-assisted abrasive slurry jet micro-machining of glasses," Powder Technology, vol. 314, pp. 627-634, 2016.

[64] H. Qi, D. Wen, C. Lu, and G. Li, "Numerical and experimental study on ultrasonic vibration-assisted micro-channelling of glasses using an abrasive slurry jet," Int. J. Mech. Sci., vol. 314, pp. 627-634, 2016.

[65] K. Kowsari, H. Nouraei, B. Samareh, M. Papini and J. K. Spelt, "CFD-aided prediction of the shape of abrasive slurry jet micro-machined channels in sintered ceramics," Ceramics, vol. 42, pp. 7030-7042, 2016.

[66] A. Nouhi, K. Kowsari, J. K. Spelt, and M. Papini, "Abrasive jet machining of channels on highly-curved glass and PMMA surfaces,” Wear, vol. 356-357, pp. 30-39, 2016.

[67] V. Hadavi, B. Michaelsen, and M. Papini, "Measurements and modeling of instantaneous particle orientation within abrasive air jets and implications for particle embedding," Wear, vol. 336-337, pp. 9-20, 2015.

[68] C. Crowe, J. Schwarzkopf, M. Sommerfeld, and Y. Tsuji, Multiphase flows with droplets and particles. CRC Press, 2011.

[69] K. Kowsari, M. R. Sookhaklari, H. Nouraei, M. Papini and J. K. Spelt, "Hybrid erosive jet micro-milling of sintered ceramic wafers with and without copper-filled through-holes," $J$. Material Processesing Technology, vol. 230, pp. 198-210, 2016.

[70] J. Humphrey, "Fundamentals of fluid motion in erosion by solid particle impact," Int. J. Heat Fluid Flow, vol. 11, pp. 170-195, 1990.

[71] A. Haider and O. Levenspiel, "Drag coefficient and terminal velocity of spherical and nonspherical particles," Powder Technology, vol. 58, pp. 6, 1989. 
[72] H. Wensink and M. Elwenspoek, "A closer look at the ductile-brittle transition in solid particle erosion," Wear, vol. 253, pp. 1035-1043, 2002.

[73] V. Hadavi, C. Moreno, and M. Papini, "Numerical and experimental analysis of particle fracture during solid particle erosion, Part II: Effect of incident angle, velocity and abrasive size," Wear, vol. 356-357, pp. 146-157, 2016.

[74] H. Getu, A. Ghobeity, J. K. Spelt, and M. Papini, "Abrasive jet micromachining of acrylic and polycarbonate polymers at oblique angles of attack," Wear, vol. 265, pp. 888-901, 2008.

[75] C. J. Morgan, R. R. Vallance, and E. R. Marsh, "Micro machining glass with polycrystalline diamond tools shaped by micro electro discharge machining," J. Micromechanics Microengineering, vol. 14, no. 12, pp. 1687-1692, 2004.

[76] H. Degawa, N. Urano, and S. Matsuo, "Laser Fabrication of Miniature Internal Thread in Glass Substrate," J. Micromachines Microengineering, vol. 8, no. 2, pp. 48-59, 2017.

[77] E. Sogabe, K. Ohashi, N. Lu, M. Fujiwara, T. Onishi, and S. Tsukamoto, "Machining characteristics of cylindrical blasting and application to micro patterning," Advanced Materials Research, vol. 325, pp. 570-575, Aug. 2011.

[78] D. Di Carlo, “Inertial microfluidics.," Lab Chip, vol. 9, no. 21, pp. 3038-3046, 2009.

[79] R. H. Liu, M. A. Stremler, K. V. Sharp, M. G. Olsen, J. G. Santiago, R. J. Adrian, H. Aref, and D. J. Beebe, "Passive mixing in a three-dimensional serpentine microchannel," $J$. Microelectromechanical Systems, vol. 9, no. 2, pp. 190-197, Jun. 2000.

[80] A. Sudarsan and V. Ugaz, "Multivortex micromixing," Proceedings of the National Academy of Sciences., vol. 103, pp. 7228-7233, 2006.

[81] R. Manlapaz and S. Churchill, "Fully developed laminar flow in a helically coiled tube of finite pitch," Chem. Eng., vol. 7, pp.57-78,1980.

[82] W. Lee, D. Kwon, W. Choi, G. Jung, A. Au, and A. Folch, “3D-printed microfluidic device for the detection of pathogenic bacteria using size-based separation in helical channel with trapezoid cross-section," Sci. Rep., vol. 5, pp. 345-353, 2015.

[83] A. Nouhi, M. Lari, J. Spelt, and M. Papini, "Implementation of a shadow mask for direct writing in abrasive jet micro-machining," J. Material Processesing Technology, vol. 223, pp. 232-239, 2015. 
[84] R. Haj Mohammad Jafar, J. K. Spelt, and M. Papini, "Surface roughness and erosion rate of abrasive jet micro-machined channels: Experiments and analytical model," Wear, vol. 303, no. 1, pp. 138-145, 2013.

[85] R. Haj Mohammad Jafar, M. Papini, and J. K. Spelt, "Simulation of erosive smoothing in the abrasive jet micro-machining of glass," J. Material Processesing Technology, vol. 213, no. 12, pp. 2254-2261, 2013.

[86] M. R. Sookhak Lari, M. Teti, and M. Papini, "Inverse methods to gradient etch threedimensional features with prescribed topographies using abrasive jet micro-machining: Part II-Verification with micro-machining experiments," Precision Engineering, vol. 45, pp. 262-271, Jul. 2016. 